NUREG/CR-6210

PNL-10496

\title{
Computer Codes for Evaluation of Control Room Habitability (HABIT)
}

Manuscript Completed: October 1995

Date Published: June 1996

Prepared by

S. A. Stage

Pacific Northwest Laboratory

Richland, WA 99352

J. Schaperow, NRC Project Manager

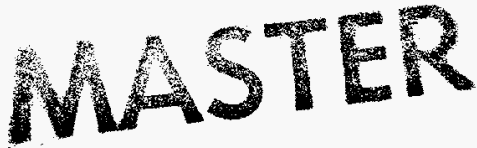

Prepared for

Division of Systems Technology

Office of Nuclear Regulatory Research

U.S. Nuclear Regulatory Commission

Washington, DC 20555-0001

NRC Job Code L2560

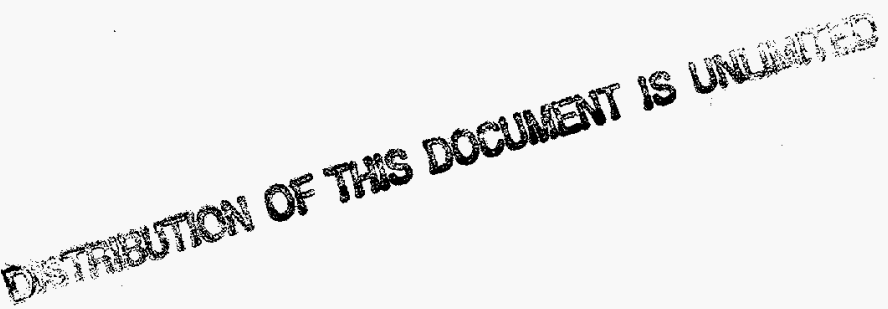




\section{DISCLAIMER}

This report was prepared as an account of work sponsored by an agency of the United States Government. Neither the United States Government nor any agency thereof, nor any of their employees, make any warranty, express or implied, or assumes any legal liability or responsibility for the accuracy, completeness, or usefulness of any information, apparatus, product, or process disclosed, or represents that its use would not infringe privately owned rights. Reference herein to any specific commercial product, process, or service by trade name, trademark, manufacturer, or otherwise does not necessarily constitute or imply its endorsement, recommendation, or favoring by the United States Government or any agency thereof. The views and opinions of authors expressed herein do not necessarily state or reflect those of the United States Government or any agency thereof. 


\section{DISCLAIMER}

Portions of this document may be illegible in electronic image products. Images are produced from the best available original document. 
HABIT Manual

NUREG / CR- 6210

ABSTRACT

This report describes The Computer Codes for Evaluation of Control Room Habitability (HABIT). HABIT is a package of computer codes designed to be used for the evaluation of control room habitability in the event of an accidental release of toxic chemicals or radioactive materials. Given information about the design of a nuclear power plant, a scenario for the release of toxic chemicals or radionuclides, and information about the air flows and protection systems of the control room, HABIT can be used to estimate the chemical exposure or radiological dose to control room personnel.

HABIT is an integrated package of several programs that previously needed to be run separately and required considerable user intervention. These are EXTRAN, CHEM, TACT5, FPFP_2, and CONHAB. New input routines have been written for these routines using data input windows. These are designed for easy use when entering, reviewing, and revising the data. The programs can now be run in sequence as an integrated package. Improvements have been made in the computational methods used by some of the routines. The programs produce files containing ASCII tables of values and output files that can readily be imported into a commercial spreadsheet to be graphed or for further computations.

This report discusses the theoretical basis and physical assumptions made by each of the modules in HABIT and gives detailed information about the data entry windows. Sample runs are given for each of the modules. A brief section of programing notes is included.

A set of computer disks will accompany this report if the report is ordered from the Energy Science and Technology software Center. The disks contain the files needed to run HABIT on a personal computer running DoS. Source codes for the various HABIT routines are on the disks. Also included are input and output files for three demonstration runs. 

LIST OF FIGURES ........................ ix

LIST OF TABLES........................ xii

EXECUTIVE SUMMARY $\ldots \ldots \ldots \ldots \ldots \ldots \ldots \ldots \ldots \ldots \ldots \ldots \ldots$

ACKNOWLEDGEMENTS ........................ xvi

1. AN OVERVIEW OF THE CONTROL ROOM HABITABILITY

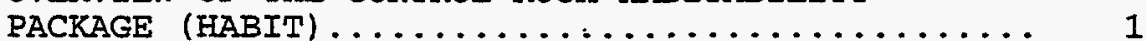

1.1. A Brief Description of HABIT............... 1

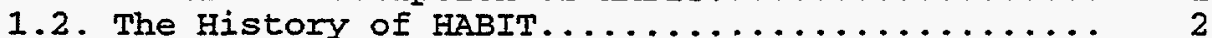

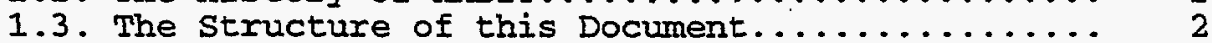

1.4. Before Starting the HABIT Programs............ 3

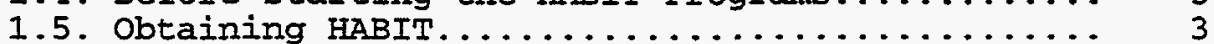

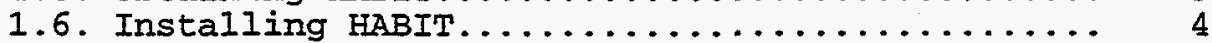

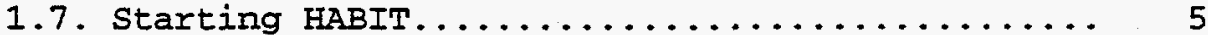

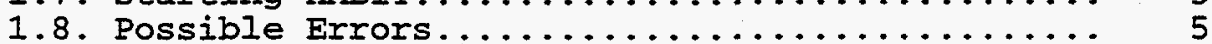

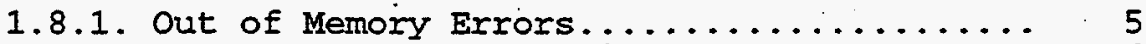

1.8.2. Incorrect output of Time steps............ 6

1.8.3. Out of Environment Space............... 6

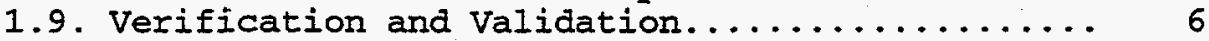

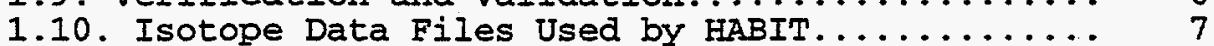

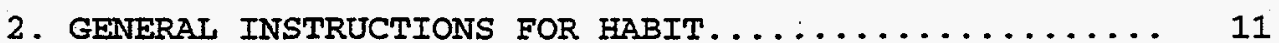

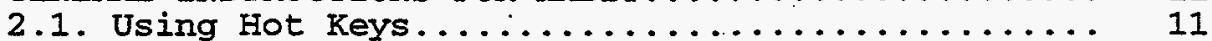

2.2. Using the Mouse....................... 12

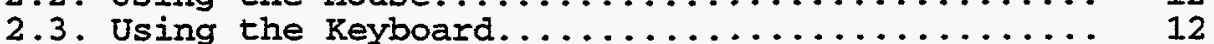

2.4. Use of the Escape Key, <Esc>............... 12

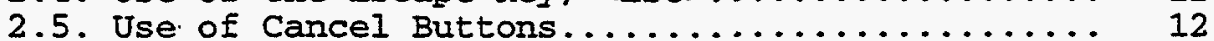

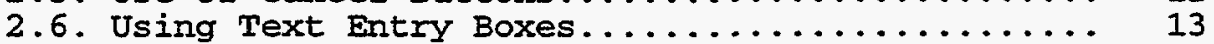

2.7. Using Value Entry Boxes.................. 13

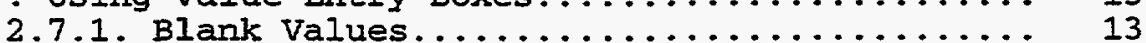

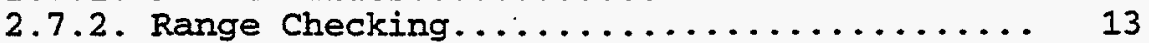

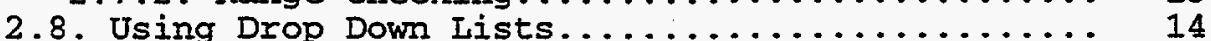

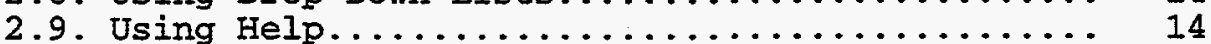

3. THE USE OF FILES BY HABIT.................. 15

3.1. HABIT File Naming Conventions............... 15

3.2. Using the File Name and File Path windows...... 17

4. THE HABIT MAIN WINDOW.................... 21

5. THE DESIGN EDIT WINDOW................... 25 
6. EXTRAN: A COMPUTER CODE FOR ESTIMATING CONCENTRATIONS OF TOXIC SUBSTANCES AT CONTROL ROOM AIR INTAKES..................... 6.1. General Description of EXTRAN............. 27

6.2 . The Technical Basis for EXTRAN............. 28

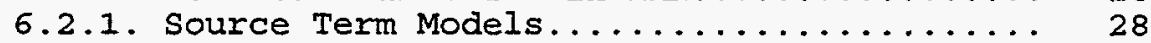
6.2 .1 .1 . Gas Tank Bursts and Leaks.......... 29 6.2.1.2. Liquid Tank Bursts and Leaks........ 29 6.2 .1 .2 .1 . Mass Balance for Liquefied

Gasses and Volatile Liquids.. 29

6.2 .1 .2 .1 .1 . Flashing............ 30

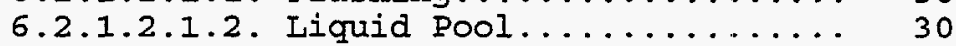

6.2 .1 .2 .1 .3 . Evaporation............. 31 6.2.1.2.2. Energy Balance for Liquefied Gasses and Volatile Liquids.. 32

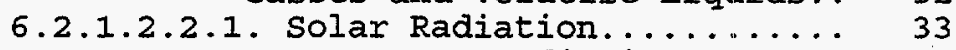

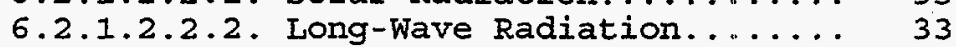

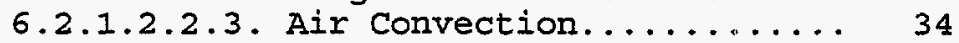

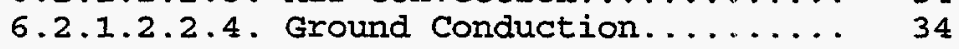

6.2.1.2.2.5. Latent Heat Transfer...... 35

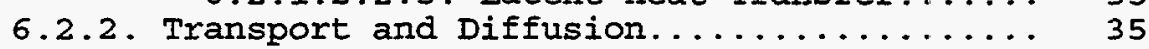

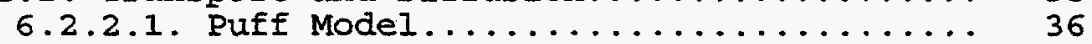

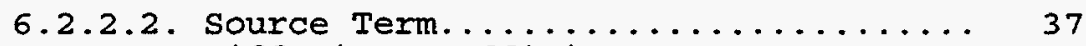

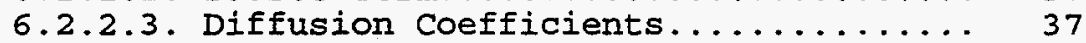

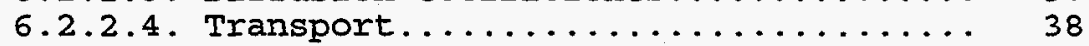

6.2 .3 . Computational Time step and Duration of

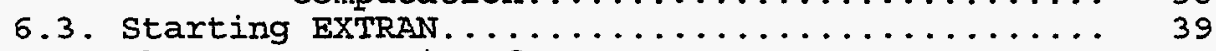

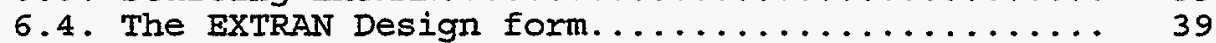

6.5 . Form for ExTRAN Data Entry............... 48

7. THE CHEM PROGRAM.................... 53

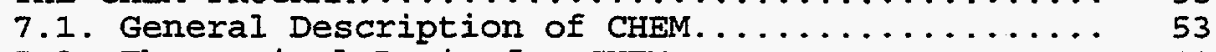

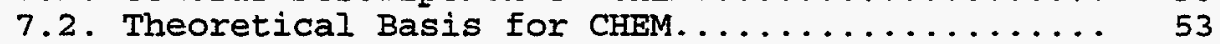

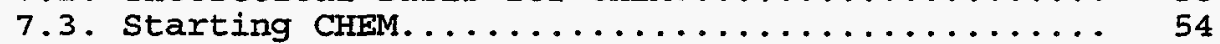

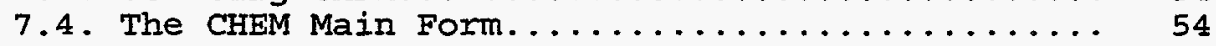

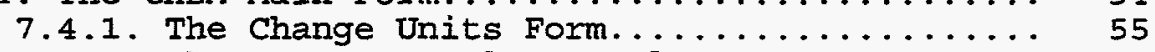

7.4.2. The CHEM Control Room Flow Parameters

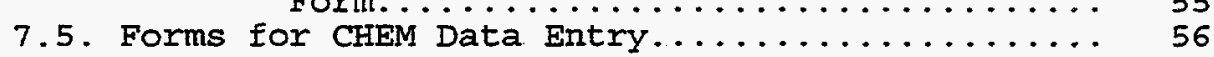

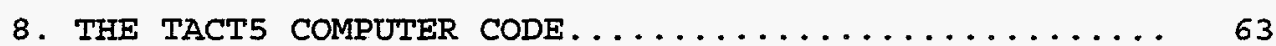

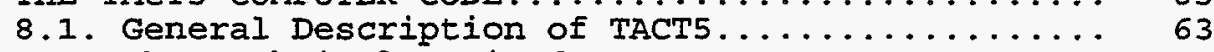

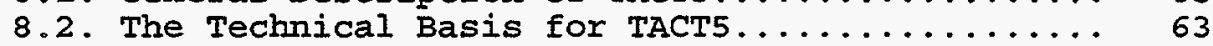

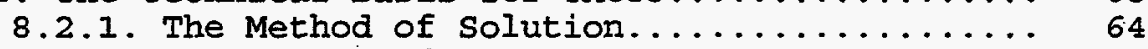

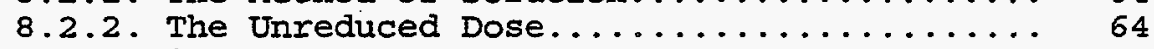

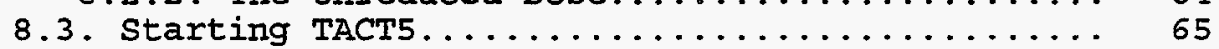

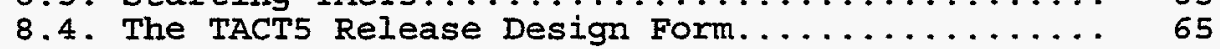

8.5. The TACT5 Nuclide Selection Form.............. 67

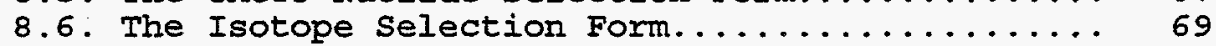

8.7. The TACT5 Node Design Form................ 69

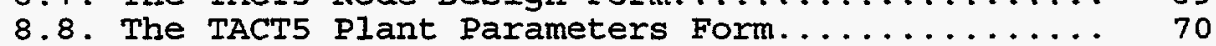

8.9. Entering Time Dependent Data............................ 70

8.9.1. The TACT5 Time Dependent Variables Form... 71 
8.9.2. The TACT5 Time Dependent Variable Input

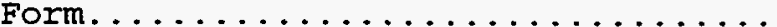

8.9.2.1. Information at the Top of the TACT5 Time Dependent Variable Input Form

8.9.2.2. The Data Table at the Middle of the TACT5 Time Dependent Variable

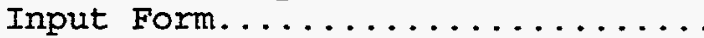

8.9.2.3. The Buttons at the Bottom of the TACT5 Time Dependent Variable

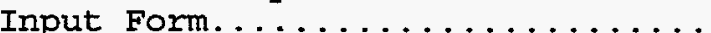

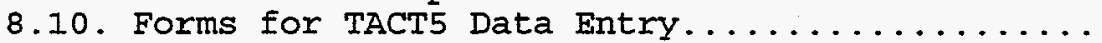

9. FPFP 2: A CODE FOR FOLLOWING AIRBORNE FISSION

PR̄ODUCTS IN GENERIC NUCLEAR PLANT FLOW PATHS.... 9.1. General Description of FPFP $2 \ldots \ldots \ldots \ldots \ldots \ldots$

9.2 . The Technical Basis for FPFP $2 \ldots \ldots \ldots \ldots \ldots \ldots$

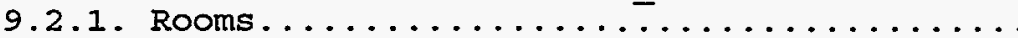

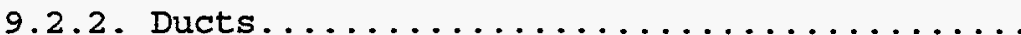

9.2.3. Filters.....................

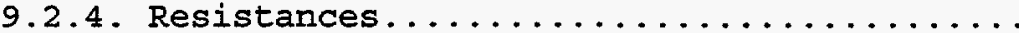

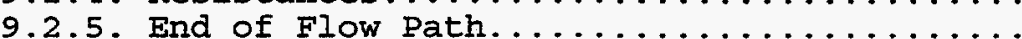
9.3. Fission Product Surface Deposition, Setting,

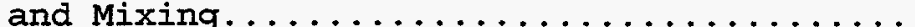

9.3.1. Surface Deposition and Mixing of Iodine

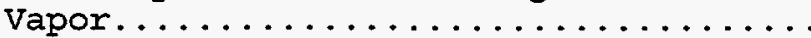

9.3.2. Settling and Spray Removal of Particles...

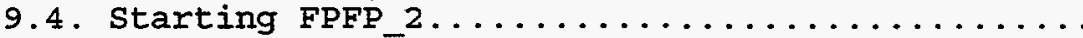

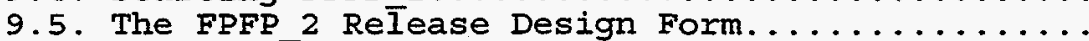

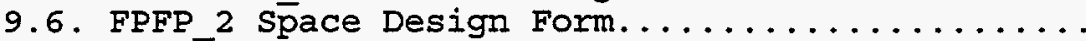

9.7. FPFP 2 Flow Design Form.................

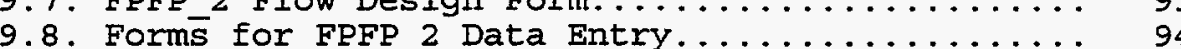

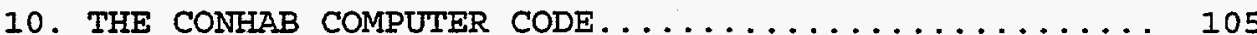

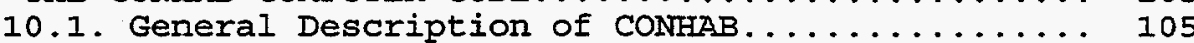

10.2. Theoretical Basis for CONHAB.............. 106

10.2.1. Treatment of Isotopes and Forms within

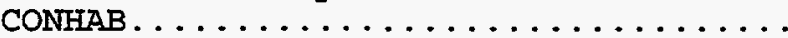

10.2.2. Treatment of Unfiltered Inflows from

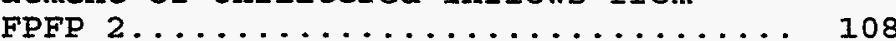

10.2.3. Treatment of Filtered Inflows from TACT5. 108

10.2.4. How Filters are Assumed to Work within

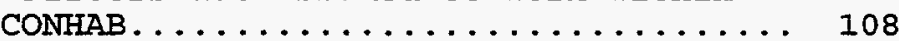

10.2.5. Atmospheric Diffusion used by CONHAB.... 109

10.3. CONHAB Compared with the Murphy-Campe Method.. 109

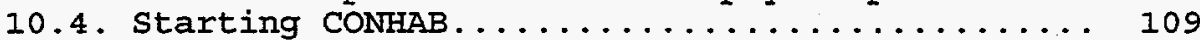

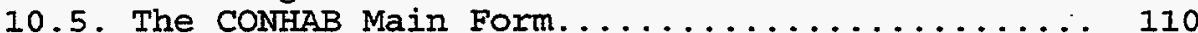

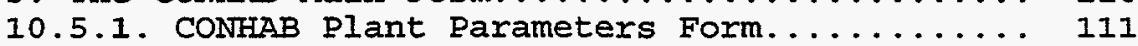

10.5.2. The Change Units Form................ 111

10.5.3. CONHAB Meteorological Parameters Form.................... 112

10.5.4. The CONHAB Control Room Flow Parameters

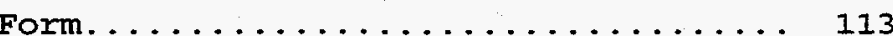

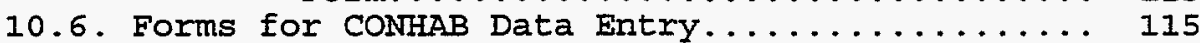




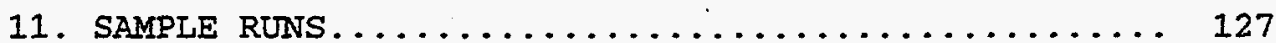

11.1. DEMO1, A Hypothetical Toxic Chemical Release.. 127

11.2. DEMO2, A Hypothetical Radioactive Release

Through TACT5 ................... 131

11.3. DEMO3, A Hypothetical Radioactive Release

Through FPFP_2 . . . . . . . . . . . 138

12. PROGRAMMING NOTES FOR HABIT ............. 141

12.1. HABIT Executable Modules............... 141

12.2. HABIT Data Entry Routines............. 142

12.3. The HABIT Computational Routines............. 143

13. The Computational Time Steps Used in HABIT...... 145

13.1. CONHAB Time steps...................... 145

13.2. FPFP_2 Time steps................... 147

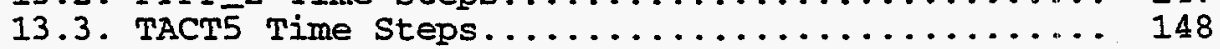

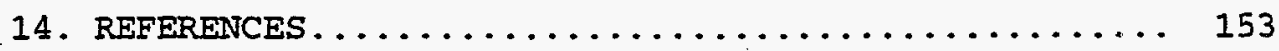


Figure 1.1: Schematic Diagram of Flow into the

Control Room as Modeled in HABIT................ 10

Figure 3.1: The File Name Window As It Appears when Invoked from the Design Edit Window Within the HABIT Main Window.......................

Figure 3.2: The File Path Window As It Appears when Invoked from the Design Edit window within the

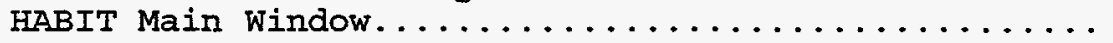

Figure 4.1: The HABIT Main Window as it Appears When the Use Same Data for Filtered Paths $1 \& 2$ Box is

Not Checked............................

Figure 4.2: The HABIT Main Window as it Appears when the Use Same Data for Filtered Paths 1 \& 2 Box is Checked.............................

Figure 5.1: The Design Edit Window As it appears from the HABIT Main Window.................... 26

Figure 6.1: The EXTRAN Design Form when Spill Parameters are being input.................

Figure 6.2: The EXTRAN Design Form When Meteorological Parameters are being input.........

Figure 6.3: The EXTRAN Design Form When Chemical Parameters are being input................ 52

Figure 7.1 : The CHEM Main Form............... 59

Figure 7.2 : The Change Units for CHEM form......... 60

Figure 7.3: The CHEM Control Room Flow Parameters

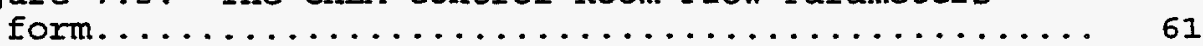

Figure 8.1: The TACT5 Release Design Form......... 76

Figure 8.2: The TACT5 Nuclide selection Form....... 77

Figure 8.3: The TACT5 Isotope selection Form (the

Figure 8.4 : The TACT5 Node Design Form........... 79

Figure 8.5: The TACT5 Plant Parameter Form......... 80 
Figure 8.6: The TACT5 Time Dependent Variables Form...

Figure 8.7: The TACT5 Time Dependent Variable Input Form. Shown for Initial Activity (fraction), independent of isotopic group................

Figure 9.1: A Schematic Diagram of the Flow Used in

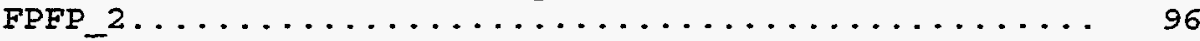

Figure 9.2: The FPFP_2 Release Design Form......... 97

Figure 9.3: The FPFP 2 Space Design Form as it

Appears for a Room....................... 98

Figure 9.4: The FPFP_2 Space Design Form as it Appears for a Duct.......................

Figure 9.5: The FPFP_2 Space Design Form as it Appears for a Filter...................... 100

Figure 9.6: The FPFP_2 Space Design Form as it Appears for a Resistance................... 101

Figure 9.7: The FPFP 2 Flow Design Form as it Appears

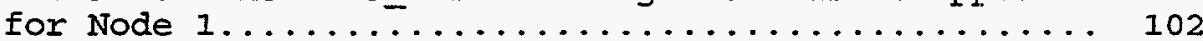

Figure 9.8: The FPFP 2 Flow Design Form as it Appears

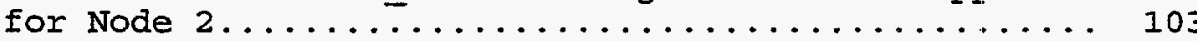

Figure 9.9: The FPFP 2 Flow Design Form as it Appears for Node 3 (the end node) ................... 104

Figure 10.1: Schematic Diagram of the Flows used in

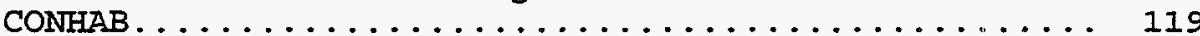

Figure 10.2: The CONHAB Main Form.............. 120

Figure 10.3: The CONHAB Plant Parameters Form....... 121

Figure 10.4: The Change Units for CONHAB Form....... 122

Figure 10.5: The CONHAB Meteorological or X/Q Data Form when Meteorological Data are Being Input...... I23

Figure 10.6: The CONHAB Meteorological or $x / Q$ Data Form when $x / Q$ Data are Being Input............. 124

Figure 10.7: The CONHAB Control Room Flow Parameters

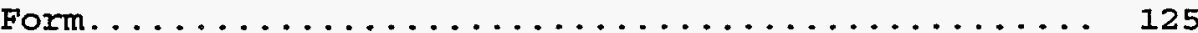

Figure 11.1: Schematic Diagram of the Flow used in DEMO1............................... 129

Figure 11.2: Concentration Versus Time for DEMO1..... 130 
Figure 11.3: Schematic Diagram of the Situation Modeled in DEMO2...................... 134

Figure 11.4: Schematic Diagram of the Containment Flows Used in TACTS for DEMO2 .............. 135

Figure 11.5: Schematic Diagram Control Room Flows

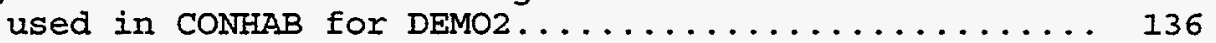

Figure 11.6: Rem/hr exposure within the Control Room and at the Intake for DEMO $\ldots \ldots \ldots \ldots \ldots \ldots \ldots \ldots \ldots$

Figure 11.7: Schematic Diagram of the situation

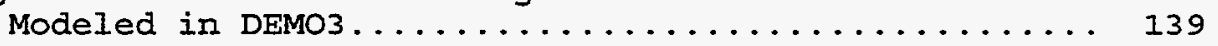

Figure 11.8: Schematic Diagram of the way DEMO3 is Modeled by FPFP_2....................... 140

Figure 13.1: Schematic Diagram of Rule 1 for Determining Time steps................... 149

Figure 13.2: Schematic Diagram of Rule 2 for Determining Time steps................... 150

Figure 13.3: Schematic Diagram of Rule 3 for Determining Time steps................... 


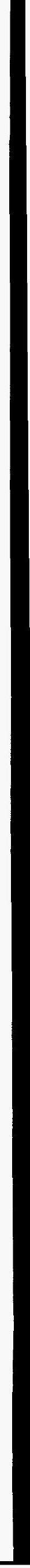




\section{IIST OF TABLES}

Table 6.1: Maximum Noon Solar Radiation Flux

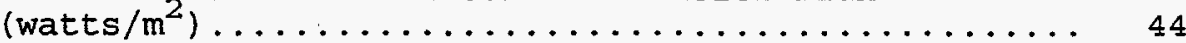

Table 6.2: Average Solar Radiation (sr) in Watts $/ \mathrm{m}^{2}$ and Cloud Cover (cc) in tenths at the Hanford Meteorology station. Based on data from Ramsdell

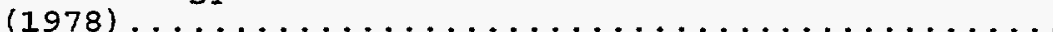

Table $6.3:$ EXTRAN work sheet.................. 49

Table 9.1: Physical Processes Modeled in FPFP_2..... 85

Table 9.2: Data Needed for FPFP_2 Spaces........... 95 


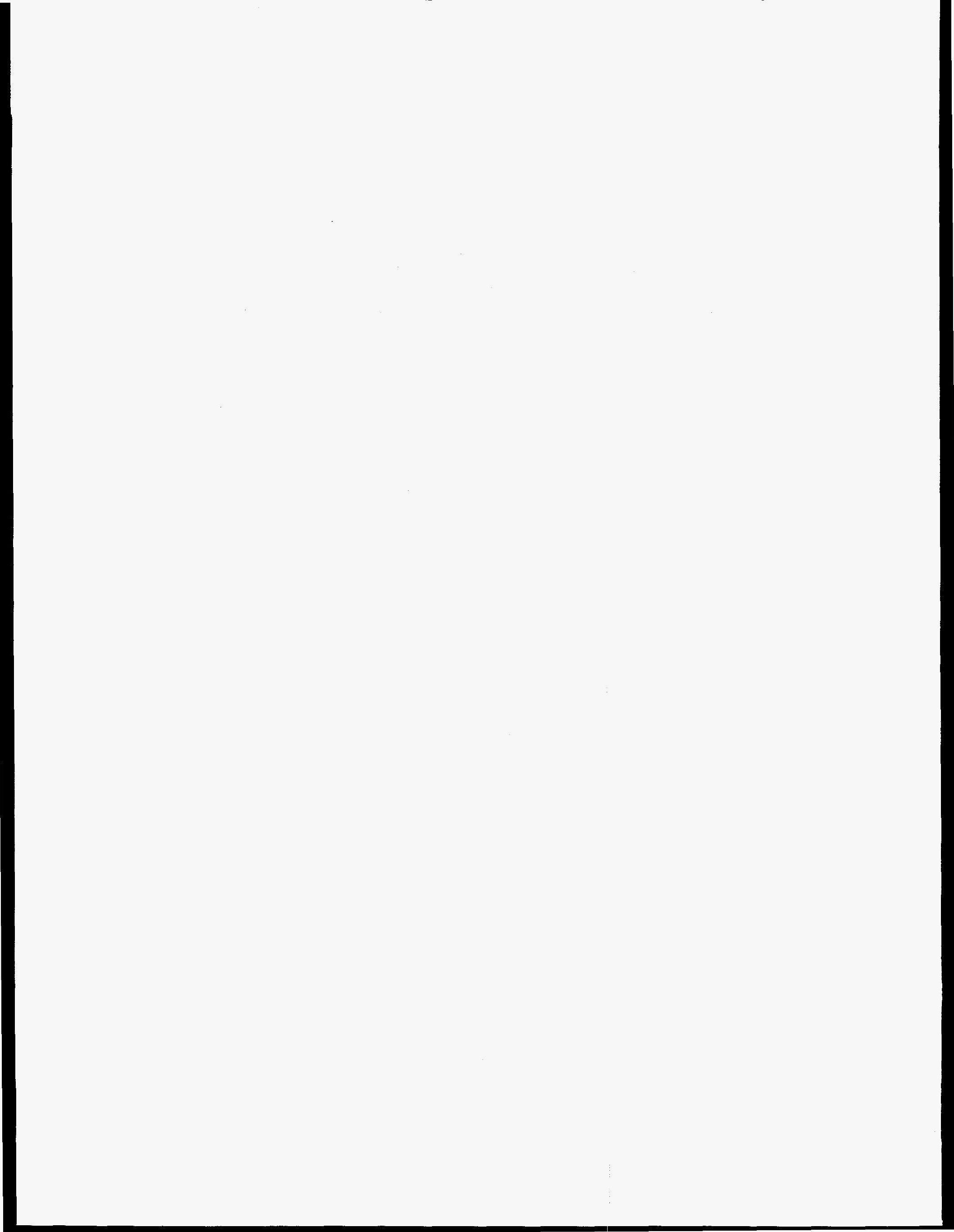


The Computer Codes for Evaluation of Control Room Habitability (HABIT) is a package of computer codes designed to assist in the evaluation of control room habitability in the event of accidental spills of toxic chemicals or the accidental release of radionuclides.

Several computer codes previously existed that were capable of performing parts of the computations needed to estimate chemical exposures or radiological doses in control rooms. The Pacific Northwest Laboratory (PNL) was given the task of combining these modules into a unified package that could be used with a maximum of ease and a minimum of user preparation. Data input has been completely redesigned. Improvements have been made in some of the computational methods used in the modules. The programs produce files containing tabular output and other files that can readily be imported into commercial spreadsheets to be graphed or for use in further computations.

Two modules are used for estimating chemical exposures. The first, EXTRAN, determines the release rate of a chemical in the event of release due to leaks or ruptures of liquid or gas tanks. It also uses a model that computes atmospheric dilution, including the effects of building wakes, to determine the chemical concentration (toxic and/or explosive gaseous materials) arriving at the intake to the control room. The second code, CHEM models the dilution of the chemical by flows in the control room and determines the chemical exposure to control room personnel.

Three modules can be used to determine doses in the control room associated with the accidental releases of radionuclides: TACT5, FPFP 2, and CONHAB.

The TACT5 code is used to model situations in which contamination is released to the atmosphere and subsequently enters the control room. The model allows the plant to be modeled as a group of compartments, which are called nodes in the nomenclature of TACT5. Air exchange can occur between any pair of nodes and it is possible for air to escape from any node into the atmosphere. The transfer may be filtered or unfiltered and the nodes may contain sprays. TACT5 may be used to produce atmospheric release rates for use by CONHAB for determining control room doses and can also be used by itself to estimate doses at three selected exterior points.

The FPFP 2 code is used to model radioactive flow through the plant. The Elow path is Ereated as a linear sequence of flow elements, which are called spaces and nodes in FPFP 2. The flow path leads to the control room intake. The spaces may be rooms, ducts, filters, or resistances. One node exists between each pair of spaces. These nodes are points at which clean air may flow into the path from the environment or contaminated air may escape to the environment. The code includes the effects of removal by sprays, filters, gravitational settling of particles, and diffusion of iodine onto surfaces.

The CONHAB code can accept contaminant inflow from up to two sources of outside air (i.e., two TACT5 runs) and up to two sources of air from within the plant (i.e., two FPFP_2 runs), CONHAB includes the effects of radioactive decay, dilution by contrō room flows, and removal by filters and produces doses to control room personnel. 



\section{ACKNOWLEDGEMENTS}

This report was prepared for the Division of Systems Technology, office of Nuclear Regulatory Research, of the U.S. Nuclear Regulatory Commission. The NRC Project Manager was Charles Ferrell who provided comments and suggestions on the use of the EXTRAN code to evaluate the concentrations of accidentally released flammable/explosive gasses as well as toxic materials in accordance with Regulatory Guide 1.91, "Evaluation of Explosions Postulated to Occur on Transportation Routes Near Nuclear Power Plant Sites". Jason Schaperow at the NRC provided extensive management and technical assistance throughout the project, incluaing several calculations used in verification of the program results. He also gave numerous helpful comments on the manuscript. $J . V$. Ramsdell was the PNL project manager during the first half of the project.

The author is also indebted to the many programers and authors who wrote the original codes that have been incorporated into HABIT and the associated documentation. As explained in the text, significant portions of this document have been taken from that work.

Any mention of products made in this document does not constitute an endorsement of those products by the United States Government, Battelle Memorial Institute, Pacific Northwest Laboratories, or by the author.

MS-DOS, Visual Basic, Visual Basic for DOS, and Microsoft Fortran are registered trademarks of the Microsoft Corporation. 


\author{
1. AN OVERVIEW OF THE \\ CONTROL ROOM HABITABIIITY PACKAGE \\ (HABIT).
}

\title{
1.1. A Brief Description of HABIT.
}

The Control Room Habitability Package (HABIT) is an integrated set of computer programs designed to estimate the radiation doses and chemical exposures that personnel in the control room of a nuclear facility would be exposed to in the event of an accidental release of radioactive materials or toxic chemicals.

HABIT consists of a number of program modules. Data entry routines use windows designed to guide the user through the correct sequence of steps and to allow easy review and editing of the data. When each value is entered, it is checked to be sure that it is within the predetermined range of reasonable values for that parameter. The computational routines are executed by clicking buttons on the data entry screens.

The computational routines produce files containing tabular output that can be printed or can be viewed using any common text processor. They also produce files that can be easily imported into spreadsheet programs using the "Import quote and comma delimited file" commands available on most commercial spreadsheets. The spreadsheet may then be used to graph results or to make further computations. Files are also used to convey results from one computational component to the next.

The major modules within HABIT are the HABIT Main Window, which controls the execution of the other components, and EXTRAN, CHEM, TACT5, FPFP_2, and CONHAB. Each of these modules is used to calculate a specific component needed for estimation of control room doses and exposures. They also each produce results that may be of interest in their own right.

Two program modules are run in sequence for chemical concentration and exposure computations. EXTRAN (for EXternal TRANsport of toxic chemicals) is the code for the computation of atmospheric chemical concentrations associated with a release of a toxic chemical. The EXTRAN code can be used to calculate the concentrations of flammable/explosive gaseous materials such as liquefied petroleum gas (LPG), hydrogen, and ethyl benzene from accidental releases near reactor sites. These three chemicals are included in the EXTRAN inventory of hazardous materials. The CHEM program uses the results of EXTRAN to determine the associated chemical exposures in the control room.

To make radionuclide dose computations, FPFP 2 and TACT5 are first run to calculate concentrations entering the control room and then CONHAB is run to calculate the control room concentrations and doses. The Fission Products Flow Path (FPFP_2) Program is used to compute the concentrations flowing through a path entirely within the plant (i.e., not traveling through the atmosphere) and entering the control room. The FPFP_2 code was developed to simulate conditions which existed at Three Mile Island Unit 2 in which postLOCA sump water entered the auxiliary building and into drains of the fuel handling building to produce an airborne source of radioactivity which diffused through rooms, doors, drain lines and other components to reach the reactor control room. Only by the use of the exhaust fans of the filtered ventilation systems to reduce the atmospheric pressure in these two buildings 
HABIT Manual

NUREG/CR-6210

Overview of HABIT

was the radiation level reduced in the control room. (Volume II Part 2 "THREE MILE ISLAND A Report to the Commissioners and to the Public " Mitchel Rogovin et al. Page 360 section $\mathrm{g}$. Mitigation of Releases of Radioactive Materials.)

The TACT5 (for Transport of ACTivity) Program is used to calculate the flow of nuclides through a group of nodes in a plant and the associated release rates of activity of each nuclide to the atmosphere. Each nuclide is partitioned into three chemical forms; elemental, organic, and particulate. TACT5 also computes doses, by organ, for up to three locations outside the plant, usually the exclusion radius, the low population zone boundary, and one other receptor point.

The CONHAB module is used to actually compute doses in the control room. Figure 1.1 is a schematic diagram of the flows used in modeling the control room. Air may enter the control room from other parts of the plant through the two paths labeled "Unfiltered Path I" and "Unfiltered Path 2 ". Note that these labels are used, even though there may be filters in these paths, to preserve the nomenclature used in previous versions of CONHAB. Concentrations entering through each of these two paths are obtained from the output of a run of FPFP 2. Air may also enter through the atmospheric intake labeled "Filtered Flow Path 1" containing concentrations obtained from the output of TACT5. Air may flow out of the control room, through a recirculating filter, then return to the control room. Ieaks of exterior air into the ducts feeding the recirculating filter may be modeled using the path labeled "Filtered Flow Path 2, Feeds Recirculating Filter". Concentrations entering this flow path are obtained by making a run of TACT5 for this path. Finally, clean bottled air may enter the control room.

\subsection{The History of HABIT.}

The computer codes for EXTRAN, CHEM, FPFP_2, TACT5, and CONHAB were developed separately and have been documented in the references mentioned elsewhere in this report. Each of these components could have been used by itself, but putting the pieces together to evaluate control room habitability required considerable intervention by the user including manual editing of files. Also, the data entry routines were laborious to use. Changing data to correct errors or to determine the effect of a change in release scenario or plant design required either reentering all data for a run or manually editing the input files.

The Pacific Northwest Laboratory (PNL) was given the task of designing new, more user-friendly data input routines for these programs and of combining the pieces into an integrated, easy-to-use package for evaluating control room habitability. Expressed goals of the project were to increase the ease of making computations and reduce the amount of user training required.

\subsection{The structure of this Document.}

This chapter gives an overview of the HABIT package, tells how to obtain HABIT, how to install it, and how to start it. Chapter 2 gives some general instructions for using HABIT, including how to use the mouse or keyboard to enter data and make runs. Chapter 3 describes the use of files by HABIT. The first part of the chapter explains the conventions that HABIT uses for naming files and the second part tells how to use HABIT's file path and file name windows. Chapter 4 explains the use of the HABIT Main window. Chapter 5 explains the use of the Design Edit window, which is used to start new designs, to edit old ones, and to copy, move or delete designs. 
Chapters 6 through 10 discuss EXTRAN, CHEM, TACT5, FPFP_2, and CONHAB respectively. Each of these chapters begins with a general description of the module, followed by an explanation of the technical basis for the program, then gives details of the windows used to enter data and run the program. Forms for preparing data to be entered into the programs are given at the end of each chapter.

Chapter 11 discusses three sample runs that are provided on the HABIT distribution disks and may be used to verify that HABIT is running correctly on your system or as tutorials for HABIT.

Chapter 12 contains programming notes for HABIT. Most users can skip this chapter, but it may be helpful to anyone who wants to modify HABIT, or who needs to take a detailed look at how the codes work.

Chapter 13 tells how HABIT determines the time-steps size to use to obtain good computational accuracy.

\title{
1.4. Before starting the HABIT Programs.
}

The first step in making a control room habitability calculation is to review the design specifications for the facility in question and to translate them into data that may be input into the HABIT program. This step requires understanding of the plant design, understanding of the engineering assumptions made by the various modules in HABIT, and the ability to convert the design specifications into a form that will enable HABIT to make exposure and dose estimates. As with any engineering model, it is wise to understand the assumptions and limitations of the model and to carefully consider whether the model assumptions are physically realistic for the plant design being evaluated.

Data preparation forms are included for each of the HABIT modules to aid the user in preparing to enter designs into HABIT.

\subsection{Obtaining HABIT.}

The Computer Codes for Evaluation of Control Room Habitability (EABIT) package is available by contacting:

\author{
Energy science and Technology software Center \\ P. O. BOX 1020 \\ Oak Ridge, Tennessee 37831-1020 \\ Internet: ESTSC@ADONIS.OSTI.GOV \\ Phone: (615)576-2606
}

The distribution package consists of a copy of NUREG/CR-6210 and computer disks containing the files needed to run HABIT, the source files for HABIT, and input and output files for the demonstration runs. 
HABIT Manual

NUREG/CR-6210

Overview of HABIT

\subsection{Installing HABIT.}

HABIT is designed to be run on a personal computer operating with DOS 5.0 or higher. It can also be run in a DOS window from either windows or $0 S / 2$. The computer must be have a video display that is capable of displaying 50 lines of text on the screen. A math coprocessor is highly desirable to speed computations, but the programs will run without one.

Disk space required is:

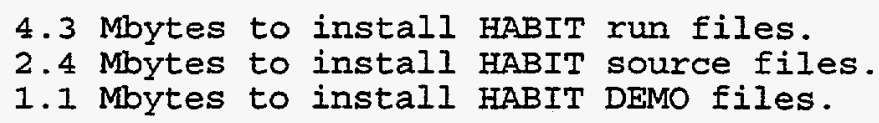

Before attempting to install HABIT, read the file README on HABIT Run Files Disk 1. This file may contain information about the version of HABIT on your disks that supersedes information in this NUREG/CR.

To install HABIT, place "HABIT Run Files Disk Number 1 " in your drive and type:

FromDrive:HINSTALL Frombrive ToDrive

where

Frombrive is the drive from which to copy the HABIT distribution files. (Usually a:)

ToDrive is the drive on which HABIT is to be installed.

(Usually c:)

Thus, most users will want to type:

a:HINSTALL a: c:

You will be given the choice of installing:

1. HABIT run files.

2. HABIT source files.

3. HABIT DEMO files.

You should normally enter $C$ : as the drive on which to install HABIT. The install program will create the directories needed for HABIT. If you install HABIT on $\mathrm{C}$ : then the HABIT files will be located as follows:

HABIT run files on $\mathrm{C}: \backslash H A B I T$,

HABIT source files on $C: \backslash H A B$ SRC and its subdirectories, and

HABIT demonstration runs on $\bar{C}: \backslash H A B \_D E M O$ and its subdirectories.

If you wish to run HABIT under OS $/ 2$ you will need to edit HABIT.BAT so that it uses the correct MODE statement to set 50-line mode in $0 S / 2$. Instructions for doing this are in HABIT.BAT.

If you have installed HABIT on $C$ : \HABIT you are now done with the installation. If you have installed HABIT on a path other than C: \HABIT, you will need to edit several files so that they reflect the drive you have used. The files that need editing are: 


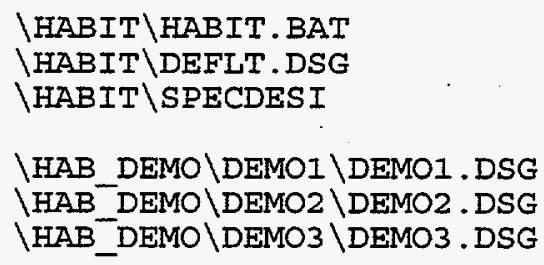

Globally change all occurrences of $\mathrm{C}: \backslash$ in these files to the path you have used.

This completes installation of HABIT.

1.7. Starting HABIT.

HABIT is started from the DOS prompt by typing:

$$
\text { C: } \backslash H A B I T \backslash H A B I T
$$

This invokes a batch file that sets the screen to 50-line mode, sets some needed environment variables, and then opens the HABIT Main Window. When HABIT is finished, it will set the screen to 25-line mode. If you want the screen left in 50-line mode, you may edit HABIT.BAT to remove the final mode command.

\subsection{Possible Errors.}

This section discusses how to remedy some errors that may possibly occur when running HABIT.

\subsubsection{Out of Memory Errors.}

Some of the HABIT routines dynamically allocate memory depending on the steps that the user takes. It is therefore not possible to state the amount of free memory required to run HABIT. However, experience shows that HABIT runs well on computers with over about $550 \mathrm{~K}$ bytes of memory available, may have trouble if the memory available gets below about 500K bytes. You may use the DOS MEM command to check the amount of memory available on your system. Note that HABIT runs in conventional memory.

Several different error messages can occur which indicate that there is insufficient memory to run HABIT. These include "Out of Memory",

"Insufficient Memory to Run...", "Out of Heap Space", "Out of stack Space", and others.

If you get one of these error messages you have two options. The first option is to try removing unneeded memory-resident programs and loading as many routines as possible into high memory. See the memory management sections of your DOS manual.

The second option, which is guaranteed to work, is to create a "clean boot" disk. Use the SYS command to create a bootable disk (see your Dos manual). Place a short version of CONFIG.SYS on the disk. It should contain only the following two lines:

DEVICE $=C: \backslash D O S \backslash A N S I . S Y S$

SHELL=C: \DOS COMMAND. COM /E: $1024 / \mathrm{P}$ 
HABIT Manual

NUREG/CR-6210

Overview of HABIT

Place this disk in your disk drive and reboot the system. You will then have sufficient memory to run HABIT. You will need to reboot the system from this disk before each HABIT session.

\subsubsection{Incorrect Output of Time steps.}

When the computational modules of HABIT are run, they are supposed to write the time of each time step to the screen. The time for each step is supposed to be written at the same place on the screen. If you find that the time is written in a disorderly fashion across the page with some characters such as $\leftarrow$ [u written before each time, it is a sign that you do not have the ANSI.SYS driver installed. The programs will run fine except that the time is not written to the screen in the manner intended.

If you have this problem and wish to correct it, you may do so by placing the following line in your CONFIG.SYS file:

$$
\text { DEVICE }=C: \backslash D O S \backslash \text { ANSI . SYS }
$$

Then reboot your system. If your Dos files are on a directory other than C: \DOS $\backslash$, you will need to change the above line accordingly. Consult your Dos manual for more information about installing ANSI.SYS.

\subsubsection{Out of Environment Space.}

HABIT may not run correctly if you receive the message "OUT OF ENVIRONMENT SPACE" when you run HABIT.BAT. There are a few ways that you can correct this problem if it occurs.

1. Use the SET command to check environment variables and eliminate some that are not needed.

2. Temporarily load a version of COMMAND.SYS with more environment space by typing:

COMMAND /E: 1024

Then run HABIT. If the error persists try using /E:2048.

3. A more permanent $f i x$ is to insert the following line in your CONFIG.SYS file:

DEVICE=COMMAND. COM /E: $1024 / P$

Then reboot your system. For more information on environment space, consult your DOS manual.

\subsection{Verification and Validation}

HABIT combines five computer codes previously published by the Nuclear Regulatory Commission into a single package. Combination of the codes within a unified package is an attempt to make the programs easier to use. It has been necessary to modify portions of each of the codes to achieve this result. In general, the modifications have been limited to those portions of the programs related to input and output. These modifications, and the data input and output portions of the HABIT shell, have been thoroughly checked to ensure accurate transfer of information among the programs. 
The computational portions of EXTRAN (Ramsdel1 1991) and CHEM (Gilpin 1990), the two computer programs used to evaluate control room habitability following postulated releases of toxic materials, were not modified. The results of EXTRAN calculations within HABIT are identical to results from the version of EXTRAN that was used as the starting point in development of HABIT. These results produced by the original code were verified to be numerically correct. The computational results of the CHEM code have been verified by comparison with analytical solutions to the differential equations being solved by the code.

The three remaining codes, TACT5 (West and Gilpin 1988), FPFP_2 (OwCarski, et al. 1991), and CONHAB (Gilpin 1990), are used to evaluate confrol room habitability following postulated releases of radioactive material. The computational results of TACT5 within HABIT have been compared with results from the original version of the code and are identical. It was found that the numerical techniques previously used in FPFP 2 and CONHAB could give poor accuracy in situations with large time steps. These algorithms have been revised to ensure good accuracy and then tested by comparison with hand calculations and with runs in which the input file specified many small time steps.

With two exceptions, the physical assumptions made in HABIT are identical to those used in the original versions of the component programs. The only exceptions are the spray model now used in FPFP 2, and the building wake model now used in EXTRAN and CONHAB. Justifications for the physical assumptions and modeling are given in the respective NUREG/CR's for the computer codes.

The original spray routine in FPFP 2 gave unrealistically high spray removal rates. The HABIT version of FPFP $\overline{2}$ uses the equations for spray removal coefficients published by powers, et al. (1993) and is based on experimental data cited in that work. The new FPFP_2 spray routine gives physically reasonable values. It has been verified by comparison of the computational results of the code within HABIT with results of hand calculations using the equations published by Powers, et al.

The second change in physical assumptions is that the atmospheric dispersion algorithms in EXTRAN and CONHAB were modified to use the formulation of Ramsdell (1995). These modifications were checked by hand calculations, by comparison of the EXTRAN and CONHAB codes, and by comparison of results from the two codes. The Ramsdell (1995) model uses more realistic physical assumptions than previous models to account for building wake effects and plume meander. It gives a better fit to data while continuing to produce conservative estimates. As explained by Ramsdell (1995), it has undergone peer review.

\subsection{Isotope Data Files Used by HABIT.}

The HABIT computer codes can use several data files containing physical data for isotopes. The files ICRP.02, ICRP.30, MLWRICRP. 02 and MLWRICRP.30 are identical with those used in previous versions of TACT5 and CONHAB. The files MLWRFGR. 11 and FGR.11 were generated by the NRC staff for use in HABIT. The following text, supplied by Jason schaperow, describes these data files:

\section{TACT5 Code}

The HABIT version of the TACT5 code is used to calculate release rates of radioactivity to the environment and offsite doses resulting from a 
postulated design-basis LOCA. Required input is a source term, the isotopes that comprise it, and the associated physical data for each isotope.

DiNunno (1962) gives the TID-14844 source term and the isotopes that comprise it. The computer files MLWRICRP.02 and MLWRICRP.30 (West and Gilpin, 1988) give the associated physical data, that is, dose conversion factors, radioactive decay constants, and Ci/MWt. These files each contain physical data for 122 isotopes, which include the isotopes in the DiNunno source term. The dose conversion factors in these files are taken from ICRP Publication 2 (International Commission on Radiological protection, 1959) and ICRP Publication 30 (International Commission on Radiological Protection, 1979). ICRP publication 30 contains more up-to-date models and physical data than ICRP Publication 2; therefore, MLWRICRP. 30 is currently used by NRC for analysis.

Soffer, et al. (1994) gives a proposed new source term and the elements that comprise it. MLWRICRP. 30 was reviewed to determine whether it contained the physical data needed for analysis using the new source term. The following are the results of that review:

Isotopes: Soffer, et al. (1994) gives the elements that comprise the new source term, but does not list the important isotopes of these elements. However, Chanin, et al. (1990) lists the 60 important isotopes of these elements. Therefore, MLWRICRP. 30 was compared to the 60 isotopes in Chanin, et al. (1990). MLWRICRP.30 does not contain four of the 60 isotopes, that is, Co-58, Co-60, $\mathrm{Rh}-105$, and Sb-129. To correct this problem, the file MLWRFGR.11 was created. MLWRFGR.11 contains the isotopes in MLWRICRP.30 plus the four additional isotopes.

Dose Conversion Factors: MLWRICRP.30 contains dose conversion factors for two pathways and six organs. These are submersion in a semi-infinite cloud (whole body and skin) and inhalation (thyroid, lung, bone, and liver). A review of the dose conversion factors in this file showed that many of them were set to "0.0." To correct this problem and have the latest dose conversion factors, the dose conversion factors in MLWRFGR.11 were taken from Federal Guidance Reports 11 and 12 (FGR 11, 1988 and FGR 12, 1993). (The bone dose conversion factors in MLWRFGR.11 are for bone marrow and not bone surface.)

Radioactive Decay Constants and Ci/MWt: For Co-58, Co-60, Rh-105, and Sb-129, MLWRFGR. I1 contains the decay constants and $\mathrm{Ci} / \mathrm{MWt}$ from Chanin, et al. (1990). For the remaining isotopes, MLWRFGR.11 contains decay constants and Ci/MWt from MLWRICRP. 30 .

\section{CONHAB Code}

The CONHAB code is used to calculate control room doses resulting from a postulated design-basis LOCA. Required input is the isotope names and release rates, which is the output from the HABIT version of TACT5, or the core fractions per cubic feet flowing into the control room through unfiltered paths, which is the output form the HABIT version of FPFP 2 . The associated physical data is also needed. The associated physical data consists of dose conversion factors, radioactive decay constants, $\mathrm{Ci} / \mathrm{MWt}$, and decay chain information. The computer files ICRP.02 and 
ICRP.30 (Gilpin, 1990) contain the associated physical data. ICRP.02 and ICRP. 30 consist of the physical data in MLWRICRP. 02 and MLWRICRP. 30 plus decay chain information.

Although the CONHAB code was not designed to be used with the new source term, it is desirable for CONHAB to have the latest dose conversion factors. Therefore, the file FGR. II was created. FGR.11 consists of the physical data in MLWRFGR.11 plus decay chain information from ICRP.30. For the additional four isotopes, decay chain information was taken from Lederer, et al. (1967).

\section{Additional Notes}

The decay chain information in FGR.II was also added to MLWRFGR.II.

Eight of the isotopes were removed from MLWRFGR. 11 and FGR. 11, since they are at the beginning of their decay chains and have short half lives (less than 10 minutes), (2) their dose conversion factors are not given in Federal Guidance Reports 11 and 12 , and (3) they are not members of the list of 60 isotopes in Chanin, et al. (1990). These eight isotopes are the following: $\mathrm{Br}-85, \mathrm{Kr}-89, \mathrm{Rb}-90, \mathrm{Sr}-93, \mathrm{Rh}-109, \mathrm{I}-$ 136, $\mathrm{Xe}-137$, and $\mathrm{Cs}-139$. 


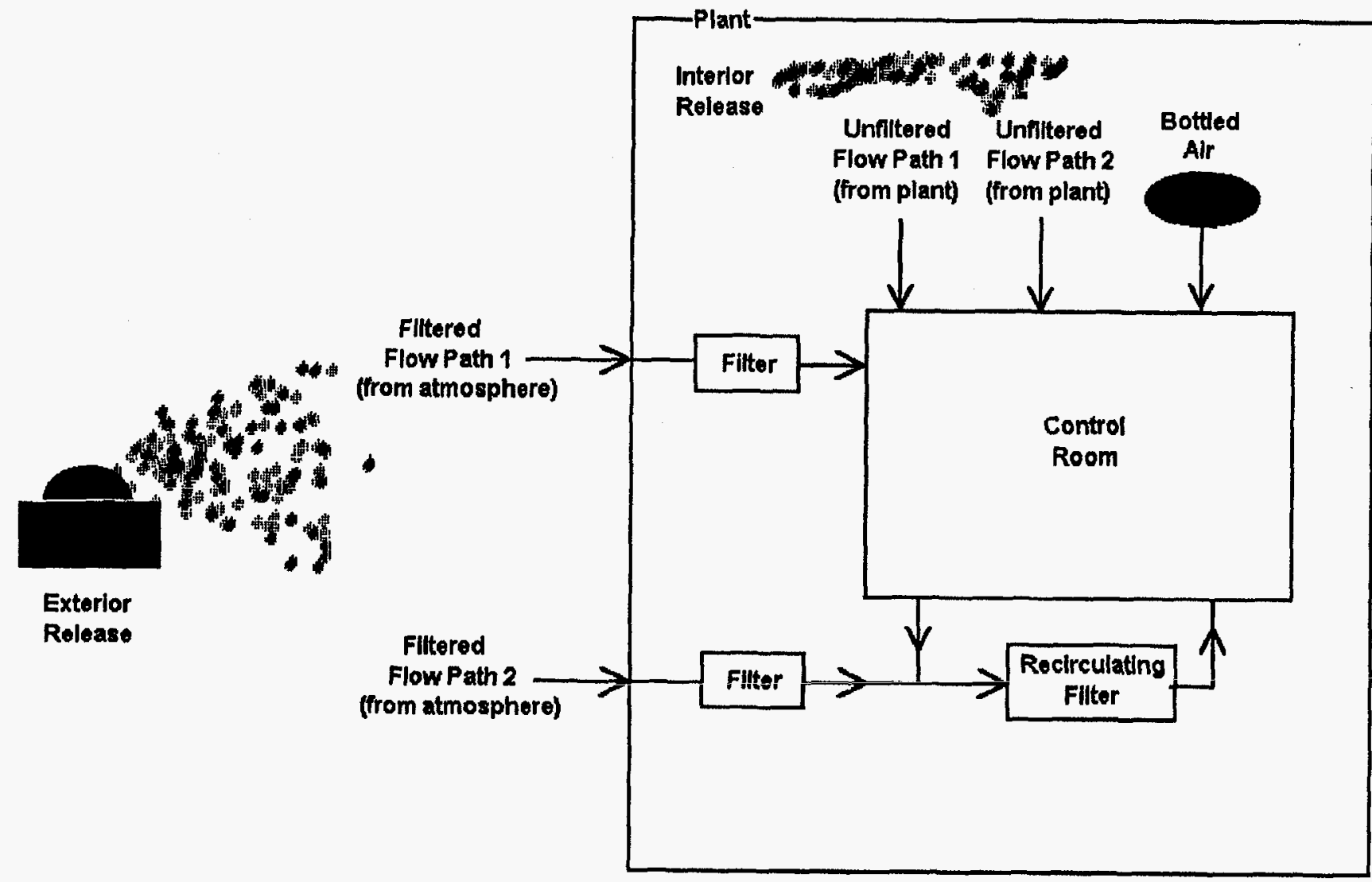

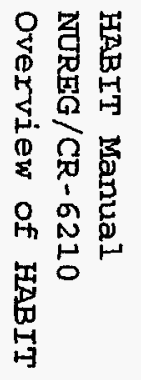

Figure 1.1: Schematic Diagram of FIow into the Control Room as Modeled in HABIT. 
The data entry routines in HABIT were written using Microsoft Visual Basic for DOS. The operation of the data windows will be familiar to anyone who has used a windows environment. All operations in HABIT can be performed using only the keyboard, however some operations are easier using a mouse. A set of basic instructions are given here. A person familiar with using programs that use windows can skip this section.

Data entry takes place in a series of windows, or forms, which appear as needed. The forms have been designed to visually suggest the order of operations that must be done to enter the data and make computations. Data entry usually proceeds from top to bottom and from left to right. HABIT is designed to be simple for the beginning user, but also to be powerful for advanced users. There are several controls on the HABIT forms that need only be used for advanced procedures.

This section of the manual is best read while sitting in front of a computer running $\mathrm{HABIT}$, so that you can experiment with the various operations.

Several types of objects, called controls are used. These include buttons, text entry boxes, value entry boxes, option buttons, check boxes, and drop down lists. There are several ways that the keyboard and the mouse can be used to operate these controls. The instructions given here are intended to allow the user to begin using HABIT, but are not intended to be complete. Consult a book on Visual Basic or a manual on any windows environment for full instructions. Users are encouraged to experiment to find the easiest way to perform tasks.

Control Buttons are used to initiate specific actions. Text entry boxes and value entry boxes are used to enter text and numerical values respectively. Option buttons are used to select one choice from a group of several options. Check boxes are used to mark zero or more items in a group. Drop down lists are used to select one item from a list.

\subsection{Using Hot Keys.}

"Hot keys" provide a rapid way to perform HABIT operations using only the keyboard. Nearly all buttons in HABIT have hot keys as do many other controls. A hot key is indicated by one white letter or digit in the text for a control. For example, the Edit Design button that appears on the main data entry window for each HABIT module has a white $E$. Holding down the <Alt> and then pressing the hot key clicks the corresponding control. In the case of the Edit Design Button hold down <alt> and press $\mathbf{E}$. This is often written in computer manuals as $\langle$ Alt $\rangle+E$.

The use of hot keys is highly recommended. A user with even a modest amount of experience will find that hot keys provide the most rapid means of performing HABIT tasks. Hot keys are generally even faster than clicking with the mouse. 


\subsection{Using the Mouse.}

To use the mouse to "click on" a control, position the mouse pointer on the button and press the left mouse button. HABIT does not use the right mouse button.

Click on a control button to begin the action performed by the button.

Click on a text box or a value box to place the focus in the box and then use the keyboard to enter the text or value.

Click on one of a set of option buttons to choose that option.

Click on a check box to mark the box with an $\mathrm{X}$ (thus indicating that the box is selected) or to remove the $x$ mark from a box (thus indicating that the box is not selected).

Click on the arrow at the right of a drop down list to cause the list to "drop down".

\subsection{Using the Keyboard.}

At any given time one of the controls on a form "has the focus". For most controls the focus is indicated by the location of the blinking underline cursor. For control buttons, the focus is indicated by black color surrounding the letters on the button.

The <Tab> key moves the focus to the next control and <Shift>+<Tab> (i.e., holding down the shift key and pressing the tab key) moves the focus to the previous control on the form.

Once a button has the focus pressing either <Entel:> or <Space> will click the button; i.e., cause the button to begin performing its task.

Once a text entry box or a value entry box has the focus, you are able to begin typing the text or value.

Once a group of option buttons have the focus, <up arrow> and <down arrow> can be used to change which button of the group is selected (indicated by a bullet between the parentheses for the button).

When a check box has the focus, <Spaces can be used to toggle the $\mathrm{X}$ in the check box on or off. $<+>$ can be used to mark the box with an $x$ and $<->$ can be used to remove the $X$ mark from $a$ box. When an $X$ appears in a check box, it indicates that the item represented by the box has been selected.

\subsection{Use of the Escape Key, <Esc>.}

The escape key, <Esc>, is a special key. This key can always be used to leave the current form and return to the previous form.

The escape key acts as the hot key for all Done buttons in HABIT.

\subsection{Use of Cancel Buttons.}

Many forms in HABIT have Cancel buttons. The cancel button allows the user to interrupt the current operation without making any changes in the data base. 


\subsection{Using Text Entry Boxes.}

When the focus is first set on a text entry box or a value entry box, the contents of the box are seen in reverse video indicating that they are selected. Pressing any letter or number key deletes the contents of the box and places the appropriate letter or number in the box.

Some simple editing is possible in text and value entry boxes. The editing features are those provided by Microsoft Visual Basic and are similar to those found in the Edit text editor that comes with Microsoft DOS. A brief statement of these functions is given here. Consult youx Dos manual for more details.

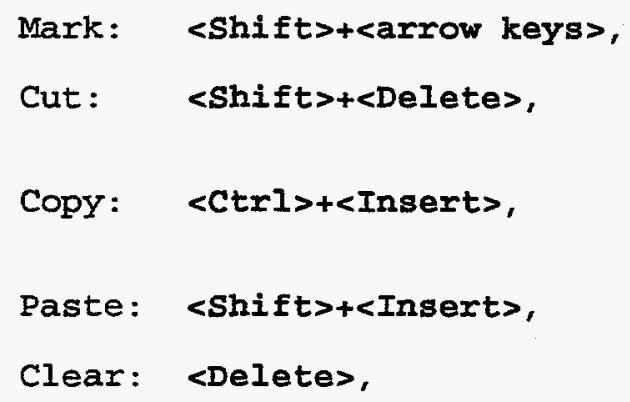

highlight text.

copy highlighted text to clipboard and delete it.

copy highlighted text to clipboard.

insert text from clipboard.

delete highlighted text.

An additional editing feature has been programmed into HABIT. If you press $\langle$ Ctrl $\rangle+\langle R\rangle$, then the text or value box will return to the value it had when it got the focus.

You can use <Home>, <End>, <left arrow>, and <right arrow> to move within the string.

Except in a few places, it is possible to enter strings up to $32 \mathrm{k}$ characters in text boxes. The text you enter will still be in the box even if it is too long to be visible at one time.

\subsection{Using Value Entry Boxes.}

Value entry boxes function just like text entry boxes except that the string entered is converted to a numerical value. Values may be entered in either floating point or exponential format.

\subsubsection{Blank Values.}

A blank value box indicates that no value has been entered for that variable. This is NOT the same as a value of zero. You may reset a value to "blank" by deleting all characters in the value box.

The HABIT data entry routines use a value of -999.0 internally to store blank values. Blank values are saved in the *. INP files as -999 or as the string "00.0", depending on the program.

\subsubsection{Range Checking.}

HABIT checks the values you enter to be sure that they are within the range of values considered reasonable for that parameter. Values are checked after the number has been entered when the focus is moved to another control. If you 
enter a value that is outside the allowed range, a message will appear showing the allowed range. You will be asked if you want to override the range check and use the value anyway or if you want to reenter the value. If you choose to reenter the value the focus will return to the value entry box in question and its value will be restored to its previous value.

To set the allowable minimum and maximum values for each variable, the HABIT data entry routines read the file c: lhabit/ranges.get. If a file named ranges.set exists on the current DATAPATH directory, then values are also read from that file and take precedence over values in the file on the c: \habit lranges.set file. It is thus possible to specify ranges for a specific design that are different form those used in other HABIT designs.

The RANGES.SET files are ASCII files and may be edited if it becomes necessary to change the allowable ranges. Comments in that file make it easy to see how to make the needed changes.

\subsection{Using Drop Down Lists.}

Drop down lists are used in a few places in HABIT. The drop down Iists can be recognized by a down arrow that appears at the right of the list. Clicking on the down arrow with the mouse causes the list to "drop down" so that several items in the list can be seen at once. <Alt>+<down arrows can also be used to make the list drop down. Whether the list is dropped down or not, <up arrow> and <down arrow> can be used to highlight items in the list. <Alt>+<up arrow> or clicking on another control causes the drop down list to cease being dropped down.

Within HABIT, changing the highlight in a drop down list causes other boxes on the data entry forms to change value to correspond to the highlighted list item.

\subsection{Using Help.}

Except for the figures, this entire NUREG/CR is available as an on-1ine help file. Nearly all windows in HABIT have Help buttons. When one of these buttons is clicked, a window is opened containing information about the window containing that Help button. The user may use the buttons on the Help window to search for help on other topics.

Page Up, Page Down, End, and Home may be used to move through the text.

The Prev button moves to the previous Help topic (i.e., the previous section of the NUREG/CR) and the Next button moves to the next topic.

The Contents button produces a list of topics sorted alphabetically.

Clicking Table of Contents on the comments list produces a list of topics sorted in the order they appear in the NUREG/CR.

The Help window may be resized by dragging the mouse on one of the corners of the window.

Unfortunately, subscripts and superscripts in the text appear in the help file on the same line as the text. This makes it difficult to read some of the equations in the help file. 


\section{THE USE OF FILES BY HABIT}

\subsection{HABIT File Naming Conventions.}

Two file paths are used by HABIT. The first of these is the path containing the executable files for the HABIT routines and other files needed to make runs of HABIT. This path, which is generally $c$ : \habit, is set in HABIT.BAT using the environment variable EXEPATH. The various HABIT routines check the environment variable EXEPATH to determine the path to use when looking for these files. For the sake of brevity, c: \habit is used throughout this NUREG/CR even though the user may have installed HABIT to another directory (See section 1.6).

The second path used by HABIT is DATAPATH. This is the path containing files for the design currently being used. Information about the current design is read from the file c: \habit\SPECDESI.

The HABIT package uses a rigid file naming convention. Filenames are based on a 5 character code that identifies the release design, followed by 2 or 3 characters that identify the program using the file, and, finally a file extension that identifies the contents of the file.

When you click the "Edit Design" button found on any of the HABIT main windows, and then click "New Design" you will be asked for the path to use for the new design. You have complete freedom in choosing a path for the design, however, it is a good idea to organize your designs in an orderly tree structure. You will probably want to use a separate subdirectory for each new design. If you specify a subdirectory that does not exist, you will be asked if you want to create the subdirectory. You will then be asked to specify up to 5 characters that identify this release design. If you specify less than 5 characters, then minus signs (-) will be automatically added to a total of 5 characters. Thus, if you enter "tst" the design id will be "tst--".

Except for a few invisible scratch files, all of the files used by HABIT are ASCII files that can be viewed using common text editors. The files contain comments indicating the contents. Although the user can use a text processor to edit the. INP files, it will generally be better to use the HABIT data entry windows to edit values in the your release designs. This is both easier and less likely to produce errors.

Throughout this NUREG/CR the string "ID---" will be used to refer to a filename having the ID of the current design. Thus, if this text mentions a file by the name ID-- T5A.CNX and you are currently working on a design with the id "DEMO-", the text is referring to the file DEMO-T5A.CNX.

For the release id "ID---", the files associated with programs are as follows:

$$
\begin{aligned}
& \text { ID---T5A.*, Files associated with TACT5 flow into filtered path } 1 . \\
& \text { ID---T5B.*, Files associated with TACT5 flow into filtered path } 2 . \\
& \text { ID---FPA.*, Files associated with FPFP_2 flow into unfiltered path } 1 .
\end{aligned}
$$




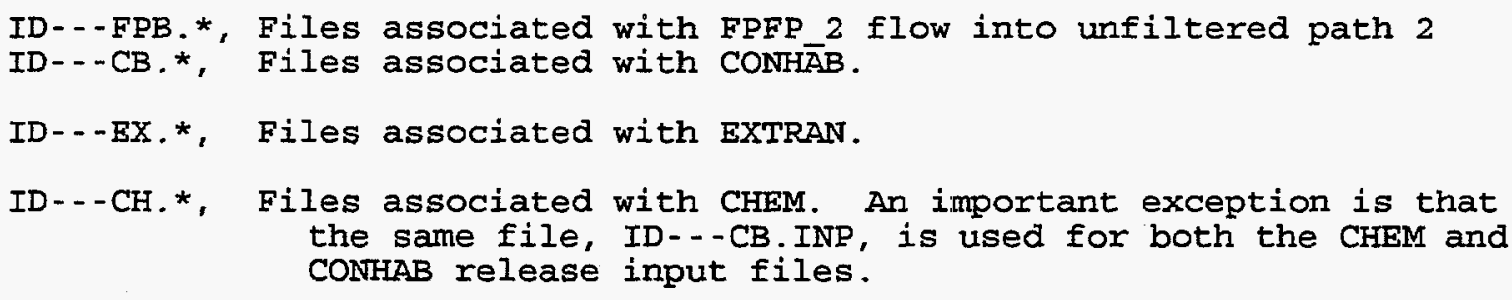

File extensions are used as follows:

*.INP, Release input files. These files are created by the data input routines and are read by the computational routines.

*.TAB, Files containing output tables. These are the files the user will be most interested in.

*.CNX, Files containing results to be transferred to the next program in the computational sequence. Some of these files may be of interest to the user. CNX stands for a connection file.

*.SPD, Files containing quote and comma-delimited output suitable for import to a spreadsheet. The user may import these into any of several commercially available spreadsheets for the purpose of graphing the results or of making further computations.

*.MB, Output file of mass balance numbers output by EXTRAN. This file may be of interest for examining the details of the evolution of the release.

*.DSG, The release design file. This file contains the names of all of the files that are (or may be) associated with this specific design. It is read whenever HABIT needs to determine the name of a file to use.

This file is created when the "New Design" button in the Design Edit window is clicked. The file C: \EABIT\SPECFILE is read and all occurrences of "DEMO" are replaced by the 5 character design id for this release design.

*.RUN, The files ID---*.RUN are created from ID---.DSG whenever a button is clicked to run one of the Fortran computational routines. It tells the Fortran program what files to read and write.

For example, the full list of .INP files for the design ID-.- is:

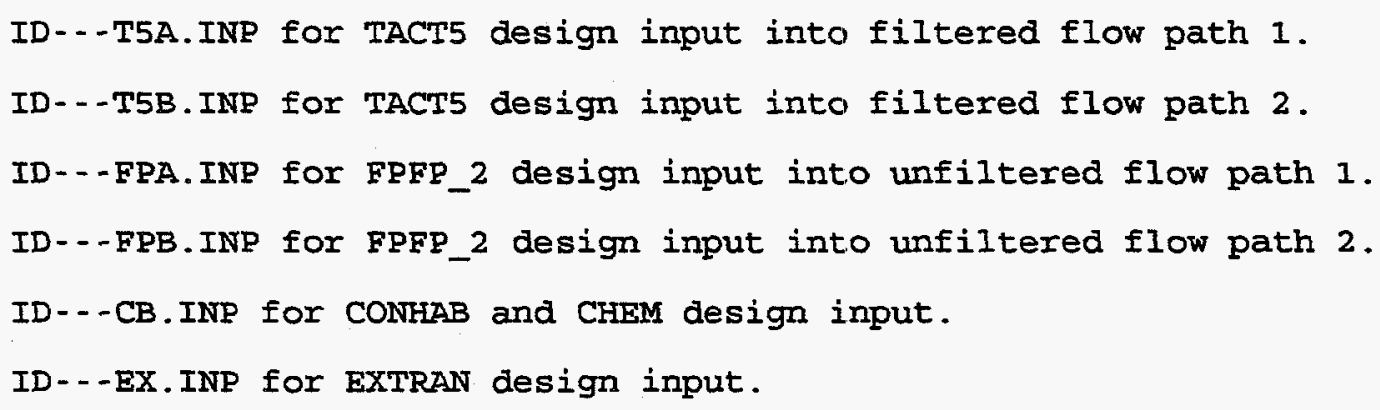


C: \HABIT\SPECFILE or any *.DSG file may be examined to help clarify the file names used by HABIT.

\subsection{Using the File Name and File Pathwindows.}

NOTE: Whenever the HABIT programs need to know the name of a file to read or write, the File Name window will appear. HABIT will suggest a file name. Unless you are an advanced user with a specific purpose in mind, you should simply push <Enter> or click the OK button to indicate that you want to use the file name suggested by HaBIT.

The File Name window (see Figure 3.1) is in a form that is similar to the file name forms used in Microsoft Windows and many other programs. It can be used in several ways to specify the name of a file. The simplest way is to type the name of the file in the file name text box and then press <Enter> or click the OK button to use that file name. The file name window can also be used to search for a file. The list of files will be updated whenever the drive, the path, or the file specification string is changed. Clicking a name in the file list inserts that name in the file name text entry box. "Double clicking" on a file name causes that file to be used.

The File Path window (Figure 3.2) is similar to the File Name window except that it is used to enter the name of a subdirectory to use as the path for a design. The path entered may be typed with or without the closing backslash

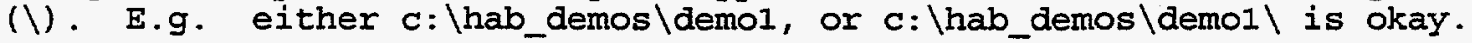




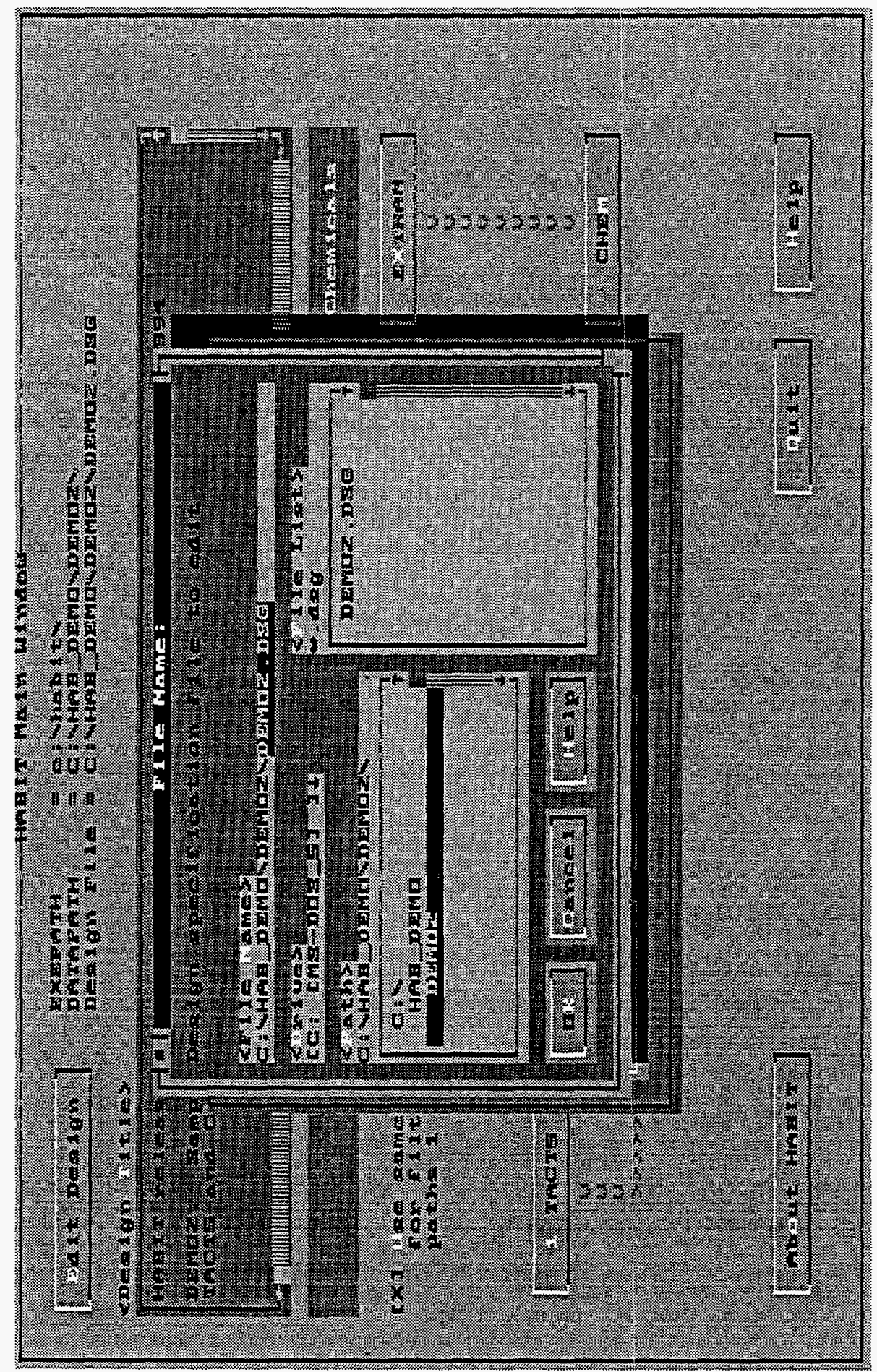

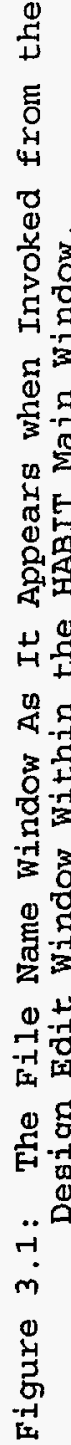




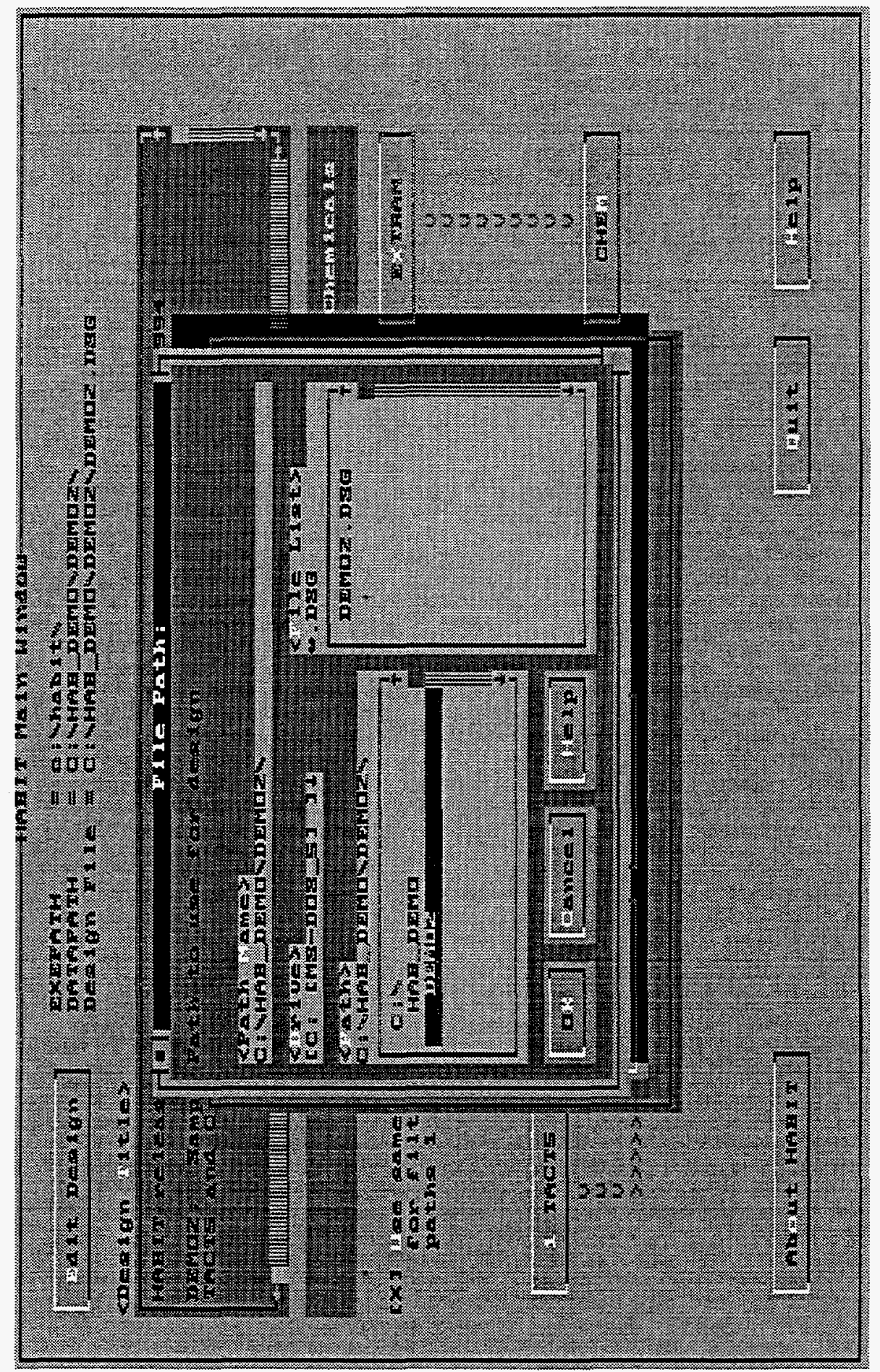

$\stackrel{0}{1}$

坖

7

응

点

年

密

in

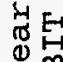

员寽

亖岳

先.

3돈

崫

:

동

$a_{1} 3$

$\underset{-1}{0}$

匠

跍告

.. 㟔

N

m

勇 



\section{THE HABIT MAIN WINDOW}

Figure 4.1 shows the HABIT Main Window. This window, which controls the overall execution of the components of HABIT, shows the logical layout of HABIT.

The upper part of this window is used to specify the design that is being entered or edited. The labels at the upper right of the window show the path for HABIT executable programs, the path for the release design currentiy being worked on, and a file name for the specific design currently being worked on. When HABIT is first installed, the program is set up to use a demonstration case. After that, HABIT remembers what design was used during the most recent HABIT session.

The following controls appear on this form:

The Edit Design button is used to create new designs, select an existing design, copy a design, or delete a design.

When you use the Create Design button, a file, ID---.DSG, is created that tells HABIT the names of the files to use for that design. These names are automatically set by HABIT using a set of conventions set in the file $c$ : \habit\SPECFILE. These names are automatically used whenever HABIT reads or writes a file. At various other times during data entry, HABIT will ask for the name of the file to use. Unless you are an advanced user with a specific purpose in mina, you should simply push <Enter> or click ok to use the name suggested by HABIT.

The Design Title text box is used to specify the title for the entire design. This title should be entered when a new design is begun so that it will appear in the titles for all of the computational runs within HABIT. If the design title is changed at a later time, the changes may not appear in some of the run titles.

The EXTRAN button is used to enter data and make a computational run of EXTRAN.

The CHEM button is used to enter data and make a computational run of CHEM. EXTRAN must be run before CHEM.

When the Use same data for filtered paths 1 \& 2 check box is not checked, the HABIT Main Window appears as shown in Figure 4.1 . In this case separate runs of TACT5 can be made for filtered paths 1 and 2 .

When this box is checked, the TACT5 button for filtered path 2 disappears and arrows are shown indicating that the same flow from TACT5 enters both filtered path 1 and filtered path 2 (See Figure 4.2). This indicates that the same TACT5 run should be used to determine the concentrations for filtered air intakes 1 and 2 . When this box is checked and the CONHAB button is clicked, the file ID---T5A.CNX (if it exists) is copied to ID-- T5B.CNX for use as input for filtered path 2.

The 1 TACT5 button is used to enter data and make a computational run of TACT5 for filtered path 1 . This is used to model nuclides released into the atmosphere which then enter the control room through a filtered 
intake.

The 2 TACT5 button is used to enter data and make a computational run of TACT5 for filtered path 2 (feeds recirculating filter). This is used to model nuclides released into the atmosphere, pass through a filter, then enter the ducting feeding the recirculating filter, and then pass through the recirculating filter into the control room.

The A FPFP_2 button is used to enter data and make a computational run of FPFP 2 to obtain concentrations flowing into the control room through unfiltered path 1 . If the air enters the control room through a filter, the filter should be considered as part of the flow path used in FPFP_2.

The B FPFP_2 button is used to enter data and make a computational run of FPFP 2 to obtain concentrations flowing into the control room through unfiltered path 2. If the air enters the control room through a filter, the filter should be considered as part of the flow path used in FPFP_2.

The CONHAB button is used to enter data and make a computational run of CONHAB. Before running CONHAB, TACT5 and FPFP_2 must be run for all of the paths included in the release design.

The About HABIT button is used to see information about HABIT.

The Quit button is used to end a HABIT session and return to DOS.

The Help button is used to obtain on-line help with HABIT. 


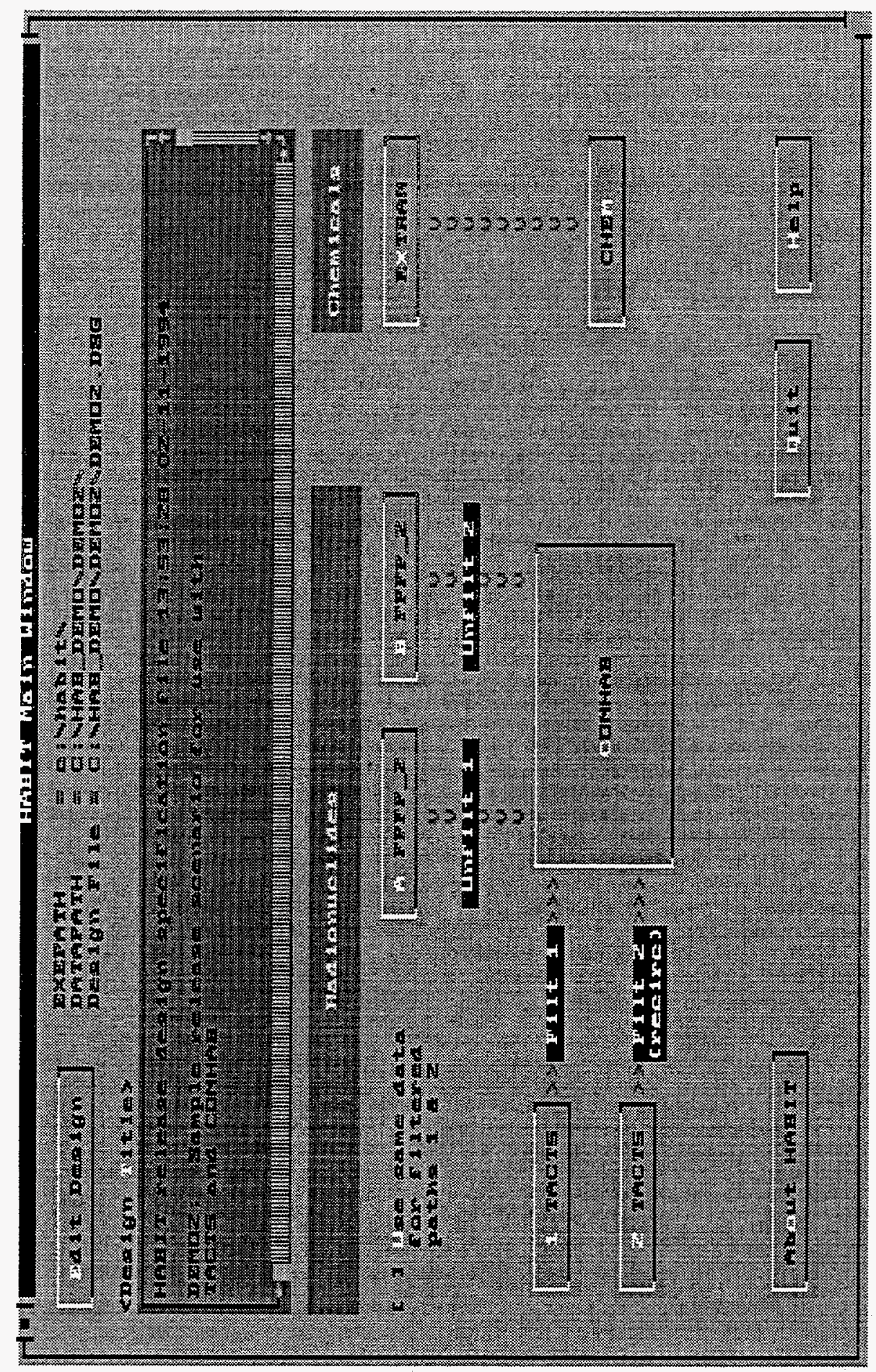

年 


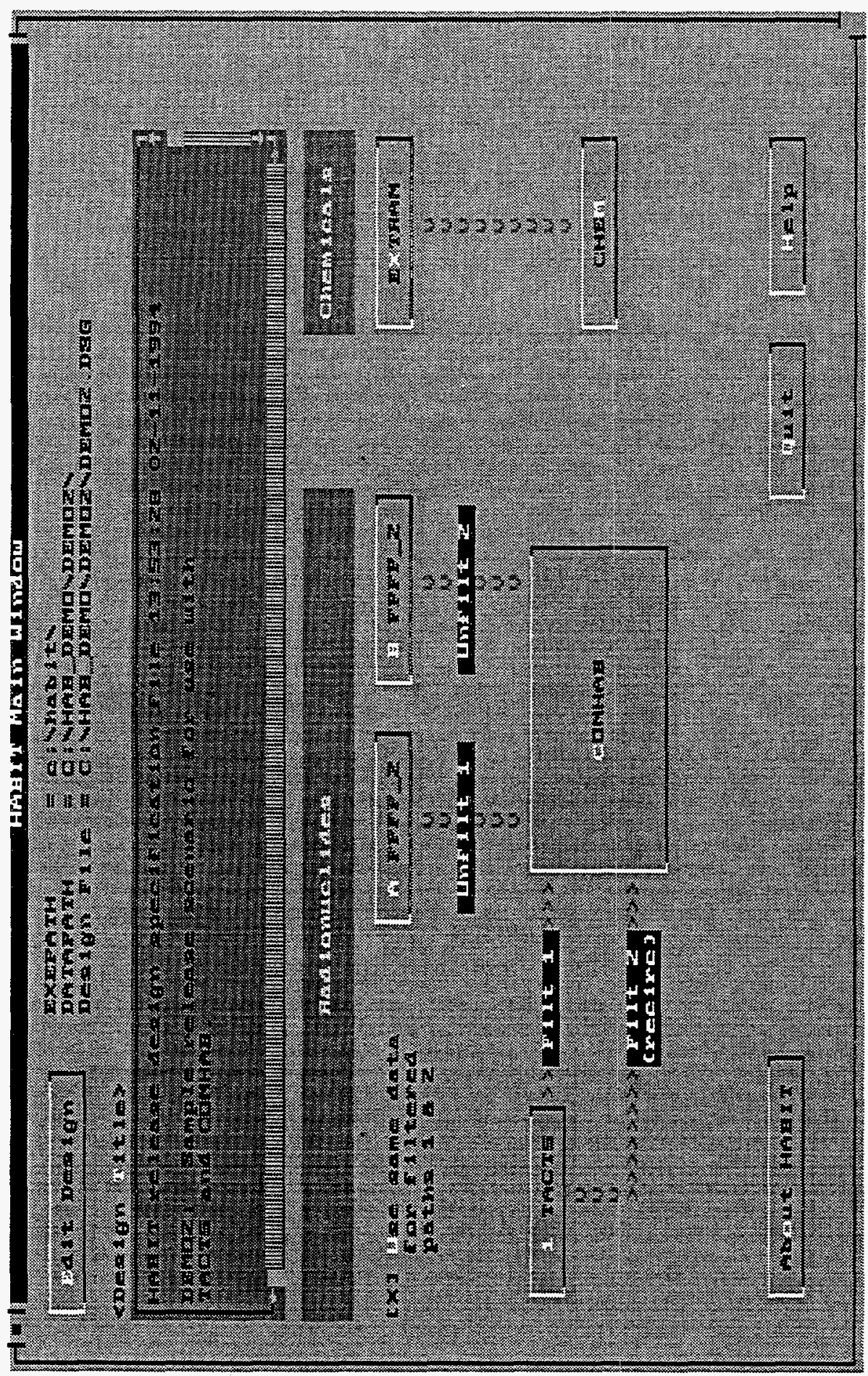




This window (see Figure 5.1) is used to create new designs, use an existing design, copy a design to a new path or ID name, or delete a design. This form appears when the Edit Design button on any of the main data entry windows is pressed.

The buttons on this form are as follows:

The Create New Design button is used when starting a new design. The user is asked to specify the path for the new design and the ID for the design. If the path does not already exist, then the user will be asked if it is okay to create it. The design ID is a string of one to five characters used to identify this particular design.

The Use Existing Design button is used to continue working with an existing design.

The Copy Design button may be used to copy a design to a new path or to a new ID. A designs may be moved to a new path by copying it and then deleting the original. NoTE: The user is cautioned to be sure to check that the copy has been correctly made before deleting the original.

The Delete Design button deletes all files associated with a particular design.

The Done button.

The Help button.

Creating a new design involves creating a file called DataPath \ID---.DSG containing the names of files that may be used for this design. If some of these files aIready exist on the specified path they will be incorporated into the design. This feature may be used by advanced HABIT users to combine parts of various designs into one new design. 


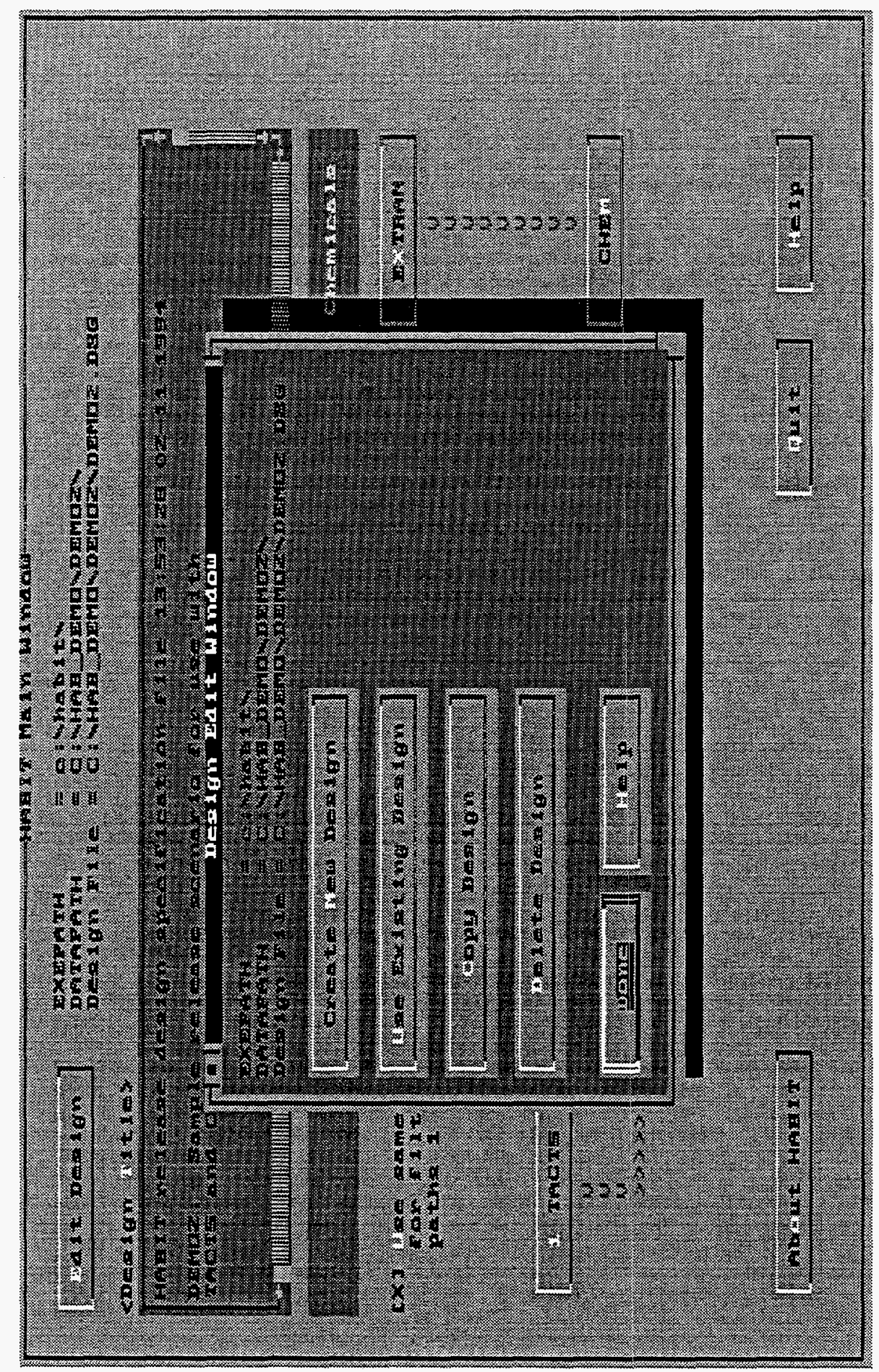

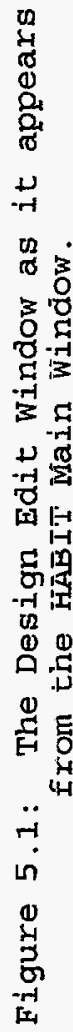


6. EXTRAN :

A COMPUTER CODE FOR ESTIMATING

CONCENTRATIONS OF

TOXIC SUBSTANCES

AT CONTROL ROOM AIR INTAKES.

The EXTRAN code was presented by Ramsdell (1991), which gives a detailed description of EXTRAN. The data input routines have been rewritten, but the variables retain the same meanings as before. Except for the descriptions of the details of data entry, most of the text that follows has been taken directly from Ramsdell (1991) with minor editing changes. It is reproduced here in order that this manual can contain a complete description of the details and use of EXTRAN.

\subsection{General Description of EXTRAN.}

The U. S. Nuclear Regulatory Commission (NRC) staff assesses the potential effects of accidental releases of radioactive, toxic, and explosive substances on the habitability of nuclear facility control rooms. This chapter proposes a procedure for estimating the concentrations at nuclear facility control room air intakes. A computer code called EXTRAN presents the procedure in an easy to use form.

The phrase "toxic substance" will be used in the discussion of EXTRAN, however, it should be noted that many other liquids and gasses satisfy the physical assumptions stated below and can, therefore, be modeled by EXTRAN. One notable case is that many explosive gasses and liquids that produce explosive gasses can be modeled by EXTRAN.

The Pacific Northwest Laboratory (PNL) developed EXTRAN after reviewing procedures used by the NRC for estimating atmospheric transport and diffusion for control room habitability assessments (Murphy and Campe 1974). The review showed that those procedures do not predict variations in the concentrations in building wakes associated with changes in meteorological conditions (Ramsdell 1988). New building-wake diffusion algorithms (Ramsdell 1988, 1990a, 1990b, 1995) predict more than half the observed variability. The EXTRAN code combines procedures for estimating the amount of airborne material, a Gaussian puff model, and the most recent of the building-wake diffusion coefficient algorithms (Ramsdell, 1995).

Given hazardous material in a tank, the user postulates a release scenario, the environmental conditions, and the physical and chemical characteristics of the material. From this information, EXTRAN computes a concentration time history at the control room air intake. Specifically, it estimates concentrations from the time the substance first arrives at the air intake until actions can be taken to protect control room occupants.

Section 6.2.1 presents the equations EXTRAN uses to estimate the transfer of material from the tank to the atmosphere. This transfer may occur in one step, as in release of a gas. Alternatively, it may involve the formation and evaporation of a liquid pool. EXTRAN then calculates transport and diffusion of the material in the atmosphere using a puff dispersion model. The puff model includes a new algorithm (Ramsdell 1995) for estimating diffusion in building wakes. 

Ramsdell (1991) version of the code incorporated revisions based on their comments and suggestions.

The physical setting assumed in the EXTRAN code is simple. It is a tank containing a toxic substance that is directly upwind of a control room air intake. The toxic substance may be gas, a liquefied gas, a volatile liquid, or fine particulate material suspended in a gas. As the simulation begins, the tank fails releasing the toxic material to the environment. When you run EXTRAN, you will be asked to describe the tank failure, the physical relationship between the tank and the intake, the environmental conditions, and the physical characteristics of the material in the tank.

NOTICE: The EXTRAN code does NOT include a model for the rise of a plume from a stack. If the user wants to model a situation in which a toxic substance is released at a temperature significantly warmer than the ambient air or with a significant vertical velocity, the user must first calculate the effective stack height of the plume and use that value for the "Release Height" in EXTRAN. Briggs (1965) gives one method for determining the effective stack height of a buoyant plume. When determining a release height, the user should be aware that the plume may not reach the effective stack height before reaching the control room intake if the release point and the control room intake are close to one another.

Furthermore, the EXTRAN code assumes that the substance released is a "passive scalar", i.e., that it does not significantly effect the stability of the atmosphere. This means that EXTRAN should NOT be used to model a large cryogenic spill, i.e., the spill of a large amount of a substance at a temperature significantly below the ambient air temperature. In particular, results for the release of liquefied gasses must be carefully reviewed. If a Iiquid pool forms that is below the temperature of the ground or the air temperature, then the pool may disturb the atmospheric stability and thus violate the physical assumptions made in EXTRAN.

The work sheet shown in Table 6.3 will help you in using the EXTRAN code. It lists the model options and input. You can record the information that will be needed by the program in the space provided on the work sheet. If you do, you will have organized the information in the order in which the program requests it.

\subsection{The Technical Basis for EXTRAN.}

The EXTRAN computer code is a tool to estimate short-term concentrations at a single location downwind of a release of toxic or radioactive material. The code estimates the amount of material entering the atmosphere and then moves the material downwind as it diffuses. This part of the report describes the technical basis for the EXTRAN code. It includes descriptions of the equations and continuous mass and energy balances used to estimate the source term for the dispersion model. It also includes a description of the dispersion model.

\subsubsection{Source Term Models.}

EXTRAN deals with releases of two types of material: gasses and liquids. 


\title{
6.2.1.A. Gas Tank Bursts and Leaks.
}

\begin{abstract}
All of the parameters for the source term of a gas tank burst or leak are specified by the user. as inputs to EXTRAN.
\end{abstract}

You specify the source term for releases of material stored as a gas when you enter the mass of material in the tank and the leak rate. The mass balance associated with gaseous releases is simple. All of the material leaving the tank enters the atmosphere directly. EXTRAN does not maintain an energy balance for gaseous releases.

If you model a catastrophic failure of a tank containing a gas, all of the gas enters the atmosphere at the time of the failure. Otherwise, you must supply a leak rate as part of the scenario. This leak rate is used to determine the amount of the gas in each puff and the amount remaining in the tank. This information is shown in the first line of each entry in the mass balance file. The remaining lines of the entries in the mass balance file are zero because there is no pool.

\subsubsection{B. Liquid Tank Bursts and Leaks.}

When you model the release of material stored as a liquid, specifying the amount of liquid in the tank and the release rate does not completely determine the source term for atmospheric dispersion. EXTRAN computes the atmospheric source term from information you supply about the liquid's material properties, its storage conditions, and the environment.

The material released from the tank must be partitioned between the atmosphere and the pool. Material in the pool evaporates in response to the vapor pressure of the liquid, and the temperature of the pool is adjusted to maintain an energy balance between the pool and the environment.

EXTRAN assumes that when a tank containing liquefied gasses or volatile liquids fails, the material is distributed between the tank, a pool in the vicinity of the tank, and in a plume in the atmosphere downwind of the tank. A reasonable estimate of the concentrations at a receptor downwind of the tank can only be made if material can be realistically distributed between these locations as a function of time following the release. Mass must not be gained or lost between the release point and the receptor.

The next several sections describe the mass balance for materials that are stored as liquids. Before any of the material can enter a plume, it must be changed to the vapor phase. This change involves energy transfers and transformations. Thus, the mass balance depends on a scenario dependent energy budget. The energy budget will be described in Section 6.2.1.B.b.

\subsubsection{B.a. Mass Balance for Liquefied Gasses and Volatile Liquids.}

As an initial condition, a known mass of the material is assumed to be in a tank. When the tank fails, the material is released to the environment. If the material is a liquefied gas, part of the material leaving the tank flashes at the time of release; the remaining material forms a pool at the base of the tank. The amount of material that flashes can be determined from thermodynamic considerations. If the material is a volatile liquid, all of the material leaving the tank is assumed to gather in a pool at the base of the tank. Material entering the pool is assumed to enter the plume by evaporation. The equations used in EXTRAN to compute the mass of a liquefied gas that flashes and the evaporation of the pool were presented by 


\subsubsection{B.a.I. Flashing}

The mass of liquefied gas that flashes when the material is released can be computed from the mass of the liquid released, its heat capacity and heat of vaporization, and the difference between the boiling point of the liquid and the temperature of the air. The relationship is

$$
m_{f}=m_{r} c_{p}\left(T_{a}-T_{b}\right) / h_{v}
$$

where

$$
\begin{aligned}
& \mathrm{m}_{f}=\text { mass that flashes }(\mathrm{kg}) \\
& \mathrm{m}_{r}=\text { mass released }(\mathrm{kg}) \\
& \mathrm{C}_{\mathrm{p}}=\text { heat capacity }\left(\mathrm{j} / \mathrm{g}^{\circ} \mathrm{C}\right) \\
& \mathrm{T}_{\mathrm{a}}=\text { air temperature }\left({ }^{\circ} \mathrm{C}\right) \\
& \mathrm{T}_{\mathrm{b}}=\text { boiling point }\left({ }^{\circ} \mathrm{C}\right) \\
& \mathrm{h}_{\mathrm{v}}=\text { heat of vaporization }(j / g) .
\end{aligned}
$$

Any liquid that does not flash forms a pool at the base of the tank.

\subsubsection{B.a.II. Liquid Pool}

The liquid pool is assumed to be cylindrical with a volume that is determined by the mass and density of the liquid in the pool. The surface area of the pool is estimated in two ways. It is estimated as a function of time using where

$$
\begin{aligned}
& A_{1}=\pi\left\{r_{0}^{2}+\left[2 t(g V / \pi)^{3 / 2}\right]\right\} \\
& A_{2}=V \cdot 01 m \\
& A^{2}=\operatorname{MIN}\left(A_{1}, A_{2}, A_{\max }\right)
\end{aligned}
$$

$$
\begin{aligned}
& A=\text { area of the pool at time } t\left(\mathrm{~m}^{2}\right) \\
& r_{0}=(\mathrm{V} / \pi)^{1 / 3}=\text { an estimate of initial radius }(\mathrm{m}) \text { made by EXTRAN } \\
& t^{\circ}=\text { time since tank failure (sef) } \\
& \mathrm{g}=\text { gravitational constant }\left(\mathrm{m} / \mathrm{s}^{2}\right) \\
& \mathrm{V}=\text { pool volume }\left(\mathrm{m}^{3}\right) \text {. } \\
& A_{\max }=\text { The maximum pool radius (input by user) }
\end{aligned}
$$

The area $A_{1}$ is based on the spreading of a pool under the force of gravity. The relationship for pool area given by wing (1979) has an additional term that involves the densities of the liquid and air. The value of that term does not deviate significantly from one. Consequently, the term has been dropped from Equation $(6.2 a)$. The area given by $A$, is used to limit on how large the pool can become. The value $A$ ax is used to represent a maximum size of the pool due to a berm or other feature that limits the growth of the pool. If the user enters a value of 0 for $A_{\text {max' }}$ then the program uses $A=M I N\left(A_{1}\right.$, $\left.A_{2}\right)$.

An initial estimate of the pool temperature is made by averaging the temperature of the material being released (boiling point if it flashes) and the temperature of the material already in pool. This temperature is used to estimate the saturation vapor pressure of the material for use in evaporation calculations. 
Evaporation of the pool is driven by the difference between the saturation vapor pressure of the liquid in the pool and the vapor pressure in the atmosphere. For the materials of concern, the vapor pressure in the atmosphere may be conservatively assumed to be zero (wing, 1979, p13) . Therefore, the relationship used to compute the mass of volatile liquid evaporating in an interval dt is

$$
d m_{v}=\left(h_{d} A d t P_{s}\right) /\left(R_{g} p_{p}\right)
$$

where

$$
\begin{aligned}
& \mathrm{h}_{\mathrm{d}}=\text { mass transfer coefficient }(\mathrm{m} / \mathrm{sec}) \\
& \mathrm{P}_{\mathrm{s}}=\text { saturation vapor pressure }(\mathrm{atm}) \\
& \mathrm{R}_{\mathrm{g}}=\text { specific gas constant }\left(\mathrm{atm}-\mathrm{m}^{3} \mathrm{~kg}{ }^{-1} \mathrm{~K}^{-1}\right) \\
& \mathrm{T}_{\mathrm{p}}^{\mathrm{g}}=\text { pool temperature }\left(\mathrm{O}_{\mathrm{K}}\right)
\end{aligned}
$$

The mass transfer coefficient is a function of the radius of the pool, a diffusion coefficient, and the dimensionless Reynolds and Schmidt numbers associated with the air flow across the pool. In EXTRAN the mass transfer coefficient is computed using the relationship given by wing for turbulent flow. That relationship is

$$
h_{\mathrm{d}}=0.037(\mathrm{D} / 2 \mathrm{r}) \cdot \mathrm{Re}^{0.8} \mathrm{Sc}^{1 / 3}
$$

where

$$
\begin{aligned}
& D=\text { molecular diffusion coefficient }\left(\mathrm{m}^{2} / \mathrm{s}\right) \\
& r=\text { pool radius (m) } \\
& \mathrm{Re}=\text { Reynolds Number } \\
& \mathrm{SC}=\text { Schmidt Number. }
\end{aligned}
$$

Molecular diffusion coefficients for many substances have been determined experimentally. They are listed in reference works (e.g., Reid, et al. 1987) . However, there are many substances for which molecular diffusion coefficients are not readily available. If you don't enter a diffusion coefficient as part of the effluent characteristics, EXTRAN will compute one from kinetic theory (Bird, et al. 1960). The equation used in computing the molecular diffusion coefficient is

$$
\left.\left.\mathrm{D}=\frac{0.001853\left[\mathrm{~T}_{\mathrm{T}}\right.}{\mathrm{P}} \frac{3(1 / \mathrm{mw}}{\mathrm{P}} \sigma_{\mathrm{ag}} \Omega_{\mathrm{ag}}+1 / \mathrm{mw}_{\mathrm{g}}\right)\right]^{1 / 2}
$$

where

$$
\begin{aligned}
& T_{x}=\text { reference temperature }(\circ \mathrm{K})=\mathrm{T}_{\mathrm{b}}+273.16 \\
& \mathrm{mw}^{\mathrm{w}}, \mathrm{mw}_{\mathrm{w}}=\text { molecular weights of air and the material in the pool } \\
& \mathrm{P} \mathrm{a}^{\prime} \text { pressure (atmospheres) } \\
& \sigma_{\mathrm{ag}}=\text { effective collision diameter for molecules of air and the gas ( }(\dot{A}) \\
& \Omega \mathrm{ag}=\text { dimensionless function of temperature and intermolecular potential } \\
& \text { figla. }
\end{aligned}
$$

An effective collision diameter for the combination of air and a second gas can be estimated by averaging the collision diameters for air and the gas. The collision diameter for air is 3.617 A. For a liquid, the collision diameter may be estimated from the normal boiling point. EXTRAN estimates the collision diameter for the gas in the tank from

$$
\sigma_{\text {ag }}=1.166 \mathrm{v}^{1 / 3}
$$


where $\mathrm{V}$ is the molecular volume in cubic centimeters per gram-mole (Bird et a1. 1960). The molecular volume is computed from the molecular weight and the liquid density.

The dimensionless function $\Omega$ in Equation (6.5) is estimated using interpolation equations derived from data presented by Bird, et al. (1960 Table B-2). Data in the table indicate that $\Omega_{a g}$ can be estimated adequately as

$$
\Omega_{\mathrm{ag}}=\alpha\left(\mathrm{kT} / \epsilon_{\mathrm{ag}}\right)^{B}
$$

where

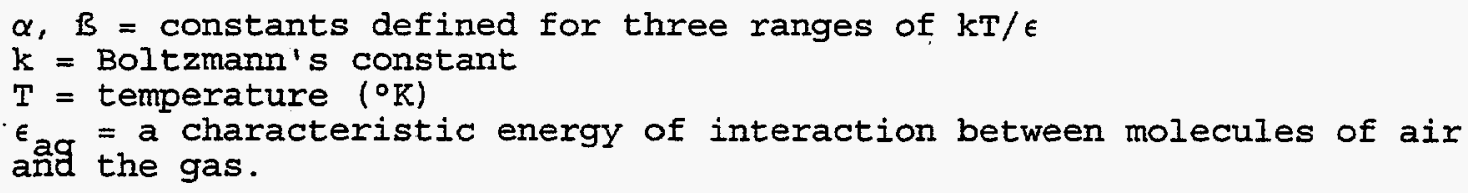

Finally, $\epsilon_{j g}$ is the geometric mean of the characteristic energies for air and the gas. The ratio $\epsilon_{\mathrm{ag}} / \mathrm{k}$ can be estimated from

$$
\epsilon_{\mathrm{ag}} / \mathrm{k}=1.15 \mathrm{~T}_{\mathrm{b}}
$$

where $T_{b}$ is the boiling point of the liquid $\left({ }^{\circ} \mathrm{K}\right)$. For air $\epsilon / \mathrm{k}$ is approximately 97.0 .

The boiling point of liquids depends on atmospheric pressure. Tabled boiling points are generally for a pressure of one atmosphere $(760 \mathrm{~mm} \mathrm{Hg})$. EXTRAN adjusts the boiling point to account for departures from the standard pressure using an integrated form of the clausius-clapeyron equation. The adjusted boiling point is given by

$$
\mathrm{T}_{\mathrm{b}}{ }^{*}=\mathrm{T}_{\mathrm{b}} /\left[1+\mathrm{R}_{\mathrm{g}} \mathrm{T}_{\mathrm{b}} \ln (760 / \mathrm{P}) / \mathrm{h}_{\mathrm{v}}\right]
$$

where

$$
\begin{aligned}
& \mathrm{T}_{\mathrm{b}}^{*}=\text { adjusted boiling point }\left({ }^{\circ} \mathrm{K}\right) \\
& \mathrm{T}_{\mathrm{b}}=\text { boiling point at } 760 \mathrm{~mm} \mathrm{Hg}\left({ }^{\circ} \mathrm{K}\right) \\
& \mathrm{P}^{\mathrm{b}}=\text { atmospheric pressure }(\mathrm{mm} \mathrm{Hg}) .
\end{aligned}
$$

Similarly, the saturation vapor pressure in Equation (6.3) is computed for pool temperature from the boiling point and atmospheric pressure using another form of the integrated Clausius-Clapeyron equation. The equation for saturation vapor pressure is

$$
\mathrm{P}_{\mathrm{s}}=\mathrm{P} \exp \left[\mathrm{h}_{\mathrm{v}}\left(1.0-\mathrm{T}_{\mathrm{b}}{ }^{*} / \mathrm{T}_{\mathrm{p}}\right) / \mathrm{R}_{\mathrm{g}} \mathrm{T}_{\mathrm{b}}{ }^{*}\right]
$$

where all terms are as previously defined.

\subsubsection{B.b. Energy Balance for Liquefied Gasses and Volatile Liquids.}

Several terms in the mass balance just discussed are functions of energy transfer in the environment. This section discusses the components of energy budget of the pool. These components include solar and long-wave radiation, sensible and latent heat exchange with the atmosphere, and heat transfer between the pool and the ground. 


\subsubsection{B.b.I. Solar Radiation.}

The solar radiation balance associated with the liquid pool has two terms, incoming solar radiation and reflection from the surface. The net solar radiation, $\mathrm{R}_{\mathrm{sn}}$, is estimated as

$$
R_{\operatorname{Sn}}=(1-a) R_{S}
$$

where $R_{s}$ is the incoming solar radiation and $a$ is the albedo of the pool. An estimate of the incoming solar radiation is require as part of the model input. Tables 6.1 and 6.2 is section 6.4 provide some guidance on the values of solar radiation observed in the latitude band occupied by the contiguous United States.

The albedo of a pool of any of the materials of concern is unknown. Consequently, a default albedo has been assumed. The default albedo is 0.1 . The assumption of this value is based on albedos reported for sea surfaces (Rosenberg et al. 1983, p. 44). This justification is relatively weak, but the net energy budget is not particularly sensitive to the value assumed. The assumption of a relatively low albedo should be conservative. That is, a low albedo is associated with high absorption of solar radiation which will tend to maximize evaporation.

\subsubsection{B.b.II. Long-Wave Radiation.}

The long-wave radiation balance of the pool is more complicated than the short-wave balance. The long-wave radiation that the pool receives from the atmosphere is a function of the temperature and humidity near the ground and the amount and type of clouds. This is countered by long-wave radiation from the pool, which is a function of the pool temperature.

A number of empirical expressions have been developed to estimate the longwave radiation from the atmosphere. Swinbank (1963) proposed an equation relating the long-wave radiation only to the air temperature based on an analysis of data collected at night. This relationship is reasonable because the humidity at night is also a function of temperature. However, the relationship has been found to systematically overestimate the long-wave radiation during the day. Paltridge (1970) suggests reducing daytime atmospheric long-wave radiation estimates made using Swinbank's relationship by 30 watts $/ \mathrm{m}^{2}$. The same correction to Swinbank's relationship is suggested by Idso (1972). A further correction to Swinbank's relationship to account for the effects of clouds is discussed by Paltridge and Platt (1976). In its simplest form, this correction increases the atmospheric Iong-wave radiation by 6 watts $/ \mathrm{m}^{2}$ for each tenth of the sky covered by clouds. Combining these relationships, the long-wave radiation flux to the pool from the atmosphere is estimated using

$$
\mathrm{R}_{\mathrm{la}}=5.31 \times 10^{-13} \mathrm{~T}_{\mathrm{a}}^{6}+60 \mathrm{cC}
$$

where $R_{l}$ is the long-wave radiation flux from the atmosphere $\left(\right.$ watts $\left./ \mathrm{m}^{2}\right)$ and $c c$ is the cloud cover in tenths. If the solar radiation is greater than 100 watts $/ \mathrm{m}^{2}$, the long-wave radiation flux estimated by Equation (6.12) is reduced by 30 watts $/ \mathrm{m}^{2}$.

The long-wave radiation from the pool is a function only of the pool temperature. It is given by

$$
\mathrm{R}_{\mathrm{lp}}=\sigma \mathrm{T}_{\mathrm{p}}^{4}
$$


where $\sigma$ is the stephan-Boltzmann constant, which has a value of $5.67 \times 10^{-8}$
watts $/\left(\mathrm{m}^{2} \mathrm{O}_{\mathrm{K}}^{-4}\right)$

The net long-wave radiation flux to the pool, $R_{l n}$, is then

$$
R_{\text {In }}=R_{1 a}-R_{1 p}
$$

Estimating this flux requires both the air and pool temperatures. If it is estimated using only the air temperature, the result will be an under estimate of the true flux because the pool temperature will be lower than the air temperature. This will tend to decrease the rate of evaporation and is therefore not conservative.

\subsubsection{B.b.III. Aix Convection.}

The heat flux between the pool and the atmosphere is computed as the product of a heat transfer coefficient and the temperature difference between the pool and the atmosphere. The equation for this flux is

$$
Q_{a}=h_{c}\left(T_{a}-T_{p}\right)
$$

where $Q_{a}$ is the heat $f l u x$ due to convection and $h_{c}$ is the heat transfer coefficlent. The heat transfer coefficient is a function of the thermal conductivity of the air, the air density and viscosity, the heat capacity of the air, a characteristic length, and the wind speed. Assuming that all of these factors are constant except the wind speed, the heat transfer coefficient is computed by

$$
h_{C}=6.690^{0.6}
$$

where $U$ is the wind speed. This relationship is based on an extrapolation by wing (1979) to a wind speed of $1 \mathrm{~m} / \mathrm{sec}$ of values published by Bolz and Tuve (1973).

\subsubsection{B.b.IV. Ground Conduction.}

The heat flux between the ground and the pool is estimated in a manner that is similar to the method used to estimate the heat flux from the air. The flux is proportional to a thermal conductivity and the temperature difference between the ground and the pool. However, unlike the air which passes over the pool, the ground beneath the pool cools as a function of time, which reduces the flux. The equation used to estimate the heat flux from the ground is

$$
Q_{g}=k\left(T_{g}-T_{p}\right) t^{-1 / 2}
$$

where $Q_{g}$ is the $f l u x$ and $k$ is a constant that incorporates the thermal conductIvity, heat capacity and density of the ground. The derivation of Equation (6.17) and $\mathrm{k}$ is discussed by Bird, et al. (1960). Following Wing, when values of the thermal conductivity, heat capacity and density that are typical, of the earth's surface are used, the value of $k$ is approximately 824 $j-\sec ^{172} /\left({ }^{\circ}\right)$. However, $k$ is a strong function of surface type. When the characteristics of specific surface types reported by oke (1978) are used to compute $k$, the values obtained range from 190 for dry peat to 2560 for wet sandy soil. The value for dense concrete is 1780, and bricks and stone have values near 1000. As a result of this diversity, a mid-range value of 1000 has been assumed for $k$ rather than 824 . 


\subsubsection{B.b.V. Latent Heat Transfer.}

As Iiquid evaporates, it carries energy from the pool to atmosphere. The energy lost is equal to the product of the heat of vaporization and the mass evaporated. Thus,

$$
Q_{\mathrm{h}}=\mathrm{h}_{\mathrm{v}} \partial \mathrm{m}_{\mathrm{v}}
$$

where $Q_{h}$ is the latent heat lost. When the pool consists of boiling liquefied gas, the evaporation is determined by the energy entering the pool. As a result, the latent heat loss exactly balances the net energy gained from solar and long-wave radiation, convection and conduction. This balance may be written as

$$
Q_{h}=\left(R_{s n}+R_{l n}+Q_{a}+Q_{g}\right) A d t .
$$

We have assumed that evaporation is determined by vapor pressure.

Consequently, the latent heat loss is not necessarily equal to the input of energy by other mechanisms. If the latent heat loss does not balance the energy gained from other mechanisms, then the temperature of the pool must change as a result of the imbalance. The energy stored or lost by the pool is given by

$$
m_{p} c_{p} d T_{p}=\left(R_{s n}+R_{l n}+Q_{a}+Q_{g}\right) A d t-Q_{h} .
$$

Solving for the temperature change yields

$$
d T_{p}=\left[\left(R_{s n}+R_{l n}+Q_{a}+Q_{g}\right) A d t-Q_{h}\right] / m_{p} c_{p} .
$$

In EXTRAN, the initial pool temperature is assumed to be equal to the boiling point or temperature of the liquid in the tank, whichever is lower. The temperature of the pool is adjusted as a function of time to maintain the energy balance. Equation $(6.21)$ is used to estimate the change in temperature due to evaporation. If the temperature change is negative, the pool

temperature is adjusted accordingly. EXTRAN assumes that the pool remains in the liquid state without freezing. Since freezing reduces the vapor pressure of the material in the pool, this is a conservative assumption.

If the temperature change is positive, EXTRAN makes an initial estimate of the new pool temperature and then compares the estimate with the boiling point of the Iiquid. Should the estimated temperature exceed the boiling point, the pool temperature is set at the boiling point. The energy not used in raising the temperature of the pool to the boiling point is treated as latent heat of evaporation, and mass associated with evaporation is increased.

If the accident scenario involves a leak, the temperature of the pool is also adjusted to account for the difference in temperature between the tank and the pool. In making this adjustment, it is assumed that the liquid entering the pool mixes instantaneously with the liquid already in the pool.

\subsubsection{Transport and Diffusion.}

EXTRAN includes a Gaussian puff dispersion model. This approach was selected because puff models permit more realistic treatment of temporal variations in release terms and concentrations. It is consistent with the Gaussian plume models used by the NRC for other licensing applications and the puff models used for emergency response applications. 


\subsubsection{A. Puff ModeI.}

The derivation of Gaussian plume model starts with a specific solution to the one-dimensional diffusion equation. A three-dimensional puff diffusion model is then produced by superposition of solutions to the one-dimensional equation. If it is assumed that diffusion proceeds independently in the longitudinal, lateral and vertical directions and that the center of the puff is at position $x_{0}, y_{0}, z_{0}$ then, in the absence of boundaries, the concentration at position $x, y, z$ is given by

$$
\begin{aligned}
& C(x, y, z)=Q /\left[(2 \pi)^{3 / 2} \sigma_{x}{ }^{\sigma} y_{z}\right] \\
& \exp \left[-0.5\left(x-x_{0}\right)^{2} / \sigma_{x}^{2}\right] \\
& \exp \left[-0.5\left(y-y_{0}\right)^{2} / \sigma_{y}^{2}\right] \\
& \exp \left[-0.5\left(z-z_{0}\right)^{2} / \sigma_{z}^{2}\right]
\end{aligned}
$$

where

$C(x, y, z)=$ the concentration at $x, y, z$

$Q=$ the mass of material in the puff

$\sigma x, \sigma y, \sigma_{z}=$ diffusion coefficients in the longitudinal,

lateral, and vertical directions.

The diffusion coefficients are characteristic dimensions of the puff. They are functions of the distance (or time) from the release point, the atmospheric stability, and the surface roughness.

Next, a Cartesian coordinate system is defined that has its origin at the ground directly below the release point with the $x$-axis parallel to the wind vector, the $y$-axis directed cross wind, and the $z$-axis vertical. With this definition, the center of the puff can now be allowed to move with the wind. At any moment $t$ following the release, the coordinates of the center of the puff are $x_{0}=U t, y_{0}=0, z_{0}=h$ where $U$ is the wind speed and $h$ is the height
of release. This results in

$$
\begin{aligned}
C(x, y, z)=Q /\left[(2 \pi)^{3 / 2} \sigma_{x} \sigma_{y} \sigma_{z}\right] & \\
& \exp \left[-0.5(x-U t)^{2} / \sigma_{x}^{2}\right] \\
& \exp \left[-0.5\left(y-y_{0}\right)^{2} / \sigma_{y}^{2}\right] \\
& \exp \left[-0.5(z-h)^{2} / \sigma_{z}^{2}\right]
\end{aligned}
$$

The final step in derivation of the plume model is integration of Equation (6.23) from $t=0$ to $t=\infty$. This step makes the plume model a steady-state model. The time delay between the source and the receptor does not appear explicitly in the model, and temporal variations in the source can only be modeled by assuming a sequence of steady-state releases.

The puff model alternative chosen for EXTRAN stops at Equation (6.23). Using the puff model, a plume is approximated by releasing a sequence of puffs at small time intervals. The concentration at a point in the plume is then calculated by summation of the concentrations at the point resulting from all puffs. In essence, the integration that leads to the plume model is replaced by 


$$
C(x, y, z)=\operatorname{Sum}_{i}\left(C_{i}\right)
$$

where $C(x, y, z)$ is the concentration at $x, y, z$ and the $C_{i}$ are the contributions to the total concentration of the individual puffs given by Equation (6.23). It is common to assume that $\sigma_{\mathrm{x}}$ and $\sigma_{\mathrm{y}}$ are equal and to substitute $\sigma_{\mathrm{y}}$ for $\sigma$. Equations (6.23) and (6.24) retain the ability to model the temporal variation in concentrations at an air intake realistically because the concentration will not increase until a puff approaches the receptor, and the values of $Q$ may be varied as a function of time.

The accuracy of the puff approximation can be checked by modeling a steadystate release. It is a function of the distance between puffs. The approximation can be made as accurate as desired by reducing this distance. Ramsdell, et al. (1983) show that if the distance between adjacent puffs is less than $\sigma_{y}$, concentrations estimated by the puff model are within one or two percent of those estimated by a plume model. Puff release rates in EXTRAN are adjusted to maintain this accuracy. (See section 6.2.3.)

Equation (6.23) assumes that the diffusion takes place without the interference of boundaries. That assumption in not tenable for releases at or near ground level. It is common to assume that the ground acts as a reflecting surface. This assumption is incorporated into puff and plume models by assuming an imaginary second source of equal strength located at or below ground at a level equal to the negative of the release height.

Concentrations are then computed by adding the contributions from the real and imaginary sources. Mathematically this is accomplished by replacing the term

$$
\exp \left[-0.5(z-h)^{2} / \sigma_{z}{ }^{2}\right]
$$

in Equation (6.23) with

$$
\exp \left[-0.5(z-h)^{2} / \sigma_{z}^{2}\right]+\exp \left[-0.5(z+h)^{2} / \sigma_{z}^{2}\right]
$$

\subsubsection{B. Source Term.}

Concentrations in the puffs are directly proportional to the mass that is included in the puff. In the EXTRAN code, puffs are released at regular intervals of length dt where dt is determined by the distance between the release point and the air intake, the wind speed, and the atmospheric stability. The mass in a puff released at time $t$ is the mass entering the atmosphere in the period between $t$ and $t+d t$.

If the toxic substance is a liquefied gas and both flashing and evaporation are occurring, two puffs will be released simultaneously. One of these puffs will have the mass of the liquid that has flashed (Equation (6.1)), and the other will have the mass that has evaporated (Equation (6.3)) . Otherwise only one puff will be released, and the mass in the puff will be determined using Equation (6.3).

\subsubsection{Diffusion Coefficients.}

Equation (6.23) shows that the decrease in concentrations in puffs as they move downwind is due only to increases in the magnitudes of the diffusion coefficients. Relationships describing the increase in these coefficients in flat terrain under normal atmospheric conditions are readily available in the literature. The coefficients increase with increasing distance and generally decrease as the atmosphere becomes more stable. 
These standard relationships do not adequately describe the growth of diffusion coefficients in the wakes of structures. The effect of wakes is to increase the rate of diffusion, but the effect is limited to the vicinity of the structure. As result, composite diffusion coefficients that include both normal diffusion and wake effects are used in EXTFAN. These coefficients are computed using

$$
\sigma_{c}=\left(\sigma_{n}^{2}+\sigma_{1}^{2}+\sigma_{2}^{2}\right)^{1 / 2}
$$

where

$$
\begin{aligned}
& \sigma_{c}=\text { the composite diffusion coefficient } \\
& \sigma_{n}^{c}=\text { a normal diffusion coefficient } \\
& \sigma_{1}^{1}=\text { a meander diffusion coefficient. } \\
& \sigma_{2}=\text { a wake diffusion coefficient. }
\end{aligned}
$$

Normal diffusion coefficients are computed with the "Eimutis and Konicek" (1972) relationships used in the NRC PAVAN (Bander 1982) and XOQDOQ (Sagendorf, et al. 1982) codes. In these relationships the diffusion coefficients are functions of distance and atmospheric stability. The diffusion coefficients $\sigma_{1}$ and $\sigma_{2}$ are computed using equations derived by Ramsdell following an analysis of data from building-wake diffusion experiments (Ramsdell 1995). Ramsdell (1988, 1990a,b, 1995) present derivations of the equations.

The puff diffusion equation was derived for point-source releases. The point source equations are reasonable as long as the distance between the release point and the receptor is large. In some EXTRAN applications the point source assumption may lead to unrealistically high concentrations at the source. Consequently, an adjustment is made to the diffusion coefficients to account for the size of the source. The diffusion coefficients are given initial values that result in concentrations at the center of the puff that are no greater than the concentration the pure vapor would have at the atmospheric conditions. These dimensions are related to the density of the vapor and the area of the pool. If a wake is a factor, the adjustment is made to the wake diffusion coefficients. Otherwise, the adjustment is made to the normal coefficients.

\subsubsection{Transport.}

The transport of material is completely defined during model input. Puffs are assumed to move with the wind directly from the release point to the air intake. The time required for material to arrive at the intake is determined by the wind speed and the growth of the puffs. It is somewhat less than the time estimated by $x / 0$ where $x$ is the distance to the intake and $U$ is the wind speed.

\subsubsection{Computational Time Step and Duration of Cormputation.}

The puff release interval used by EXTRAN is given by the following set of rules:

$$
\begin{aligned}
& \text { Let } d t_{\text {puff }}=\sigma_{y} / \mathrm{U} . \\
& \text { If } d t_{\text {puff }}<1 \mathrm{~s} \text {, then use } 1 \mathrm{~s} . \\
& \text { If } 1 \mathrm{~s}<d t_{\text {puff }}<10 \mathrm{~s} \text {, then use the largest even integer }<=d t_{\text {puff }} \text {. }
\end{aligned}
$$


If dt puff $>10 \mathrm{~s}$ and the distance to the intake is less than $1000 \mathrm{~m}$, then tuse $10 \mathrm{~s}$.

If $d t_{\text {puff }}>30 \mathrm{~s}$ and the distance to the intake is greater than $1000 \mathrm{~m}$, then tuse $30 \mathrm{~s}$.

The above rules are designed to ensure that the separation between successive puffs is not more than $2 \sigma_{y^{\prime}}$ and therefore, that the puffs give a good

representation of the plume. They also ensure that the puff spacing is close enough so that EXTRAN can accurately determine the arrival time of the plume.

The time step used by EXTRAN is either 1 s or one half of the puff release interval, whichever is larger.

EXTRAN is designed to determine the maximum concentration reaching the control room within 2 minutes after the arrival of the leading edge of the plume. It is assumed that the control room personnel will be able to take protective measures within that interval.

EXTRAN takes at least 91 time steps. If the time at step 91 is at least 4.5 minutes after the arrival of the plume, then EXTRAN stops. Otherwise EXTRAN runs an additional 90 time steps (180 total time steps). The result of all of these rules is that EXTRAN will run for at least $180 \mathrm{~s}$ after the arrival of the plume and possibly much longer.

The user should examine the time sequence of concentrations found in the file ID---EX.CNX. In most cases the concentrations will either peak and then diminish or will grow towards a maximum and then remain nearly steady until the tank becomes depleted or the liquid pool decreases in area.

\subsection{Starting EXTRAN.}

EXTRAN is started from the HABIT Main window by clicking the EXTRAN button. It may also be started outside of HABIT (i.e., in stand-alone mode) by typing EX at the DOS prompt.

\subsection{The EXTRAN Design form.}

The EXTRAN Design form is shown in Figures 6.1, 6.2, and 6.3. All data input for EXTRAN takes place on this form. The figures for EXTRAN show the way the screens appear when running DEMO1. A label at the top of the form tells the design currently being used. The controls on this form are:

The clear values button is used to clear all values from the EXTRAN input and "start over with a clean sheet of paper."

The Edit Design button may be used to create a new design, to change the design being used, to copy a design or to delete a design. See instructions above on the use of the Design Edit Window.

The Run ritle text box is used for the title of this run.

The Release Type option buttons are clicked to select whether this is a liquid tank burst, a liquid tank leak, a gas tank burst, or a gas tank leak. 
The two liquid options deal with materials stored as liquids and the other two deal with materials stored as gasses. The liquid tank burst and gas tank burst options are used for cases in which the tank fails catastrophically and empties instantaneously. The liquid tank leak and the gas tank leak are used for cases for which the release takes place over some interval. Generally, the catastrophic failure results in a greater maximum concentration than the tank leak mode. But, the tank leak may be the more realistic failure mode. It is possible to approximate a catastrophic failure in the leak failure mode by increasing the magnitude of the leak rate.

The Concentration units option buttons are clicked to select whether the input and output of this run are to be in $\mathrm{g} / \mathrm{m} 3, \mathrm{ppm}$, or $\mathrm{mCi} / \mathrm{m} 3$. Note that The units specified in EXTRAN will also be used in CHEM.

EXTRAN may be used to estimate concentrations of either toxic or radioactive materials. It includes three sets of units for expressing concentrations. The default set of units is grams per cubic meter. It may not be convenient to compare results expressed in these units with standards or to use the results as input to dose models. Therefore, you have the option of two alternate methods of expressing concentrations. EXTRAN can compute concentrations of toxic materials in parts per million, and it can compute concentrations of radioactive materials in millicuries per cubic meter.

The Input Selection option buttons determine whether spill parameters, meteorological parameters, or chemical parameters are displayed on the lower part of this form. Use these buttons to input each of these sets of parameters in succession.

A tip: to enter all data, type values for each of the parameters using the <Enter> key after each entry. When <Enter> is pressed after the last entry of each set, the next set will appear.

The letters $R, O$, and $N$ appear to the left of each parameter. These indicate whether that parameter is required, optional, or not used for the specified type of release. Optional parameters will be used if entered, otherwise default values will be used. A Iist of which parameters are required, optional and not used for each release type is also given in the EXTRAN work sheet.

\section{EXTRAN SpiIl Parameters}

This set of parameters is used to define the physical scenario associated with a potential accident (See Figure 6.1). This input deals with the type of accident, amount of material, where the release occurs in relation to the intake, and how the material enters the atmosphere.

Initial mass

This is the actual amount of material in the tank at the time of failure. The mass should be entered in kilograms if you have selected $\mathrm{g} / \mathrm{m} 3$ or ppm for the concentration units and should be entered in Curies if you have selected $\mathrm{mCi} / \mathrm{m} 3$. In either case, enter the amount in the tank at the time of the failure, not the amount of material that the tank could contain. 


\section{Release rate}

This parameter is only used for liquid tank leak and gas tank leak release types. The release rate will remain constant at the entered value until the tank is empty. When the tank is empty, the release rate will be set to zero.

Release height

For a liquid this is the height of the pool above the ground and not the height of the hole in the tank. For a gas, the release height may be the height of the hole. Release heights greater than zero may result from failure of a tank on a flat roof or loss of the top of a large tank. In the first of these situations the pool might be formed on the roof, and in the second, the pool may be within the original tank. Use ground level near the control room air intake as the reference height.

\section{Storage temperature}

You must enter a temperature for the storage tank for all release types. This temperature determines the expansion of gasses upon release and the rate of evaporation of liquids. Output refers to this temperature as the tank temperature. The tank temperature should be near the air temperature unless the tank is cooled or heated.

Consider factors such as the size of the tank, the exposure of the tank to solar radiation, the insulation of tank, and perhaps the time of day and time of year in estimating the tank temperature. A temperature near the daytime high temperature for the season may be reasonable choice for the tank temperature.

Maximum pool radius

Berms frequently surround large tanks to contain spills. If you specify a liquid tank burst or leak, it may be appropriate to specify a maximum pool radius. If you do not enter a value or enter zero, EXTRAN will assume that a berm does not exist. The liquid will spread without horizontal restriction until the pool depth decreases to $0.01 \mathrm{~m}$.

\section{Distance to intake}

Concentrations in plumes decrease as the distance from the release point increases. When asked for the intake distance, enter the distance between the tank and the control room air intake. If there are structures between the tank and the intake, enter a distance corresponding to the shortest possible path the material could take.

Intake height

The reference height for EXTRAN is ground level near the control room air intake. When asked to enter the intake height, enter the height of the control room air intake above this reference height.

Concentration estimates are sensitive to the difference between the release height and the intake height when distance between the 
tank and intake is small. As the distance increases, this sensitivity decreases. Maximum concentration estimates can be obtained by setting both the release height and intake height to zero. If. the release and intake heights are the same, but are not equal to zero, the concentration will be up to a factor of two lower than when the heights are equal to zero.

\section{Building area}

EXTRAN includes a model to estimate diffusion in the wakes of structures. To use this model, you must enter an estimate of the cross-sectional area of the structure projected on a plane perpendicular to the wind direction. Note that the area requested is the area of the tank or a structure near the tank. It is not necessarily the area of the reactor containment building or of the control room air intake structure. If the area is zero, the enhanced diffusion due to the wake of the structure will be neglected.

vent flow rate $\left(\mathrm{m}^{3} / \mathrm{s}\right)$

The parameter is used only for a gas tank leak. This parameter allows you to model releases through building vents. If the release is through a vent enter the vent flow. Otherwise enter a zero or press RETURN.

The vent flow rate is the volume per unit time of air leaving the vent. The concentration in the vent is therefore the rate of mass release $(\mathrm{kg} / \mathrm{s}$ or $\mathrm{Ci} / \mathrm{s})$ divided by the vent flow rate.

Within BXTRAN the vent flow rate prevents the concentration at the intake from becoming unreasonably large. When the vent flow is greater than zero, concentrations in the environment cannot become larger than the concentration in the vent.

The vent flow is particularly important when the distance between the release point and air intake is small.

\section{EXTRAN Meteorological Parameters}

The next group of parameters provide information on the environmental conditions at the time of the release. These conditions remain constant during the model run (See Figure 6.2). The environmental variables used in EXTRAN enter the calculations in various ways. As a result, no single set of variables can be given the label "worst case." Worst case conditions must be determined by trial and error considering the physical scenario and the material. For example, sometimes worst case conditions involve low wind speeds. However, if the release is in a building wake and involves a volatile liquid, worst case conditions involve high wind speeds.

In selecting a set of conditions for EXTRAN, maintain consistency within the set. For example, a combination of high temperature, high solar radiation, and strongly stable atmospheric conditions leads to larger concentrations than high temperature, high solar radiation and unstable atmospheric conditions. But, the latter combination is realistic, while 
the former is not. Thus, the former set ought not to be used in control room habitability assessments.

\section{wind speed}

The first environmental variable entered is the wind speed. Enter the wind speed at a height of 10 meters above ground level.

The wind speed is used in computing evaporation rates, diffusion and transport. Depending on the specific scenario, increasing the wind speed may increase or decrease the predicted maximum concentration. If the scenario involves evaporation or wake diffusion, increasing the wind speed is likely to result in an increase in the maximum concentration. Otherwise, an increase in wind speed will decrease the concentration.

\section{Atmospheric stability class}

Atmospheric stability classes defined in Regulatory Guide 1.23 are used in EXTRAN.

Atmospheric stability is a factor in determining the dilution of in the plume between the pool and the air intake. If there is not a wake involved, the stability has a significant effect on the maximum concentration. Otherwise, the effect of stability on concentration is small.

\section{Air Temperature}

The ambient air temperature determines if a liquid will flash or not. It is also a factor in the energy balance that determines the rate of evaporation from pools, and it determines the volume occupied by gasses. In general, increasing the air temperature increases the maximum concentration at the air intake following liquid releases and decreases the concentrations following gas releases.

EXTRAN converts the air temperature to kelvin as needed within the code.

\section{Atmospheric Pressure}

Atmospheric pressure is last of the environmental variables used in all scenarios. It is used in adjusting the air density, the boiling point of liquids, and the saturation vapor pressure. Enter the pressure in millimeters of mercury. Pressures in inches, militbars, pounds per square inch, or other units must be converted to millimeters of mercury.

A range of typical atmospheric pressures is from 600 to $800 \mathrm{~mm} \mathrm{Hg}$. This range is larger than would be reasonable for a single location because pressure is a function of elevation. The upper end of the range $(720$ to $800 \mathrm{~mm} \mathrm{Hg})$ is appropriate for sea-level locations. The lower end of the range (600 to $670 \mathrm{~mm} \mathrm{Hg})$ is appropriate for elevations of 5000 to $6000 \mathrm{ft}$. above sea level. 


\section{Solar radiation}

This parameter is used for liquid tank bursts and liquid tank leaks. During the day, solar radiation adds energy to the liquid pool, thereby increasing the evaporation rate.

Enter solar radiation in units of watts/square meter. Enter a zero if you want to simulate a night time release.

During the day, solar radiation is a function of latitude, season and time of day. The data in the following two tables provides some guidance for selection of reasonable solar radiation values. Table 6.1 presents noon-time maximum solar radiation fluxes for the first day of each month based on Weather Bureau Records (Langhaar 1953). The values are for clear days and are rarely exceeded. Table 6.2 presents the average solar radiation for the Hanford Meteorological Station (46 $\left.34^{\prime} \mathrm{N}, 119^{\circ} 36^{\prime} \mathrm{W}\right)$ for $9: 00$ am, noon and 3:00 p.m. for each month. The Hanford Meteorological station is in a semi-arid region in south central Washington State.

Table 6.1 Maximum Noon Sglar Radiation Flux (watts $/ \mathrm{m}^{2}$ )

\begin{tabular}{lrrrr} 
& IATITUDE & & & \\
MONTH & 30 & 35 & 40 & 45 \\
\hline January & 760 & 650 & 540 & 430 \\
February & 850 & 760 & 660 & 550 \\
March & 960 & 900 & 800 & 730 \\
April & 1070 & 1010 & 950 & 880 \\
May & 1140 & 1100 & 1060 & 1010 \\
June & 1150 & 1140 & 1090 & 1060 \\
July & 1150 & 1140 & 1100 & 1070 \\
August & 1140 & 1100 & 1070 & 1030 \\
September & 1100 & 1060 & 990 & 950 \\
October & 990 & 930 & 850 & 770 \\
November & 850 & 770 & 680 & 580 \\
December & 760 & 660 & 550 & 440
\end{tabular}




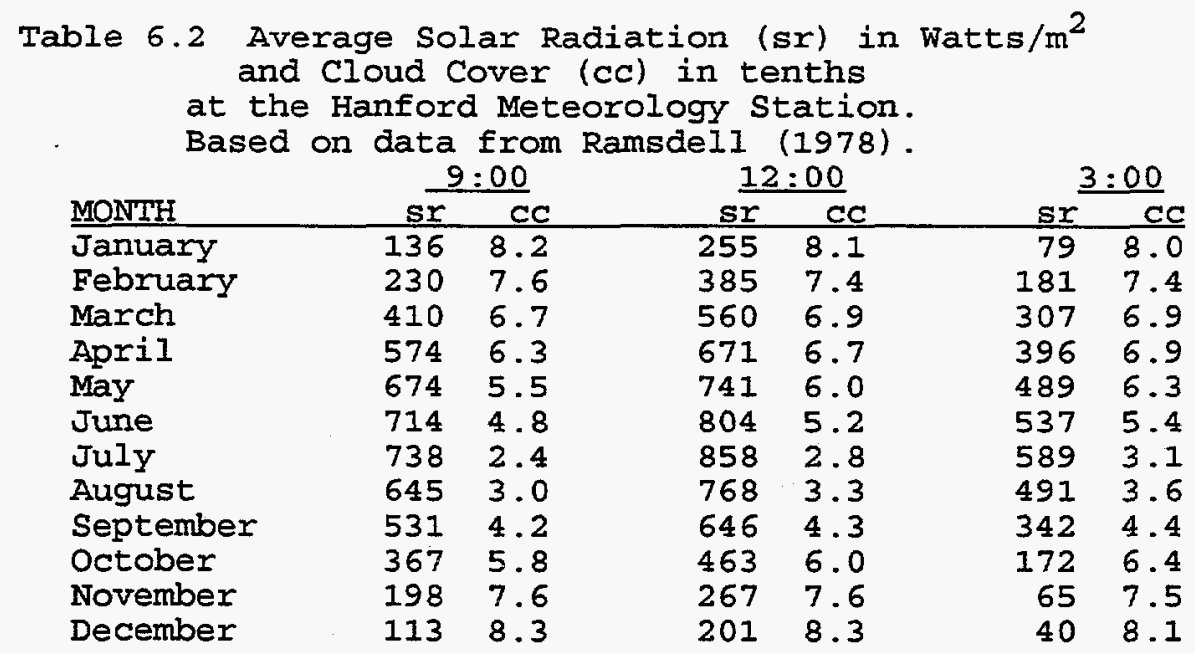

\section{Cloud cover}

This parameter is used for liquid tank bursts and liquid tank leaks. Cloud cover is the fraction of the sky covered by clouds. Enter it in tenths. For example, if clouds cover half sky, the cloud cover would be entered as 5. EXTRAN converts the 5 to 0.5 .

Cloud cover is used in calculating the long-wave radiation from the atmosphere. The program makes an initial estimate of the long-wave radiation from the air temperature. It then refines this estimate using the cloud cover. Cloud cover increases the long-wave radiation flux to the liquid pool. This increase ranges from 0 to 60 watts $/ \mathrm{m}^{2}$.

Table 6.2 presents the average cloud cover, in tenths, for each month at the Hanford Meteorology Station. These values provide some guidance in observed values. You will also want to run the model with 0 and 10 cloud cover.

\section{Ground temperature}

This parameter is used for liquid tank bursts and liquid tank leaks. Along with the pool temperature, it controls the energy flux from the ground to the pool. Increasing the ground temperature increases evaporation.

During the day, the ground temperature in the shade is about the same as the air temperature, while the ground temperature in the open is several degrees warmer than the air temperature. At night the ground temperature is generally be lower than the air temperature.

Enter the ground temperature in degrees Celsius. 


\section{EXTRAN Chemical Parameters}

The last group of parameters defines the physical characteristics of the effluent (See Figure 6.3). These characteristics include the molecular weight, boiling point, heat capacity, heat of vaporization, and specific gravity (or density) of the liquid phase, and the molecular diffusion coefficient.

EXTRAN reads the file CHEMICAL.DAT which contains data for a set of chemicals. One of these chemicals can be chosen using the drop-down list of chemical names. If you choose a chemical from this list, values for all needed parameters will be automatically entered in the value boxes. If you choose "Other" you will need to manually enter values for the needed parameters.

The file CHEMICAI.DAT is an ASCII file that can be readily edited to add chemicals.

Descriptions of these data entries follow:

List of Chemical names. This is a drop-down list containing the names of all of the chemicals for which EXTRAN has data. If you are not familiar with the use of drop down lists, see the section on using them. Using the up and down arrows to scan through this list causes parameters for each chemical to appear in succession. To enter parameters for chemicals that are not on this Iist, select "Other" then enter the needed parameters.

The values in this list are specified in the file CHEMICAL.DAT, which may be edited to add chemicals.

Chemical name

This is an optional parameter which appears in the output.

\section{Molecular weight}

EXTRAN uses the molecular weight in thermodynamic computations for volatile liquids and in estimating the initial volume of gasses.

Boiling point

Enter boiling point temperatures in degrees Celsius. Absolute boiling point temperatures are computed within the program as needed. Boiling point temperatures are a function of pressure. The value entered should be the boiling point at 1 atmosphere pressure $(760 \mathrm{~mm} \mathrm{Hg})$. It will be adjusted to the boiling point at the actual pressure within the program.

\section{Iiquid heat capacity}

The heat capacity determines the fraction of a liquefied gas that flashes on release and the temperature change in pools. It must be in units of joules per gram per degree Celsius. Heat capacities given in calories per gram per degree Celsius may be converted to the required units by multiplying by 4.184 . Within EXTRAN, the heat capacity is a constant, independent of 
temperature and pressure.

Heat of vaporization

The heat of vaporization is the energy used in calculation of the evaporation of liquids. It must be in units of joules per gram. Within EXTRAN, the heat capacity is constant.

\section{Specific gravity}

EXTRAN uses density in calculation of the volume and area of the pool formed by a release of liquids. You may enter either the density, in grams per milliliter, or specific gravity of the liquid. EXTRAN assumes that the density of water is $1 \mathrm{~g} / \mathrm{ml}$. Thus, the density and specific gravity are numerically identical.

Enter the specific gravity of the liquid or the density in grams per milliliter.

\section{Molecular diffusion coeficient}

The rate of evaporation of the pool depends in part on diffusion of the vapor near the pool surface. This diffusion is characterized by a molecular diffusion coefficient. If the substance is a volatile liquid, a diffusion coefficient in units of square centimeters per second must be entered. Molecular diffusion coefficients typically have values near $0.1 \mathrm{~cm}^{2} / \mathrm{sec}$.

If you enter a zero, EXTRAN will estimate the molecular diffusion coefficient from kinetic theory.

The Check Design button. When this button is pressed the design is checked to see whether all required input parameters have been entered. If some required parameters have not been entered a message will be shown and the missing parameters will be highlighted in red until they are entered. Use of this button is optional. The design will automatically be checked whenever the Save Design or Run EXTRAN buttons are clicked.

The Save Design button causes the data to be written to a file. It can be used at any time to save your work. If you change your data and then click either Run EXTRAN or Done you will receive a warning that your data has changed. The message will ask if you want to save your data. If you click Yes, your data will be saved before the run is made or before the program returns to the HABIT main window. If You click No, the file will not be saved and all changes will be lost. If you click Cancel, the Run or Done operation will be aborted.

The Run EXTRAN button. Clicking this button causes EXTRAN computations to be done. Information will be written to the screen about the files being used by EXTRAN and about the progress of the computation. The user can abort the computation by pressing <Ctrl>+C. A message will be written to the screen indicating whether the computation was successfully completed. After the computation is completed the user will be asked to press <Enter> to return to data entry for EXTRAN, press $H$ to return the HABIT main window, or press $X$ to exit HABIT (return to DOS). 
HABIT Manual

NUREG/CR- 6210

EXTRAN

The Done button is used to return to the HABIT main window.

The Help button is used to obtain help. See Using Help for more details.

6.5. Form for EXTRAN Data Entry.

The following page shows a form that may be used to collect data for use in EXTRAN. 
Table 6.3

EXTRAN WORK SHEET

Run ID:

Run Title:

Output options: Concentration Units $\mathrm{g} / \mathrm{m}^{3}$ ppm $\mathrm{mCi} / \mathrm{m}^{3}$ Release Type:

(1) Liquid Tank Burst

(2) Liquid Tank Leak

(3) Gas Tank Burst

(4) Gas Tank Leak

\begin{tabular}{llll}
\multicolumn{3}{c}{ Release } & \multicolumn{2}{l}{ Type } \\
l & 2 & 3 & 4 \\
\hline $\mathrm{R}$ & $\mathrm{R}$ & $\mathrm{R}$ & $\mathrm{R}$ \\
$\mathrm{N}$ & $\mathrm{R}$ & $\mathrm{N}$ & $\mathrm{R}$ \\
$\mathrm{O}$ & $\mathrm{O}$ & $\mathrm{O}$ & $\mathrm{O}$ \\
$\mathrm{R}$ & $\mathrm{R}$ & $\mathrm{R}$ & $\mathrm{R}$ \\
$\mathrm{O}$ & $\mathrm{O}$ & $\mathrm{N}$ & $\mathrm{N}$ \\
$\mathrm{R}$ & $\mathrm{R}$ & $\mathrm{R}$ & $\mathrm{R}$ \\
$\mathrm{O}$ & $\mathrm{O}$ & $\mathrm{O}$ & $\mathrm{O}$ \\
$\mathrm{O}$ & $\mathrm{O}$ & $\mathrm{O}$ & $\mathrm{O}$ \\
$\mathrm{N}$ & $\mathrm{N}$ & $\mathrm{N}$ & $\mathrm{O}$ \\
& & & \\
$\mathrm{R}$ & $\mathrm{R}$ & $\mathrm{R}$ & $\mathrm{R}$ \\
$\mathrm{R}$ & $\mathrm{R}$ & $\mathrm{R}$ & $\mathrm{R}$ \\
$\mathrm{R}$ & $\mathrm{R}$ & $\mathrm{R}$ & $\mathrm{R}$ \\
$\mathrm{R}$ & $\mathrm{R}$ & $\mathrm{R}$ & $\mathrm{R}$ \\
$\mathrm{R}$ & $\mathrm{R}$ & $\mathrm{N}$ & $\mathrm{N}$ \\
$\mathrm{R}$ & $\mathrm{R}$ & $\mathrm{N}$ & $\mathrm{N}$ \\
$\mathrm{R}$ & $\mathrm{R}$ & $\mathrm{N}$ & $\mathrm{N}$ \\
& & & \\
$\mathrm{O}$ & $\mathrm{O}$ & $\mathrm{O}$ & $\mathrm{O}$
\end{tabular}

( $R$ =Required, $0=$ Optional, $N=$ Not used)

Initial Mass ( $\mathrm{kg}$ or $\mathrm{Ci}$ )

Release Rate (kg/sec or $\mathrm{Ci} / \mathrm{sec}$

Release Height (m)

Storage Temp. $\left({ }^{\circ} \mathrm{C}\right)$

Max. Pool Radius (m)

Distance to Intake (m)

Intake Height (m)

Building Area $\left(\mathrm{m}^{2}\right)$

Vent Flow $\left(\mathrm{m}^{3} / \mathrm{sec}\right)$

Wind Speed (m/s)

Atmospheric Stability

Air Temperature $\left({ }^{\circ} \mathrm{C}\right)$

Atm. Pressure (mm Hg\}

Solar Radiation $\left(\mathrm{w} / \mathrm{m}^{2}\right)$

Cloud Cover (tenths)

Ground Temperature $\left({ }^{\circ} \mathrm{C}\right)$

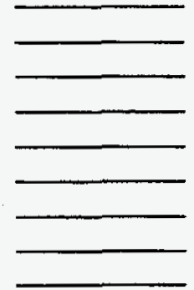

Chemical Name

(EXTRAN will supply the following data if the chemical is listed in CHEMICAL.DAT.)

$\begin{array}{llll}\mathrm{R} & \mathrm{R} & \mathrm{R} & \mathrm{R} \\ \mathrm{R} & \mathrm{R} & \mathrm{N} & \mathrm{N} \\ \mathrm{R} & \mathrm{R} & \mathrm{N} & \mathrm{N} \\ \mathrm{R} & \mathrm{R} & \mathrm{N} & \mathrm{N} \\ \mathrm{R} & \mathrm{R} & \mathrm{N} & \mathrm{N} \\ \mathrm{O} & \mathrm{O} & \mathrm{N} & \mathrm{N}\end{array}$

Molecular Wt. ( $\mathrm{g} / \mathrm{mole})$

Boiling point $\left({ }^{\circ} \mathrm{C}\right)$

Liq. Heat Cap. $\left(j / g-{ }^{\circ} \mathrm{C}\right)$

Heat of Vap. (j/g)

Spec. Gravity

Mol. Diff. Coef. $\left(\mathrm{cm}^{2} / \mathrm{sec}\right)$

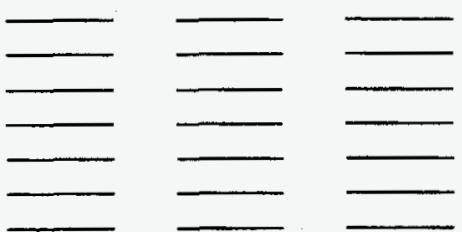

Date:

Time: 


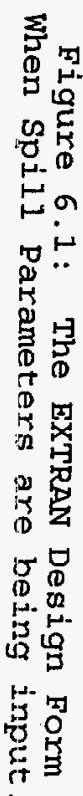

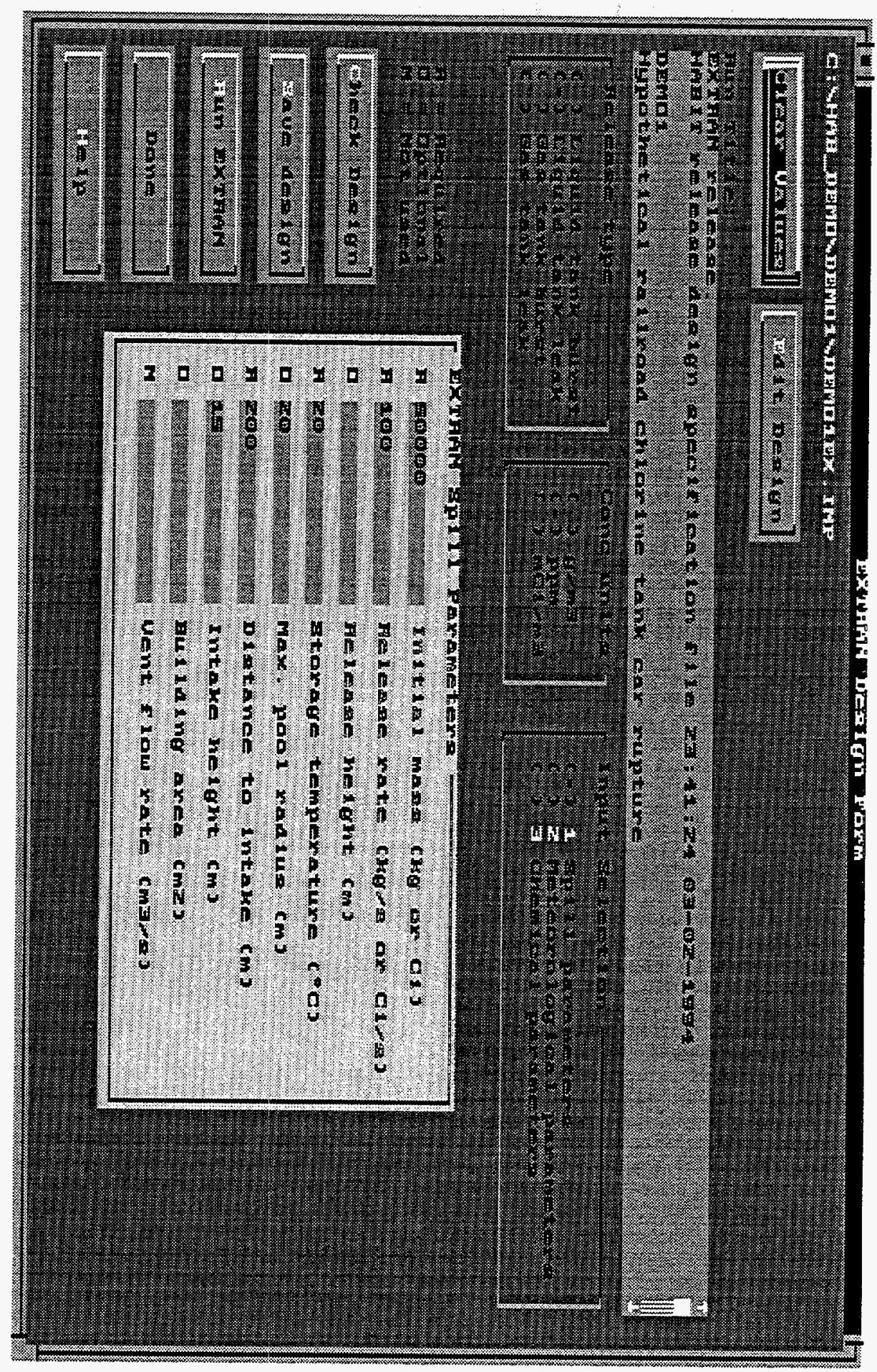




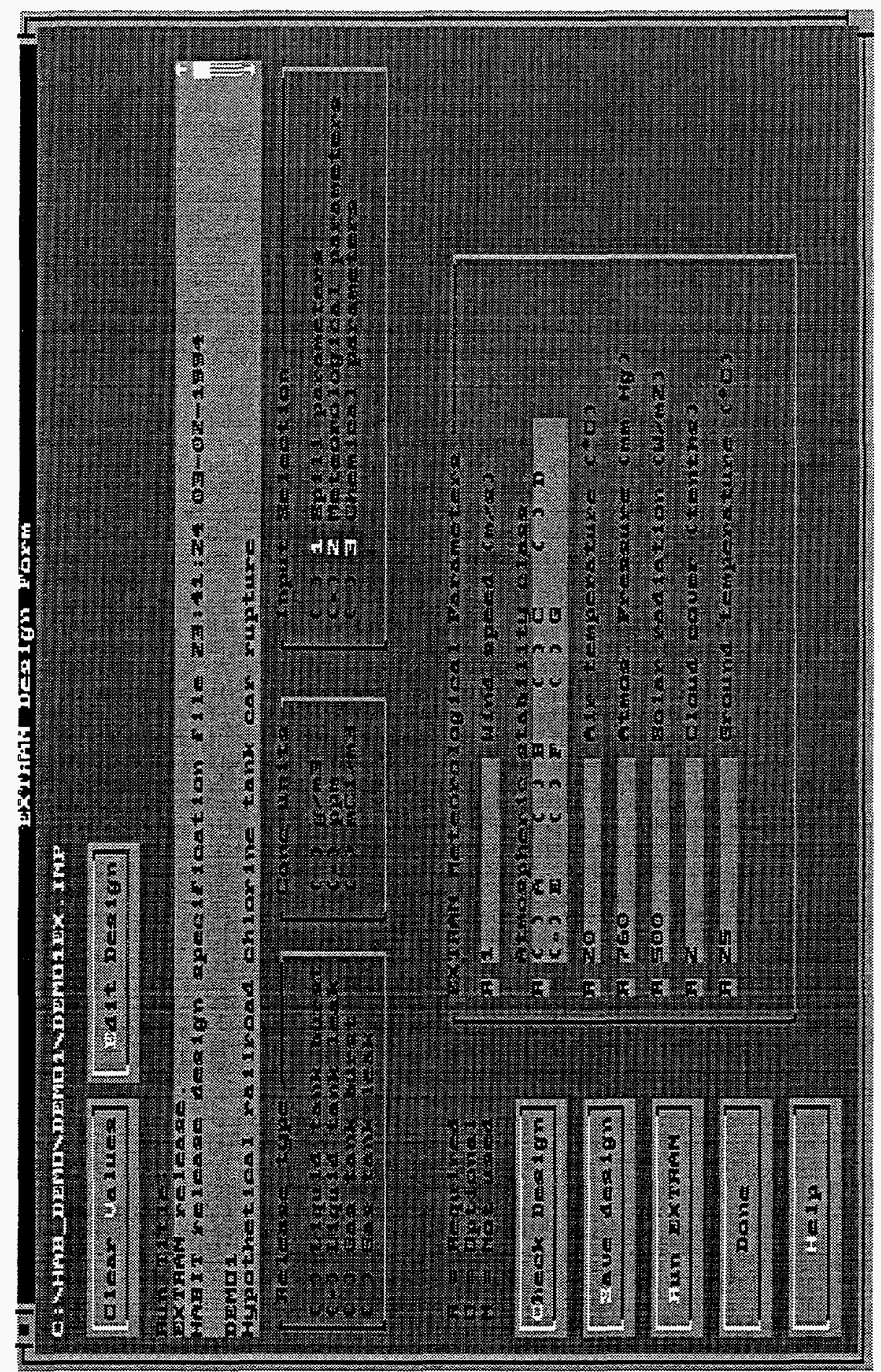

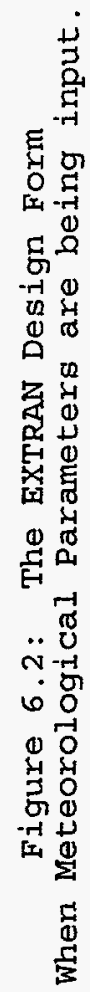




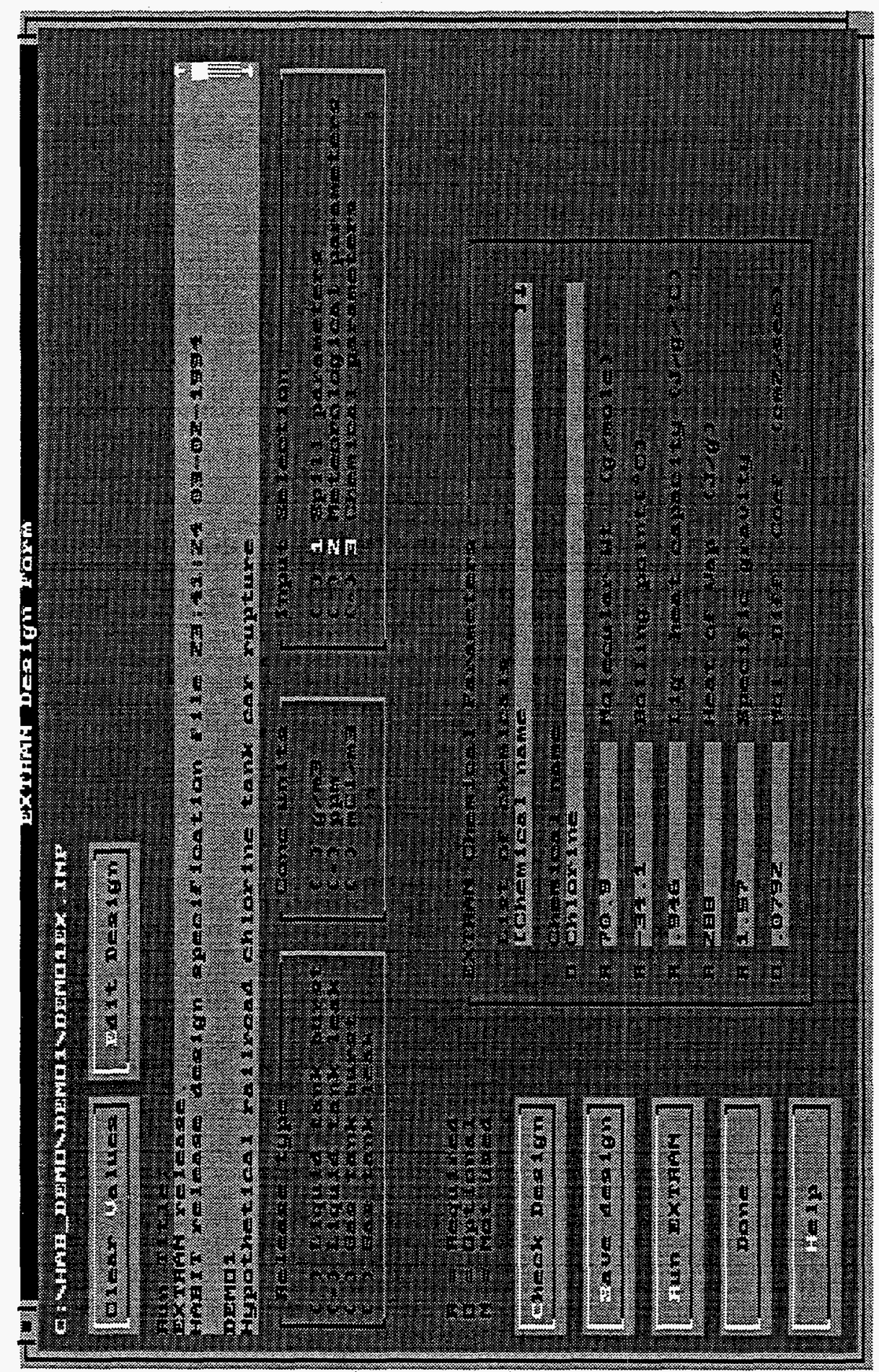

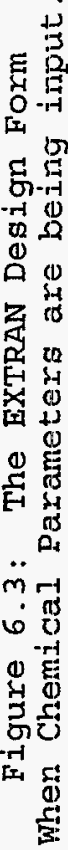




\section{THE CHEM PROGRAM}

\subsection{General Description of CHEM.}

In the CHEM scenario toxic chemicals arrive at the boundary of the control room and are drawn in through any of the four intake paths, namely either of the two filtered or the two unfiltered intakes. These toxic chemicals remain in the control room until they are vented by outflow. It is assumed that toxic chemicals are not effectively removed by filters, settling, or chemical reaction.

\subsection{Theoretical Basis for CHEM.}

The CHEM code is essentially a simplified version of CONHAB (see Gilpin, 1990). All features pertaining to radioactive decay and daughter formation have been removed. Control room filters are assumed to not be effective against the toxic chemicals in question. Concentrations at the control room intakes are computed by EXTRAN. The computations within EXTRAN include the dilution factors, $x / Q$, complete with the effects of building wakes. The only process that needs to be modeled within CHEM is the conservation of chemical mass associated with inflow and outflow. Only one substance need be treated at a time.

The toxic chemical concentration at the control room intakes is obtained from the mean concentration during each time step produced by EXTRAN and read from the file ID---EX.CNX. The concentration is assumed to be the same at all four of the intakes (filterer intake 1, filtered intake 2, unfiltered intake 2, and unfiltered intake 2). Bottled air may flow into the control room. Filters are assumed to be ineffective in removing the chemical. The only mechanism for removal of the chemical from the control room is assumed to be outflow.

Let the concentration at the intakes be denoted by $C_{i n}$ and let $F_{i n}$ be the total flow rate into the control room through the contaminated intakes (i.e., the sum of the flow rates for the four intakes). Further let $F_{\text {clean }}$ be the flow rate of clean bottled air into the control room. The rate clean low out of the control room is then given by

$$
F_{\text {out }}=F_{\text {in }}+F_{\text {clean }} \text {. }
$$

The concentration in the control room is then given by

$$
d C / d t+\left(C F_{\text {out }}-C_{\text {in }} F_{\text {in }}\right) / V=0,
$$

where $\mathrm{V}$ is the control room volume. Given $\mathrm{C}(\mathrm{t})$, the control room concentration at the start of a time step beginning at time $t$, the concentration at the end of the time step is then

$$
C(t+\delta t)=C(t) \exp (-\alpha \delta t)+\left(C_{\text {in }} F_{\text {in }} / \alpha V\right)[1-\exp (-\alpha \delta t)],
$$

where $\alpha=F_{\text {out }} / \mathrm{V}$.

The exposure is just the time integral of the concentration. 


\subsection{Starting CHEM.}

CHEM is started from the HABIT Main window by clicking the CHEM button. It may also be started outside of HABIT (i.e., in stand-alone mode) by typing CH at the DOS prompt. EXTRAN must be run before runining CHEM.

\subsection{The CHEM Main Form.}

The CHEM Main Form is shown in Figure 7.1. The figures for CHEM show the way the screens appear when running DEMO1. A label at the top of the form tells the design currently being used. Note that CHEM and CONHAB share the input file ID--CB.INP. Run title, control room volume, air flow rates, and occupancy factor appear on data entry forms for both CHEM and CONHAB and are used by both of these programs. Filter efficiencies and meteorological parameter values appear only on the CONHAB data entry forms and are used only by that program.

The controls on this form are:

The Clear Values button is used to clear all values from the CHEM input and "start over with a clean sheet of paper."

The Edit Design button may be used to create a new design, to change the design being used, to copy a design or to delete a design. See instructions above on the use of the Design Edit Window.

The Run Title text box is used for the title of this run.

The Control Room volume value box.

The change onits button. The currently selected units are shown with each of the variables. When the change units button is pressed a form appears that can be used to change these units. See section 7.4 .1 on the change units form.

The Enter Control Room Flow Data button causes the CHEM Control Room Flow Parameters Form to appear.

The Save Design button causes the data to be written to a file. It can be used at any time to save your work. If you change your data and then click either Run CHEM or Done you will receive a warning that your data has changed. The message will ask if you want to save your data. If you click Yes, your data will be saved before the run is made or before the program returns to the HABIT main window. If you click No, the file will not be saved and all changes will be lost. If you click Cancel, the Run or Done operation will be aborted.

The Run CHEM button. Clicking this button causes CHEM computations to be done. Information will be written to the screen about the files being used by CHEM and about the progress of the computation. The user can abort the computation by pressing <Ctrl>+C. A message will be written to the screen indicating whether the computation was successfully completed. After the computation is completed the user will be asked to press <Enter> to return to data entry for CHEM, press H to return the HABIT main window, or press $X$ to exit HABIT (return to DOS). 
The Done button is used to return to the HABIT main window.

The Help button is used to obtain help. See Using Help for more details.

\subsubsection{The Change Units Form.}

The change units form, which is used for both CHEM and CONHAB, appears in Figure 7.2. It is accessible by clicking any of the change units buttons which appear throughout CHEM and CONHAB.

The top of this form asks "Do you want numerical values converted?" If the "Yes" option button is selected, all numerical values already in the input will be converted from the old units to the new units. If the "No" option is checked, then the new units will be used for the (unchanged) numerical values.

One of two option buttons is used to choose either meters and meters**3 or feet and feet**3 for distances and volumes respectively.

One of four option buttons is used to select the units for flow rates. Choices available are: $\mathrm{m} 3 / \mathrm{s}, \mathrm{ft} 3 / \mathrm{s}, \mathrm{m} 3 / \mathrm{min}$, and ft $3 / \mathrm{min}$.

\subsubsection{The CHEM Control Room Flow parameters Form.}

This form, which is a schematic representation of the flows into the control room, is seen in Figure 7.3. It is used to enter time steps, control room flow rates and occupancy factors into CHEM. It is identical to the CONHAB Control Room Flow Parameters Form, except that filter efficiencies appear on the CONHAB form but not the CHEM form. Items appearing on this form are:

The step number value box shows the number of the current time step. A number can be typed in this box followed by <Enter> or <Tab> to move to a different time step. Typing a number beyond the last time step defined causes the program to beep and move to the last time step defined.

The step + button is used to move to the next time step.

The step - button is used to move to the previous time step.

The Time drop down list. This list is used to enter new times and to review existing times. To enter a new time, simply type the time in the box at the top of this list and press either <Enter> or <Tab>. The new time will be inserted at the correct place in the sequence of time steps already in the data. To review the data for existing time steps, tab to this and use the up and down arrows to scan through the times. For more information see the section on use of drop-down lists.

The Del Time button. To delete all data for a time step, use the Time drop down list to show the time to be deleted and then press the Del Time button.

The Last Time Defined label shows the step number and time for the last time step that has been defined.

NOTE: CHEM assumes that toxic chemicals flow into the control room through unfiltered path 1, unfiltered path 2, filtered path 1, and 
filtered path 2 (feeds recirculating filter). The chemical concentration is assumed to be the same for all four of the inflow paths and to be given by the values produced by EXTRAN. Flows through all four of these paths are treated identically. You may, if you wish, simply set the flow rate for any ONE of these paths to the total inflow rate and leave the others blank.

The Unfiltered air source 1 value box is used to specify the flow of air into the control room from unfiltered air source 1 .

The Unfiltered air source 2 value box is used to specify the flow of air into the control room from unfiltered air source 2 .

The Bottled air release value box specifies the flow rate of bottled aix into the control room.

The Filtered air source 1 value box is used to specify the flow of air into the control room from filtered air source 1 . Note that filters are assumed to have no effect on the chemical concentrations.

The Filtered air source 2 (feeds recirculating filter) value box is used to specify the flow of air into the control room from filtered air source 2. Note that filters are assumed to have no effect on the chemical concentrations.

A box indicating the position of the recirculating filter.

The control room occupancy Factor value box.

A label indicating the leakage rate of the control room. This shows the total rate at which air must leak from the control room to offset the inflow. It is simply the sum of the inflow rates and is provided for information purposes only.

The Change onits button. The currently selected units are shown with each of the variables. When the change units button is pressed a form appears that can be used to change these units. See the section on the change units form.

The Done button.

The Help button. See Using Help for more details.

\subsection{Forms for CHEM Data Entry.}

The following two pages show forms that may be used to collect data for use in CHEM. 
HABIT Manual

NUREG/CR - 6210

CHEM

CHEM Work Sheet

Plant Parameters

Run ID:

Run Title:

Distance and volume units:

$\mathrm{m}$ and $\mathrm{m}^{3}$

ft and $f t^{3}$

Flow rate units:
$\mathrm{m}^{3} / \mathrm{s}$
$\mathrm{m}^{3} / \mathrm{min}$
$\mathrm{ft}^{3} / \mathrm{s}$ $\mathrm{ft} \mathrm{t}^{3} / \mathrm{min}$

Control Room Volume $\left(\mathrm{m}^{3}\right.$ or $\left.\mathrm{ft}^{3}\right)$ : 
Complete one page for each time step.

Run ID:

Step Number:

Time (hrs):

Flow Parameters:

(Flow units for flows)

Flow from unfiltered source 1

Flow from unfiltered source 2

Bottled air release flow rate

Flow from filtered source $I$

Flow from filtered source 2

occupancy factor (fraction) 


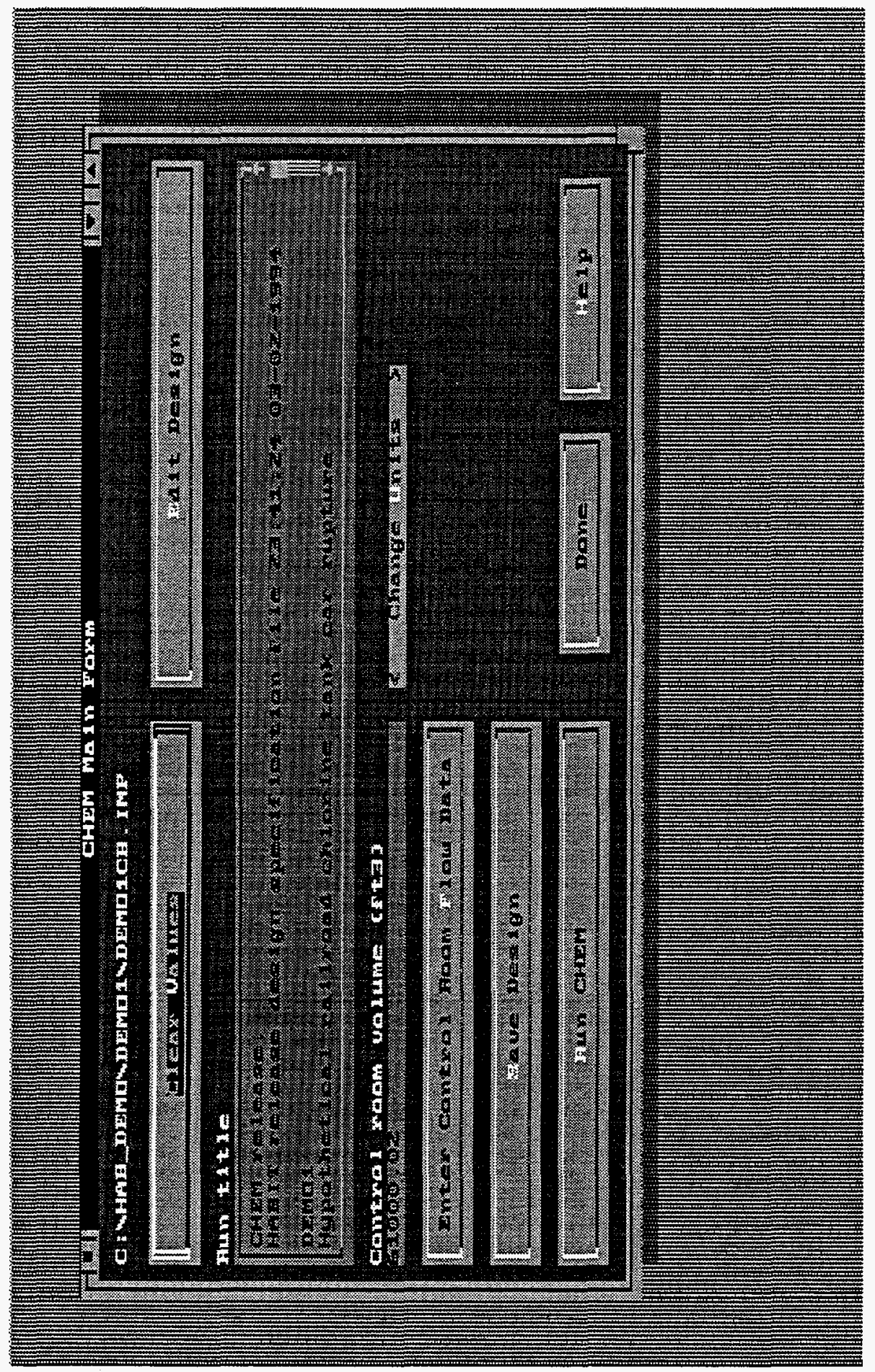

章 


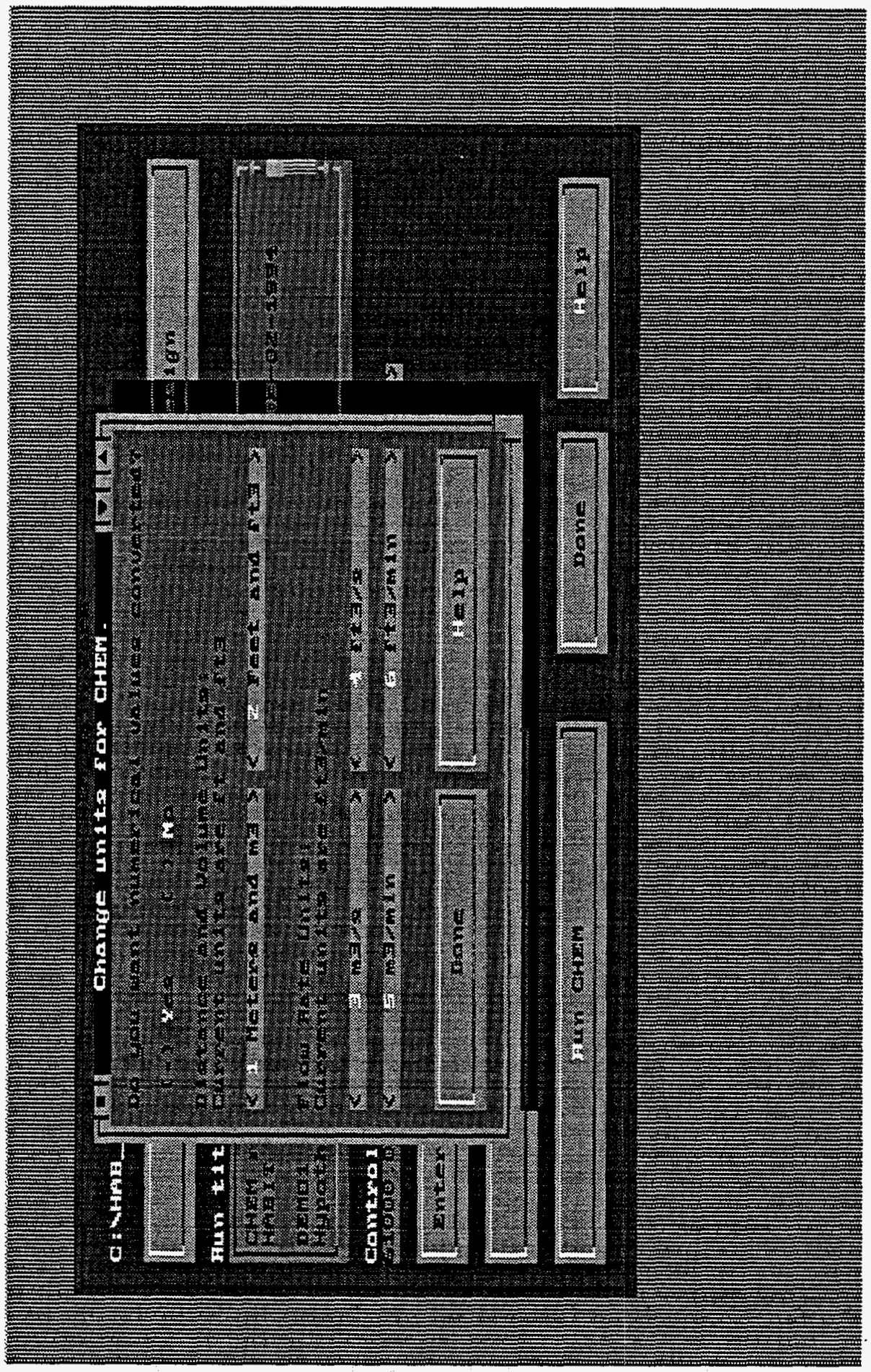

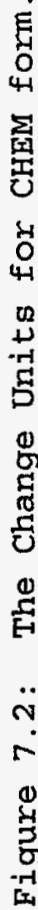




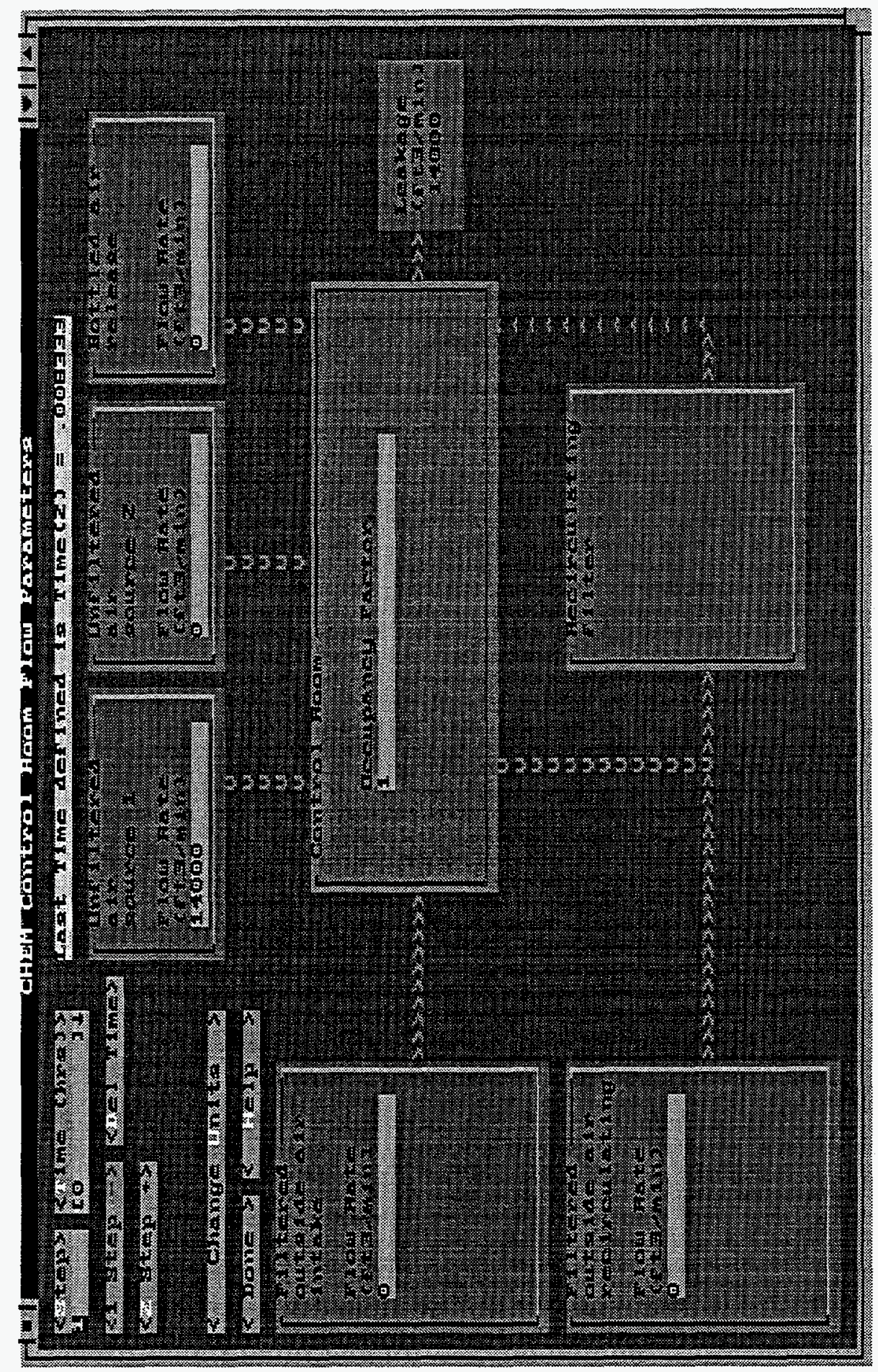

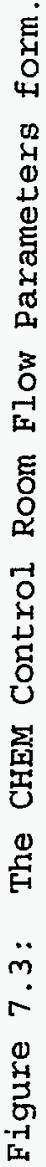



The TACT5 computer code was described by West and Gilpin (1988). As stated in that reference, TACT5 was a revision of an earlier code known as TACT III. The TACT5 code was adapted from TACT III by revising it to run on a personal computer and by removing daughter product formation. The reference for TACT III is G.G. Killough, et al. (1983). The text which follows has been gleaned from a combination of the NUREG/CR's for TACT5 and TACT III, with editing as needed to bring it up to date for the HABIT version of TACT5. The text concerning data entry has been completely rewritten to describe the HABIT version.

\section{I. General Description of TACT5.}

The name TACT5 is an acronym for Transport of Activity. The TACT5 computer code simulates the movement of radioactivity released from a reactor as it migrates through user-defined regions (nodes) of the containment, is immobilized by filters and sprays, and leaks to the outside environment. The user's input data specify a dynamic compartment model which is represented by systems of ordinary differential equations with constant coefficients. The equations are solved using matrix eigenvector methods. A run of the code carries out the integration of these systems of equations over a succession of contiguous time intervals following reactor shutdown, with the interval boundaries corresponding to transitions of system parameter values, which must be constant within each time interval. Outputs are shown for the end of each time interval and include: 1. The level of radioactivity in each node of the containment and released the environment, broken down by nuclide group and by physical form; and 2 . The radiation dose to individuals at the exclusion radius, the boundary of the low population zone, and at the control room air intake. Because of the matrix methods used in TACT5, the accuracy of the results does not depend on the size of the time steps. The HABIT version of TACT5 writes output at sufficiently short intervals to ensure that the output can be used to obtain accurate answers from CONHAB.

The current version of HABIT allows the user to define up to 4 nodes plus the environment.

\subsection{The Technical Basis for TACT5.}

The computational parts of the TACT5 computer code are essentially the same as that reported by west and Gilpin (1988).

Several changes have been made in the TACT5 computational module. The most significant change is adding code so that TACT5 uses time steps small enough to produce output suitable for use by CONHAB. (See Chapter 13.) Changes have been made in the format of the. INP file. The format of the . TAB output file has been improved, and the. CNX file has been added to transfer data to CONHAB. Some changes have also been made to improve the readability of the source code. 
The version of TACT5 in HABIT version 1.0 is capable of treating up to 122 nuclides. The program can handle up to 4 nodes plus the environment and up to 3 receptor points.

TACT5 computes doses to locations in the environment by solving a system of coupled, ordinary differential equations, which are set up by the user through the model description input. The differential equations are set up to track the production and removal of radionuclides through each node by performing a mass balance into and out of each node for each time step.

Sources of production of radionuclides in each node that are considered in TACT5 are instantaneous or continuous release from the reactor core to the node, or from transport from any other node into the node. Sinks of radionuclides from a node include radioactive decay, containment spray removal, filtration, and leakage of radionuclides to other nodes or to the environment.

TACT5 does not directly consider the effects of iodine vapor deposition on the surfaces of the nodes or of particle settling on the floor. It is, however, possible to model these effects. In order to do this, the user must first estimate the removal rate coefficient (per hour) of iodine due to deposition and settling. This value can then be added to removal rate coefficient due to sprays, if any, to form a combined removal rate coefficient. This combined value can be entered in the TACT5 Time Dependent Variables form as the "spray removal rate coefficient" for iodine.

Given this information, a differential equation can easily be constructed relating the rates of production and removal of radionuclides in each node. This system of differential equations is then put into matrix form and solved. This results in a calculation of the amount of a radionuclide (in curies) present in each node and the environment at the end of each time step. The curie contents then are reduced further by the $X / Q$ values at the receptor points in the environment.

The number of curies of each nuclide at the dose evaluation point is multiplied by a dose conversion factor for the specific nuclide and for the organs of interest (and the breathing rate, if appropriate) to yield an organ dose in REM for each time step. The doses resulting from each nuclide are then summed to obtain a dose contribution for the time step and for each organ of interest. The organ doses then are summed over all time steps, resulting in the final calculated dose to each organ of interest for the model.

\subsubsection{The Method of Solution.}

TACT5 uses the data input by the user to determine values for coefficients for a system of coupled first order linear ordinary differential equations. These equations are written in vector-matrix form and are solved using an eigenvector technique. The details of how the coefficients are obtained and the method of solution are given by G. G. Killough, et al. (1983).

\subsubsection{The Unreduced Dose.}

TACT5 produces estimates of doses based on two sets of assumptions. The first of these is labeled in ID---T5A.TAB and ID---T5B.TAB as "Calculated Dose" or as "Multi node containment with ESF". These values are the doses estimated based on the actual plant design and are the values that should be used for most purposes. 
A second set of dose estimates are labeled as "Unreduced Dose" or as "Singie Node Containment with no ESF". These values are based on leakage of the containment directly to the atmosphere at the rate entered as the "Leak Rate of Primary Containment". These values may be interesting to compare to the doses in the first group.

\subsection{Starting TACT5.}

TACT5 is started from the HABIT Main window by clicking the TACT5 button.

TACT5 may also be started outside of HABIT (i.e., in stand-alone mode) by typing $T 5$ at the DOS prompt. If you start TACT5 in this way, you will be working with the Eile names that would be used if you invoked TACT5 by pressing the button on the HABIT main window for TACT5 flow into filtered path I.

\subsection{The TACT5 Release Design Form.}

The TACT5 Release Design Form is shown in Figure 8.1. The figures for TACT5 show the way the screens appear when running DEMO2. A label at the top of the form tells the design currently being used. Generally, the user will enter a design by starting with the Select Nuclides button and working downward through the buttons in succession. The controls on this form are:

The Clear values button is used to clear all values from the TACT5 input and "start over with a clean sheet of paper."

The Edit Design button may be used to create a new design, to change the design being used, to copy a design or to delete a design. See instructions above on the use of the Design Edit Window.

When the Select Nuclides button is pressed, the Nuclide Selection Form appears so that the user can select the nuclides that are used in the TACT'5 computation and the organs for which dose computations are done. (See Nuclide Selection form.) This should be the first step of entering data for TACT5.

NOTE: If any changes have been made to the data, the structure of the data entry programs requires that the TACTS data input file must be saved before selecting nuclides. In this case the program automatically asks if it is okay to save the changed data.

NOTE: TACT5 divides muclides in to five groups: Halogens, Noble Gasses, Solids, Sodiums, and Plutoniums. These groups appear in the form for selecting nuclides, on the form for entering plant data, and on the form for entering time dependent data. However, a group only appears if at least one isotope from that group has been selected. Isotope selections may be changed at any time during data entry or while editing data in preparation for making a new calculation. However, care should be taken when selecting isotopes from a group from which isotopes were not previously selected or when de-selecting all of the isotopes in a group. If the user changes the groups selected, a message will be printed warning the user to check the plant parameters and the time dependent parameters to be sure that they are still correct.

Labels below the Select Nuclides button show the name of the nuclide selection file, the number of groups from which isotopes have been selected, the number of physical forms being used lalways 3 , namely 
elemental, organic, and particulate), the number of organs for which doses will be calculated, and the total number of isotopes selected.

When the Enter Node Design button is clicked the TACT5 Node Design Form will appear. That form allows the user to enter the run title, and the names and volumes for nodes in the design. (See TACT5 Node Design Form). Nodes must be designed before time dependent variables are input. The run title, node names and node volumes may be changed after time dependent variables have been entered, but the number of nodes must NOT be changed.

The Enter Plant Parameters button causes the Plant Parameters Form (see below) to appear. That form is used for entry of the plant power, the elapsed time between reactor shutdown and the start of the release, for core release fractions and plate out factors for each isotopic group, and for isotopic form fractions by group and form.

The Enter Time Data button causes the forms for entering time dependent variables to appear. See "Entering TACT5 Time Dependent Data".

A label below the Enter Time Data button shows the number of time steps that have been defined.

If any changes have been made to the other input data prior to pressing enter Time Dependent Data, the structure of the data entry programs requires that the TACT5 data input file must be saved before entering time dependent data. In this case the program automatically asks if it is okay to save the changed data.

The Time Steps for CONHAB and stand Alone Tjme steps option buttons are used to determine the time steps used by TACT5. When Time Steps for CONHAB is selected, TACT5 will write values to the ID---T5A. CNX or ID-- T5B.CNX file using time steps which are designed to give good computational accuracy when used by CoNHAB. (See chapter 13.) If you intend to use the results of TACTS in CONHAB then you must choose this option to ensure good accuracy of the numbers computed by CONHAB. When Stand Alone Time Steps is selected, TACT5 writes values at only those times that have been entered in the time dependent data table. If you will not be using TACT5 results in CONHAB, you can considerably reduce the TACT5 execution time by selecting this option. The doses computed by TACT5 will be identical regardless of the time step option selected.

The Save Design button causes the data to be written to a file. It can be used at any time to save your work. If you change your data and then click either Run TACT5 or Done, you will receive a warning that your data has changed. The message will ask if you want to save your data. If you click Yes, your data will be saved before the run is made or before the program returns to the HABIT main window. If you click No, the file will not be saved and all changes will be lost. If you click Cancel, the Run or Done operation will be aborted.

The Run TACT5 button. Clicking this button causes TACT5 computations to be done. Information will be written to the screen about the files being used by TACT5 and about the progress of the computation. The user can abort the computation by pressing <Ctrl>+C. A message will be written to the screen indicating whether the computation was successfully completed. After the computation is completed, the user will be asked to press <Enters to return to data entry for TACT5, press 
$\mathrm{H}$ to return the HABIT main window, or press $\mathrm{X}$ to exit HABIT ireturn to DOS).

The Done button is used to return to the HABIT main window.

The Help button is used to obtain help. See Using Help for more details.

\subsection{The TACT5 Nuclide Selection Form.}

The TACT5 Nuclide Selection form is shown in Figure 8.2. When this form appears, it reads the selected nuclide data base file (MIWRFGR.11, MIWRICRP. 30, or MIWRICRP.02, depending on the user's choice. see section 1.10). These files contain nuclide data such as radioactive decay constants and dose conversion factors. If a nuclide selection file already exists, then that file is read to determine which organs and nuclides were selected in that file. The nuclide selection file used by the TACT5 computational routine is similar in format to the nuclide database file (e.g., MLWRFGR.11), but it only contains data for the nuclides and organs that have been selected.

For nearly all applications you will want to skip the controls at the top of this form. You will then begin by using the Organ and Nuclide Selection buttons near the middle of the form.

\section{Use of Buttons for Selecting other Nuclide Data Bases.}

As noted above, you will want to use the default nuclide database for almost all computations. There may, however, be times when you want to make a computation based on an older set of nuclide data. The main reason for doing this would be to compare values with a previous computation based on one of these older sets of values. Unless you have a need to do this type of computation, you may skip the remainder of this section.

Instead of using nuclide data from Federal Guidance Reports 11 and 12, TACT5 can use data from the International Commission on Radiological Protection (ICRP) reports of either 1959 or 1979 . These are contained in the files MLWRICRP.02 and MLWRICRP.30 respectively. TACT5 can also read data from another file of the user's choice, provided that the file is in the proper format.

In order to use another nuclide data base, first click the option button for MUWRFGR.11, MIWRICRP.02, MIWRICRP.30, or other. Then click the Ioad Nuclide Database button. If you have selected "Other" you will be asked for the name of the file to use. The requested data file will be read and its name will appear in the label under the database selection buttons.

The Select Using File button (rarely used) is used to set the organ and nuclide selections to be the same as they were for some other run. organs and nuclides may then be added or removed as desired.

The program can be easily used to make a comparison between doses based on two or more of the nuclide database files. For instance, to compare values based on MIWRFGR. 11 with those based on MLWRICRP.30, first make a run using MLWRFGR.11. Say that we call this run ID11-. Use the Edit Design and Copy Design buttons to copy ID11- to ID30-. Click Select Nuclides from the TACT5 Main Window. On the Nuclide Selection window click MLWRICRP. 30 and then Select Using File using the file IDI1-T5A.NUC. Click Done to close the Select Nuclides window and then Run TACT5 to make the run ID30- based on MLWRICRP.30. 
The Alpha Sort button. (Rarely used.) Normally TACT5 isotopes in each group appear on the Isotope selection form in order of ascending weight. If this button is clicked the isotopes will be sorted to appear in alphabetical order by chemical symbol. A caution, once the nuclides have been sorted in alphabetical order, the only way to go back to ordering by molecular weight is to "Save Nuclide Selections", Load Nuclide Database" "and "Select Using File" based on the selections just saved.

\title{
Making Organ and Nuclide Selections
}

TACT5 can make dose calculations for up to six organs:

\author{
Whole Body \\ Skin \\ Thyroid \\ Lung \\ Bone \\ Liver
}

These organs are selected or de-selected by clicking on each of them. An $X$ appears to the left of all organs currently selected.

TACT5 divides nuclides into five groups. Buttons appear for each of these groups:

\author{
Halogens \\ Noble Gasses \\ Solids \\ Sodiums \\ Plutoniums
}

When one of these buttons is clicked, an "Isotope selection" form appears to allow isotopes within this group to be selected or de-selected. See "Isotope Selection" form. Two numbers are shown on each button to the right of the group name. The first of these numbers is the number of isotopes that have been selected from this group. The second is the total number of isotopes in this group. For example "Solids 3/91" means that 3 isotopes have been selected out of a a total of 91 in the solids group.

A label under these buttons shows the total number of isotopes selected and the total number of isotopes available.

The Save Nuclide Selections button may be used to write your nuclide selections to a file. This may be useful to save your work as protection against loss. If you have made changes and do not use this button, you will be asked when you click the Done button if you want to save your nuclide data.

The Done button is used to indicate that you are done selecting nuclides. If changes have been made in the nuclides selected, you will be asked if it is okay to save the nuclide selection file. If you click "Yes", the file will be saved. If you click "No", the file will not be saved and all changes will be lost. If you click "Cancel" the nuclide selection form will remain visible.

If isotopes have been selected from groups from which isotopes were not previously selected, or if no isotopes are now selected in a group that previously had some selected, then a message will be printed warning you to check input for Plant Design and Time Dependent Variables to be sure that they 
are still correct.

The Help button. See Using Help for more details.

8.6. The Isotope Selection Form.

This form (see Figure 8.3) appears when a group button is pressed on the "Nuclide Selection" form. The name of the group is shown near the top of the form and check boxes are shown for the isotopes in the group. $X$ 's appear in the check boxes of all selected isotopes. Isotopes are selected or deselected by clicking the check boxes.

The All button causes all isotopes to be selected and the None button causes all isotopes to be de-selected.

Once the desired isotopes have been selected, the Done button is used to close the form.

A shortcut: You do not need to use the "Done" button on the "Isotope Selection" form. You may use the mouse to click the button on the "Nuclide Selection" form for another group. You may also click "Save Nuclide Selection", "Done", or "Help" on the Nuclide Selection form without closing the Isotope Selection form.

\subsection{The TACT5 Node Design Form.}

The form can be seen in Figure 8.4 .

The Run Title text box is used to enter the title for this TACT5 run.

The Node Name text boxes are used to enter a name for each node in the design. Use names that briefly describe the nodes. The names you enter here will appear on the forms for entering time dependent data and also in the TACT5 output. The program will not permit you to enter more than eight characters for a node name.

The Node volume value boxes are used to enter the volume of each node. If you have entered a name for a node, you must also enter a value for the volume of that node. Also, if you enter a name or volume for any node, you must enter a volume for every node above it, i.e., for every node having a smaller node number.

A label near the bottom of this form shows the number of nodes that are currently designed. The number of defined nodes is the number of the highest node for which a node name or a node volume has been entered. To reduce the number of nodes defined, delete both the node name and the node volume for the node with the highest node number (See "Deleting Values").

The Done button is used when you are through entering node design data. If you have reduced the number of nodes, you will receive a warning message asking you to confirm that you intended to do so.

The Help button. See Using Help for more details. 
HABIT Manual

NUREG/CR - 6210

TACTS

\subsection{The TACT5 Plant Parameters Form.}

The form can be seen in Figure 8.5 .

The Power value box is used for the rated thermal power output of the reactor in megawatts thermal.

The Elapsed Time value box is used for the elapsed time between reactor shutdown and the start of the accident. If the accident occurs during operation, enter 0 or leave the box blank.

The Core Release Fraction value boxes are used to enter the fraction of the core of each isotopic group that is instantaneously released to the containment.

The Plate Out Factor value boxes are used to enter the fraction of the amount released that instantaneously plates out for each group.

The Isotopic Form value boxes are used to enter the enter the fraction of the isotopic group in each of the three chemical forms used by TACT5, namely elemental, organic, and particulate.

For example, if $95 \%$ of the iodine is released in elemental form, $4 \%$ in organic form and $1 \frac{\circ}{6}$ in particulate form then the values $0.95,0.04$, and 0.01 would be entered here.

The Done button is used to indicate that all plant parameters have been entered.

The Help button. See Using Help for more details.

\subsection{Entering Time Dependent Data.}

The TACT5 time dependent variables include parameters that may change with time. These describe how radioactivity is released to the nodes, the rates of exchange of air between the nodes, the removal of radioactivity from the system (e.g., by filters and sprays), leakage of radioactivity to the outside environment, and parameters related to individual exposure (i.e., breathing rates and dilution factors, $\mathrm{X} / \mathrm{Q}$ ).

From the "TACT5 Release Design" window, click the "Enter Time Dependent Data" button. Isotopes selections, plant parameters, and node parameters must be entered before entering time dependent data. Isotopes selections, plant parameters, and node parameters may be changed after entering time dependent data; however, you should NOT change the groups from which isotopes have been selected, and you should NOT change the number of nodes in the design.

Time dependent data is entered into TACT5 with the help of two forms. The "TACT5 Time Dependent Variables" form appears first and is used to choose which variables you want to enter. This form controls the behavior of the "TACT5 Time Dependent Variable Input Form".

The "TACT5 Time Dependent Variable Input Form" is used to actually enter the data and is similar to a spreadsheet. At any given time, one variable appears on this form. Buttons on this form allow you to move through the variables. 
This form (see Figure 8.6) is used to choose which TACT5 time dependent variables you will enter. This form has a check box for each TACT5 time dependent variable. X's appear next to each of the variables that will be input.

If no time dependent variables have been entered, the check boxes for all of the variables will be marked. Initial Activity will be marked for input as a fraction and continuous activity release will be marked for input as fraction per hour. Neither will be marked for input independent of isotopic group.

If time dependent data has been entered for this design, then boxes will be marked only for those variables that have been entered.

Click on these boxes to toggle the X's on or off. (See "Use of Check Boxes").

Note that the X's on this form control the functioning of the TACT5 Time Dependent Variable Input Form, but do not directly alter the database. If you remove an $X$ from one of these boxes, that variable will not appear as you step through the variables on the TACT5 Time Dependent Variable Input Form unless the "Review All" option is checked. However, any existing values of the variable are NOT deleted from the database.

The TACT5 time dependent variables are:

\section{Initial Activity Distribution}

This is used to tell how the activity is distributed for an instantaneous release at the start of the accident.

Depending on which of two option boxes is checked, the initial activity distribution can be specified as the Fraction of the core that is distributed to each of the nodes or the number of Curies.

When the Independent of Isotopic Group check box is marked with an $x$, then the initial activity will be assumed to be distributed in the same way for all of the isotopic groups. If this box is not marked, values of initial activity distribution will be requested for each isotopic group.

\section{Continuous Activity Release}

This is used to describe a release of activity that takes place over a period of time rather than instantaneously.

Depending on which of two option boxes is checked, the continuous activity release can be specified as the Fraction per hour of the core that is released into each of the nodes or as the number of Curies per hour released into each of the nodes.

When the Independent of Isotopic Group check box is marked with an $x$, then the continuous activity release will be assumed to be distributed in the same way for all of the isotopic groups. If this box is not marked, values of continuous activity release will be requested for each isotopic group. 
HABIT Manual

NUREG/CR - 6210

TACT5

\section{Spray Removal Rate Coefficients}

This allows the user to model the effect of containment sprays on nuclide concentrations by entering removal rate coefficients in units of $1 /$ hour.

As explained above, settling and deposition of iodine vapor may be modeled in TACT5 by adding the removal rate ( 1 /hour) for settling and deposition to the removal rate for sprays and entering the sum here. In this case, it would be more correct to refer to this variabie as the "total removal rate coefficient" rather than the "spray removal rate coefficient".

Spray removal coefficients may not be entered for noble gasses because they not be removed by sprays.

\section{Filtered Transfer Rates Between Nodes and Filter Efficiencies}

The filtered transfer rates are the rates of air exchange from any node to the environment and from any node to any other node. These exchange rates are expressed in cubic feet per minute (CFM). Flows driven by fans would be modeled using this parameter.

When this parameter is selected, values may also be entered for the filter efficiencies associated with each of the transfers. A value of the filter efficiency may be entered for each form (elemental, organic and particulate) of each isotopic group except noble gasses. If there are no filters or if a filter is not effective for a particular form of an isotopic group, then the filter efficiency may be left blank.

\section{Unfiltered Transfer Rates Between Nodes}

This parameter is used for transfer that is not subject to filters. It is similar to the filtered transfer except that the transfer rates entered for this option are generally associated with convective flows and are in units of percent per day.

A general rule for convective flow is that whenever two nodal volumes are in contact with each other, twice the smaller nodal volume is exchanged between the two nodes per hour. (See SRP section 6.5.2.)

Note that the total transfex between nodes is the sum of the filtered transfer and the unfiltered transfer. It is NOT correct to specify a transfer rate using the filtered transfer rate in CFM and then include the same rate again as an unfiltered transfer rate in percent per day. That would have the effect of doubling the transfer rate.

\section{Dose Parameters}

The travel time to receptor, leak rate of primary containment, dilution factor $(\mathrm{X} / \mathrm{Q})$, and breathing rate are collectively referred to as dose parameters. These are used in computing the doses at the receptor points.

TACT5 currently uses up to three receptor points. These are the exclusion area boundary (EAB), the low population zone (LPZ) boundary, and an other receptor of the user's choice.

It is useful to use the "other" receptor as the control room intake. Then the dose produced by TACT5 may be used in the Murphy-Campe (reduction factor) method to obtain an estimate of the dose inside the control room. This may 
provide a useful number for comparison with the control room dose estimate produced by CONHAB. One may also compare the TACT5 estimate of the dose outside the control room with the CONHAB estimate of the dose inside the control room to obtain a reduction factor for the control room design. An example of this sort of comparison is given in the discussion of DEMO2 in Chapter 11 .

NOTE: CONHAB uses the concentrations released to the outside atmosphere as computed by TACT5 and written in the file ID--T5A.CNX. CONHAB then uses its own values for travel time and $X / Q$ to get the concentration at the control room air intake for subsequent CONHAB calculations. The travel times, leak rate of primary containment, $X / Q$ values, and breathing rates entered in TACT5 do NOT have any effect on dose estimates produced by CONHAB.

\section{Travel Time to Receptor Points}

This is the elapsed time between when activity is released to the environment and when it arrives at each of the receptor points. This parameter is used to account for radioactive decay en route to the receptor.

\section{Leak Rate of Primary Containment}

The leak rate of the primary containment is entered here in units of percent per day. This parameter is used only in the computation of "unreduced dose". See section 8.2.2 for an explanation of "unreduced doses". It does not effect any of the "calculated dose" values and does not effect conHAB dose estimates.

\section{Dilution Factor, $(\mathrm{x} / \mathrm{Q})$}

Dilution factors $(X / Q)$ in units of seconds per cubic meter must be entered for each receptor point for which a dose estimate is desired.

\section{Breathing Rate}

A breathing rate in cubic meters per second must be entered for each receptor point for which a dose estimate is desired.

Once you have marked the check boxes you are ready to begin entering the time dependent values. Click the Enter values button to make the TACT5 Time Dependent Variable Input Form appear.

The Done button can be pressed to indicate that you are finished with input of time dependent variables. If you have made any changes to the data, you will be asked if it is okay to save the changes. The forms for inputting time dependent variables will then disappear and control will return to the TACT5 Release Design form.

The Help button. See Using Help for more details. 8.9.2. The TACT5 Time Dependent Variable Input Form.

This form (see Figure 8.7) is used to actually enter the TACT5 time dependent data. The top of this form tells which time dependent parameter is displayed. One parameter is displayed at a time. The middle of the form is like a spreadsheet for data entry. The bottom of the form has several command buttons used to change which variable is displayed, insert and delete times, and to indicate when input is complete.

As you use the buttons at the bottom of this form to move through the input 
variables, the name of the variable currently displayed will be highlighted on the variable selection form as an aid to keeping track of where you are in the data entry process.

\subsubsection{A. Information at the Top of the TACT5 Time Dependent Variable Input} Form.

The top of the form shows the variable being displayed and the units for that variable. Additional items, which may be shown depending on what is needed, are the isotopic group, the form, and the node number and name from which flow is going.

\subsubsection{B. The Data Table at the Middle of the TACT5 Time Dependent Variable} Input Form.

This is a spreadsheet-like display of values for use in entering, reviewing and editing values. Time steps are displayed down the left side of the table and values are displayed in the remainder.

HINT: The simplest way to enter data is to use <Tab>, the arrow keys, or the mouse to place the cursor in a cell of the table, type a value and then press <Enters. The cursor will then move to the next cell of the table. Then proceed to type values using the <Enter> key after each. After all values for a given variable have been entered, use the buttons at the bottom of the form to display the next variable.

Time steps can be entered by simply typing the value for the time in the blank cell at the bottom of the time column. The new time will automatically be inserted in the correct place amid any existing time steps.

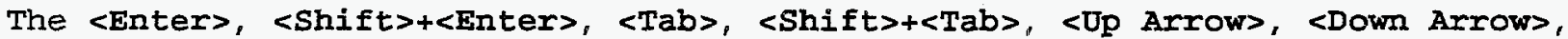
<Page Up>, and <Page down> keys can all be used to move around the data table.

NOTE: TACT5 assumes that values remain the same until a change is indicated by entering a new value. You, therefore, only need to enter values for a parameter at those times when the value of that parameter changes.

8.9.2.C. The Buttons at the Bottom of the TACT5 Time Dependent Variable Input Form.

These buttons are used to change which variable is being displayed on the form, to insert and delete times, and to indicate when you are done with entry of time dependent variables.

The Review All check box near the lower right corner of the form has an important function in determining which variables are displayed.

During normal data entry the Review All check box is NOT checked. In this case the program will display only those variables that have been selected by checking boxes on the TACT5 Time Dependent Variables form.

When the Review AlI check box is checked, the program will display all of the variables for which at least one value has been entered. This is useful when reviewing a design to confirm that all values have been correctly entered. When Review All is checked, the selections made on the TACT5 Time Dependent variables form have no effect on which variables are displayed.

The First, Previous, Next, and last buttons change which variable is displayed. 
The Choose Input button switches to the TACT5 Time Dependent Variables form so that the variables selected for input can be modified. Use the "Enter Values" button on the TACT5 Time Dependent Variables form to resume entering values.

The Delete Time button can be used to either delete a time step for all

variables or to delete the values of the current variable for a time step. To use this button, use <Tabs or the mouse to place the cursor on the time box or one of the values boxes for the time step to be deleted. Then click Delete Time. A window will then appear asking you to click YES to delete values for all values for all variables, to click No to delete values from this screen only, or to click Cancel. If Yes is pressed the time step itself will also be deleted.

The Insert Time button. New times may be entered into the data by using this button or by simply typing the new time into the empty time value box at the bottom of the time column. In either case, the new time will be inserted at the correct location in the sequence of existing times.

The Delete All button causes all time steps and time dependent data to be deleted from the data base. To guard against accidental loss of data, a warning message is displayed before proceeding.

The Save Design button can be used at any time to save your changes. It is a good idea to periodically save your changes to avoid losing your work, however, when you click the done button, you will be asked whether it is okay to save your changes.

The Done button is used after all time dependent variables have been entered to return to the TACT5 Design form.

The Help button. See Using Help for more details.

\subsection{Forms for TACT5 Data Entry.}

TACT5 does not readily lend itself to the use of standardized forms for data entry. It is suggested that you use the following steps when gathering data for use by TACT5.

Make a diagram showing the nodes that are needed for this design. You will need to supply a name and volume for each node. Determine values for the time dependent parameters. Determine which nodes receive an initial activity and which nodes receive continuous activity release. Sketch the various flows which take place between nodes and from nodes to the environment. Determine whether these flows fit the descriptions given above for filtered (forced) flows or unfiltered (convective) flows and determine flow rates. Determine any needed filter efficiencies. Determine travel times to the receptor points, X/Q values, and breathing rates for the receptor points, as needed. 


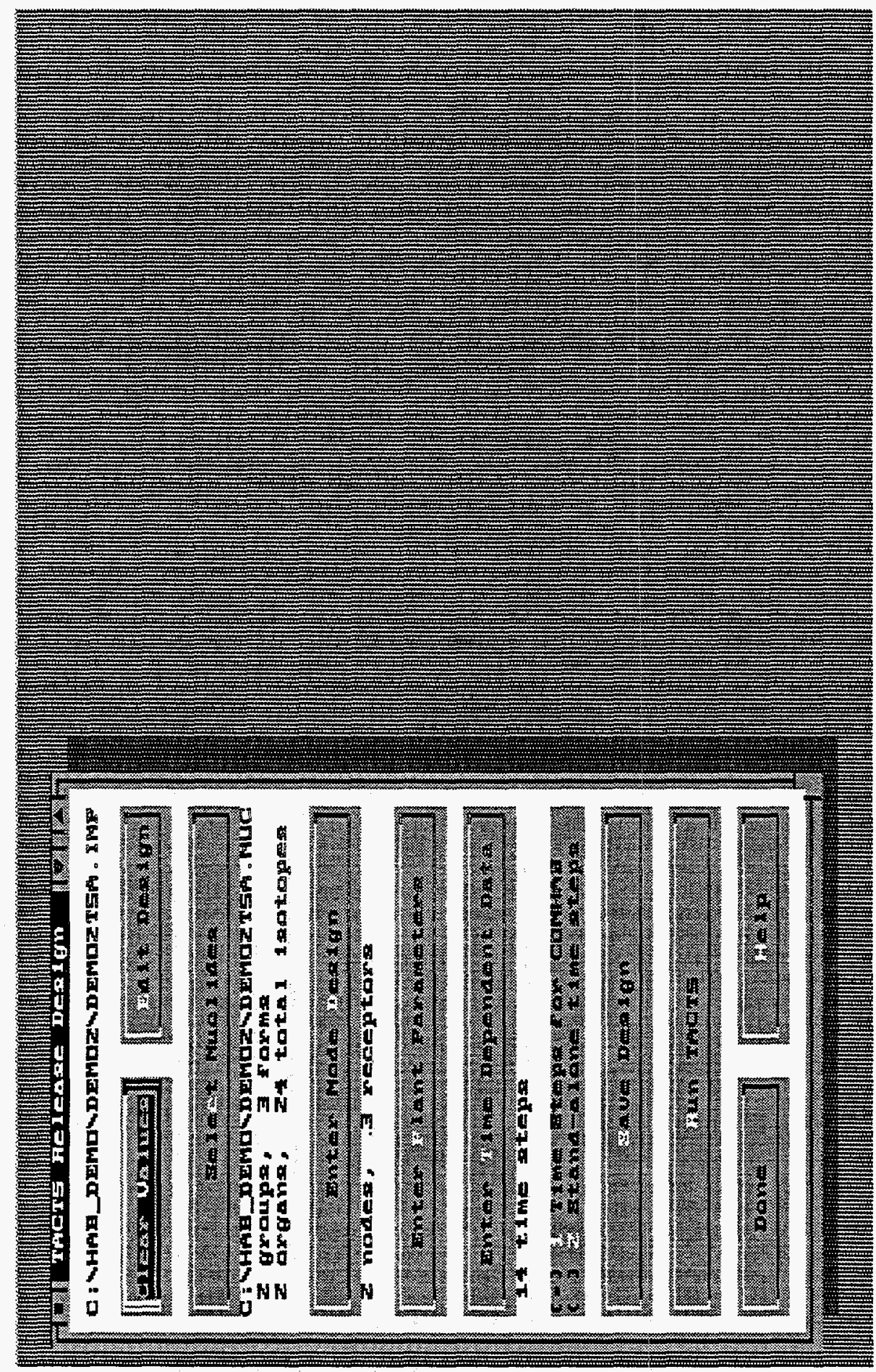

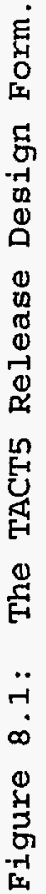




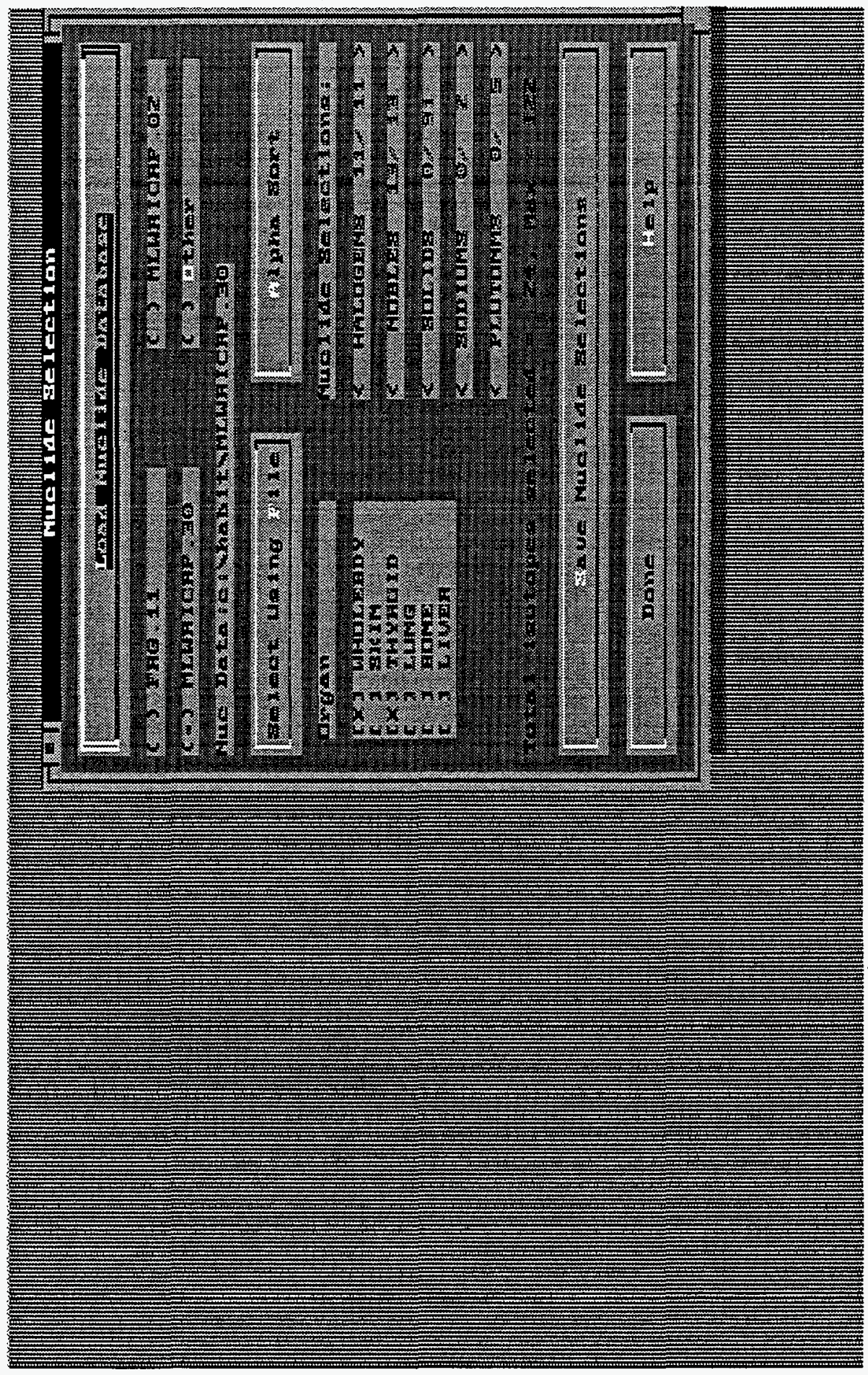

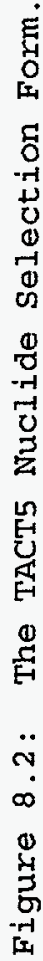




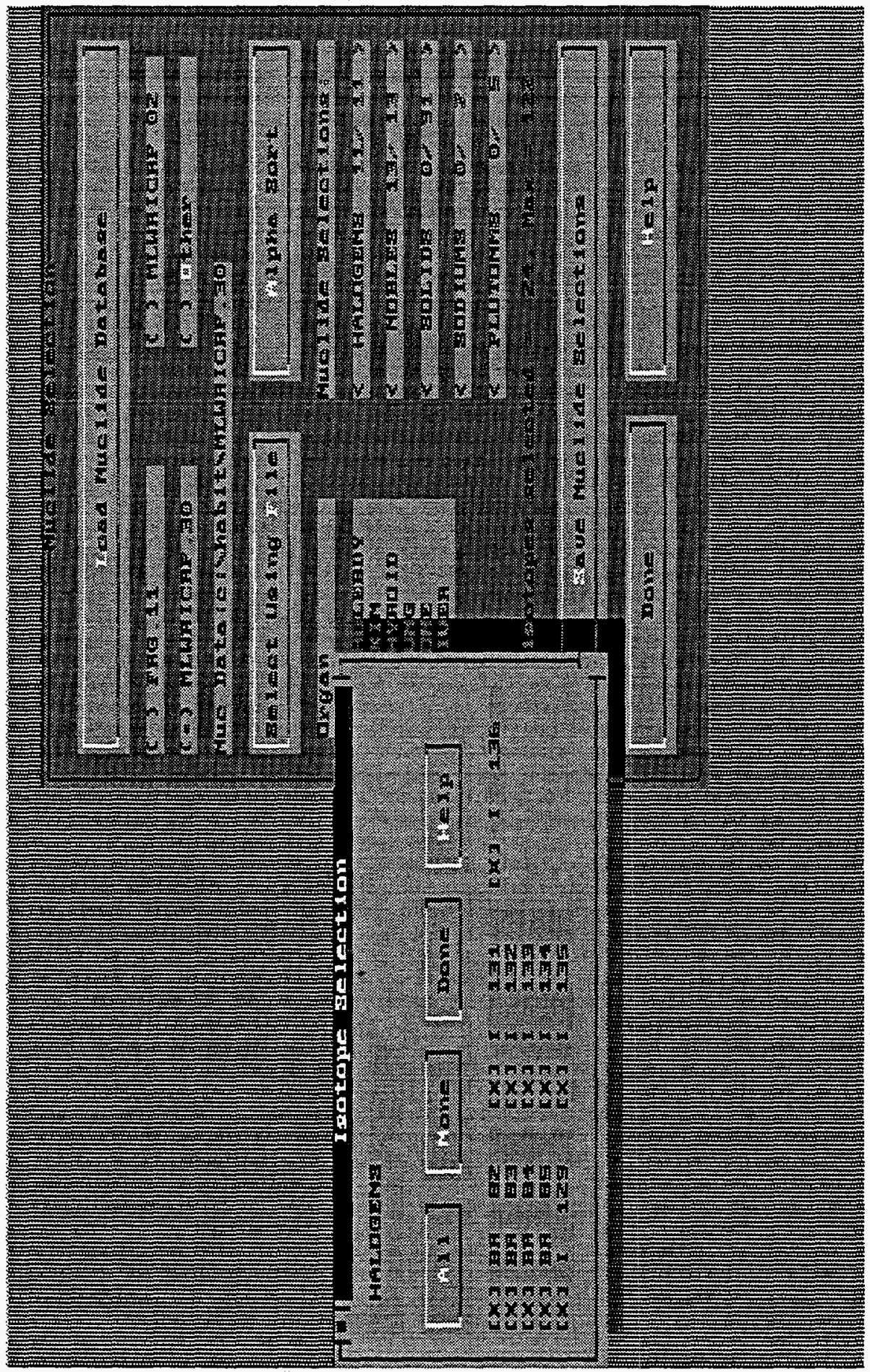

है 


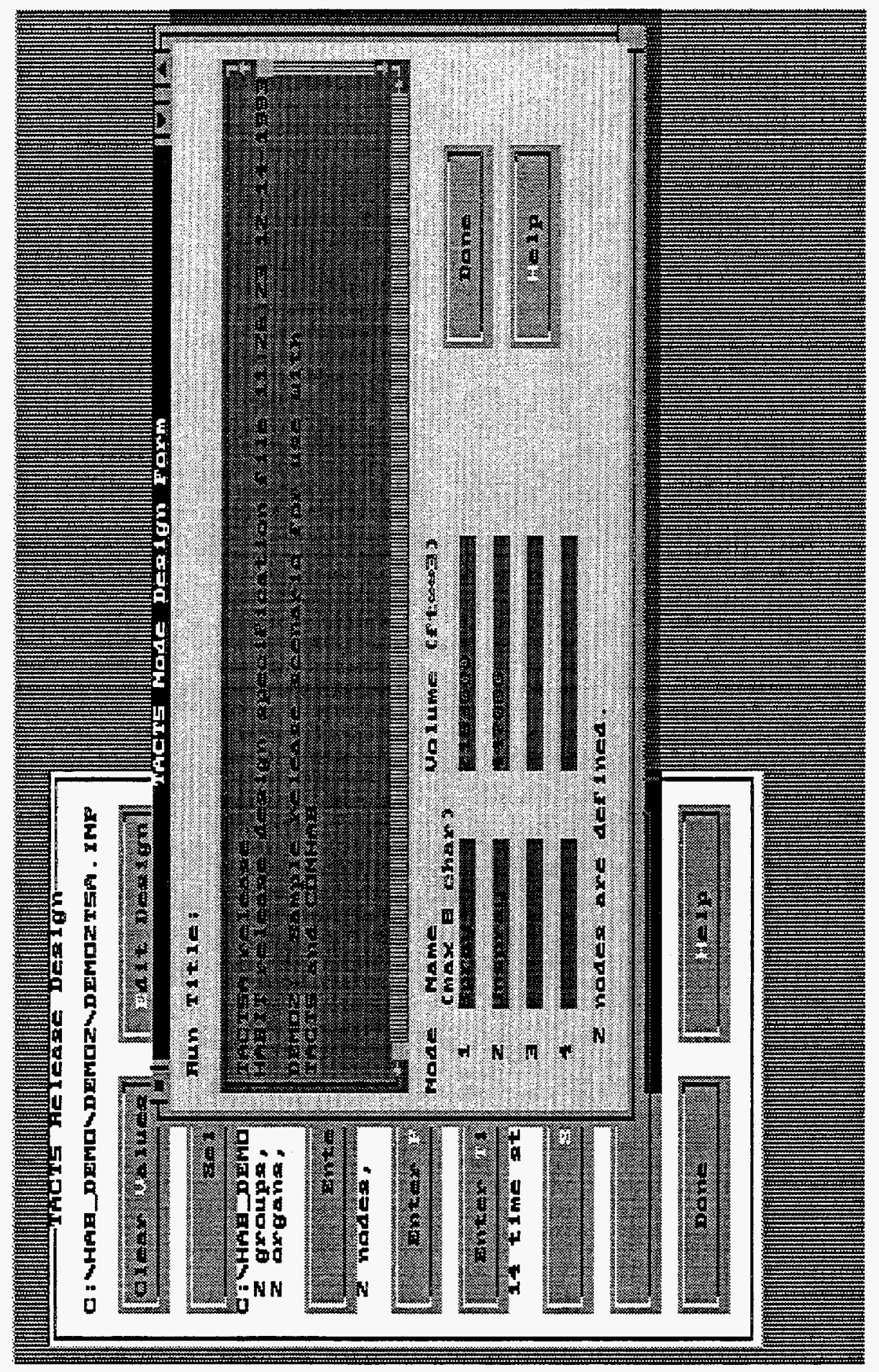

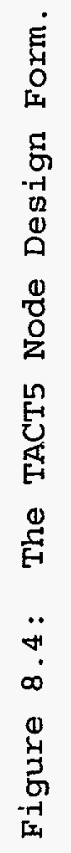




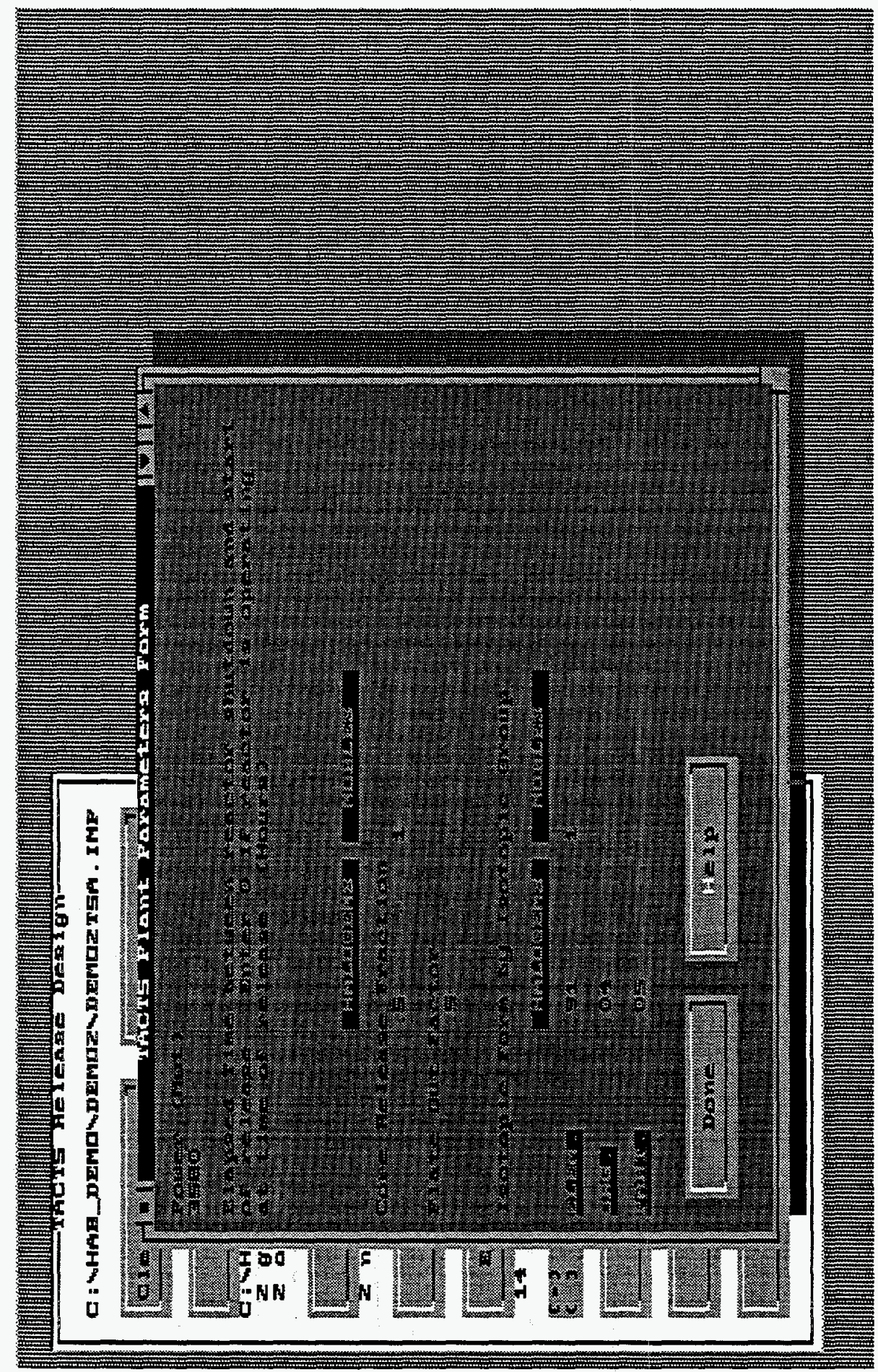

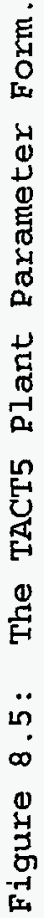




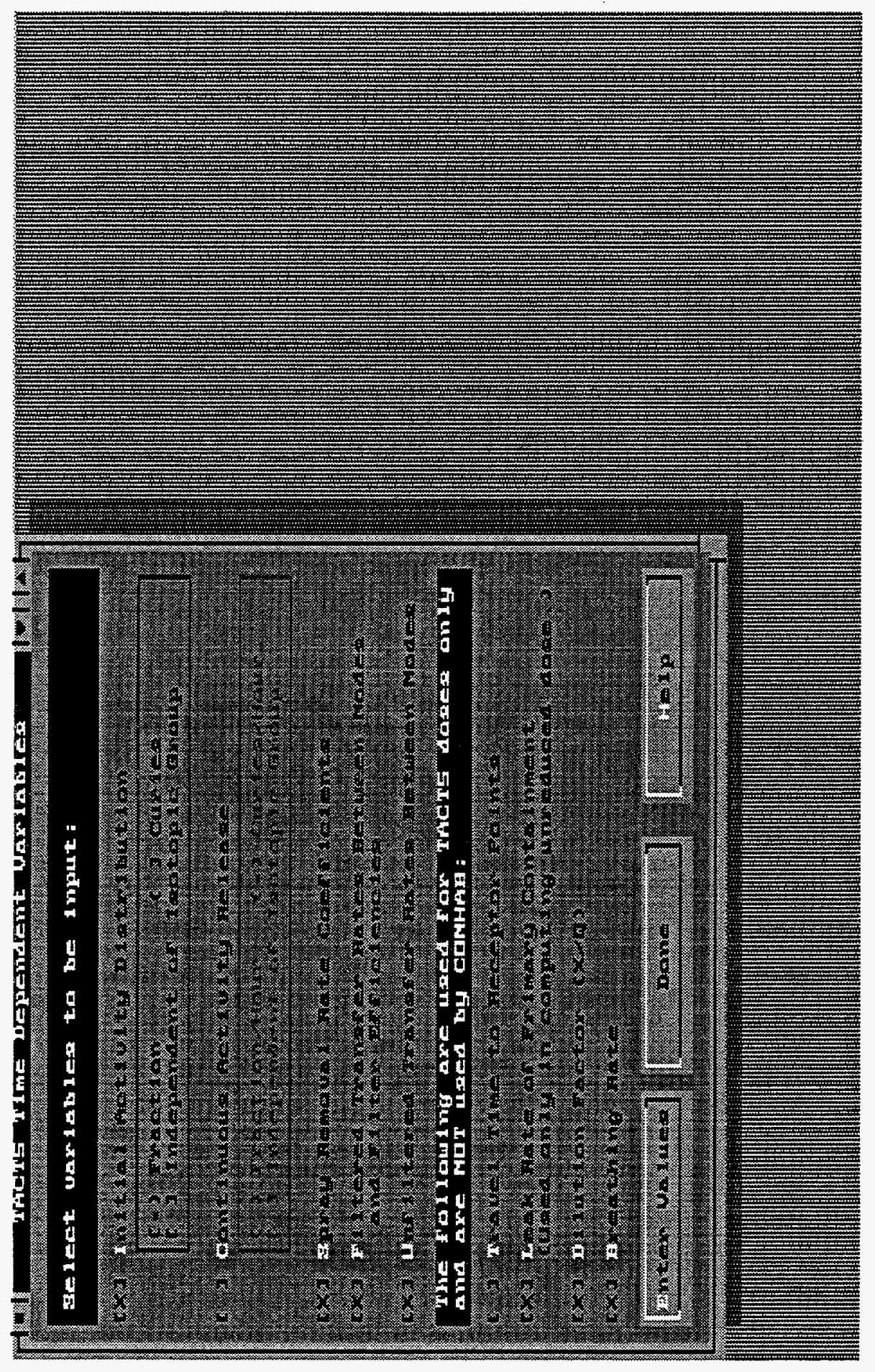

Ȯ 


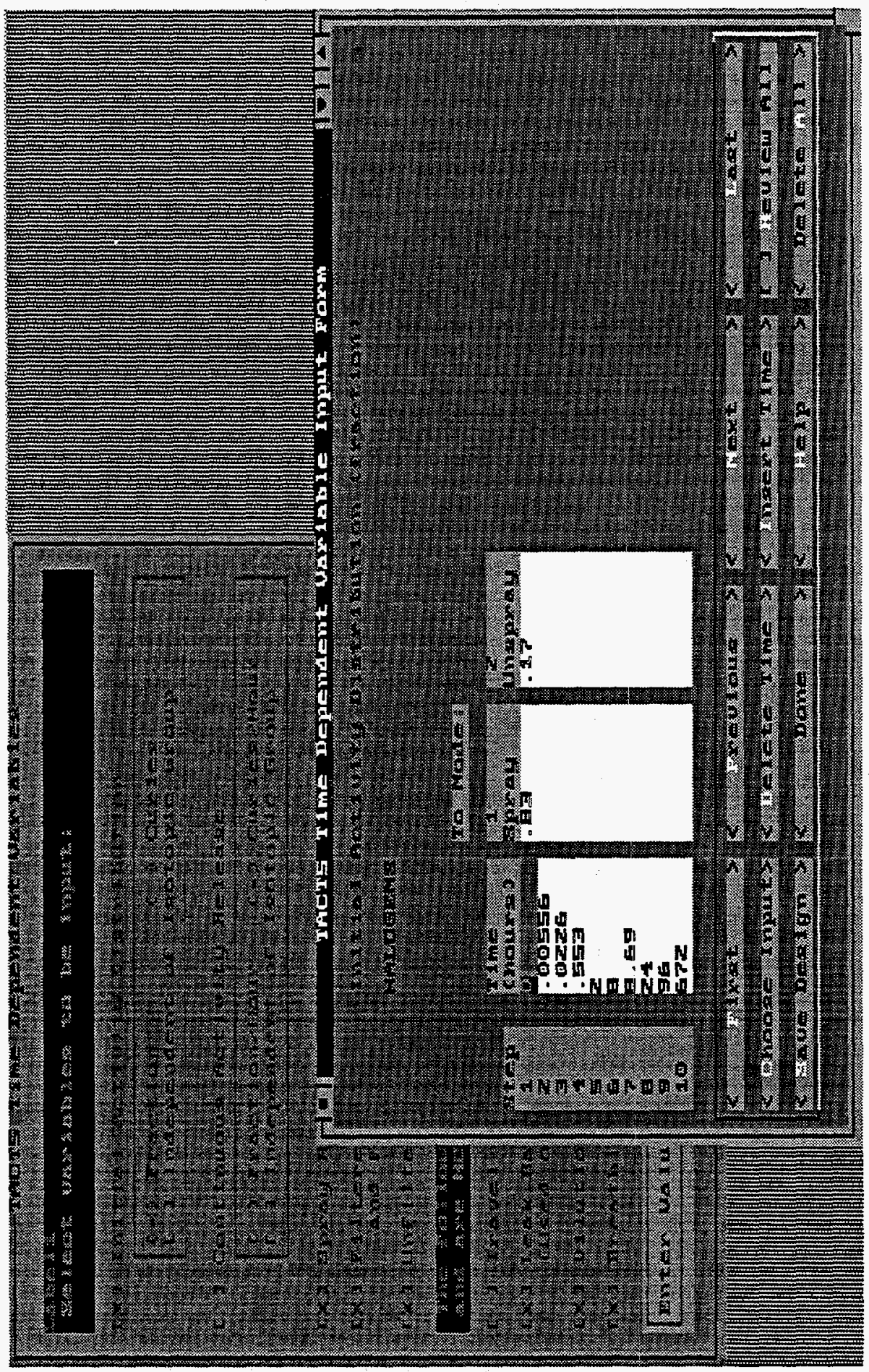

E

氝

点

0祭

बै -

댕

$>-1$

$\sqcup 0$

息势

펀

5

过公

品

0

至

담

近

E -

Q

兵

O

$\ddot{0}$

․ E

$\infty$ 突

ตั

然 


\author{
9. FPFP 2: \\ A CODE FOR FOLLOWING \\ AIRBORNE FISSION PRODUCTS IN \\ GENERIC NUCLEAR PIANT FLOW PATHS.
}

This program was described by Owcarski, et al. (1991). Several of the following sections on FPFP 2 have been taken from Owcarski, et al. (1991). In some cases they have been Eaken verbatim, in others they have been edited or rewritten to conform to the new version of FPFP_2. The data input sections of the FPFP_2 code and the associated text have been completely revised.

\title{
9.1. General Description of FPFP 2.
}

FPFP 2 is designed to compute the movement of radioactive fission products flowing through a plant along a single linear flow path. The concentration of fission products along the path is computed in units of fraction of the core per cubic foot.

\subsection{The Technical Basis for FPFP 2.}

Figure 9.1 is a schematic representation of the flow path used by FPFP_2. The figures for FPFP 2 show the way the screens appear when running DEMO3. The path consists of a sequence of spaces and nodes. The spaces, which represent elements of the plant, can be one of five types: a room, a duct, a filter, a resistance, or the end of the flow path. These differ in the assumptions made about the physical processes that are important within them. Between each pair of spaces is a node. The nodes serve to transfer air from one space to the next and allow for possible introduction of uncontaminated air into the flow at the node and for possible leakage of contaminated air from the node out of the flow path.

Fission products from the core are introduced into the first space of the flow path either as a puff injected at time $t=0$ (in which case the first space must be a room), or as continuous flow of activity into the first space.

A description of the physical processes assumed to take place in each type of space follows. Table 9.1 summarizes the physical processes used by FPFP_2 for each of these types of spaces.

\subsubsection{Rooms.}

FPFP 2 assumes that the gasses entering a room become well mixed with the air in the room and that gasses leave the room at the well-mixed concentration. Rooms not only dilute the incoming gasses, they can remove iodine by surface deposition, particles by gravitational settling, and particles by liquid sprays. Spray washout of iodine is not modeled here.

\subsubsection{Ducts.}

A duct is a flow path component where gasses enter at one end of a long constant cross-section system and proceed to an exit. There might be situations where it is difficult to decide whether a component is a room or a duct. Situations where a duct has many turns or cross section changes will require engineering judgment to arrive at an equivalent constant cross-section 
duct.

FPFP_2 assumes that plug flow exists in a duct. This assumption precludes any diffusion in the flow direction and simplifies the FPFP_2 program. Iodine and particles are allowed to deposit on all surfaces and upw̄ard-facing surfaces, respectively. Turbulent diffusion, diffusiophoresis, thermophoresis, and Brownian diffusion of particles are not modeled in FPFP_2.

\subsubsection{Filters.}

Filters remove a fraction of the radioactive material passing though them. The user must supply the appropriate filter efficiencies for halogens and solids. Noble gasses are not effectively removed by filters. FPFP 2 assumes that filters have negligibly small volume and therefore do not dilute the gasses flowing through them.

\subsubsection{Resistances.}

A flow resistance is a convenience for adjusting the flow rates between two nodes. Flow resistances have negligible volume. They have no removal or dilution. They simply transmit the flow from one node to the next with no change in concentration.

\subsubsection{End of Flow Path.}

This is not really a space type at all. It is simply used to denote the end of the flow path. Concentrations of air arriving at the end of the flow path are written to the file ID---FPA.CNX or ID---FPB.CNX for use in CONHAB. 
Table 9.1: Physical Processes Modeled in FPFP_2.

\begin{tabular}{|l|l|l|l|l|}
\hline \multicolumn{2}{||l|}{ Space Type: } & \multicolumn{3}{|l|}{} \\
\hline & $\begin{array}{l}\text { Surface } \\
\text { Deposition }\end{array}$ & Settling & Mixing & $\begin{array}{l}\text { Spray } \\
\text { Removal }\end{array}$ \\
\hline Halogen & Yes & No & Yes & No \\
Noble Gasses & No & No & Yes & No \\
Solids & No & Yes & Yes & Yes \\
\hline
\end{tabular}

\begin{tabular}{|l|l|l|}
\hline \multicolumn{2}{||l|}{ Space Type: } \\
\hline & $\begin{array}{l}\text { Surface } \\
\text { Deposition }\end{array}$ & SettIing \\
\hline & Yes & No \\
Halogen & No & No \\
Noble Gasses & Yes \\
Solids & No & You \\
\hline
\end{tabular}

\begin{tabular}{|l|l|}
\hline Space Type: & Filter \\
\hline & Filtration \\
\hline Halogen & Yes \\
Noble Gasses & No \\
Solids & Yes \\
\hline
\end{tabular}




\subsection{Fission Product Surface Deposition, Settling, and Mixing.}

All fission products that can be removed by natural deposition processes are either vapor molecules or fine particles. Organic ioäines are considered to be inert gasses (like noble gasses), except when they pass through certain absorbers. Iodine vapors (as $\mathrm{HI}$ or $I_{2}$ ) are treated as though they were all I. Therefore, any exposed surface will be considered as a deposition surface for $I_{2}$. Since most weli-aged accident aerosol particles are greater than 0.1 micron diameter (Gieske, et al., 1984; Denning, et al., 1986) and since accident flow rates leading to control rooms are anticipated to be close to normal duct flow values (i.e., slow), only gravity settling is considered as a removal mechanism apart from filtration.

If a user would like to consider high flow-rate turbulent deposition, that mechanism could be easily added to the code. Diffusiophoretic and

thermophoretic deposition mechanisms could be added, but these would require supporting heat transfer and condensation analyses as well.

\subsubsection{Surface Deposition and Mixing of Iodine Vapor.}

The "Halogens" group in FPFP_2 is used to model iodine vapor. Iodine vapor reaches deposition surfaces through diffusion through a boundary layer. The deposition velocity, $\mathrm{k}$ ( $\mathrm{ft} / \mathrm{min}$ ), is obtained from correlations for heat transfer coefficients using the Chilton-Colburn analogy for mass transfer coefficients (Bird, Stewart, and Lightfoot, 1960). The Sherwood number (Sh) becomes a function of the Schmidt number (Sc), the Reynolds number (Re), and the friction factor ( $f$ ):

$$
\mathrm{Sh}=\mathrm{Sc}^{1 / 3} \operatorname{Re} \mathrm{f} / 2 \text {. }
$$

For turbulent duct flow,

$$
f=0.0791 \mathrm{Re}^{-1 / 4} \text {. }
$$

Other definitions are

$$
\begin{aligned}
& \mathrm{Sh}=\mathrm{kd} / \mathrm{D}, \\
& \mathrm{SC}=\mu / \mathrm{D}, \\
& \mathrm{Re}=\mathrm{rdv} / \mu, \\
& \mathrm{d}=4 \mathrm{R}, \\
& \mathrm{R}_{\mathrm{h}}=\text { the hydraulic radius, } \\
& \mathrm{D}=\text { iodine diffussivity, } \\
& r=\text { air density, } \\
& \mathrm{v}=\text { air velocity, } \\
& \mu=\text { air viscosity. }
\end{aligned}
$$


Because the above relationships do not account for surface roughness and bends in ducts, $k$ values will be conservatively low. Values for $k$ will also be conservative when these equations are used to estimate $k$ in rooms. After introducing the deposition velocity for mass transfer, we now examine how it is used.

The differential equation for fission product concentration (c) in a wellmixed room is

$$
V(d c / d t)=F_{i} c_{i}-\left(F_{0}+k A\right) c
$$

where

$$
\begin{aligned}
& \mathrm{V}=\text { room volume, } \\
& \mathrm{F}_{\mathbf{i}}=\text { inlet flow rate, } \\
& \mathrm{C}_{\mathbf{i}}=\text { inlet concentration, } \\
& \mathrm{F}_{0}=\text { outlet flow rate, } \\
& \mathrm{A}=\text { room surface area. }
\end{aligned}
$$

Note that $F_{i}$ and $F_{0}$. like other flow rates in FPFP_2, are in actual cubic feet
per minute $(A C F M)$. The partial differential equation for concentration in a duct along the $x$ (flow) direction (neglecting diffusion) is

where

$$
\delta c / \delta t=-v \delta c / \delta x-k P c / A_{x}
$$

$$
\begin{aligned}
& v=\operatorname{air} \text { velocity, } \\
& A_{X}=\text { cross-sectional area, } \\
& P=\text { perimeter of } A_{x} .
\end{aligned}
$$

The above two differentiai equations are solved using a series of time steps over the duration of the transient accident. The noble gasses and organic iodines follow the same equations except that $k=0$.

The equations above assume that the iodine vapor finds a deposition surface that readily adsorbs the iodine without reentrainment. Therefore, iodine deposition calculated by FPFP_2 is a upper-bound value. This ideal situation is probably realistic for cleān metallic and painted surfaces. However, certain materials can become saturated and reentrain iodine in the presence of lower iodine concentrations than the maximum observed during the accident This reentrainment behavior is material-specific and cannot be easily generalized. The user of FPFP_2 should be aware that iodine can significantly reentrain in the days following deposition. [See Unrein, et al. (1985) and

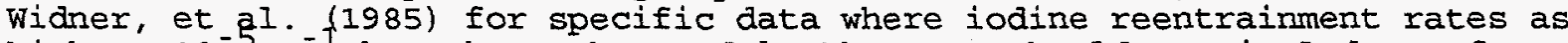
high as $10^{-5} \mathrm{~s}^{-1}$ have been observed.] The user should not include surfaces in the area $A$ for iodine deposition that cannot absorb iodine. 


\subsubsection{Settling and Spray Removal of Particles.}

The "Solids" group in FPFP_2 is used to model particles. Particles can deposit on surfaces by a number of mechanisms. These are Brownian diffusion, gravity settling, electrophoresis, thermophoresis, diffusiophoresis, turbulent deposition, inertial impaction, and interception. For typical well-aged nuclear-accident-generated aerosols, with little water vapor present in the gas (i.e., no condensation on walls or particles), with small gas-to-wall temperature gradients, and with velocities that are not excessive, gravitational settling becomes the dominant deposition mechanism. FPFP_2 uses gravitational settling as the only particle deposition mechanism, and bécause the aerosols are well aged, particle agglomeration is ignored.

The differential equations in the previous section hold for particle settling with the following changes: $k$ becomes the particle settling velocity, $A$ becomes the area of the floor, and $P$ becomes the duct width.

The equations for deposition velocities have been established in other documents (Owcarski, Schrenk, and winegardner 1985). These velocities are a strong function of particle diameter and a less strong function of particle density. FPFP_2 represents the nuclear aerosol at its source as having an aerodynamic māss median diameter (AMM) of 1 micron with a geometric standard deviation of 2 . The aerosol particles are distributed into bins of five discrete particle sizes. FPFP_2 allows the user to apply a multiplier to the AMMD value. The particle size distribution above is typical of well-aged aerosols found by calculation in severe reactor accident analysis (Gieseke, et al., 1984; Denning, et al., 1986).

Reentrainment of particles is not considered in the models. The phenomenon is not important at the flow rate expected. A user could add models for this, if necessary. A reference for reentrainment is Ayer, et al. (1988).

The treatment of sprays in FPFP_2 has been changed since the version described by Owcarski, et al. (1991).

The current version uses an equation for spray removal coefficients that was developed by Powers, et al. (1993, p. 172) to give a good representation of existing spray systems. The values used are those for $\mathrm{m}_{f}=0.9$ without correction to other values of $\mathrm{m}_{f}$. Powers, et al. suggest that this value is adequate for many uses.

The equation used is

$$
\begin{aligned}
L=\exp (6 . & 83707 \\
& +1.0074 \quad \ln (\mathrm{q}) \\
& -4.1731 \mathrm{e}-3 \mathrm{q}^{2} \mathrm{~h} \\
& -1.2478 \mathrm{q} \mathrm{h} \\
& -2.4045 \mathrm{e}-5 \mathrm{q} \mathrm{h}), \\
& +9.006 \mathrm{e}-8 \mathrm{q}
\end{aligned}
$$


where

$$
\begin{aligned}
& I=\text { the spray removal coefficient (1/hour) } \\
& \begin{aligned}
\mathrm{q} & =\text { the spray water flux }\left(\mathrm{cm}^{2} / \mathrm{s}\right) \\
& =0.06790 \mathrm{~s} / \mathrm{A}
\end{aligned} \\
& \mathrm{h}=\text { the spray height (feet) } \\
& \mathrm{S}=\text { the spray flow rate (gallons/min) } \\
& \left.\mathrm{A}=\text { the floor area of the containment (ft }{ }^{2}\right) .
\end{aligned}
$$

\subsection{Starting FPFP 2 .}

FPFP_2 is started from the HABIT Main window by clicking the FPFP_2 button.

FPFP 2 may also be started outside of HABIT (i.e., in stand-alone mode) by typing FP at the DOS prompt. If you start FPFP 2 in this way, you will be working with the file names that would be used if you invoked FPFP 2 by pressing the button on the HABIT main window for FPFP_2 flow into unfiltered path 1 .

\subsection{The FPFP 2 Release Design Form.}

The FPFP_2 Release Design form is shown in Figure 9.2. A label at the top of the form telis the design currently being used. The controls on this form are:

The Clear Values button is used to clear all values from the FPFP_2 input and "start over with a clean sheet of paper."

The Edit Design button may be used to create a new design, to change the design being used, to copy a design or to delete a design. See instructions above on the use of the Design Edit Window.

The Run Title text box is used for the title of this run.

The Particle Diameter Multiplier value box. This is the aerodynamic mass median diameter (AMMD) expressed in microns to be used for particles in FPFP 2. As explained in the section on the treatment of particles, a good value for well-aged aerosols is 1.0 .

The Design Spaces button causes the FPFP_2 Space Design Form to appear so that parameters for spaces in the flow path can be specified. Note, all spaces of the flow design should be entered before entering the flow parameters.

The set Flow Rates button causes the FPFP 2 Flow Design Form to appear so that flow parameters can be specified.

The Save Design button causes the data to be written to a file. It can be used at any time to save your work. If you change your data and then click either Run FPFP 2 or Done you will receive a warning that your data has changed. The message will ask if you want to save your data. If you click Yes, your data will be saved before the run is made or 
before the program returns to the HABIT main window. If you click No, the file will not be saved and all changes will be lost. If you click Cancel, the Run or Done operation will be aborted.

The Run FPFP_2 button. Clicking this button causes FPFP 2 computations to be done. Information will be written to the screen about the files being used by FPFP_2 and about the progress of the computation. The user can abort the computation by pressing <ctrl>+C. A message will be written to the screen indicating whether the computation was successfully completed. After the computation is completed the user will be asked to press <Enter> to return to data entry for FPFP_2. press $H$ to return the HABIT main window, or press $X$ to exit HABIT (return to DOS).

The Done button is used to return to the HABIT main window.

The Help button is used to obtain help. See Using Help for more details.

9.6. FPEP 2 Space Design Form.

This form is used to specify the parameters for the various spaces that comprise the flow path. The spaces should be designed before the flows. As seen in Figures 9.3, 9.4, 9.5, and 9.6, the appearance of space design form depends on the type of the space.

The Space Number value box shows the number of the space being designed. Entering a value in this box and pressing <Enter> or <Tab> changes the space being displayed.

The space + button changes to the next space in the design.

The space - button changes to the previous space in the design.

The End Space label shows the number of the space that is the current end of the design.

The Space Type Selection option buttons are used to select the type of space: Choices are Room, Duct, Filter, and Resistance. When the space type is changed, the items shown on the remainder of the Space Design Form change to show those parameters that are needed for each type space.

Note that a resistance simply transports the flow without change in concentrations. No parameters are needed for a resistance. The main use for a resistance in a design is to join two nodes without otherwise effecting the concentrations. I.e., the concentrations in the flow path are changed by inflow and outflow at the nodes on either side of the a resistance, but not by the resistance itself.

The End space is not really a space at all, but is used to denote the end of the flow path. Setting the type of a space to "End" truncates the flow path by discarding spaces beyond that point.

The Dimensions frame has value boxes for Height, width, Length of the rooms and ducts. These numbers should be the values of an equivalent right parallelepiped. For example, Owcarski, et al. (1991) suggests, that a round duct of length $I$, volume, $V$, and radius $r$, where $V=\pi r^{2}$, 
be represented by a dyct of length. $I$, volume $V$, and flow cross section, $1^{2}$, such that $1^{2}=\pi r^{2}$.

The Puff release at Time $=0$ frame appears only for space one and then only if it is a room. When the Yes option button is selected, it indicates that a puff of activity is released into space 1 at time 0 . When the Yes option is selected, values can be entered for puff concentrations of Halogens, Noble Gasses, and solids (core fraction $/ \mathrm{ft}^{3}$ ).

The Active Sprays frame appears for rooms. The Yes and No option buttons indicate whether the room has active sprays. When the Yes button is chosen, values can be entered for Time on (min), Time off (min), Spray Height (ft), and Flow Rate (GPM) of the sprays.

NOTE: It is important to be sure to enter the spray time on and spray time off as time steps on the flow design form.

The Duct Angle frame allows entry of the angle of a duct in degrees from horizontal.

The Filter Efficiencies frame appears only for filters and allows entry of filter efficiencies for Halogens and solids in percent.

The Natural Deposition frame appears for rooms and ducts. The On, Off, and Enter Lambdas options in this frame determine the way that surface deposition and particle settling are handled in a space. When On is selected, surface deposition and particle settling are calculated according to Table 9.1 using the equations given in section 9.3 . When Off is selected, the calculation will be done assuming that there is no surface deposition and no particle settling in this space. When Enter Irambdas is selected, the user can enter values for removal rates (lambdas) of Halogens and Solids (fraction per hour).

The Help bution. See Using Help for more details.

The Done button.

\subsection{FPFP 2 Flow Design Form.}

This form is a schematic representation of the flow path. Spaces must be designed before using this form. The exact layout of the form depends on whether it is currently showing the first space of the flow path, the middle of the flow path or the end. Examples of the form for these three cases are shown in Figures $9.7,9.8$, and 9.9 .

HINT: The simplest way to use this form is to step through the form entering values and pressing <Enter>. The cursor will move to the next value box along the flow path. When the right side of the form is reached, the form will be redrawn to show the next part of the flow path. When the end of the flow path is reached, the form will be redrawn for the first space of the flow path and the next time step. One may then continue entering values for that time step.

The items across the top of this form determine the node number and the time step being displayed on the form. The middle of the form graphically shows the flows associated with the currently displayed nodes and spaces. The bottom of the form contains buttons for further manipulation of the data and 
the help and done buttons.

All flow rates within FPFP_2 are in units of actual cubic feet per minute (ACFM). This is the flow rate using the actual pressure and temperature of the flow, not corrected to standard conditions.

The items at the top of the form are:

The Node number value box shows the number of the node currently being displayed and can be used to change to another node.

The Node + button moves the display to the next node.

The Node - button moves the display to the previous node.

The step value box shows the step number of the time currently being displayed on the form and may be used to change to another time step.

The step + button moves the display to the next time step.

The step - button moves the display to the previous time step.

The Time (min) drop down list box is used to enter the time of the current step. To add a new time to the flow data, simply type the time in the box for this list. The time entered will then be inserted into the list of times. You can then either enter flow data for that time or you can enter additional times.

You must enter values for all times at which any flow parameter changes. The last time you enter will be the ending time for FPFP_2 computations. You do not need to enter any flow values for the last time you enter. You may also enter times at which you want FPFP 2 to write output. If space 1 contains active sprays, be sure to ente $\bar{r}$ the spray time on and the spray time off as time steps.

You can also use the time drop down list to change the time for which flow data is shown.

The Del Time button deletes all flow data for the time currently shown.

The Iast time defined label shows the step number and time of the last time that has been defined so far.

A label near the upper right of the form reminas the user that All flow rates are ACFM.

The layout of items across the midale of the form, which shows the flow parameters, depends on the node number being shown. Arrows represent direction of air flow and boxes surrounded by these arrows are value boxes for flow rates. The flows on this form proceed from the left to the right and from the bottom to the top.

If any step other than the first is displayed, then blue-gray labels appear below each value box showing the value of the parameter at the previous time step.

WARNING: Values must be entered for all parameters in the flow path. Any value not entered will be assumed to be zero. FPFP_2 does NOT assume that 
parameters continue to have the same values from the previous time. It is the responsibility of the user to be sure that all values have been entered. The Find Blank Value Button (see below) may be used to review a design to be sure that all values have been entered. The Use Previous button (see below) can also be used as an aid in entering values.

The items that may appear on this part of the form are:

The Continuous Source Inflow Concentration frame, which appears only when rode 1 is being displayed. It contains value boxes for entering inflow concentrations of Noble gasses, Halogens, and Solids in core fraction $/ \mathrm{ft}^{3}$.

Immediately to the right of the Inflow Concentrations frame is a value box for the flow rate into space 1 .

The space $\mathbf{N}$ frame shows the type of frame and has value boxes for Downstream Temperature (C) and Downstream Pressure (PSIG).

The temperature and pressure should be the values for the air leaving the space. These are used to calculate gas transport coefficients.

The Balance Space Flows button appears at the top of the space frame. It can be used to set the flow out of the space equal to the flow into the space. The rules used for the balance space flows button are:

1. If only the flow into space $\mathrm{N}$ has been specified, then that value is transferred to the flow out of space $N$ value box.

2. If only the flow out of space $N$ has been specified, then that value is transferred to the flow into space $N$ value box.

3. If both the flow into space $N$ and the flow out of space $N$ have been specified, then the values are checked to see if they are equal. If they are not equal, a message is printed.

To the right of the space $\mathrm{N}$ frame and to the left of the node $\mathrm{N}+1$ frame is the value box for Flow from space $N$ to node $N+1$.

Below the node $N+1$ frame is a value box for flow from the environment into node $N+1$ (Flow from outside to Node $N+1$ value box).

Above the node $N+I$ frame is a value box for flow from node $N+I$ to the environment (Flow from Node $\mathrm{N}+1$ to outside value box).

The value box for Flow from Node $N+1$ to space $N+1$ is located to the right of the node $\mathrm{N}+1$ node frame and to the left of the frame for space $\mathrm{N}+\mathrm{I}$.

The Balance Node Flows button is located within the node frame. This button is used to make sure that the flows specified at the node are in balance. The following rules apply when this button is clicked: 
HABIT Manual

NUREG / CR -6210

FPFP_2

1. If only the flow from the previous space into the node is specified, this value is transferred to the box for flow out of the node into the following space.

2. If only the flow out of the node into the following space is specified, this value is transferred to the box for flow from the previous space into the node.

3. If any two of the flows surrounding the node are specified, then the other two flows are given the values needed to balance the flows into and out of the node subject to the condition that none of the flows will be given a negative value.

4. If all four of the values have been entered, then they are checked for balance. A message is printed if they are not in balance.

NOTE: The balance node button is provided as a convenience in entering and checking the flows at a node, but it is the responsibility of the user to be sure that any flow rates produced by the button are appropriate.

NOTE: The FPFP 2 computational code uses the value for flow from space $\mathrm{N}$ to node $\mathrm{N}+1$, the value for flow from the outside into node $\mathrm{N}+1$, and the value for flow from node $N+1$ to space $N+1$. The value for flow from node $\mathrm{N}+1$ to the outside is not used.

The buttons at the bottom of the form are:

The Find Blank Value button moves forward to the next blank (unentered) value in the design. This may be used to check to see that all values have been entered for all flow parameters.

The Show/Hide Previous value button turns on/off display of previous values.

The Use Previous button allows the user to insert values from the previous time step into the design. The user will be given the choice of using all values from the previous time step or of using values from the previous time step only for those values that have not been entered for this time step.

The Done button is used to indicate that the user is through entering flow data.

The Help button. See Using Help for more details.

\subsection{Forms for FPFP 2 Data Entry.}

FPFP 2 does not readily lend itself to the use of standardized forms for data entry. It is suggested that you use the following steps when gathering data for use by FPFP 2 .

First make a schematic diagram of the flow path. Identify the type of each space. Table 9.2 shows the data needed for each type space.

Make a copy of your diagram for each time step. Add numbers as needed for inflow concentrations (core fractions per fth), for the flow rates between 
spaces and nodes, and for the flow rates from the environment into the nodes and from the nodes out to the environment. Also, include the pressures and temperatures of each space which is a room or a duct.

Table 9.2 Data Needed for FPFP 2 Spaces.

Room

Dimensions (feet): Length, width, height

Puff Release at Time=0: Yes/No

Concentrations (core fractions/ $f t^{3}$ ) Halogens, Nobles, Solids

Active Sprays: Yes/No Spray time on (min) Spray time off (min) Spray height (feet)

Flow Rate (GPM)

(If yes is selected.)

Natural Deposition: On/Off/Enter Lambdas Lambdas for Halogens and Solids (1/hr) (If enter lambdas is selected.)

Duct

Dimensions (feet): height, length, width

Duct Angle (deg)

Natural Deposition: On/Off/Enter Lambdas Lambdas for Halogens and Solids (1/hr) (If enter lambdas is selected.)

Filter

Filter efficiencies (percent)

Halogens, Solids

\section{Resistance}

No parameters required. 
HABIT ManuaI

NUREG/CR- 6210

FPFP_2

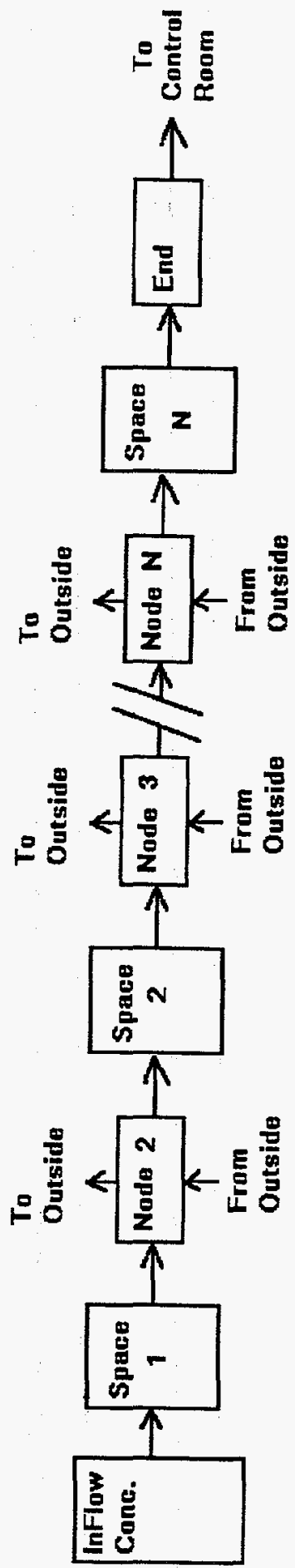

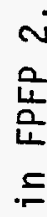

ปู

흥

$\stackrel{0}{\ddagger}$

4

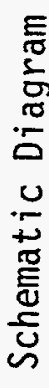

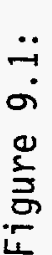




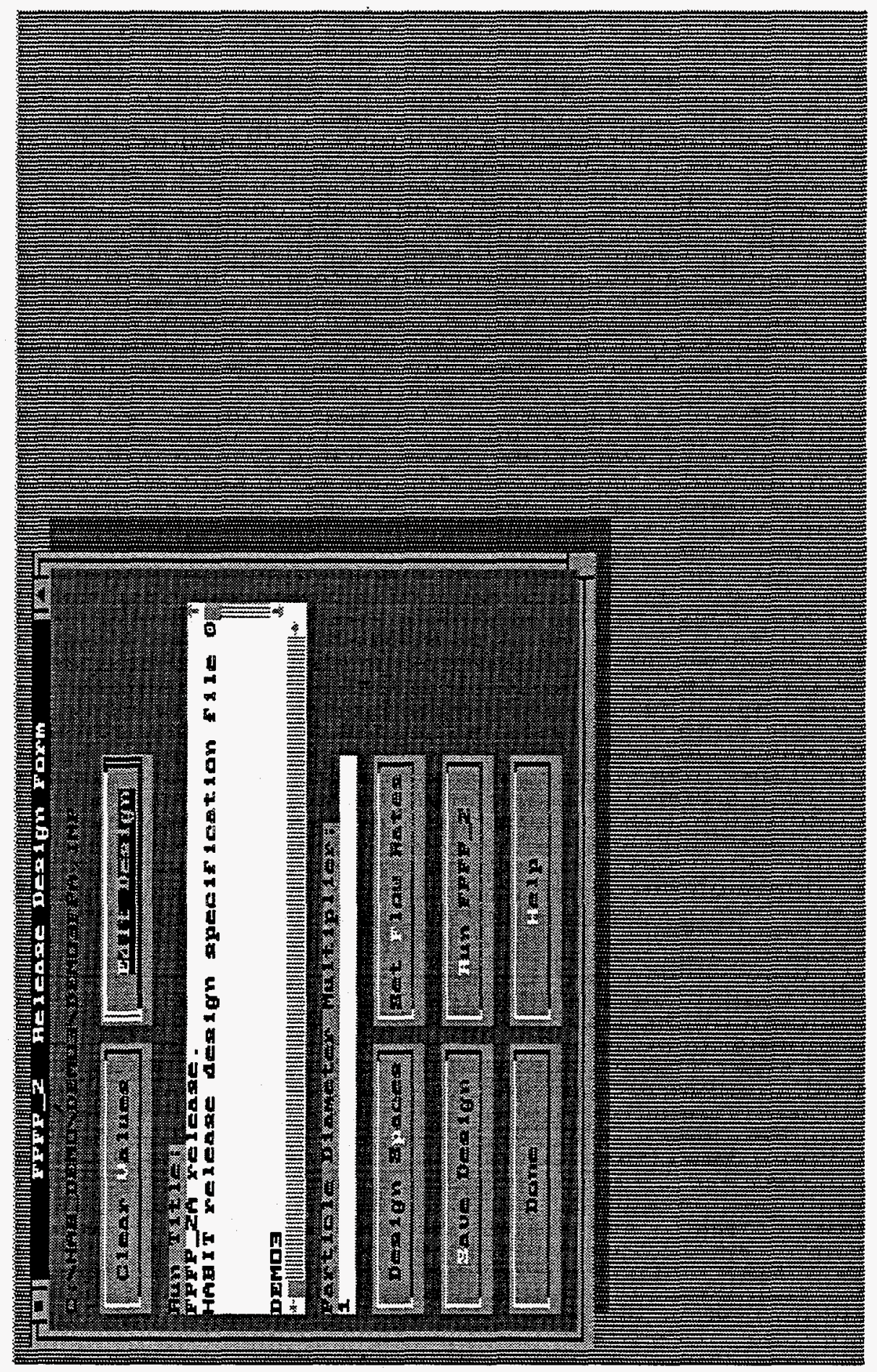

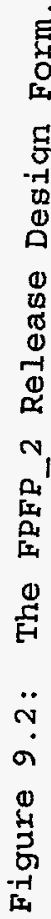




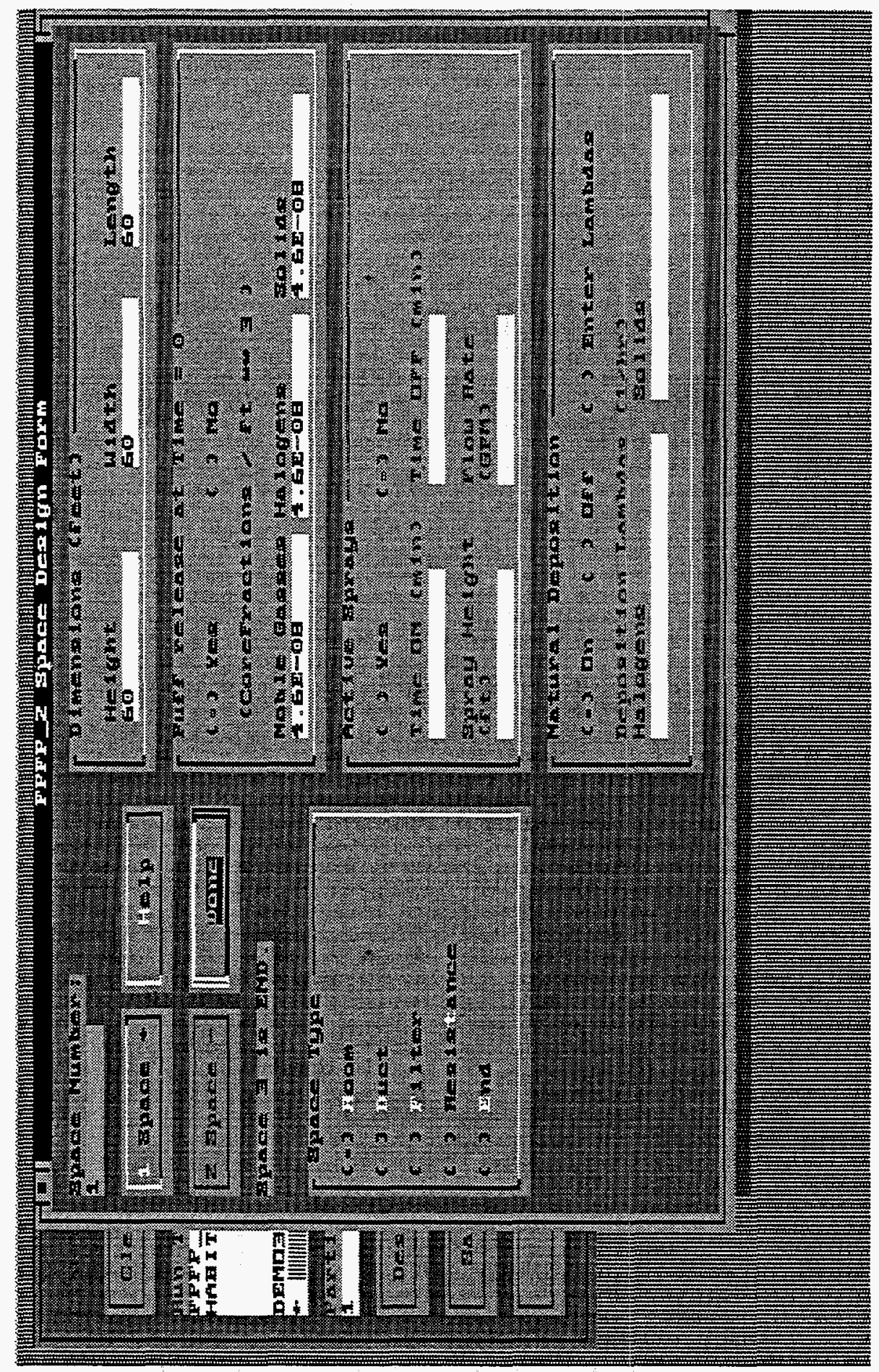

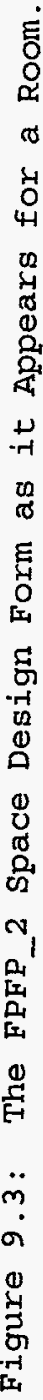




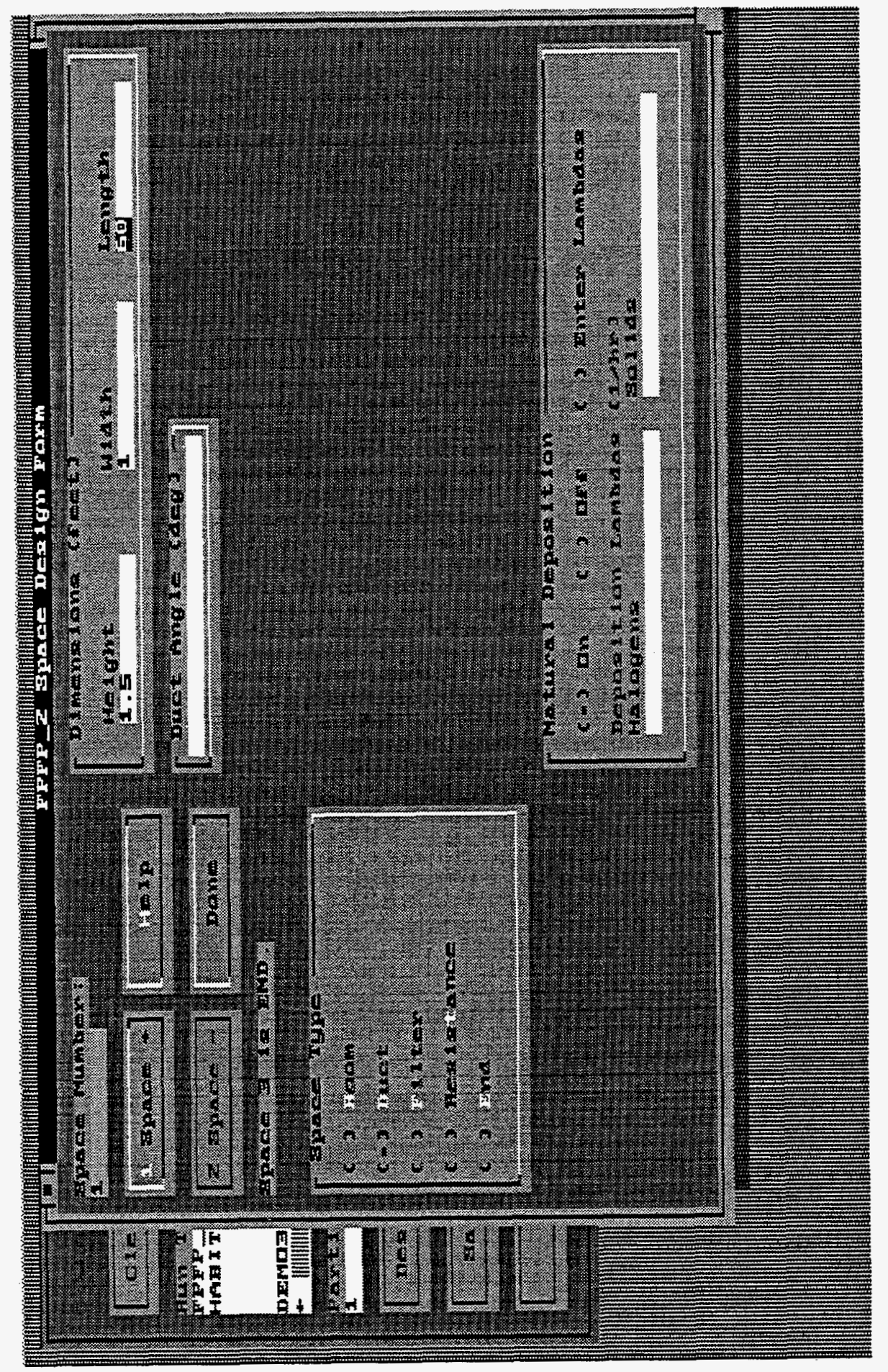

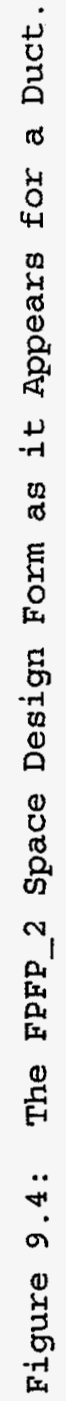




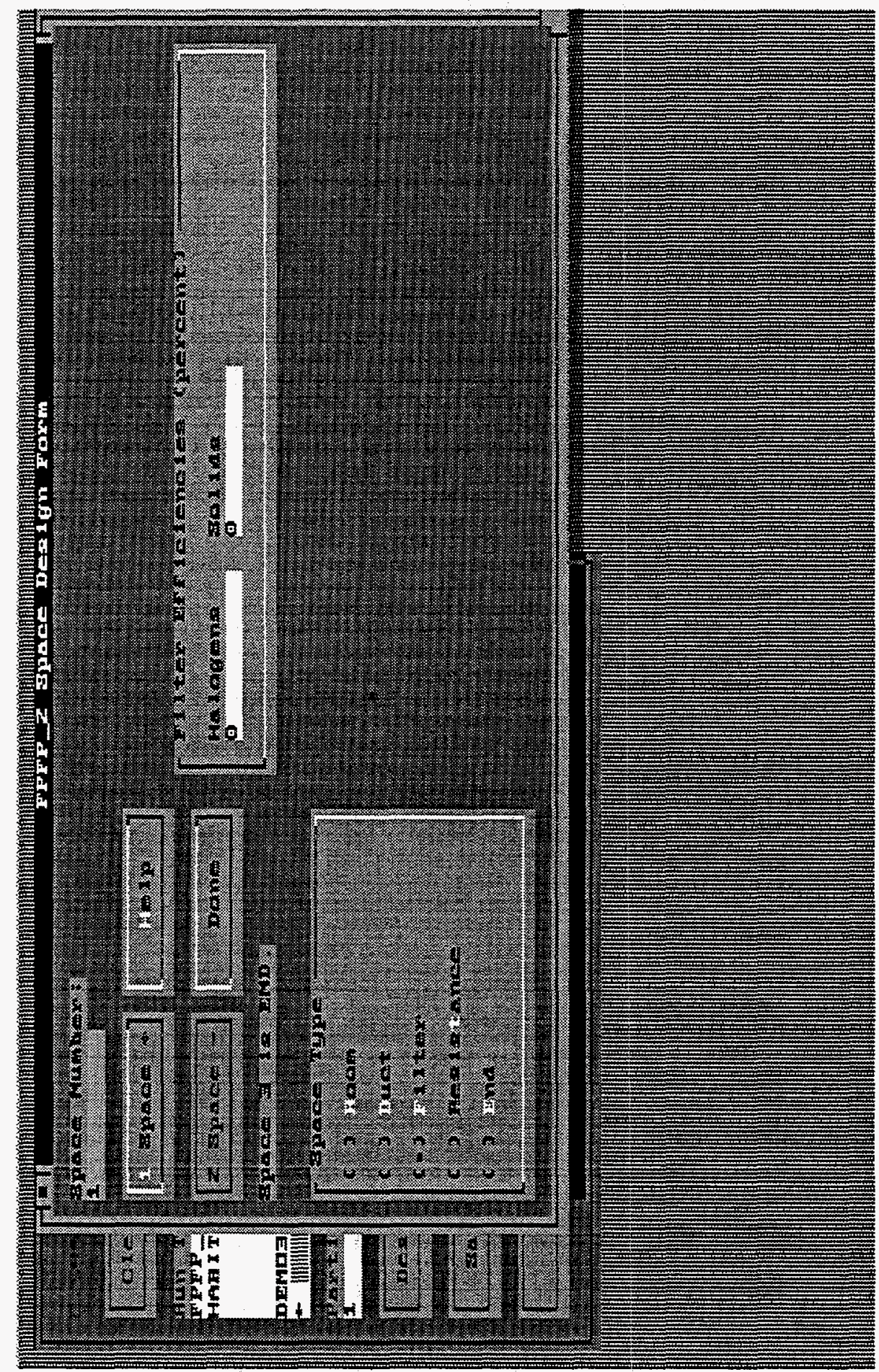

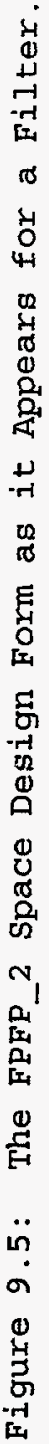




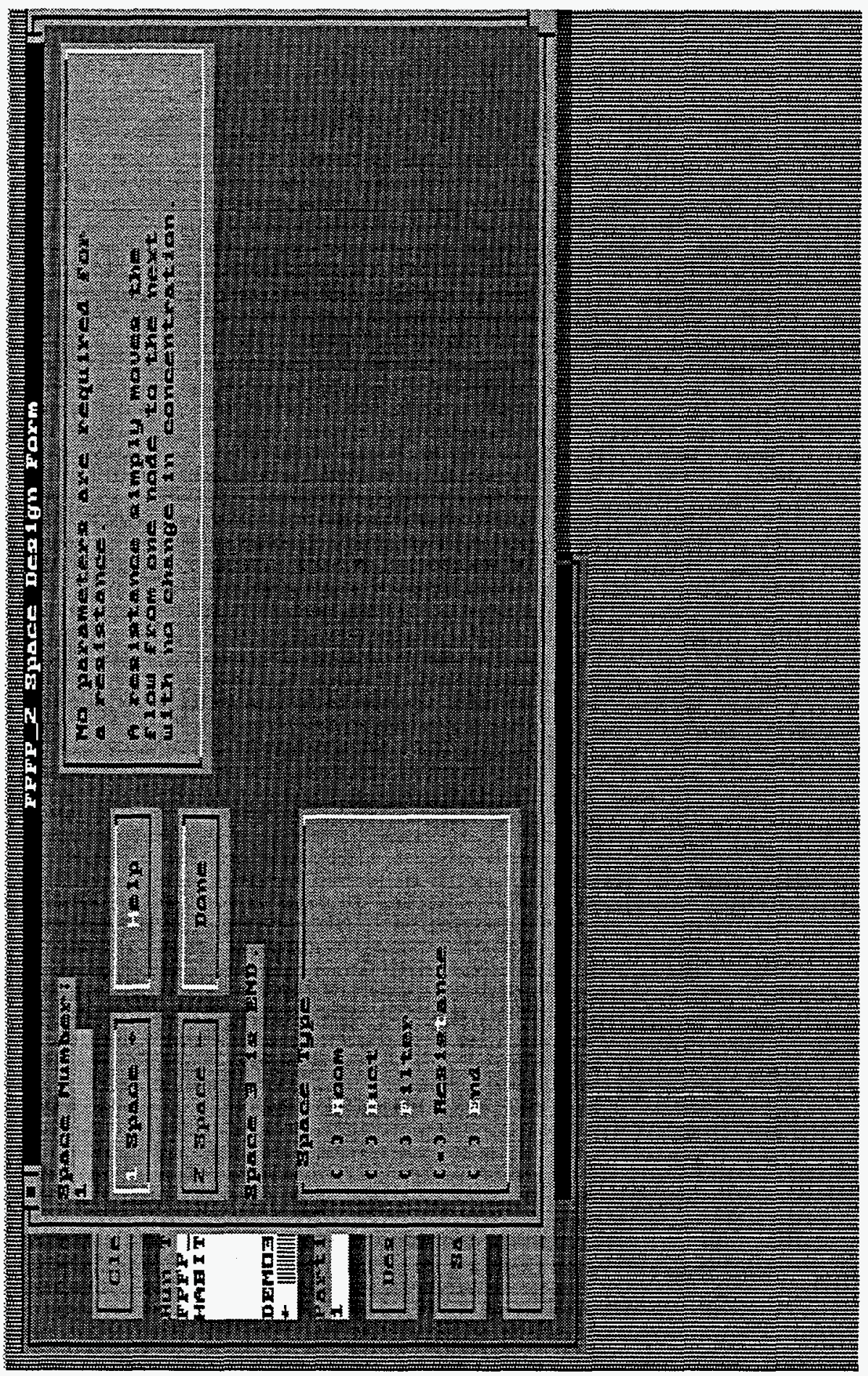

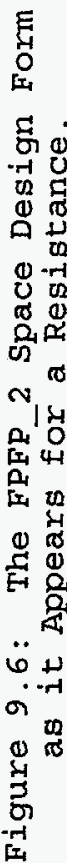




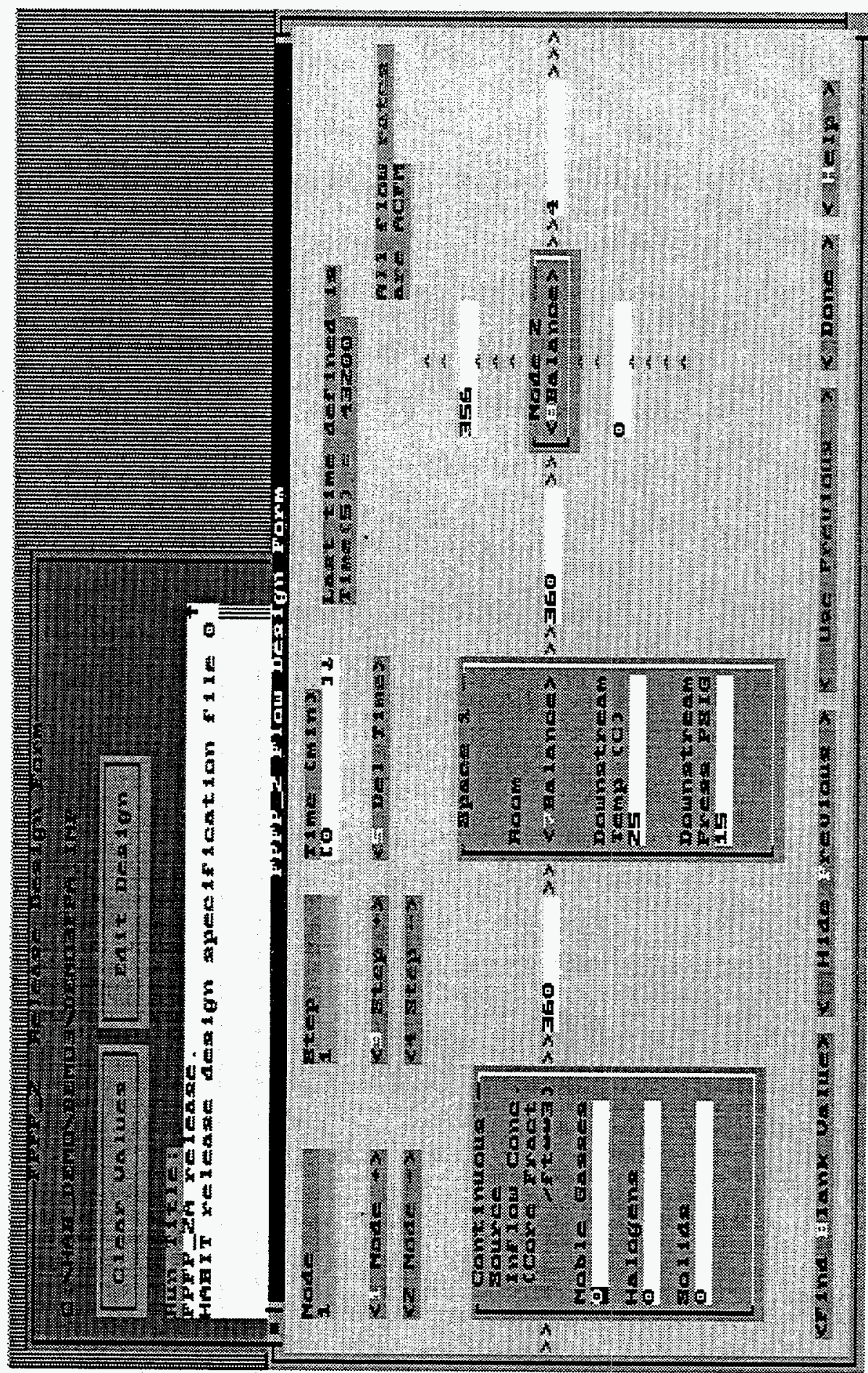

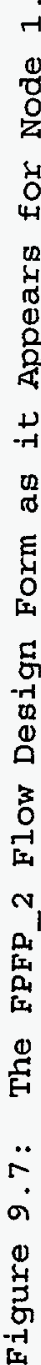




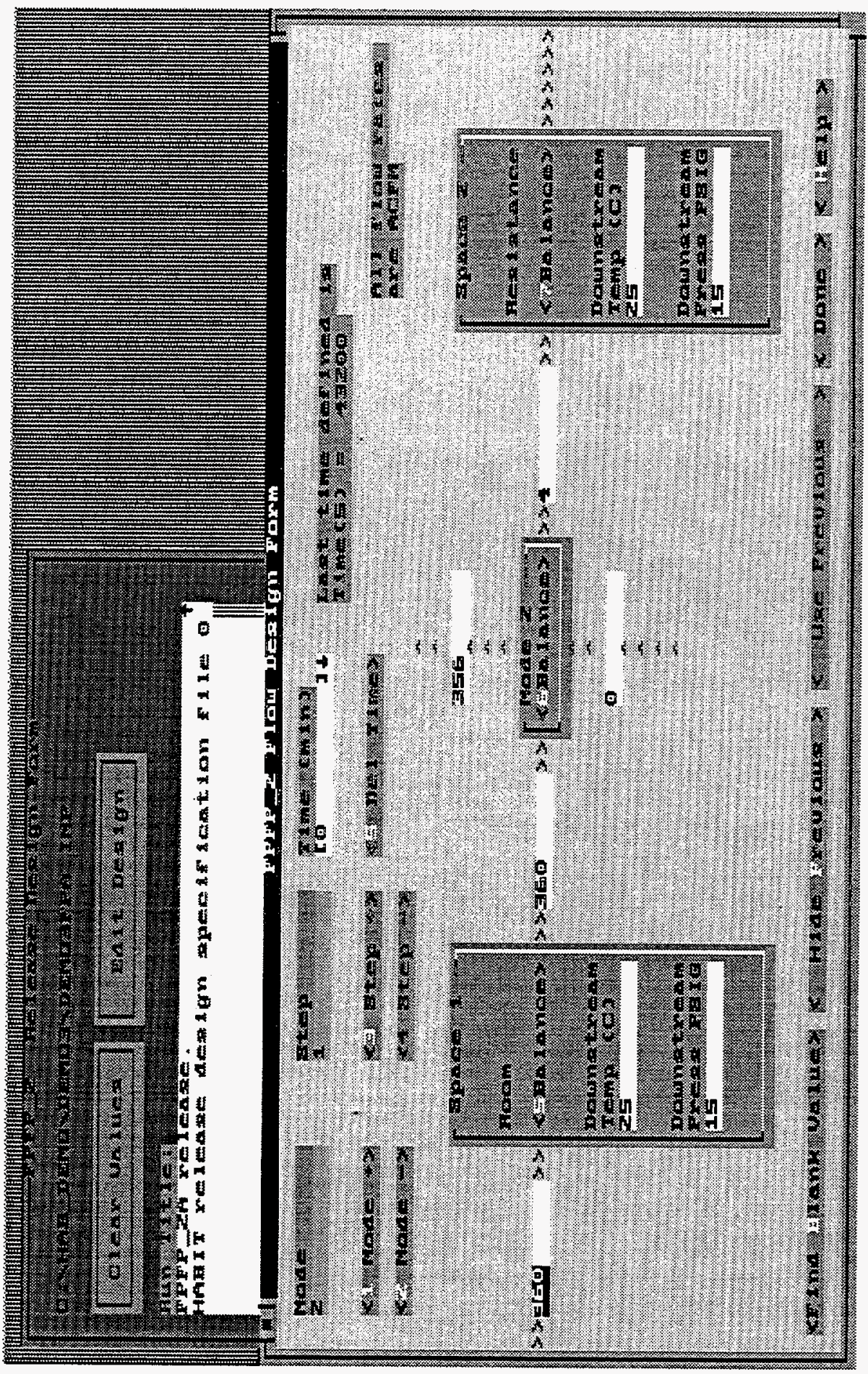

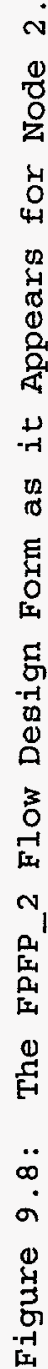




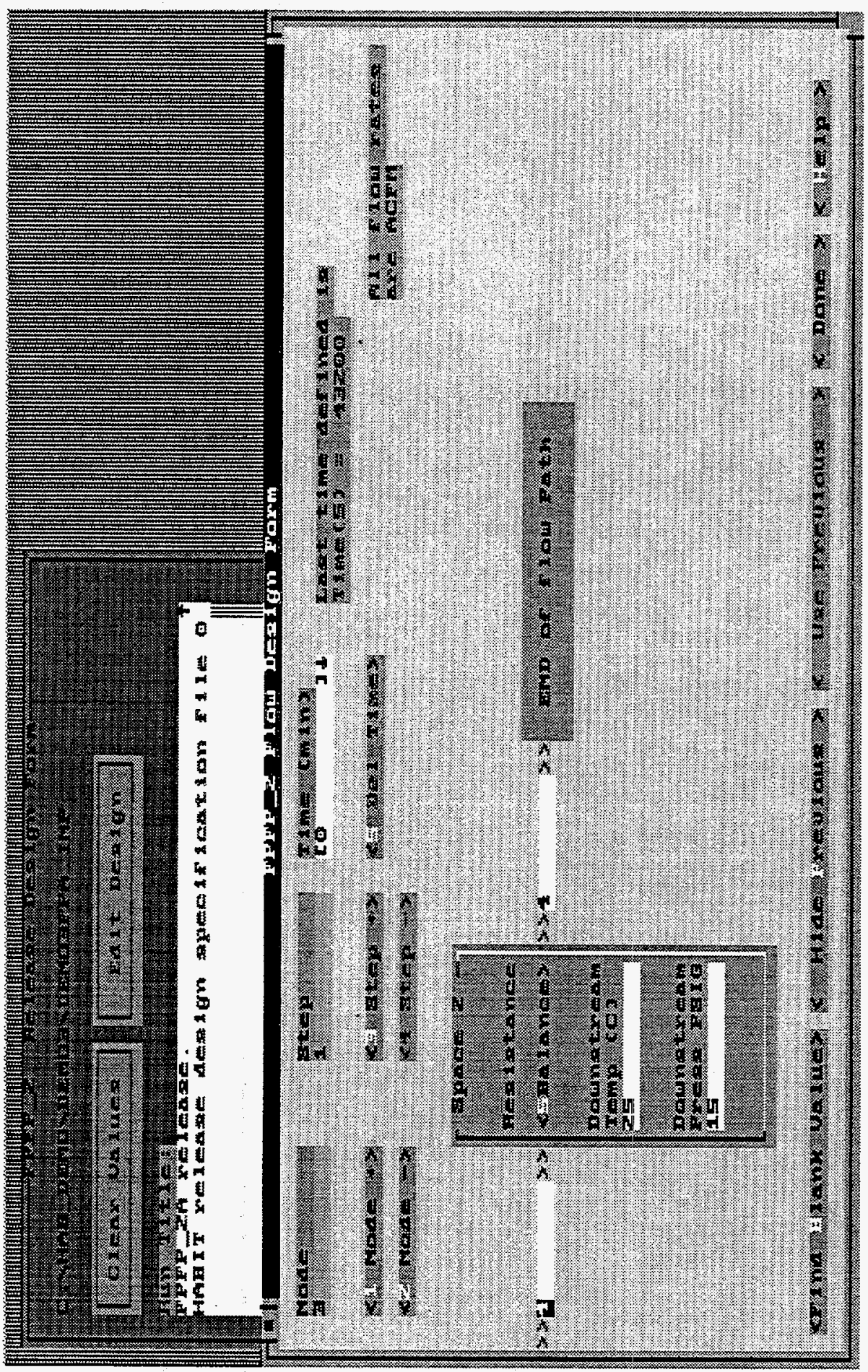

$m$
0
0
0
0

농

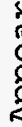

高

$\stackrel{-1}{-1}$

⿰氵

픙

เิ

E

ำ

吕

苔至

宦

N

哇

䄵

$\ddot{\sigma}$

i

岂 
The CONHAB program is described by Gilpin (1990). Several sections of the text that follows have been taken from that reference or are heavily based upon it.

\subsection{General Description of CONHAB.}

The transport of radioactivity to the control room boundary can occur either through the plant or through the atmosphere from the point of release to the point of entry into the control room boundary. An example of flow through the plant would be flow from the containment through rooms, ducts, and penetrations into the control room. Examples of flow through the atmosphere would be releases through containment leakage or stack flows with subsequent transport to the control room air intakes.

The FPFP_2 code is used to determine concentrations reaching the control room boundary by way of paths through the plant. The TACT5 code is used to calculate transport of radionuclides up to the point of release to the atmosphere. CONHAB performs a dispersion calculation to determine the resulting concentrations at the air intakes.

Most existing control room habitability systems can be modeled as a combination of one or more of the following systems:

I. Control room pressurization by filtered outside air can be used to reduce ingress of contaminated air.

2. The control room air can be recirculated through filters to remove contamination already in the control room volume.

3. Multiple widely-separated intakes can be monitored, and air from the least contaminated used to ventilate the control room.

4. Compressed air can be used to either pressurize the control room, or through breathing masks, supply uncontaminated air to the occupants.

5. The control room can be sealed to minimize the entrance of contaminants.

A system using any combination of these measures can be modeled using the schematic diagram for CONHAB shown in Figure 10.1. Filtered inflow path 1 and filtered inflow path 2 are used for intakes of atmospheric air and the concentrations arriving at the entrances to these two paths are obtained using values calculated by TACT5.

Unfiltered inflow path 1 and unfiltered inflow path 2 are used for flow paths through the plant. These inflow concentrations are computed using FPFP 2 . Although these are labeled as unfiltered inflow paths, filters at the inflow points can be modeled by simply including them as the last elements in the FPFP_2 flow paths. 


\subsection{Theoretical Basis for CONHAB.}

The assumptions in CONHAB are really quite simple. Activity is added to the control room through any of the four contaminated inflow paths. Activity is removed by the outflow of contaminated air, by the recirculating filter, and by radioactive decay. The air within the control room is assumed to be well mixed. The formation of daughter isotopes is included. Iodine vapor deposition on the surfaces of the control room and particle settling are NoT included in the model.

Let $A(t)$ represent the activity of a particular nuclide (in Curies) at time $t$ in the control room. The rate of change of activity for this nuclide can then can then be written as

$$
\begin{aligned}
& d A(t) / d t=\text { (rate of nuclide entering) - } \\
& \text { (rate of nuclide leaving). }
\end{aligned}
$$

Referring to Figure 10,1 , let $C_{i}$ represent the concentration $\left(\mathrm{Ci} / \mathrm{m}^{3}\right)$ at inflow $i, F_{i}$ the flow rate $\left(\mathrm{m}^{3} / \mathrm{s}\right)$ from inflow $i$ into the room, and $N_{i}$ the filter efficiency for filter $i$ for the nuclide in question. These symbols are shown in Figure 10.1. The rate of activity entering the control room from the plant and environment then becomes:

$$
R_{\text {in }}=C_{1} F_{1}+C_{2} F_{2}+C_{3} F_{3}\left(1-N_{3}\right)+C_{4} F_{4}\left(1-N_{4}\right)\left(1-N_{5}\right) \text {. }
$$

By conservation of mass the rate of air flow out of the control room is given by

$$
F_{\text {out }}=\mathrm{F}_{1}+\mathrm{F}_{2}+\mathrm{F}_{3}+\mathrm{F}_{4}+\mathrm{F}_{6} \text {. }
$$

The rate of activity leaving the control room is then

$$
R_{\text {out }}=F_{\text {out }}(\mathrm{A}(\mathrm{t}) / \mathrm{V})+\mathrm{F}_{5} \mathrm{~N}_{5} \mathrm{~A}(\mathrm{t}) / \mathrm{V} \text {, }
$$

where $V$ is the volume of the control room $\left(\mathrm{m}^{3}\right)$ and the second term is removal by the recirculating filter.

The differential equation for the time rate of change of activity then becomes:

$$
d \mathrm{~A}(\mathrm{t}) / \mathrm{dt}=R_{\text {in }}-\mathrm{A}(\mathrm{t})\left(\mathrm{F}_{\text {out }}+\mathrm{F}_{5} \mathrm{~N}_{5}\right) / \mathrm{V} \text {. }
$$

This equation can be readily solved over time intervals for which the inflow concentrations, flow rates, and filter efficiencies are constants.

$$
A(t)=A\left(t_{0}\right) K-A_{i n f}(I-K),
$$

where

$$
\begin{aligned}
& K=\exp \left(-\left(t-t_{0}\right)\left(F_{\text {out }}+F_{5} N_{5}\right) / V\right), \\
& A_{\text {inf }}=R_{\text {in }} V /\left(F_{\text {out }}+F_{5} N_{5}\right),
\end{aligned}
$$

and where $A\left(t_{0}\right)$ is the activity at the start of the time step. 
The activity is also reduced to account for the effects of radioactive decay and activity is added to account for formation of radioactive daughters during the time step.

The concentration in the control room at any given time is found from $C(t)=A(t) / V$.

The CONHAB code uses the above equations for each computational time step. The length of each time step is determined by the restriction that the inflow concentrations, flow rates, and filter efficiencies do not change during the time step. A further limitation is imposed to insure that the time steps are short enough to produce good computational accuracy. The details of how time steps are chosen are explained in chapter 13.

The concentrations at the intakes are not in fact constants, but are continuously varying functions of time. Nonetheless, the above equations can be used with good results because the versions of TACT5 and FPFP 2 used in HABIT write their results for time intervals that are short enough so that the inflow concentrations do not change very much during the time step.

The dose rate to a person in the control room is obtained by multiplying the concentration in the control room by a dose conversion factor, and the breathing rate for doses other than whole body or skin.

The dose rate is further reduced to account for the occupancy factor (i.e., what fraction of the 30 day period a person remains in the control room). The whole body dose is also reduced by a geometry factor taken from Murphy-Campe (1974) as $1173 \mathrm{~V}^{-} .338$ where the control room volume is in $\mathrm{ft}^{3}$. (CONHAB converts to $\mathrm{ft}^{3}$ internally.) This factor is an attempt to correct for the fact that the control room volume is a finite rather than the semi-infinite cloud of gamma emitters upon which the dose conversion factors are based.

The contribution of this time step to the cumulative dose is obtained by integrating the dose rate over the time step using the dose rate at the beginning of the time step and at the end of the time step in the trapezoid rule.

Note that the following improvements have been made in the computational algorithm compared to the version of CONHAB used by Gilpin (1990):

Radioactive decay and daughter formation are now included within the time step rather than after it. This means that the calculated concentrations now more closely correspond to the actual concentrations at the ends of the time steps and that the effects of decay during the time step are included in the dose computations. The Gilpin (1990) version also had the possibility of producing inaccurate results if too long time steps were taken. The current version automatically chooses a time step suitable for good computational accuracy.

\subsubsection{Treatment of Isotopes and Forms within CONHAB.}

CONHAB computes control room concentrations for three chemical forms of each of the isotopes in the nuclide data file (FGR.11, ICRP.30, or ICRP.02, depending on the user's choice. See section 1.10). The three chemical forms that are considered are elemental, organic, and particulate.

Each of the isotopes is considered to be a member of one of the five isotopic groups: halogens, noble gasses, solids, sodiums, and plutoniums. 
It is important to understand this division by isotopic group and chemical form because it effects how CONHAB treats concentrations flowing into the control room based on TACT5 and FPFP_2 computations. The effectiveness of the control room filters is also specified based on this division. Understanding how CONHAB assumes that each of these groups and forms behaves can also provide insight into what measures may be effective in reducing the doses computed by CONHAB. For instance if examination of the of the REM per hour tables in the file ID---CB.CNX indicate that particulates are contributing a significant fraction of the total dose rate, then more effective filters may be helpful. However, filters will not have any effect towards reducing the part of the dose rate that comes from noble gasses.

\subsubsection{Treatment of Unfiltered Inflows from FPFP_2.}

FPFP_2 produces inflow concentrations of core fraction per cubic foot for noble gasses, halogens, and solids. CONHAB converts this into Ci/ft 3 of each isotope using data of $\mathrm{Ci} / \mathrm{MWt}$ found in the nuclide data base file (see section 1.10). The activity is divided into the 3 physical forms according to the group fractions entered on the CONHAB Plant Parameters form.

\subsubsection{Treatment of Filtered Inflows from TACT5.}

TACT5 produces the files ID---T5A.CNX, and ID---T5B.CNX containing the values of the rate of activity release ( $\mathrm{Ci} / \mathrm{sec}$ ) to the atmosphere of each form (elemental, organic, and particulate) of each isotope.

CONHAB can compute dilution factors, $\mathrm{X} / \mathrm{Q}$, based on values of meteorological parameters. The equations for $\mathrm{X} / \mathrm{Q}$. which are the same as those used in EXTRAN, include the effects of nearby building wakes. See the section on EXTRAN. These values of $X / Q$ are for the dilution of activity released by the situation modeled in TACT5 when it arrives at the intake to filtered path 1 or 2. The TACT5 activity release rates are multiplied by the dilution factors $(\mathrm{X} / \mathrm{Q})$ computed by CONHAB to obtain concentrations at the inflows to filtered paths 1 and 2 .

\subsubsection{How Filters are Assumed to Work within CONHAB.}

The CONHAB input includes filter efficiencies for each of the three chemical forms: elemental, organic, and particulate. The "Elemental" filter efficiency is used for the elemental form of isotopes in the halogens group. The "Organic" filter efficiency is used for the organic form of isotopes in the halogens group. The "Particulate" filter efficiency is used for the particulate form of isotopes in the halogens group and for all three chemical forms of isotopes in the sodiums, solids, and plutoniums groups. Noble gasses are not effectively removed by filters. 


\subsubsection{Atmospheric Diffusion used by CONHAB.}

The user may enter values for meteorological parameters and have CONHAB compute values for dilution factors $(\mathrm{X} / \mathrm{Q})$. Alternately, the user may entex $X / Q$ values directly. If meteorological values are entered, $X / Q$ are wake diffusion coefficients computed using equations derived by Ramsdell following an analysis of data from building-wake diffusion experiments (Ramsdell 1995 ). RamsdelI (1988, 1990a,b, 1995) present derivations of the equations for the wake diffusion coefficients.

\subsection{CONHAB Compared with the Murphy-Campe Method.}

CONHAB is a more detailed and more realistic simulation of the evolution of concentrations within the control room. The Murphy-Campe method is based on the long-time control room concentrations. ConHAB takes account of the fact that the control room air starts out clean and requires some time to approach the long-time concentration. This effect generaliy tends to reduce the control room concentrations computed by CONHAB compared with those found using the Murphy-Campe method.

The second factor affecting comparison is daughter formation. The estimates produced by CONHAB are increased due to doses associated with daughter nuclides. The Murphy-Campe method does not take account of daughter formation.

For the DEMO2 sample run provided with HABIT (see section 11.2), these two factors nearly offset each other. In general, the dose estimates produced by CONHAB may be higher or lower than those produced by the Murphy-Campe method depending on the relative importance of these two effects.

\subsection{Starting CONHAB.}

CONHAB is started from the HABIT Main window by clicking the CONHAB button. It may also be started outside of HABIT ( $i . e .$, in stand-alone mode) by typing $C B$ at the DOS prompt. Before CONHAB is run, TACT5 and FPFP_2 must be run for all flow paths through which radionuclides enter the control room. If the "Use same data for filtered paths $1 \& 2$ " check box is checked and if the file ID---T5A. CNX exists then ID---T5A.CNX is copied to ID---T5B. CNX when the CONHAB button is clicked.

CONHAB attempts to find the files:

$$
\begin{aligned}
& \text { ID-- T5A.CNX, produced by TACT5 for filtered inflow } 1 \\
& \text { ID---TSB.CNX, produced by TACT5 for filtered inflow } 2 \text { (recirc) } \\
& \text { ID---FPA.CNX, produced by FPFP_2 for unfiltered inflow } 1 \\
& \text { ID---FPB.CNX, produced by FPFP_2 for unfiltered inflow } 2
\end{aligned}
$$

NOTE: If any of these files are not found, and the user has specified a nonzero flow rate for these paths, then CONHAB assumes that the air flowing into that path contains no radioactivity.

NOTE: It is important to be sure to run TACT5 and FPFP_2 for Iong enough. If TACT5 or FPFP_2 is run for less than 30 days (720 hours), CONHAB will use the last concentration from the TACT5 or FPFP_2 run as the intake concentration until the end of 30 days. You should therefore be sure that one of the following conditions is met: 1. TACT5 and FPFP 2 are run for at least 30 days, 2. TACT5 and FPFP_2 are run until concentrations are negligible, or 3. TACT5 and FPFP_2 are run until the flow into the control room becomes zero for 
HABIT Manual

NUREG/CR - 6210

CONHAB

the corresponding inflow path.

10.5. The CONHAB Main Form.

The CONHAB Main Form is shown in Figure 10.2. The remaining figures for CONHAB show the way the screens appear when running DEMO2. A label at the top of the form telis the design currently being used. Note that CHEM and CONHAB share the input file ID---CB.INP. Run title, control room volume, air flow rates, and occupancy factor appear on data entry forms for both CHEM and CONHAB and are used by both of these programs. Filter efficiencies, meteorological parameter values, reactor power, and group fractions appear only on the CONHAB data entry forms and are used only by that program.

The controls on this form are:

The Clear values button is used to clear al: values from the CONHAB input and "start over with a clean sheet of paper."

The Edit Design button may be used to create a new design, to change the design being used, to copy a design or to delete a design. See instructions above on the use of the Design Edit Window.

The Enter Plant Data button causes the ConHAB Plant Parameters form to appear.

The Enter Meteorology or $\mathrm{X} / \mathrm{Q}$ Data button causes the CONHAB Meteorological or $\mathrm{X} / \mathrm{Q}$ Parameters form to appear.

NOTE: Meteorology data is used in conjunction with flows through filtered path 1 and filtered path 2 ( $i . e .$, from TACT5). If there is no flow of contaminants through either of these paths, then you do not need to enter any meteorological data.

The Enter Control Room Flow Data button causes the CONHAB Control Room Flow Parameters form to appear.

The Save Design button causes the data to be written to a file. It can be used at any time to save your work. If you change your data and then click either Run CONHAB or Done you will receive a warning that your data has changed. The message will ask if you want to save your data. If you click Yes, your data will be saved before the run is made or before the program returns to the HABIT main window. If you click No, the file will not be saved and all changes will be lost. If you click Cancel, the Run or Done operation will be aborted.

The Run CONHAB button. Clicking this button causes CONHAB computations to be done. Information will be written to the screen about the files being used by CONHAB and about the progress of the computation. The user can abort the computation by pressing <Ctrl>+C. A message will be written to the screen indicating whether the computation was successfully completed. After the computation is completed the user will be asked to press <Enter> to retum to data entry for ConHAB, press $H$ to return the HABIT main window, or press $X$ to exit HABIT (return to DOS).

The Done button is used to return to the HAEIT main window. 
The Help button is used to obtain help. See using Help for more details.

10.5.1. CONHAB PIant Parameters Form.

Figure 10.3 shows this form. The following controls are used to enter plant parameters for CONHAB:

The Run Title text box.

The Control Room volume (m3 or ft3) value box.

The Reactor Power Level (MWt) value box.

The Nuclide Data File option buttons determine whether CONHAB uses the nuclide data file FGR.11, ICRP.30, or ICRP.02. See section 1.10. CONHAB uses the selected data file to obtain values of decay rates, core inventory, dose conversion factors, and daughter formation fractions.

The Group Fractions Table is used specify what fraction of each nuclide group is released in each of the three chemical forms. For instance, values of $0.95,0.04$, and 0.01 for elemental, organic, particulate in the halogens column would indicate that 95\% of the halogens released are in elemental form, $4 \%$ are in organic form, and 1\% is in particulate form.

NOTE: The reactor power level and the group fractions are used in conjunction with flows through unfiltered path 1 and unfiltered path 2 (i.e., from FPFP 2). If there is no flow of contaminants through either of these paths, then you do not need to enter values for these parameters.

The Change Units button. The currently selected units are shown with each of the variables. When the change units button is pressed a form appears that can be used to change these units. See the section on the change units form.

The Done button is used to return to the HABIT main window.

The Help button is used to obtain help. See Using Help for more details.

\subsubsection{The Change Units Form.}

The change units form, which is used for both CHEM and CONHAB, appears in Figure 10.4. It is accessible by clicking any of the change units buttons which appear throughout CHEM and CONHAB.

The top of this forms asked "Do you want numerical values converted?" If the "Yes" option button is selected, all numerical values already in the input will be converted from the old units to the new units. If the "No" option is checked, then the new units will be used for the (unchanged) numerical values.

One of two option buttons is used to choose either meters and meters** 3 or feet and feet**3 for distances and volumes respectively.

One of four option buttons is used to select the units for flow rates. Choices available are: $\mathrm{m} 3 / \mathrm{s}, \mathrm{ft} 3 / \mathrm{s}, \mathrm{m} 3 / \mathrm{min}$, and $\mathrm{ft} 3 / \mathrm{min}$. 


\subsubsection{CONHAB Meteorological or $x / Q$ Data Form.}

This form is used to enter values for the meteorological parameters that CONHAB uses to compute dilution factors $(\mathrm{X} / \mathrm{Q})$ for flows entering the control room through filtered flow paths 1 and 2. Alternately, the form can be used to input $X / Q$ values directly. See figures 10.5 and 10.6. The data on this form only need to be entered if radionuclides are assumed to flow into the control room from filtered paths 1 or 2 .

The step number value box shows the number of the current time step. A number can be typed in this box followed by <Enter> or <Tab> to move to a different time step. Typing a number beyond the last time step defined causes the program to beep and move to the last time step defined.

The step + button is used to move to the next time step.

The step - button is used to move to the previous time step.

When Compute $\mathrm{X} / \mathrm{Q}$ from Meteorology is checked, the user enters values for meteorological parameters and CONHAB computes values for $X / Q$. When Manual Entry of $X / Q$ is checked, the user directly enters values for $X / Q$. The Time drop down list. This list is used to enter new times and to review existing times. To enter a new time, simply type the time in the box at the top of this list and press either <Enter> or <Tab>. The new time will be inserted at the correct place in the sequence of time steps already in the data. To review the data for existing time steps, tab to this and use the up and down arrows to scan through the times. For more information see the section on use of drop-down lists.

When Compute $\mathrm{X} / \mathrm{Q}$ from Meteorology is checked, value boxes or option buttons appear for each of the following Meteorological Parameters:

Effluent vertical velocity

Effluent flow rate

Release height

Building height

Building area

Horizontal distance to receptor This is the distance from the point of release to the intake of the filtered flow path.

Receptor height

wind speed

The Vertical stability class option buttons are used to specify the vertical stability class $(A-G)$.

The Horizontal stability class option buttons are used to specify the horizontal stability class (A-G).

When Manual Entry of $X / Q$ is checked, a value box appears for values of 
$\mathrm{x} / \mathrm{Q}$

The Delete Time button. To delete all data for a time step, use the Time drop down list to show the time to be deleted and then press the Del Time button.

The Last time defined label shows the step number and time for the last time step that has been defined.

The Control Room Parameters button.

The Change Units button. The currently selected units are shown with each of the variables. When the change units button is pressed a form appears that can be used to change these units. See the section on the change units form.

The Done button.

The Help button. See Using Help for more details.

\subsubsection{The CONHAB Control Room Flow Parameters Form.}

This form, which is a schematic representation of the flows into the control room, is seen in Figure 10.7. It is used to enter time steps, control room flow rates and occupancy factors into CONHAB. It is identical to the CHEM Control Room Flow Parameters Form, except that filter efficiencies appear on the CONHAB form but not the CHEM form.

When entering filter efficiencies on this form bear in mind how they are used by CONHAB. The "Elemental" filter efficiency is used for the elemental form of isotopes in the halogens group. The "Organic" filter efficiency is used for the organic form of isotopes in the halogens group. The "Particulate" filter efficiency is used for the particulate form of isotopes in the halogens group and for all three chemical forms of isotopes in the sodiums, solids, and plutoniums groups. Noble gasses are not effectively removed by filters.

Items appearing on CONHAB Control Room Flow Parameters Form are:

The step number value box shows the number of the current time step. A number can be typed in this box followed by <Enter> or <Tab> to move to a different time step. Typing a number beyond the last time step defined causes the program to beep and move to the last time step defined.

The Step + button is used to move to the next time step.

The step - button is used to move to the previous time step.

The Time drop down list. This list is used to enter new times and to review existing times. To enter a new time, simply type the time in the box at the top of this list and press either <Enter> or <Tabs. The new time will be inserted at the correct place in the sequence of time steps already in the data. To review the data for existing time steps, tab to this and use the up and down arrows to scan through the times. For more information see the section on use of drop-down lists.

The Del Time button. To delete all data for a time step, use the Time drop down list to show the time to be deleted and then press the Del 
Time button.

The Last Time Defined label shows the step number and time for the last time step that has been defined.

The Unfiltered air source 1 value box is used to specify the flow of air into the control room from unfiltered air source 1 . This is used for flows through the plant to the control room as modeled by FPFP_ 2 for this flow path.

The Unfiltered air source 2 value box is used to specify the flow of air into the control room from unfiltered air source 2 . This is used for flows through the plant to the control room as modeled by FPFP 2 for this flow path.

The Bottled air release value box specifies the flow rate of bottled air into the control room.

The Filtered air source 1 frame is used to specify the flow of air into the control room from filtered air source 1 and the filter efficiencies for this filter. This is used for flows through the atmosphere to the control room.

The Filtered air source 2 (feeds recirculating filter) frame is used to specify the flow of air into the control room from filtered air source 2 and the filter efficiencies for this filter. This is used for flows through the atmosphere into ducts feeding the recirculating filter and then into the control room.

The Recirculation flow rate value box. Note that this is the rate of flow of air out of the control room into the recirculating filter. The total flow rate through the recirculating filter is the sum of this flow rate plus the flow rate into the recirculating filter from filtered air source 2 .

The Recirculating Filter frame is used to specify the filter efficiencies for the recirculating filter.

The control room occupancy Factor value box.

A label indicating the leakage rate of the control room. This shows the total rate at which air must leak from the control room to offset the inflow. It is simply the sum of the inflow rates and is provided for information purposes only.

The Met Params button may be used to switch to the Meteorological Parameters Form.

The Change Units button. The currently selected units are shown with each of the variables. When the change units button is pressed a form appears that can be used to change these units. See the section on the change units form.

The Done button.

The Help button. See Using Help for more details. 
HABIT Manual

NUREG/CR- 6210

CONHAB

\subsection{Forms for CONHAB Data Entry.}

The following three pages show forms that may be used to collect data for use in CONHAB. 
Run ID:

Run Title:

Distance and volume units:

$m$ and $m^{3}$ ft and $f t^{3}$

Flow rate units:

$\begin{aligned} & \mathrm{m}^{3} / \mathrm{s} \\ & \mathrm{ft} \mathrm{t}^{3} / \mathrm{s}\end{aligned}-\mathrm{\textrm {m } ^ { 3 } / \mathrm { min }}$

Reactor Power Level (MWt):

Control Room Volume $\left(\mathrm{m}^{3}\right.$ or $\left.f \mathrm{t}^{3}\right)$ :

Nuclide Data File:

FGR. 11 ICRP . 30 ICRP .02

Group Fractions:

$$
\text { Halogens Nobles Solids Sodiums Plutoniums }
$$

Elemental

Organic

Particulate 
Complete one page for each time step or make a table showing $\mathrm{X} / \mathrm{Q}$ for each time step.

Run ID:

Step Number: Time (hrs):

Distance and volume units:

$\mathrm{m}$ and $\mathrm{m}^{3}$ $\mathrm{ft}$ and $\mathrm{ft} \mathrm{t}^{3}$

Flow rate units: $\mathrm{m}^{3} / \mathrm{s}$ $\mathrm{m}^{3} / \min$ $f t^{3} / s$ $\mathrm{ft} \mathrm{t}^{3} / \mathrm{min}$

Meteorological Parameters:

Effluent vertical velocity $(\mathrm{m} / \mathrm{s})$

Effluent flow rate (flow rate units)

Release height ( $\mathrm{m}$ or $\mathrm{ft}$ )

Building height ( $m$ or $f t$ )

Building area $\left(\mathrm{m}^{2}\right.$ or $\left.\mathrm{ft}^{2}\right)$

Horizontal distance to receptor (m or $f t$ )

Receptor height ( $m$ or $f t$ )

Wind speed $(\mathrm{m} / \mathrm{s})$

Vertical stability

Horizontal stability

$\begin{array}{lllllll}A & B & C & D & E & F & G\end{array}$

$\begin{array}{lllllll}A & B & C & D & E & F & G\end{array}$ 
HABIT Manual

NUREG/CR - 6210

CONHAB

CONHAB Work Sheet

Control Room Flow Parameters

Complete one page for each time step.

Run ID:

Step Number:

Time (hrs):

Flow Parameters:

(Flow rate units for flows and percent for filter efficiencies.)

Flow from unfiltered source 1 (FPFP_2 A)

Flow from unfiltered source 2 (FPFP_2 B)

Bottled air release flow

Flow from filtered source 1 (TACT5 A)

Filter efficiencies

Elemental Organic Particulate

Flow from filtered source 2

(TACT5 B, feeds recirc.)

Filter efficiencies

Elemental Organic Particulate

Flow from control room

into recirculating filter

Recirculating filter efficiencies

Elemental Organic Particulate

Occupancy factor (fraction) 


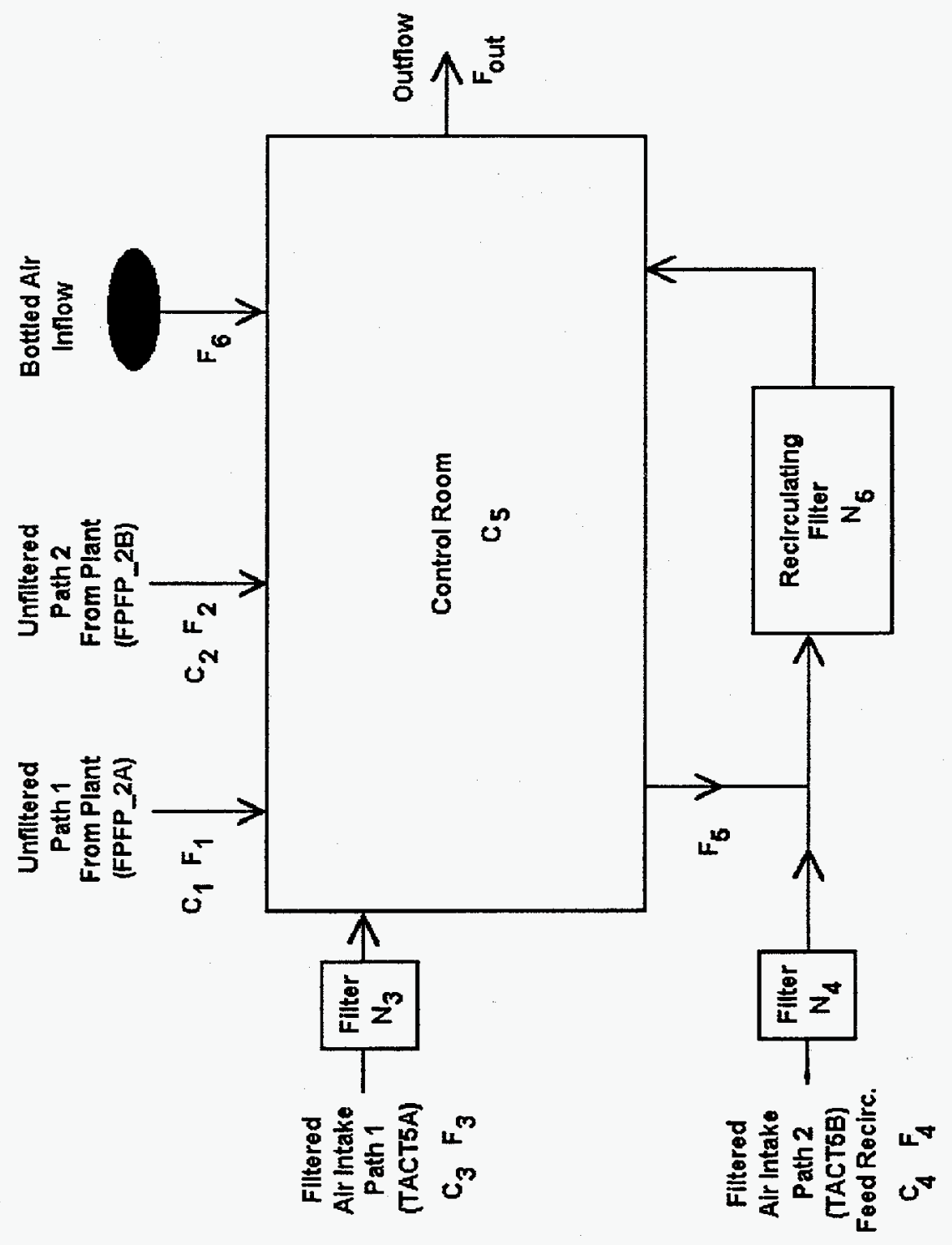

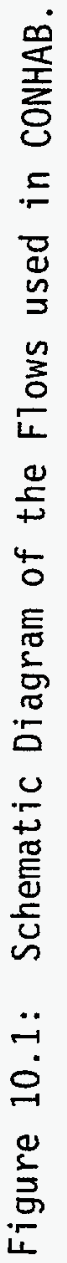




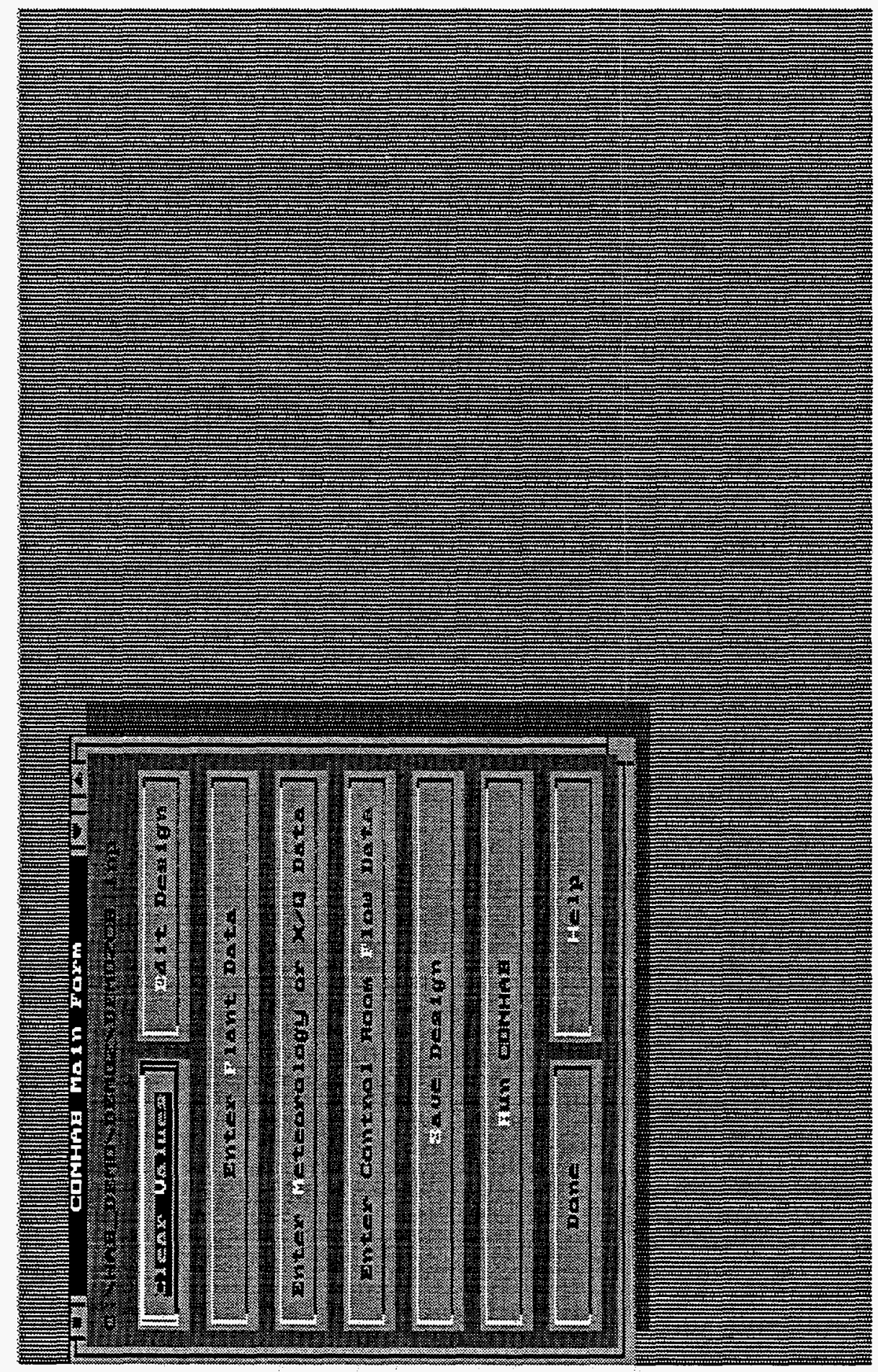

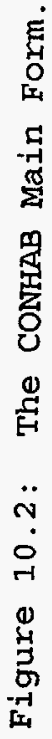




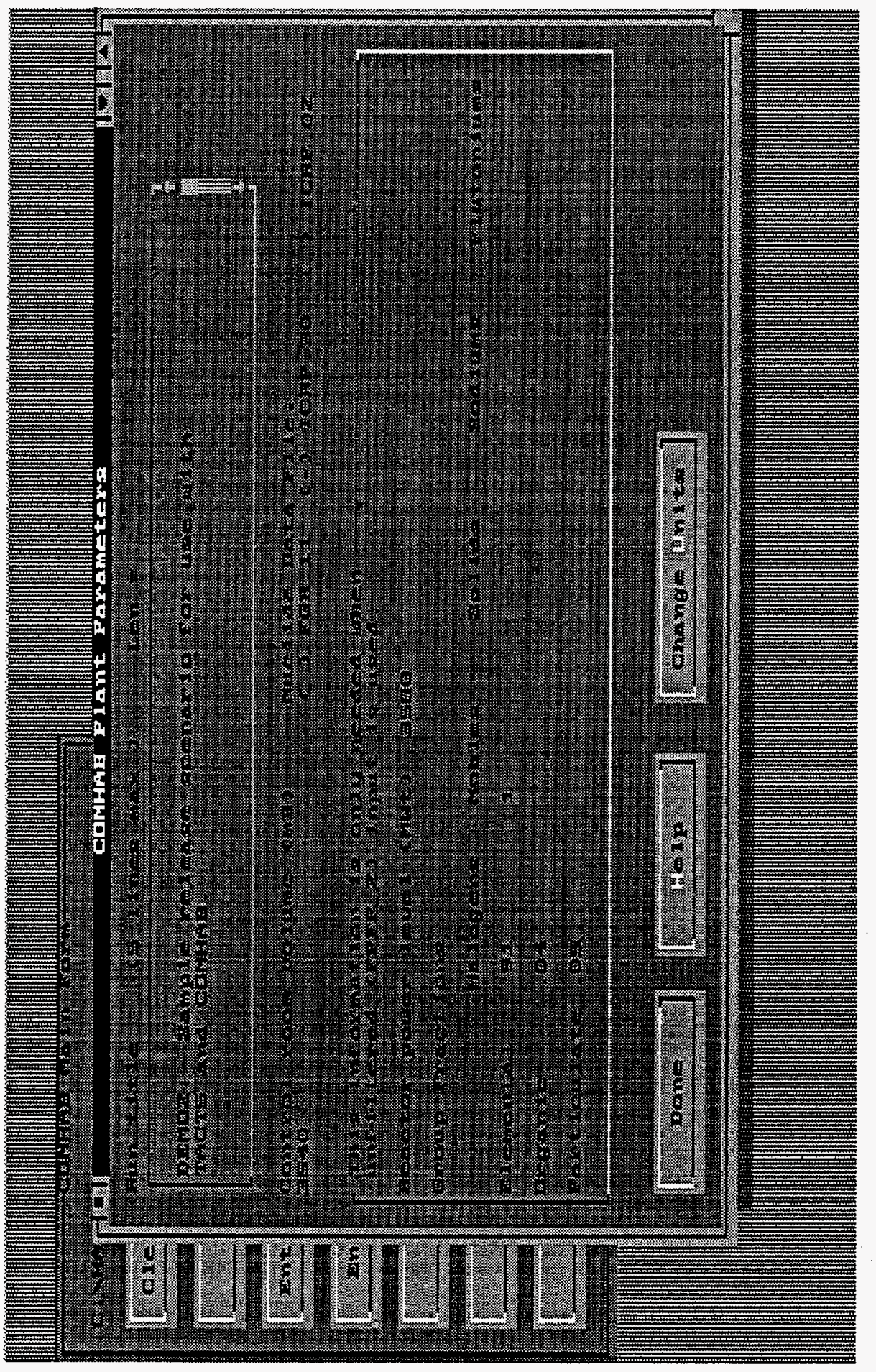

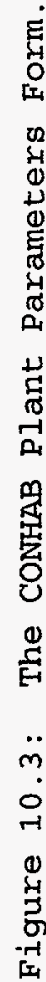




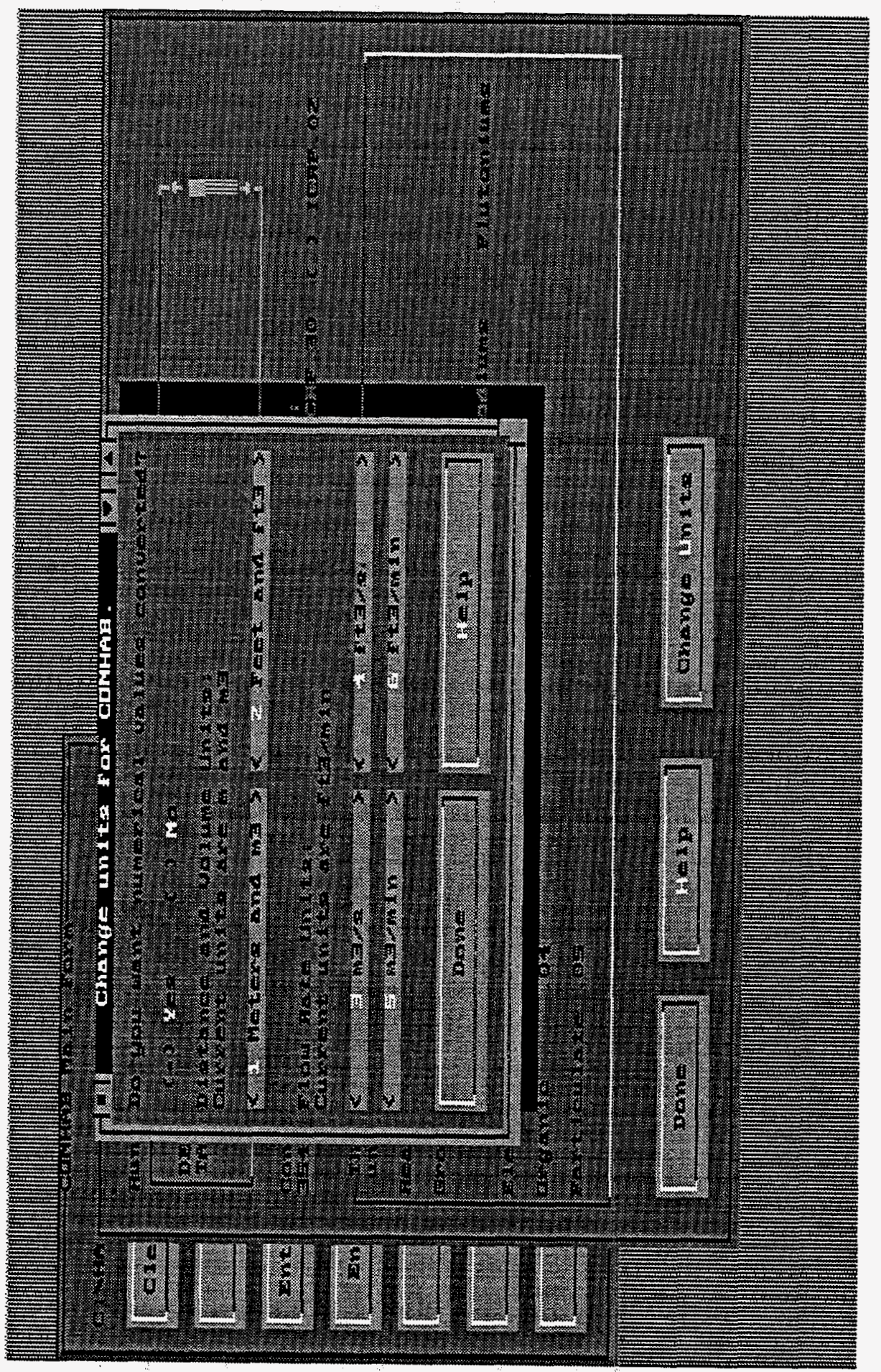

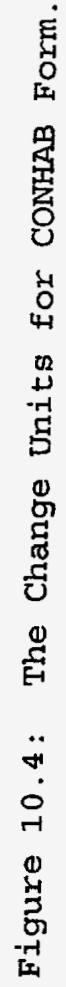




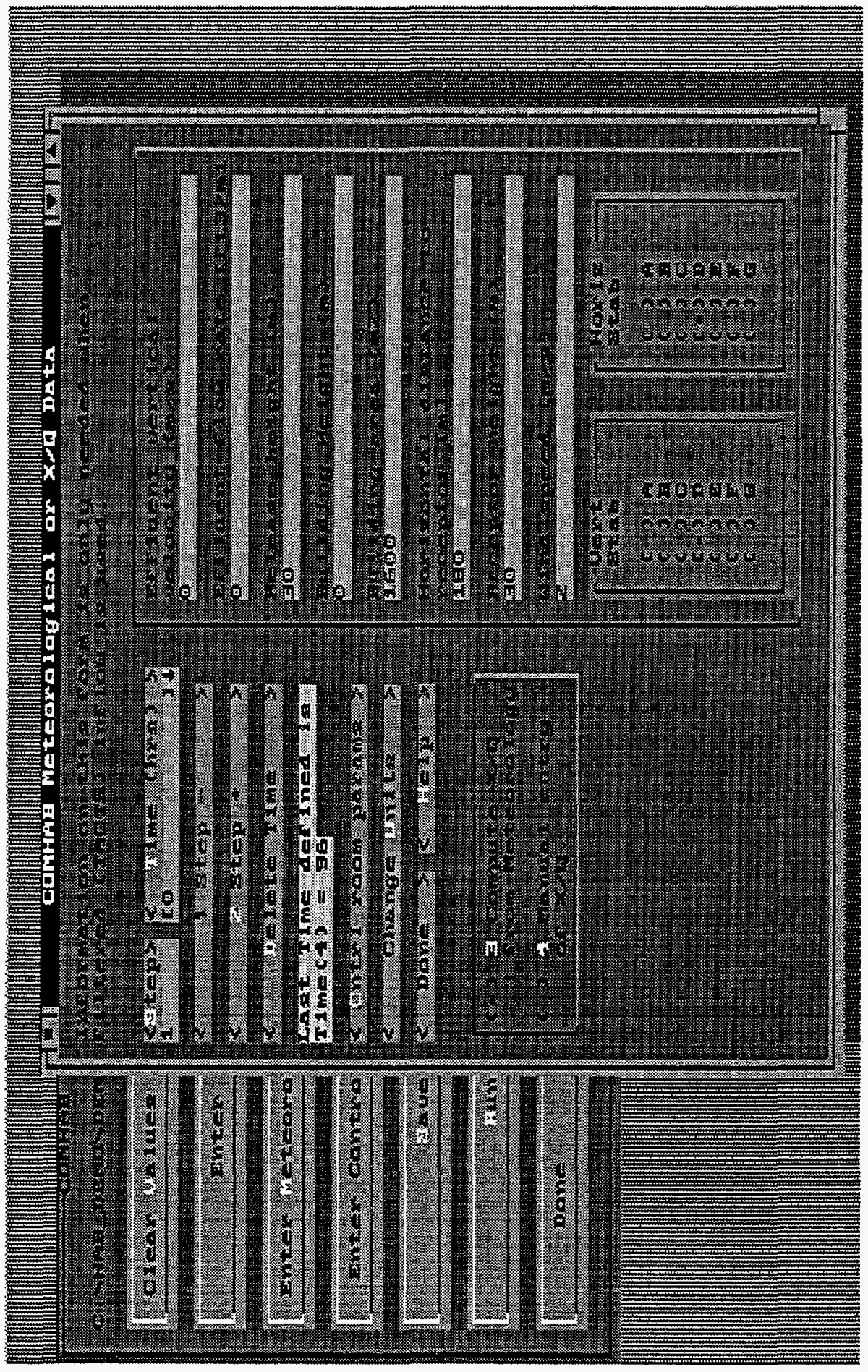

庭

苗

\%

Q

丽

मे

겅

的

of

감

우

过

口

包苾

ต

受

궁웅

옹

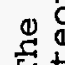

崖

in 5

엇

告

[is 


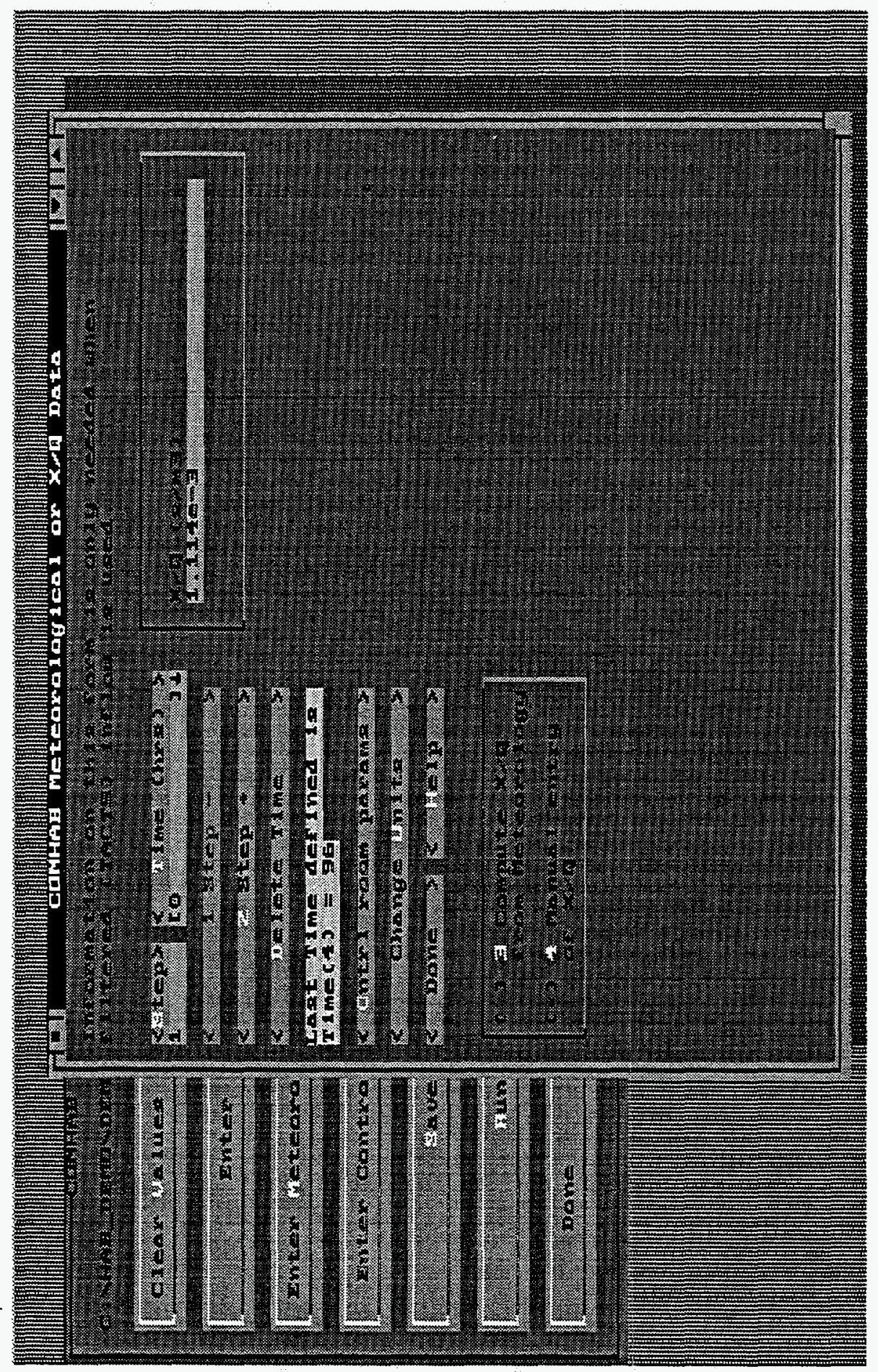

్ㅗㅇ

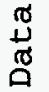

Or

엉

겅

0

每目

O.

이

भै

O

㐘

音

존

잉

点峦

$\ddot{0}$

$\ddot{6}$

$\stackrel{0}{\rightarrow}$

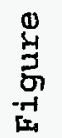




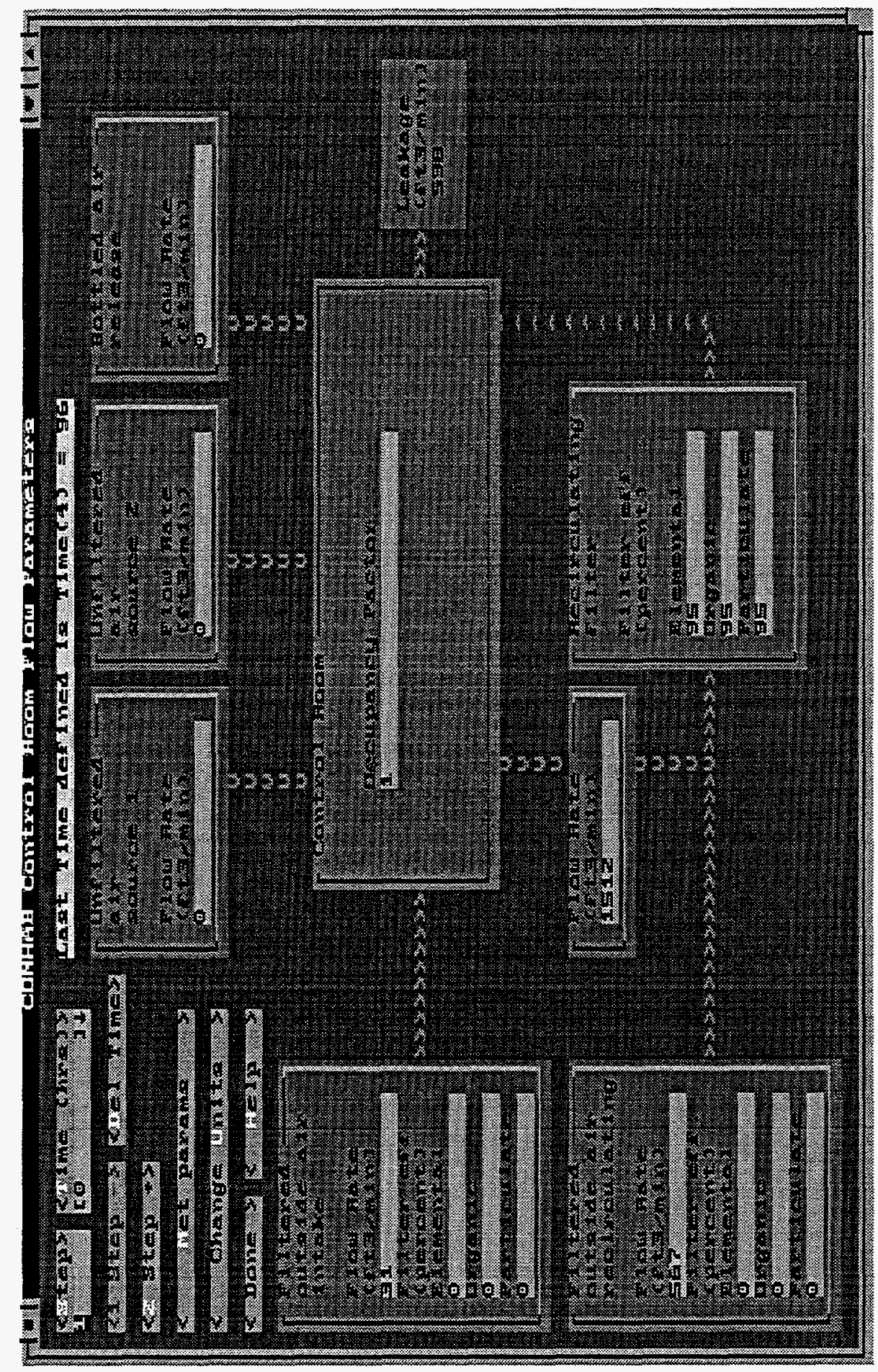

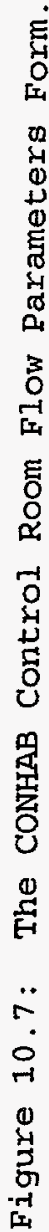



Three runs of HABIT are included in the HABIT distribution package and may be installed when you install HABIT. They are in the directories $C: \backslash H A B$ DEMO \DEMO1, C: \HAB_DEMO \DEMO2, and $C: \backslash H A B$ DEMO\DEMO3. These may be used to familiarize yoursēelf with the use of HABIT and to verify that your version of HABIT gives the correct answers.

DEMO1 is a design for running EXTRAN and CHEM for a hypothetical toxic chemical release. DEMO2 is a design for running TACT5 and CONHAB for a hypothetical radioactive release. DEMO3 is a design for running FPFP_2 and CONHAB for a hypothetical radioactive release. The scenarios for these releases are briefly discussed below. All design specification (input) files needed to run HABIT are included as are all of the files produced by HABIT. The output files, *.TAB, and *.SPD are designed to be self-explanatory. Some of the program output files have been shortened to conserve space on the distribution disks. places where data has been removed are indicated in the files.

A good way to familiarize yourself with the operation of HABIT is to start HABIT, click the "Edit Design" button, then click the "Copy Design" button. COpY DEMO1, DEMO2, or DEMO3 to another path and design name. You may then experiment using the copy to review the designs, make changes and run the HABIT programs.

\subsection{DEMO1, A Hypothetical Toxic Chemical Release.}

DEMO1 is a hypothetical release of chlorine gas due to the rupture of a tank car. This release is shown schematically in Figure 11.1. In the demonstration run, contaminated air enters the control room for 30 seconds following the arrival of the plume. The control room is then isolated by shutting the external intake and using bottled air.

Figure 11.2 shows chlorine concentration versus time for the air at the control room intake and in the control room for the cases with and without isolation using bottled air. This plot was produced from the DEMO1EX.SPD and DEMO1CH.SPD files by importing them into Quatro Pro as quote and comma delimited files. Making the run with no isolation is left as an exercise for the reader.

The horizontal line across the middle of Figure 11.2 shows the current NRC exposure limit of 30 ppm for chlorine gas as proposed by Mahlum and Sasser (1991, p. 15). It can be seen that the isolated control room remains below the exposure limit in this case.

Even without isolation, the control room concentration is less than the intake concentration during the first few minutes due to the time delay associated with ventilation of the control room. This delay provides some time for the control room to be isolated. Control room concentrations fall off exponentially once the control room is isolated. 
HABIT Manual

NUREG/CR- 6210

Sample Runs

Another exercise which may be done by the reader is to make a sequence of EXTRAN runs for distances of $50,100,200,500$, and 1000 meters between the spill and the control room intake keeping other parameters the same. Values of average concentration during the first two minutes after plume arrival can then be plotted as a function of distance. One can also plot maximum concentration during the first two minutes after plume arrival versus distance. 
HABIT Manual

NUREG/CR - 6210

Sample Runs

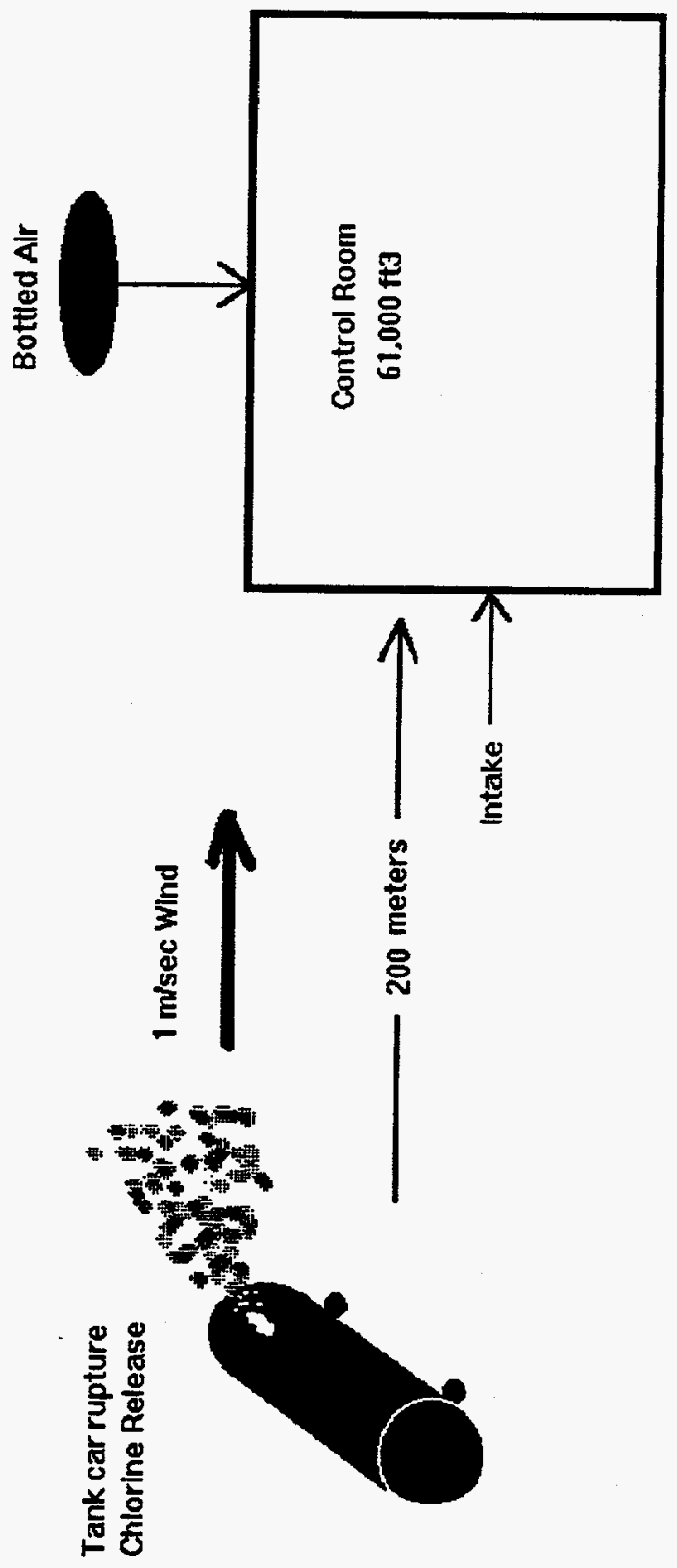

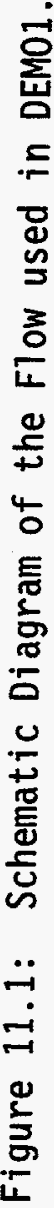




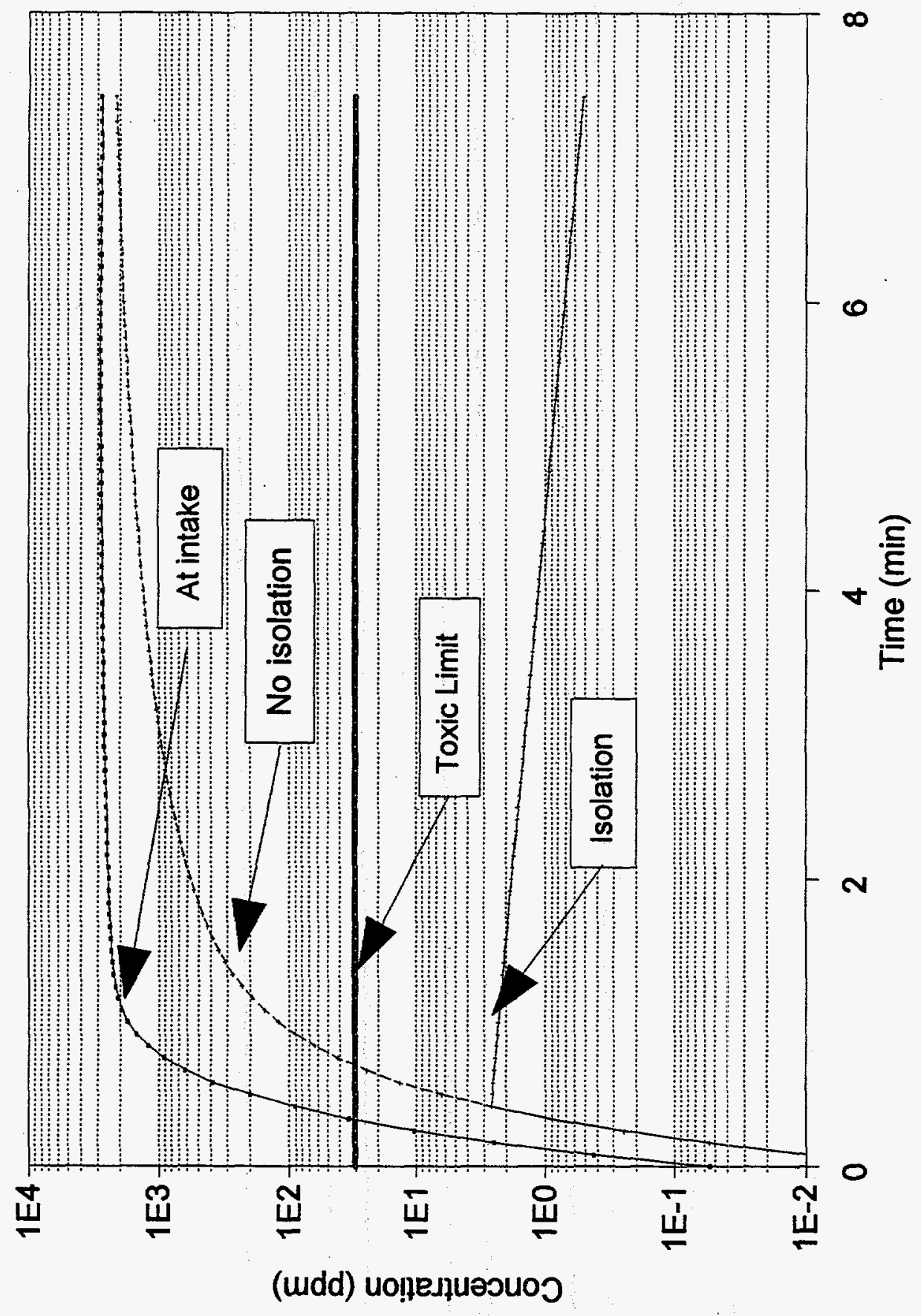

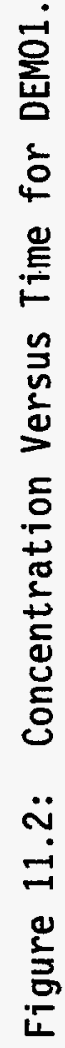


The DEMO2 example is a hypothetical release of radioactive halogens and noble gasses to the atmosphere, followed by intake of the radioactivity into the control room. The release rates to the atmosphere are computed by TACT5 and the subsequent control room doses are computed by CONHAB. This case is a simulation of a loss of coolant accident (LOCA). This case is shown schematically in Figure 11.3. TACT5 is used to model the containment and to determine the rate of nuclide release to the atmosphere. Once the nuclides are released to the atmosphere, CONHAB takes over the computation. CONHAB computes values for $X / Q$, uses these to determine concentrations at the control room intakes (filtered paths 1 and 2), and then determines control room concentrations and the associated doses.

Figure 11.4 is a schematic representation of the way that the containment is modeled by TACT5. The containment building in this case is equipped with sprays. However, the operating floor and other structures prevent the sprays from effectively reaching all parts of the containment. For this reason, the containment has been modeled as two nodes: "spray" and "unspray" with unfiltered transfer representing convection between the two.

The accident simulation starts with a puff release into the spray and unspray nodes. During the entire scenario, activity leaks from the containment to the environment. This leak is modeled as an unfiltered transfer. From 672 to 675 hours and again from 696 to 699 hours, the containment is vented to the atmosphere through filters.

Air flows into the control room used by CONHAB in this case are shown in Figure 11.5. Using the notation of Chapter 10, we have

$$
\begin{array}{lr}
F_{1}= & 0 \\
F_{2}= & 0 \\
F_{3}= & 31 \\
F_{4}= & 567 \\
F_{5}= & 1512 \\
F_{6}= & 0 \\
N_{3}= & 0 \\
N_{4}= & 0 \\
N_{5}= & 0.95 \\
C_{3}=C_{4}
\end{array}
$$

The equations in section 10.2 then become

$$
\begin{aligned}
R_{\text {in }} & =C_{1} F_{1}+C_{2} F_{2}+C_{3} F_{3}\left(1-N_{3}\right)+C_{4} F_{4}\left(1-N_{4}\right)\left(1-N_{5}\right) \\
& =C_{3}\left(F_{3}+F_{4}\left(1-N_{5}\right)\right) .
\end{aligned} \quad \begin{aligned}
F_{\text {out }} & =F_{1}+F_{2}+F_{3}+F_{4}+F_{6} \\
& =F_{3}+F_{4} .
\end{aligned}
$$


HABIT Manual

NUREG/CR- 6210

Sample Runs

$$
\begin{aligned}
R_{\text {out }} & =F_{\text {out }}(A(t) / V)+F_{5} N_{5} A(t) / V \\
& =\left(F_{\text {out }}+F_{5} N_{5}\right) A(t) / V \\
& =\left(F_{3}+F_{4}+F_{5} N_{5}\right) A(t) / V \\
& =\left(F_{3}+F_{4}+F_{5} N_{5}\right) C_{C r}(t)
\end{aligned}
$$

where $\mathrm{C}_{\mathrm{Cr}}(t)$ is the control room concentration at time $t$. After a long time, a steady state is reached in the control room when the rates of activity entering and leaving the control room are equal $\left(\mathrm{R}_{\text {in }}=\mathrm{R}_{\text {out }}\right)$. At that time

$$
C_{C r} / C_{3}=\left(F_{3}+F_{4}\left(1-N_{5}\right)\right) /\left(F_{3}+F_{4}+F_{5} N_{5}\right)
$$

This leads to the definition of the iodine protection factor (IPF) as

$$
I P F=\left(F_{3}+F_{4}+F_{5} N_{5}\right) /\left(F_{3}+F_{4}\left(1-N_{5}\right)\right)
$$

According to a 1974 paper by Murphy and Campe the inhalation dose within the control room can be estimated by the inhalation dose at the control room intake divided by the IPF.

TACT5 estimates the thyroid dose outside the control room as 1911 rem (see the file DEMO2T5A.TAB). For this case IPF = 34.2. As discussed on page 6.4-11 of the Standard Review Plan, the estimate of the dose in the control room can be further reduced by a factor of 10 if the plant has two widely-separated air intakes. The Murphy-Campe method therefore produces an estimate of $5.59 \mathrm{rem}$ thyroid dose in the control room.

According to CONHAB, the control room thyroid dose (see the file DEMO2CB.TAB) is 56.49 rem. When this is reduced by the factor of 10 as a credit for dual air intakes, the control room dose becomes 5.65 rem.

In this case there is very close agreement between the doses found using the Murphy-Campe method and those found by CONHAB. For this case the protection afforded by the time delay of control room ventilation is nearly exactly offset by the increase in dose associated with daughter formation. See the discussion in the chapter on CONHAB.

Figure 11.6 shows the thyroid rem/hr at the intake and in the control room. The curve for the ${ }_{3}$ ir intake was computed using CONHAB with a small control room volume $\left(10 \mathrm{~m}^{3}\right)$ and zero values for filter efficiencies. This computation can be done by the reader as an exercise. The thyroid dose computed by CONHAB for the intake was $1970 \mathrm{rem}$. The difference between this value and the value of 1911 computed by TACT5 is daughter formation. Thus we see that daughter formation increased the thyroid dose in this case by about $3 \%$. Taking the intake dose computed by CONHAB (1970 rem), dividing by the IPF (34.2), and comparing the answer with the control room dose computed by CONHAB $(56.49)$ shows that the time delay associated with control room ventilation decreases the thyroid dose in this case by about $2 \%$.

The protecting influence of the time delay of control room ventilation can be seen for early times in Figure 11.6. Another feature in this case is the sudden drop in rem/hr at the intake at 8 and 24 hours due to changes in $X / Q$ in the release scenario. These changes are associated with assumptions made in determining 8 hour and 24 hour $\mathrm{X} / \mathrm{Q}$ values for regulatory purposes and not with any sudden changes that would occur in the real atmosphere. This produces control room concentrations that are greater than those outside. In an actual 
HABIT Manual

NUREG/CR-6210

Sample Runs

situation, control room staff could reduce control room doses by ventilating the control room during periods when intake concentrations are less than control room concentrations. 
HABIT Manual

NUREG/CR- 6210

Sample Runs

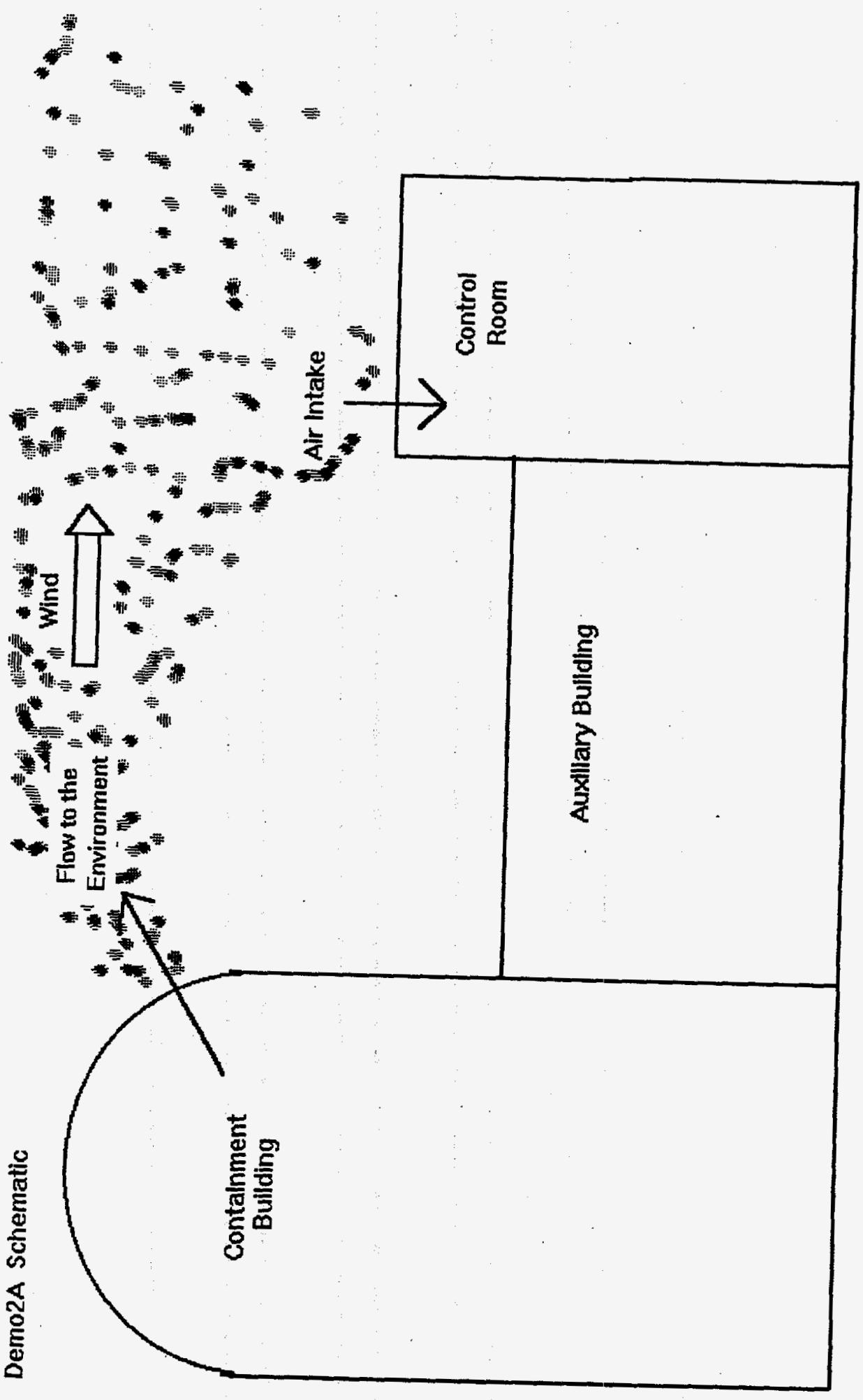

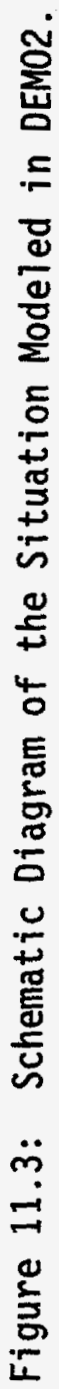


HABIT Manual

NUREG/CR - 6210

Sample Runs

Demo2 Schematic

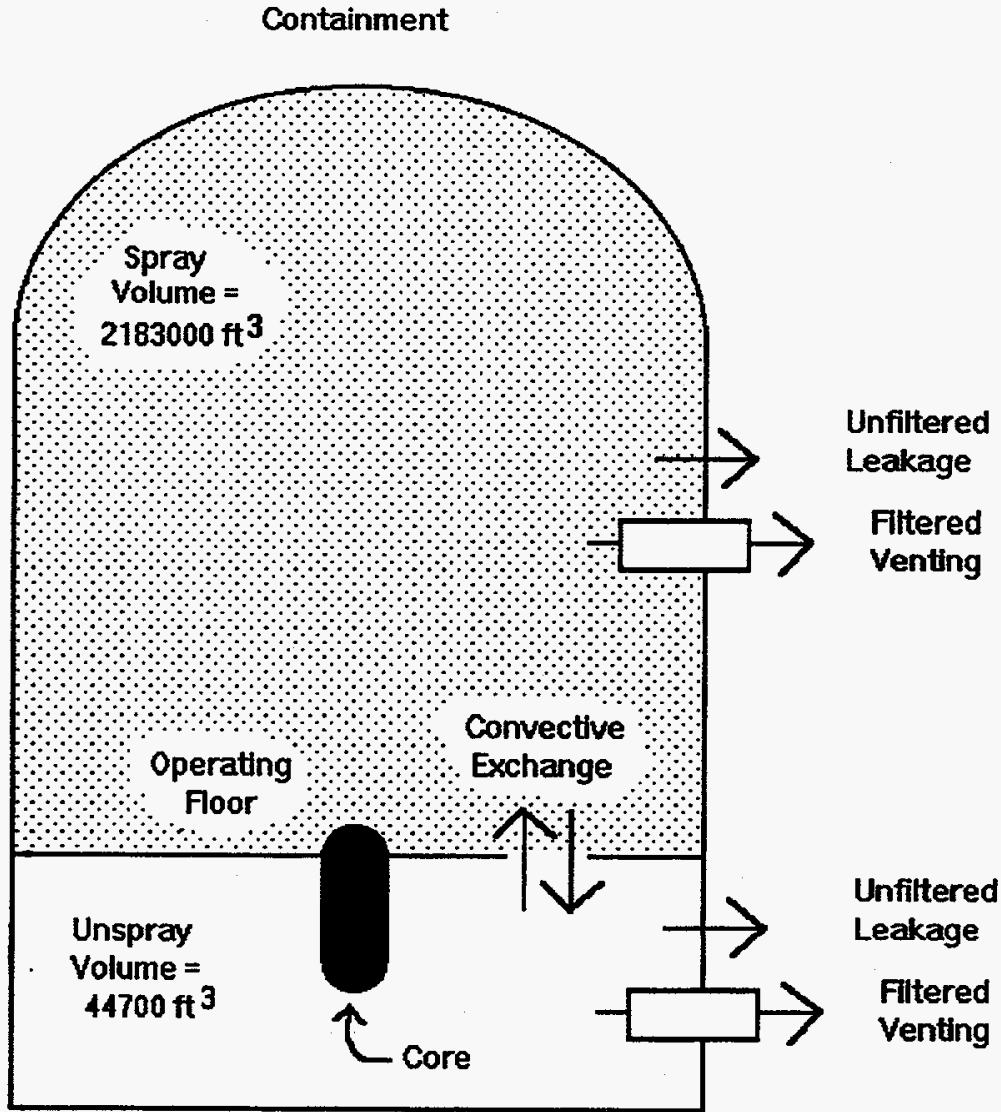

Figure 11.4: Schematic Diagram of the Containment Flows Used in TACT5 for DEMO2. 
Demo2, Control Room Flows

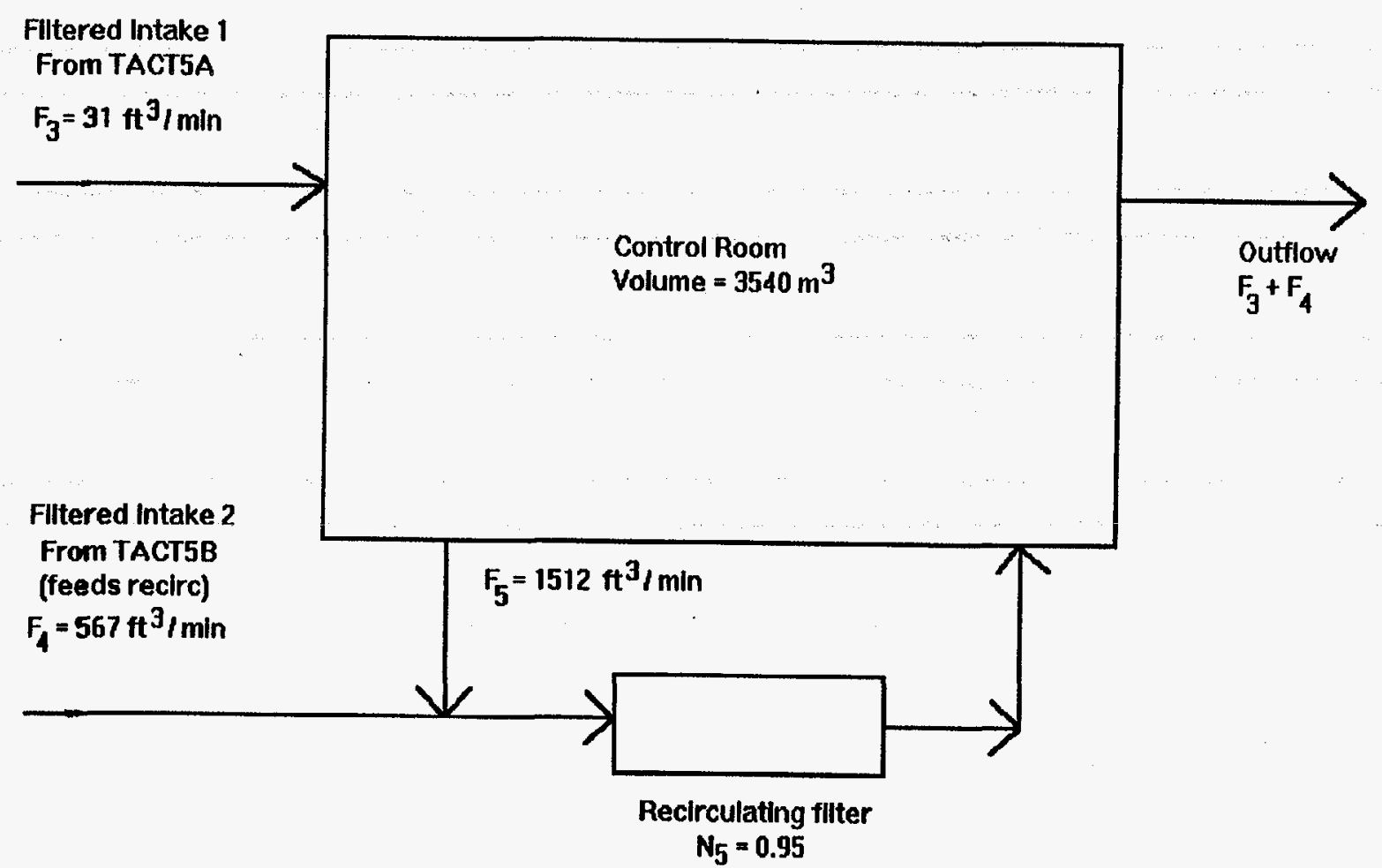

Figure 11.5: Schematic Diagram Control Room Flows Used in CONHAB for DEMO2. 


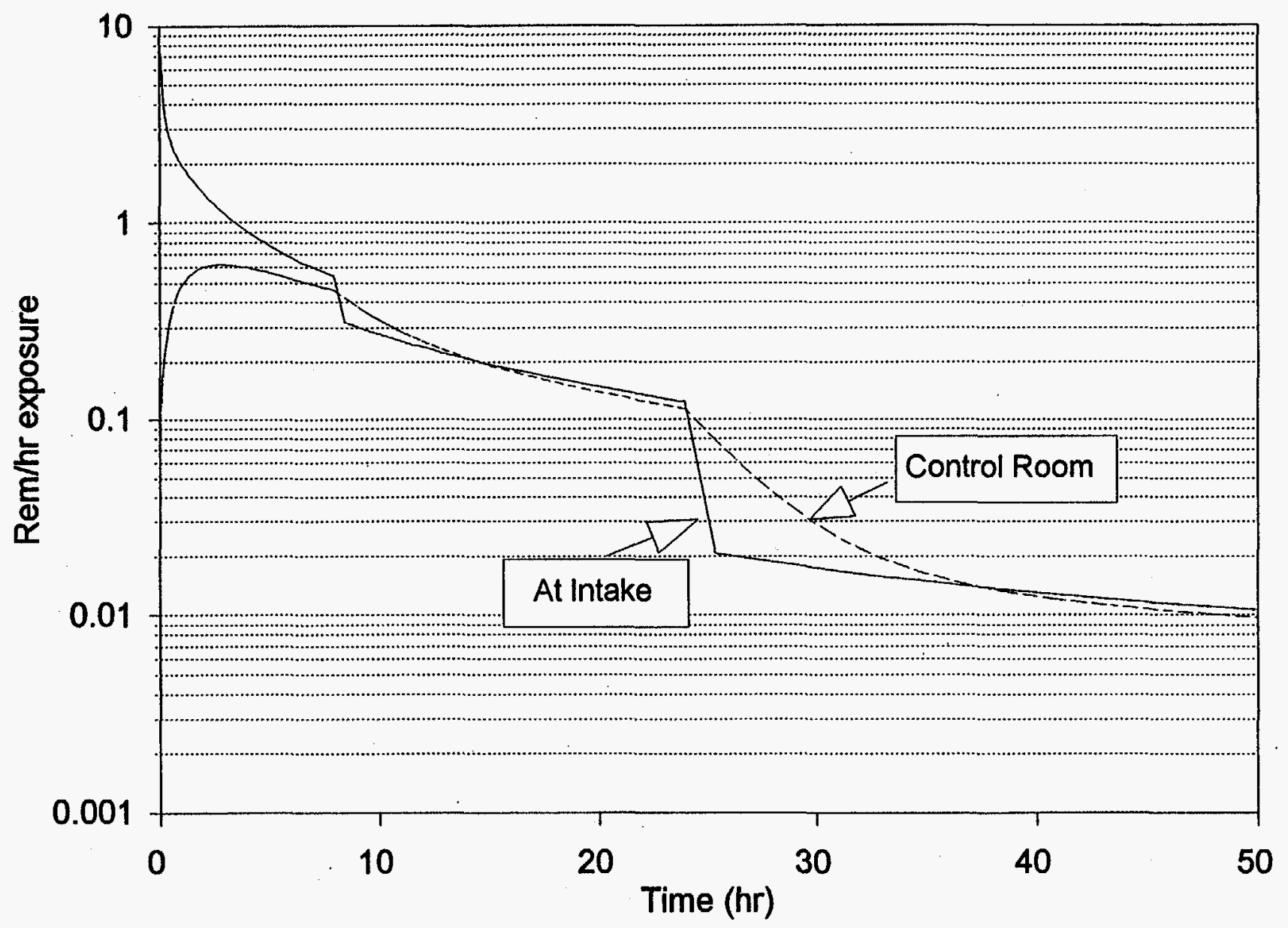

Figure 11.6: Rem/hr Exposure within the Control Room and at the Intake for DEM02. 
DEMO3 is a hypothetical scenario for the release of radioactivity from the core into the containment and its subsequent flow through the plant into the control room. Figure 11.7 gives a schematic diagram of this situation. The way that this case in modeled by FPFP_2 is shown schematically in Figure 11.8 .

In this caşe the containment volume is $21600 \mathrm{ft}^{3}$ and the control room volume is $6250 \mathrm{ft}^{3}$. One percent of the core is released into the containment at time 0 . This gives puff release concentrations of

$$
0.01 \text { core fraction } / 21600 \mathrm{ft}^{3}=4.6 \mathrm{E}-8 \text { core fraction } / \mathrm{ft}^{3} \text {. }
$$

The containment is ventilated with 1 air change per hour giving a flow rate of

$$
21600 \mathrm{ft}^{3} / 60 \mathrm{~min}=360 \mathrm{ft}^{3} / \mathrm{min}
$$

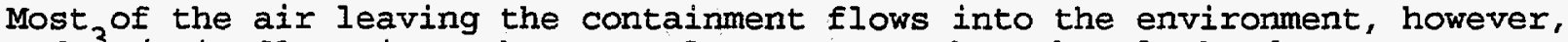
$4 \mathrm{ft}^{3} / \mathrm{min}$ flows into the control room, say through a leaky door. As seen in Figure 11.8, this situation is modeled in FPFP_2 using a resistance type space to split the flow. The control room is ventiläted with $375 \mathrm{ft}^{3} / \mathrm{min}$ of clean external air. 


\section{Fig for $\mathrm{DEMO3}$}

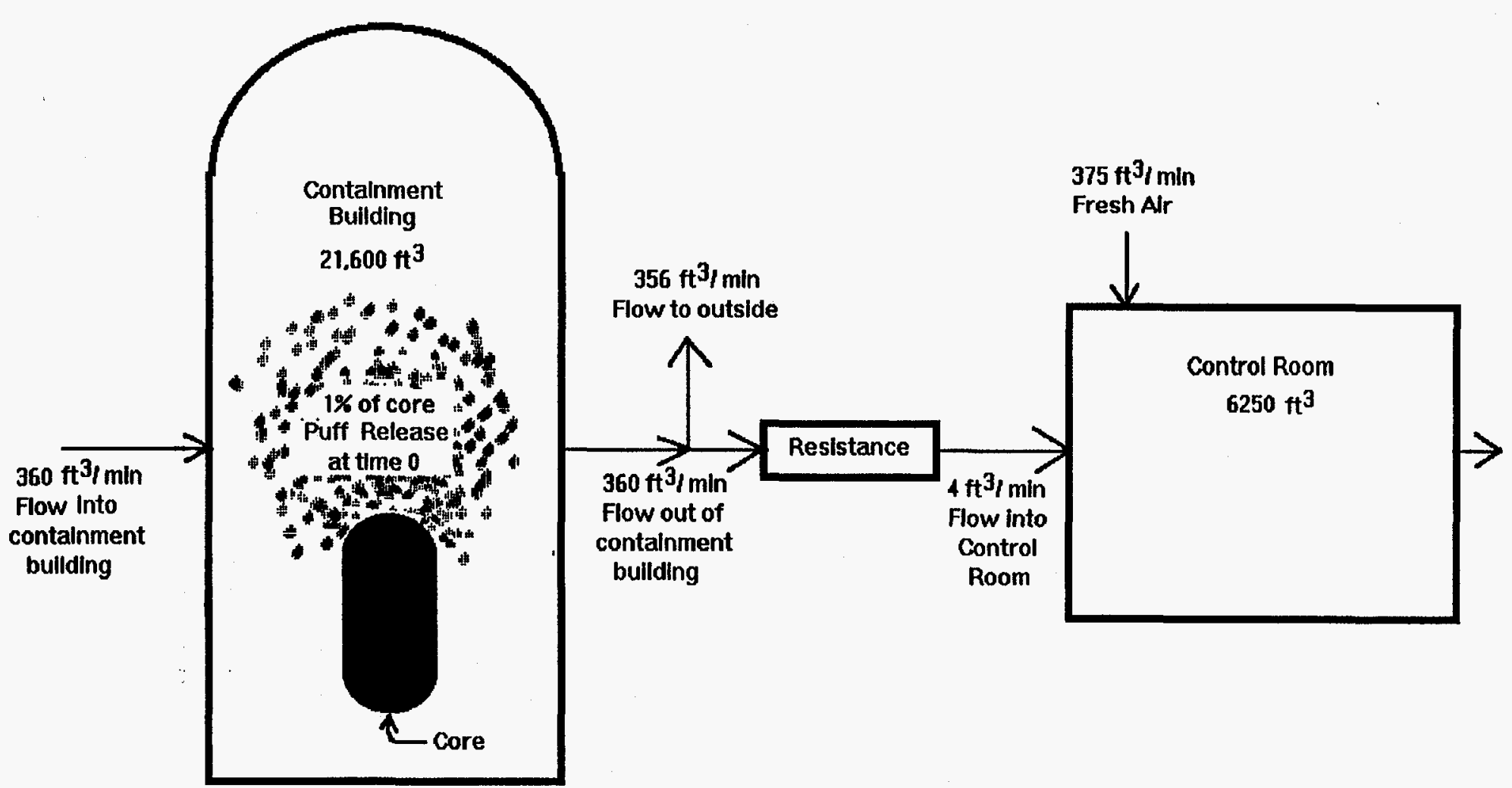

Figure 11.7: Schematic Diagram of the Situation Modeled in DEM03. 
HABIT Manual

NUREG/CR - 6210

Sample Runs

Fig for DEMO3A

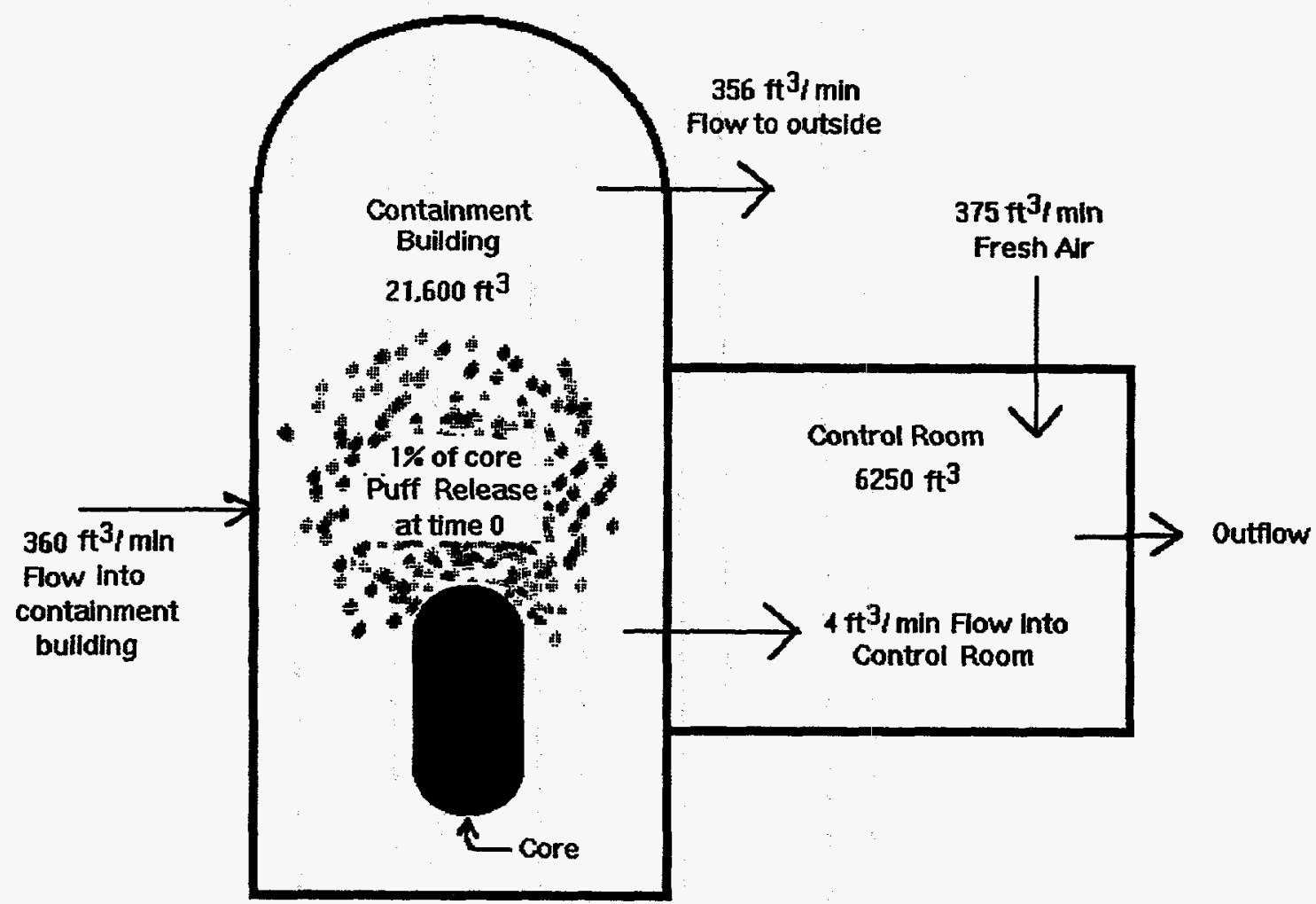

Figure 11.8: Schematic Diagram of the way DEMO3 is Modeled by FPFP 2 . 
This chapter is intended for use by persons who need to gain a deeper understanding of the programming details of the HABIT programs. It contains a number of informal comments about the programs. Most users can skip this chapter without missing any information needed to run HABIT.

The distribution disks for HABIT contain all files needed to run HABIT on a personal computer running DOS 5.0 or higher as well as files for demonstration runs of the programs. The disks also contain source code for the HABIT data entry and computational routines.

The source code for HABIT consists of nearly 35,000 lines of code and comments in 110 files. Nearly 33,000 lines of the code and comments are Visual Basic for DOS routines for data entry. The remainder are the Fortran computational routines.

The programs have been designed to smoothly move from one module to the other as needed.

\subsection{HABIT Executable Modules.}

The executabie files in the HABIT package can be divided into two groups. The first group consists of programs used for data entry and which control the flow of execution. These where written in Microsoft Visual Basic. They are:

XHABIT.EXE, the habit main window.

XEXTRAN. EXE, data entry for EXTRAN

XCHEM.EXE, data entry for CHEM

XFPFP2.EXE, data entry for FPFP_2

XCONHAB. EXE, data entry for CONHAB

XTACT5.EXE, data entry for TACT5, everything not in the next two modules.

QTACT5.EXE, data entry for TACT5 nuclide selection

STACT5.EXE, data entry for TACT5 time dependent variables

RUNPROG. EXE, RUNPROG2 . EXE, used by data entry routines to shell computational routines (see below).

VBLINK.EXE, not actually used when running HABIT. This routine is used when linking the HABIT routines as explained below. 
HABIT Manual

NUREG/CR- 6210

Programming Notes

The second group of executable files consists of the computational routines. These were written in Microsoft Fortran. They are:

EXTRAN.EXE, the computational module for EXTRAN

CHEM.EXE, the computational module for CHEM

FPFP_2.EXE, the computational module for FPFP_2

CONHAB.EXE, the computational module for CONHAB

TACT5.EXE, the computational module for TACT5

\subsection{HABIT Data Entry Routines.}

The data entry routines have all been written especially for HABIT and are new in this version. They are written in Microsoft Visual Basic for Dos, Version 1.0. The source codes for the programs contain fairly extensive in-line comments and should be understandable by anyone familiar with Visual Basic. The comments here are intended to provide additional assistance concerning a few aspects of the code.

Most of the data entry routines are too large to be linked while in the Visual Basic Editor. The file HABIT.MAK contains all of the dependencies needed for compiling and linking the HABIT data entry routines. It is intended for use with the NMAKE utility that comes with Microsoft Fortran and with the IIB library program that comes with both Visual Basic for Dos and Microsoft Fortran.

Sometimes the command lines needed to link the data entry routines were too long. This problem was surmounted by use of a special utility VBIINK. EXE that was written for use with HABIT.MAK.

WARNING: When linking Visual Basic Routines it is important to use the IINKER that comes with Visual Basic and NOT any other linker. If you use an unsuitable linker the code may appear to compile, but will not execute.

The file HABIT.MAK will be extremely useful for learning how the many forms and files used in the HABIT data entry routines fit together.

The files QTACT5.MAK, RUNPROG.MAK, RUNPROG2.MAK, STACT5.MAK, XCHEM.MAK, XCONHAB.MAK, XEXTRAN.MAK, XFPFP2.MAK, XHABIT.MAK, and XTACT5.MAK are NOT true makefiles, that is they do not show dependencies for use by NMAKE. These files are project files for use by the Visual Basic programming Environment.

The files ALLBAS.MAK, ALLFRMS.MAK, ALLFRMS1.MAK, ALLFRMS2.MAK, ALLFRMS3.MAK, AILFRMS4.MAK, AIIMAIN.MAK are project files for use in the Visual Basic Programming Environment. They can be used when you want to do some search or edit process on all of the HABIT data entry routines.

The file C: \HABIT\SPECFILE is used as a template for creating design files (ID--.DSG). It is read and all occurrences of "DEMO-" are replaced with "ID---". This file and only this file establishes the naming conventions used by HABIT.

The file $C: \backslash H A B I T \backslash S P E C D E S I$ is used tell HABIT routines which design file is currently being used. It contains the path to the current design and the name 
of the design specification file.

HABIT.BAT sets the DOS environmental variable EXEPATH to "C:\HABIT\". AII HABIT routines use this environment variable when shelling programs and when looking for files expected to be on C: \HABIT (such as SPECFILE, ICRP.30, and RANGES.SET) .

The DOS environment variable DEBUG has been used to turn on/off various features of the program that were useful while debugging the code. In the current version setting DEBUG=YES results in the program asking if it is okay to proceed before shelling a different module.

When a button is pressed to run one of the computational routines, the data entry program transfers control to the program RUNPROG.EXE. RUNPROG.EXE reads the file ID-...DSG and creates the file ID---xxx.RUN, where $\mathrm{xxx}$ is $\mathrm{EX}, \mathrm{CH}$, T5A, T5B, FPA, FPB, or CB depending on which program is to be run. ID-- XXX. RUN contains the names of the input and output files that will used by the computational routines. RUNPROG.EXE then transfers control to RUNPROG2.EXE, which shells the computational program. When the computational program is finished, RUNPROG2. EXE returns control to the data entry program, the HABIT Main Window, or DOS, depending on the key pressed by the user. RUNPROG.EXE and RUNPROG2.EXE are used in order to maximize the amount of memory available to the computational routines. Some of the computational routines are too big to run if they are shelled directly from the data entry routines or even if shelled from RUNPROG. EXE.

The routines in MSGFRM.BAS and MSGFORM.FRM are not currently used by the HABIT data entry routines. They were written to provide an easy way to obtain output on the screen during program development and have been left with the HABIT source codes so that they may be used during future work.

\subsection{The HABIT Computational Routines.}

The FORTRAN routines that perform the actual HABIT computations are documented in the NUREG's and NUREG/CR's that have been referenced above for each of the routines. Some of those references contain discussion of the program components that may be useful if you need more details than are given here.

The original Fortran codes varied widely in programming style and in legibility. Some parts of the code have been entirely rewritten, some have had major editing, and some have had little or no changes. As a result the legibility of the code and the completeness of the in-line comments is uneven. Whenever routines were modified, changes where made to make them more readable. Unfortunately there was neither time nor money in the HABIT development task to allow editing all of the source code to make it fully legible or to bring it up to modern standards of program formatting.

The HABIT package was developed using the Microsoft Fortran compiler, Version 5.10. Although most of the code should be portable to other Fortran 77 compilers, a couple of non-portable features have been used.

The most important is that the code contains in-line comments separated from the Fortran code by exclamation marks (!). It is also important that the READ (iou, ${ }^{*}$ ) statements be able to ignore items following exclamation marks. This feature is used to place comments in the *.INP files.

The *.INP files written by the HABIT data entry routines contain comments explaining the meaning of variables. 
HABIT Manual

NUREG/CR- 6210

Programing Notes

exclamation marks (1). These comments are considered to be sufficient to allow a user to understand the contents of these files without need for further documentation.

Both *.INP and *.CNX files are read using * format. The programs are therefore able to read these files even if the number of columns or location of decimal points in the numbers is changed. The one exception is that nuclide concentrations listed in the files ID---T5A.CNX and ID---T5B.CNX must be formatted using

$$
\text { FORMAT (1X, A8, 1X, I5, 1P, 2E11.3) }
$$

The computational modules in HABIT can be compiled from their Fortran source files using the makefiles: CHEM.MAK, CONHAB.MAK, EXTRAN.MAK, FPFP_2.MAK, and TACT5 . MAK. 
This section contains a description of how TACT5, FPFP 2 and CONHAB determine the size of computational time steps. The issue of time steps arises because earlier versions of FPFP 2 and CONHAB used the time steps provided by the user, which could be too large resulting in very poor computational accuracy.

Simple tests indicate that the rules stated below enable CONHAB to produce accurate dose estimates even when the user enters time steps much longer than the time scale over which control room concentrations change. Nonetheless, the user should think about the size of the time steps specified in the release design. The user should consider the air exchange interval (i.e., the inverse of the number of air changes per hour) of the control room and the half lives of the nuclides that most strongly contribute to the doses. The user should specify time steps during the early part of the release that are not more than 5 or 10 times the air exchange interval and not more than 5 or 10 times the half lives of the most significant nuclides. This helps to ensure that the program doses not miss quickly evolving changes in dose rate at the start of the episode. Longer time steps can be taken later when the short-lived nuclides have decayed away and when the control room has been ventilated by several air changes.

The algorithm used by the programs to select time steps is an attempt to ensure that the time steps are small enough to produce small computational errors (less than 5\%) even if the user specifies long time steps. A $5 \%$ corputational error is deemed acceptable because it is much smaller than the typical errors associated with uncertainties in specifying the release scenario. Tests indicate that the time step selection algorithm is generally successful for ensuring computational accuracy, but that it can fail if the user-entered time steps are longex than 50 to 60 times the air change times or the decay times of the nuclides that give significant contributions to the dose.

The exact details of how the time step selection is implemented can be seen by reviewing the computer codes. The discussion here is intended to give a general understanding of the conceptual basis for time step selection.

\subsection{CONHAB Time Steps.}

The method used to select a time step is most easily seen by first considering CONHAB. The equations used by CONHAB are valid only if the concentrations at the intakes do not change by too much during any time step. This means that TACT5 and FPFP_2 must write values to their respective.CNX files that have sufficient temporal resolution for use by CONHAB.

CONHAB computes the doses within a time step based on the integral of the control room concentrations during the time step. This is done using Simpson's rule on the concentrations at the start and end of the time step. The rules used to determine the time steps used by CONHAB are designed so that the value obtained by the Simpson's rule integration is a good approximation to the actual integral of the dose rate.

CONHAB first tries to take a time step that goes from one time that a control room or inflow parameter changes to the next time that a parameter changes. CONHAB then checks to see if this time step is sufficiently small to give good 
accuracy for the doses for each organ. If so, the time step is okay and is used. If not, CONHAB tries a smaller time step. This process is repeated until a satisfactory time step is found.

If the time step gets as small as 0.1 second and is still too large, a warning message is printed to the screen and to the. TAB file, the 0.1 second time step is taken, and computation proceeds. It is expected that this will not occur in actual use of the program. It is included as a safeguard against the program getting "stalled out" taking very small time steps.

A time step is considered to be small enough if at least one of the following conditions is met for every organ:

Rule 1. The dose rate for this organ changes by less than 5\% during this time step.

Rule 2. The contribution of this time step to the cumulative dose for this organ is less than 5\% of the contribution that was found for some time step that has already been tried.

Rule 3. If this dose rate were to continue until the end of the 30 day calculation then it would contribute less than 5\% to the cumulative dose already found for this organ.

These rules can be explained heuristically as follows:

Rule 1. This rule is illustrated in Figure 13.1. The rule states that a time step will be used if

where

$$
2 \text { ABS }[d(t)-d(t+d t)] /[d(t)+d(t+d t)]<0.05,
$$

$d(t)$ is the dose rate at time, $t$, and

$d(t+d t)$ is the dose rate at time $t+d t$.

Because control room concentrations behave as exponential functions, they are relatively smooth functions of time. Limiting the change in a time step to $5 \%$ means that the line segment used by simpson's rule will be very close to the function itself.

Rule 2. This rule is illustrated in Figure 13.2. Say that ConHaB has previously tried the time step between $t_{p}$ and $t_{p}+d t_{p}$. If that time step had been used it would have contributed $g_{n}$ anouht to the cumulative dose equal to the lightly shaded area in Figure 13.2. As can be seen from the figure, this area is not a very good estimate of the integral of the function. The time step currently being tried is represented by the darkly shaded area between times $t$ and $t+d t$. If the darkly shaded area is less than 0.05 of the lightly shaded area, then the trial time step is used. This rule says that the trial time step will contribute less than $5 \%$ of the total dose based on the using the darkly shaded area as a crude estimate for the total dose.

At the start of the release, control room concentrations are near 0 . Rule 2 is needed to "allow the computation to get going." Basically, this rule says that the time step is okay if its contribution to the dose is less than $5 \%$ of the contribution we have estimated for some larger time step. This condition allows the program to look ahead and conclude that this time integral will not contribute very much to the total dose. 
Rule 3. This rule is illustrated in Figure 13.3. The cumulative dose up to time $t$ is represented by the lightly shaded area. The darkly shaded area is the additional cumulative dose that would occur if the mean dose rate during the trial time step were to continue until 30 days. The trial time step is used if the darkly shaded area is less than 0.05 of the lightly shaded area. In mathematical form, this rule states that the trial time step will be used if

$$
0.5 \cdot[d(t)+d(t+d t)] \cdot[30 \text { days }-t]<0.05 \text { CumDose }(t) \text {, }
$$

where Cumbose ( $t)$ is the cumulative dose for time $t$. Note that if the trial time step is used, only the area between $t$ and $t+d t$ is added to the cumulative dose.

This rule is useful late in the computation. After a long time the concentrations may be very small compared to earlier in the release. This rule applies when the concentrations have become so small that they will contribute little additional dose between this time and the end of the computation.

If a trial time step is too large, a new smaller time step is tried. If the trial time step is acceptable, it may be possible for a larger time interval to be used for the next step. In either case the next time interval is chosen as the longest time interval that would be expected to meet either rule 1 or rule 2 for all organs at the $4 \%$ level. Using a time step expected to satisfy a $4 \%$ tolerance improves the efficiency of the program by reducing the $1 i k e l i-$ hood that the new time step will be too large.

\subsection{FPFP 2 Time Steps.}

The time steps used by FPFP_2 are based on similar methods to those in CONHAB. A time step is considered acceptable if at least one of the following conditions is met for all three of the groups (halogens, noble gasses, and particulates) in each node and for flow out of the end node:

Rule 1. The concentration changes by less than $5 \%$ during this time step.

Rule 2. The time integral of the concentration during this time step is less than $5 \%$ of the integral of the concentration for some other time step that has been tried.

Rule 3. The concentration is so small that the time integral of this concentration from this time to the end of the calculation or until 30 days (whichever is longer) is less than $5 \%$ of the time interval up to this time.

Rule 4. The length of this time interval is less than $5 \%$ of the time constant for this node. The time constant for a node is the e-folding time for the concentration of this form within this node (i.e., the time required for the concentration to make a $63 \%$ adjustment towards its steady state value). For rooms and ducts the time constant includes the effects of ventilation and removal processes. Filters and resistances do not have time constants associated with them. Any size time step is okay for a filter or a resistance.

The first three of these conditions are exactly parallel with the conditions 
in CONHAB. The fourth condition is added to take advantage of knowledge of the time constants of the nodes. A condition similar to the fourth condition could not be used in CONHAB because of the effects of radioactive decay on CONHAB concentrations.

\subsection{TACT5 Time Steps.}

TACT5 determines coefficients for the analytic expressions (exponential functions) that describe the concentrations of all of the nuclides in each of the nodes and the release rates to the environment. These are computationally exact (to within errors determined by the matrix solution methods) regardiess of the size of the time step used. The doses computed by TACT5 are obtained using the analytic expressions for the integrals of the environmental concentrations and are therefore also computationally exact. Thus, any size time step is adequate when TACT5 is run by itself.

However, when TACT5 is used within HABIT, the rates of nuclide release computed by TACT5 must have sufficient temporal resolution to insure that the doses computed by CONHAB are accurate. This is done by considering values for hypothetical dose rates and cumulative doses for a person exposed to air contaminated by nuclides released from the plant. This person is assumed to have a constant breathing rate and $X / Q$. Rules 1 through 3 are then applied to the dose rates and doses for each organ for this person. 


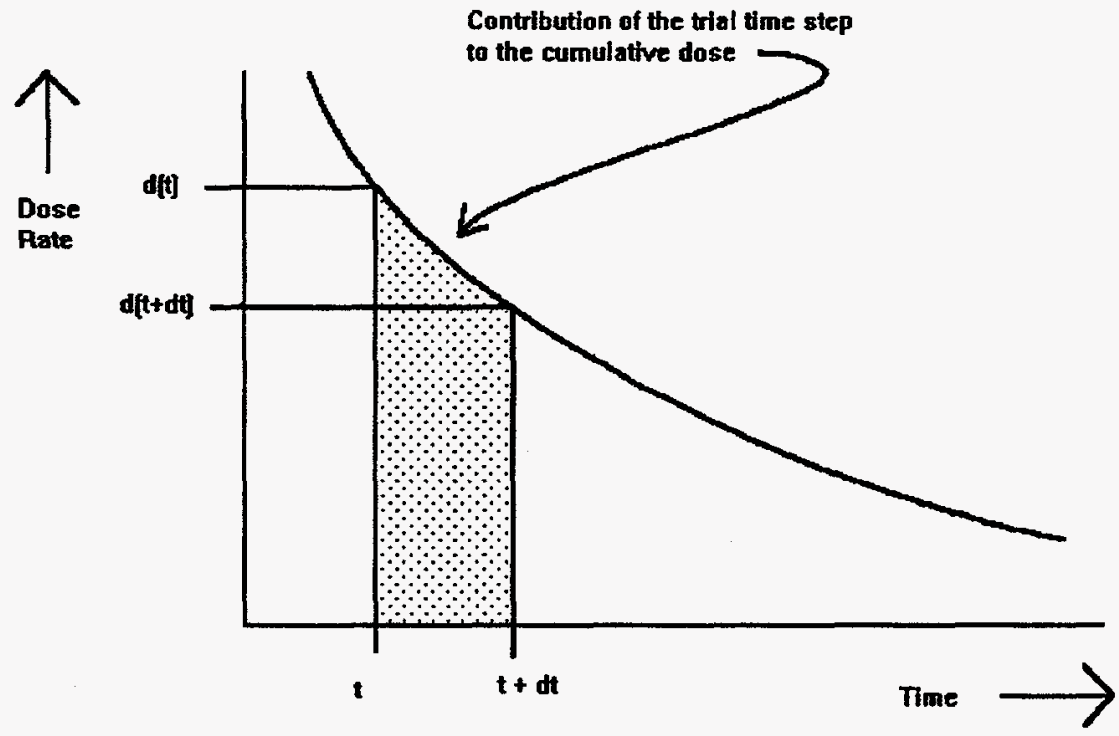

Figure 13.1: Schematic Diagram of Rule 1 for Determining Time Steps. 


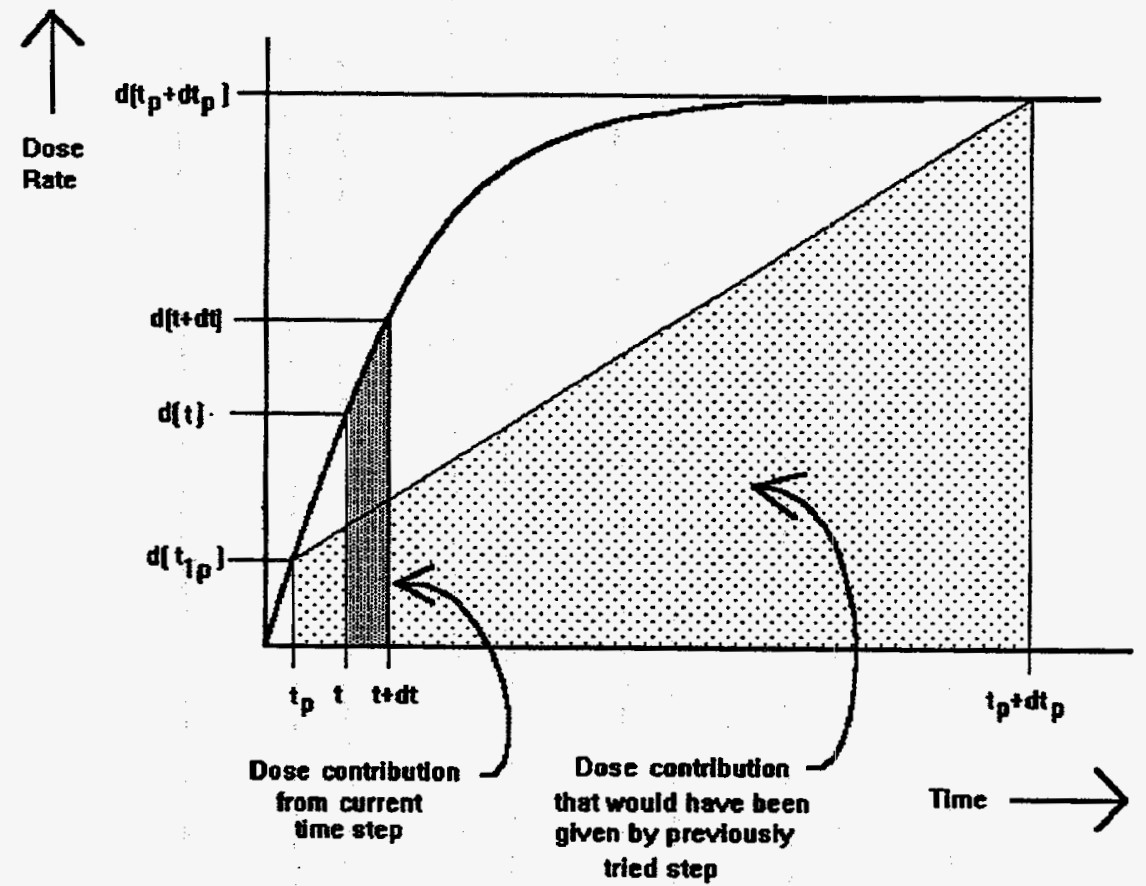

Figure 13.2: Schematic Diagram of Rule 2 for Determining Time Steps. 
HABIT Manual

NUREG/CR- 6210

Time steps

Rule 3

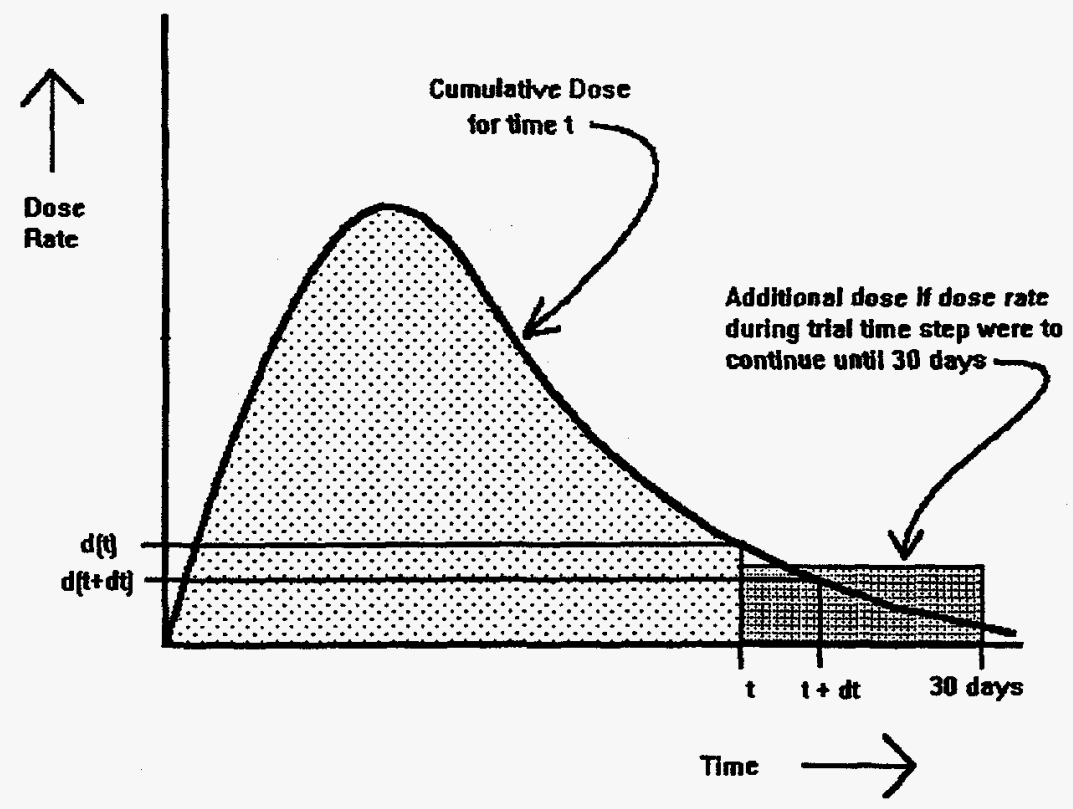

Figure 13.3: Schematic Diagram of Rule 3 for Determining Time steps. 

ANSI. 1978. American National Standard FORTRAN, X3.9-1978 (FORTRAN 77). American National Standards Institute, New York.

Ayer, J. E., A. T. Clark, P. Loysen, M. Y. Ballinger, J. Mishma, P. C. Owcarski, W: G. Gregory, and B. D. Nichols. 1988. Nuclear Fuel Cycle Facility Accident Analysis Handbook. NUREG-1320, U. S. Nuclear Regulatory Commission, Washington, D.C.

Bander, T. J. 1982. PAVAN: An Atmospheric Dispersion Program for Evaluating Design Basis Accidental Releases of Radioactive Materials from Nuclear Power stations. NUREG/CR-2858, PNL-4413, prepared by Pacific Northwest Laboratory for the U.S. Nuclear Regulatory Commission, Washington, D.C.

Bird, R. B., W. E. Stewart and E. N. Lightfoot. 1960. Transport Phenomena. John Wiley \& Sons, New York.

Briggs, G. A.. 1965. "A Plume Rise Model Compared with Observations." J. Air Pollution Control Assoc., $15(9): 433-438$.

Bolz, R. E., and G. L. Tuve. 1973. Handbook of Tables for Applied Engineering Science. CRC Press, Cleveland.

Chanin, D.I., J.I. Sprung, I.T. Ritchie, H-N Jow. 1990. MELCOR ACCident Consequence Code System (MACCS). Prepared by Sandia National Laboratories for the U.S. Nuclear Regulatory Commission. Washington, D.C., NUREG/CR-4691, SAND86-1652.

Denning, R. S., I. A. Gieseke, P. Cybulskis, K. W. Lee, H. Jordan, I. A. Curtis, R. F. Kelly, V. Kogan, P. M. Schumather. 1986. Radionuclide Release Calculations for Selected Severe Accident Scenarios, Vol I-V. NUREG/CR-4624, BMI-2139, prepared by Battelle Memorial Institute, Columbus, Ohio for the U.S. Nuclear Regulatory Commission, Washington, D.C.

Dickerson, M. H., and D. L. Ermak. 1988. "The Evaluation of Emergency Response Trace Gas and Dense Gas Dispersion Models." UCRI-99348, Lawrence Iivermore National Laboratory, Livermore, California. Presented at the American Meteorological Society Sponsored Advanced Studies Institute Short Course on the Meteorological Aspects of Bmergency Response, September 28-29, 1988 .

DiNunno, J.J., R.E. Baker, F.D. Anderson, and R.I Waterfield. 1962. "Calculation of Distance Factors for Power and Test Reactor Sites." U.S Atomic Energy Commission. TID-14844.

Driscoll, J. W.. 1988. Control Room Habitability Survey of Licensed Commercial Nuclear Power Generating Stations, NUREG/CR-4650, prepared by Argonne National Laboratory for the U.S. Nuclear Regulatory Commission, Washington, D.C. 
HABIT Manual

NUREG/CR- 6210

References

Eimutis, E. C., and M. G. Konicek. 1972. "Derivations of continuous functions for the lateral and vertical atmospheric dispersion coefficients." Atmos. Environ. $6: 859-863$.

Federal Guidance Report No. 11. 1988. Ifimiting Values of Radionuclide Intake And Air Concentration and Dose Conversion Factors For Inhalation, Submersion, and Ingestion. U.S. Environmental Protection Agency, EPA520/1-88-020.

Federal Guidance Report No. 12. 1993. External Exposure To Radionuclides In Air Water, and Soil. Environmental Protection Agency, EPA 420-R-93-081.

Gieseke, J. A., P. Cybulskis, R. S. Denning, M. R. Kuhlman, K. W. Lee, and H. Chen. 1984. Radionuclide Releases Under Specific LWR Accident Conditions, Vol. I-VI. BMI-2104, prepared by Battelle Memorial Institute, Columbus, Ohio for the U.S. Nuclear Regulatory Commission, Washington, D.C.

Gilpin, H. 1990. Control Room Habitability System Review Models. NUREG/CR5659, SAIC-90/1-54, prepared by Science Applications International Corporation for the U.S. Nuclear Regulatory Commission, Washington, D.C.

Hinds, W. T. 1969. "Peak-to-Mean Concentration Ratios from Ground Ievel Sources in Building Wakes." Atmos. Environ. 3:145-156.

Idso, S. B. 1972. "Systematic deviations of clear sky atmospheric thermal radiation from predictions of empirical formulae." 0 . J. Roy. Meteorol. Soc. $98: 399-401$.

International Commission on Radiological Protection. 1959. Report of Committee II on Permissible Dose for Internal Radiation. ICRP Publication 2, Peragmon Press, Oxford, England.

International Commission on Radiological Protection. 1979. Limits for Intakes of Radionuclides by Workers. ICRP Publication 30, Peragmon Press, Oxford, England.

Killough, G. G., C. I. Begovich, A. I. Sjoreen, and I. W. Bell. $1983 . \quad$ A Guide for the TACTIII Computer Code, NUREG/CR-3287, ORNL/TM-8763, prepared by Oak Ridge National Laboratory for the U. S. Nuclear Regulatory Commission, Washington, D.C.

Langhaar, J. W. 1953. "Cooling Pond May Answer Your Water Cooling Problem." Chem. Eng. $60(8): 194-198$.

Iederer, C.M., J.M. Hollander, and I. Perlman. 1967. Table of Isotopes. John Wiley and Sons, New York.

Mahlum, D. D., and L. B. Sasser. 1991. Evaluation of Exposure Limits to Toxic Gases for Nuclear Reactor Control Room Operators. NUREG/CR-5669, PNL-7522, prepared by Pacific Northwest Laboratory for the U. S. Nuclear Regulatory Commission, Washington, D.C.

Murphy, K. G., and K. M. Campe. 1974. "Nuclear Power Plant Control Room Ventilation System Design for Meeting General Design Criterion 19," Proceedings of the 13th AEC Air Cleaning Conference, August 12-15, 1974, San Francisco, California, CONF-740807, U.S. Atomic Energy Commission, Washington, DC. 
Oke, T. R. 1978. Boundary Layer Climates. Methuen \& Co., New York.

Owcarski. P. C., R. I. Schreck, and W. K. Winegardner. 1985. ICEDF: A Code for Aerosol Particle Capture in Ice Compartments. NUREG/CR-4130, PNL5379, prepared by Pacific Northwest Laboratory for the U. S. Nuclear Regulatory Commission, Washington, D.C.

Owcarski. P. C., K. W. Burk, J. V. Ramsdell and D. D. Yasuda. 1991. FPFP 2 : A Code for Following Airborne Fission Products in Generic Nuclear Plant Flow Paths. NUREG/CR-5658, PNL-7513, prepared by Pacific Northwest Laboratory for the U. S. Nuclear Regulatory Commission, Washington, D.C.

Paltridge, G. W. 1970. "Daytime long-wave radiation from the sky." $\Omega$. J. Roy. Meteorol. Soc. $96: 645-653$.

Paltridge, G. W., and C. M. R. Platt. 1976. Radiative Processes in Meteorology and Climatology. Elsevier Scientific Publishing Company, New York.

Powers, D. A. and S. B. Burson. 1993. A Simplified Model of Aerosol Removal by Containment Sprays. NUREG/CR-5966, SAND92-2689, prepared by Sandia National Laboratories for the U. S. Nuclear Regulatory Commission, Washington, D.C.

RamsdelI, J. V., and W. T. Hinds. 1971. "Concentration Fluctuations and Peak-to-Mean Concentration Ratios in Plumes from a Ground-Ievel Continuous Point Source." Atmos. Environ. 5:483-495.

Ramsdell, J. V. 1978. Impact of a Hanford Nuclear Eneray Center on Cloudiness and Insolation. PNL-6391. Pacific Northwest Laboratory, Richland, Washington.

Ramsdell, J. V., G. F. Athey and C. S. Glantz. 1983. MESOI Version 2.0: An Interactive Mesoscale Lagrangian Puff Dispersion Model With Deposition and Decay. NUREG/CR-3344, PNL-4753 prepared by Pacific Northwest Laboratory for the U. S. Nuclear Regulatory Commission, Washington, D.C.

Ramsdell, J. V. 1988. Atmospheric Diffusion for Control Room Habitability Assessments. NUREG/CR-5055, PNL-6391, prepared by Pacific Northwest Laboratory for the U. S. Nuclear Regulatory Commission, Washington, D.C.

Ramsdell, J. V. 1990a. "Alternatives to Current Procedures Used to Estimate Concentrations in Building Wakes." PNL-SA-17941. Presented at the 21st DOE/NRC Nuclear Air Cleaning Conference, August 13-16, 1990, San Diego, California.

Ramsdell, J. V. 1990b. "Diffusion in Building Wakes for Ground-Level Releases." Atmos. Environ. 24B(3):377-388

Ramsdel1, J. V. 1991. EXTRAN: A Computer Code for Estimating Concentrations of Toxic Substances at Control Room Air Intakes. NUREG/CR-5656, PNL7510 , prepared by Pacific Northwest Laboratory for the $U$. $S$. Nuclear Regulatory Commission, Washington, D.C. 
Ramsdell, J. V., and C. J. Fosmire. 1995. Atmospheric Dispersion Estimates in the Vicinity of Buildings. PNL-10286, prepared by Pacific Northwest Laboratory for the U. S. Nuclear Regulatory Commission, Washington, D.C.

Reid, R. C., J. M. Prausnitz and B. E. Poling. 1987. The Properties of Gases \& Liquids. McGraw-Hill Book Company, New York. $741 \mathrm{p}$.

Rosenberg, N. J., B. L. Blad and S. B. Verma. 1983. Microclimate: The Biological Environment. John Wiley \& Sons, New York.

Sagendorf, J. F., J. T. Goll and W. F. Sandusky. 1982 . X00Do0: Computer program for the Meteorological Evaluation of Routine Effluent Releases at Nuclear Power Stations. PNL-4380 prepared by Pacific Northwest Laboratory for the U. S. Nuclear Regulatory Commission, Washington, D.C.

Soffer, L., S.B. Burson, C.M. Ferrell, R.Y. Lee, and J.N. Ridgely. 1994. Accident Source Terms for Iight-Water Nuclear Power Plants. NUREG-1465.

Swinbank, W. C. 1963. "Long-wave radiation from clear skies." ㅇ․ J. Roy. Meteorol. Soc. $89: 339-348$.

Unrein, P. J., C. A. Pelletier, J. E. Cline, and P. G. Voilleque. 1985. "Transmission of Radioiodine Through Sampling Lines." In Proceedings of the 18 th DOE Nuclear Airborne Waste Management and Air Cleaning Conference, CONF-840806, M. W. First, ed., pp. 116-125. U.S. Department of Energy, Washington, D.C.

West, D.B. and H.E. Gilpin. 1988. User's Guide for the TACT5 Computer Code. Prepared by Science Applications International Corporation for the U.S. Nuclear Regulatory Commission, Washington, D.C., NUREG/CR5106, SAIC-88/3023.

Widner, T. W., G. W. Graham, K. E. Shank, I. A. Burchfield, and P. G. Voilleque. 1985. "Determination of Radionuclide Transmission Through Effluent Monitor Sample Lines for Susquehanna Steam Electric Station." Presented at the 1985 . Health Physics Society Meeting.

Wing, J. 1979. Toxic Vapor Concentrations in the Control Room Following a Postulated Accidental Release. NUREG-0570. U. S. Nuclear Regulatory Commission, Washington, D.C. 
Offsite

12 DOE Office of Scientific and Technical Information

14 Accident Evaluations Branch Division of Systems Technology Office of Nuclear Regulatory Research

U. S. Nuclear Regulatory Commission Washington, D.C. 20555

C. E. Ader

J. H. Schaperow (12)

C. G. Tinkler

7 Division of Technical support Office of Nuclear Reactor Regulation 1 U. S. Nuclear Regulatory Commission Washington, D.C. 20555

R. I. Emch

A. M. Gill

J. J. Hayes

J. Y. Lee

S. A. Boynton

D. R. Carter

J. L. Minns

1 Division of Engineering Office of Nuclear Reactor Regulation U. S. Nuclear Regulatory Commission Washington, D.C. 20555

R. I. Parczewski

1

Division of Systems Safety and

1 Analyis office of Nuclear Reactor Regulation U. S. Nuclear Regulatory Commission Washington, D.C. 20555

S. R. Jones

Director

Division of systems Technology

Office of Nuclear Regulatory Research

U. S. Nuclear Regulatory Commission

Washington, D.C. 20555

ATTN : Barbara Stehlin,

Management Analyst

2

Science Applications International Corp.

1865 Terminal Dr. Suite 202

Richland, WA

J. Misima

P. Owczarski

Brookhaven National Laboratory

Building 130

P.O. Box 5000

Upton. Long Island, NY 11973-5000

Partha Neogy

2 Sandia National Laboratory

P.O. Box 5800

Albuquerque, NM 87185

S. Humphries

D. Monroe

\section{Onsite}

34

Pacific Northwest Iaboratory

K. J. Allwine

K9-30

J. G. Droppo

C. J. Fosmire

$\mathrm{K} 6-55$

J. V. Ramsdell

K9-30

L. B. Sasser

S. A. Stage (20)

G. J. Vargo

Records Center (2)

Publishing Coordination

Technical Library (5)
$\mathrm{K} 9-30$

P7 -53

K9-30

K3-56

$\mathrm{k} 3-70$

$\mathrm{K} 1-06$

P8-55 


\begin{tabular}{|c|c|}
\hline $\begin{array}{l}\text { U.S. NUCLEAR REGULATORY COMMISSION } \\
\text { BIBLIOGRAPHIC DATA SHEET } \\
\text { (See instructions on the reverse) }\end{array}$ & \multirow[t]{2}{*}{$\begin{array}{l}\text { 1. REPORT NUMBER } \\
\text { (Assigned by NRC. Add Vol, Supp., Rev., } \\
\text { and Addendum Numbers, it any.) } \\
\text { NUREG / CR- } 6210 \\
\text { PNL-10496 }\end{array}$} \\
\hline 2. TITLE AND SUBTITLE & \\
\hline \multirow{3}{*}{$\begin{array}{l}\text { Computer Codes for Evaluation of Control Room Habitability } \\
\text { (HABIT) }\end{array}$} & DATE REPORT PUBLISHED \\
\hline & \begin{tabular}{|l|l|} 
MONTH & YEAR \\
\end{tabular} \\
\hline & \\
\hline \multirow[t]{2}{*}{$\begin{array}{l}\text { 5. AUTHOR(S) } \\
\text { S. A. Stage }\end{array}$} & 6. TYPE OF REPORT \\
\hline & $\begin{array}{l}\text { 7. PERIOD COVERED (1nclusive Dates) } \\
\text { September } 1992 \text { to } \\
\text { February } 1995\end{array}$ \\
\hline \multicolumn{2}{|l|}{$\begin{array}{l}\text { 8. PER FORMING ORGANIZATION - NAME AND ADDRESS (If NRC, provide Division. Office or Region, U.S. Nuclear Re, } \\
\text { name and mailing address.) } \\
\text { Pacific Nort thwest Laboratory } \\
\text { Richland, WA } 99352\end{array}$} \\
\hline \multicolumn{2}{|c|}{$\begin{array}{l}\text { 9. SPONSORING ORGANIZATION - NAME AND ADDRESS IIf NRC, tvpe "Same as above", if contractor, provide NRC Division, Office or Region. U.S. Nuclear Regulatory Commission, } \\
\text { and mailing zddress./ } \\
\text { Division of Systems Technology } \\
\text { Office of Nuclear Regulatory Research } \\
\text { U.S. Nuclear Regulatory Commission } \\
\text { Washington, DC 20555-0001 }\end{array}$} \\
\hline \multicolumn{2}{|l|}{$\begin{array}{l}\text { 10. SUPPLEMENTARY NOTES } \\
\text { J. Schaperow, NRC Project Manager }\end{array}$} \\
\hline \multicolumn{2}{|c|}{$\begin{array}{l}\text { This report describes the computer codes for evaluation of control room habitability (HABIT). HABIT is a } \\
\text { package of computer codes designed to be used for the evaluation of control room habitability in the event of } \\
\text { an accidental release of toxic chemicals or radioactive materials. Given information about the design of a } \\
\text { nuclear power plant, a scenario for the release of toxic chemicals or radionuclides, and information about the } \\
\text { air flows and protection systems of the control room; HABIT can be used to estimate the chemical exposure } \\
\text { or radiological dose of control room personnel. HABIT is an integrated package of several programs that } \\
\text { previously needed to be run separately and required considerable user intervention. These are EXTRAN, } \\
\text { CHEM, TACT5, FPFP } 2 \text {, and CONHAB. New input routines have been written for these routines using } \\
\text { data input windows. These are designed for easy use entering, reviewing and revising the data. The } \\
\text { programs can now be run in sequence as an integrated package. Improvements have been made in the } \\
\text { computational methods used by some of the routines. The programs produce files containing ASCII tables of } \\
\text { values and output files that can readily be imported into a commercial spreadsheet to be graphed or for } \\
\text { further computations. }\end{array}$} \\
\hline \multirow{5}{*}{$\begin{array}{l}\text { 12. KEY WORDS/DESCR!PTORS (List words or phrases that will assist researchers in locating the report.) } \\
\text { Computer code, HABIT, radioactive material, radionuclides, } \\
\text { control room }\end{array}$} & 13. AVAILABILITY STATEMENT \\
\hline & \begin{tabular}{|l} 
Unlimited \\
14. SECURITYCLASSIFICATION
\end{tabular} \\
\hline & $\begin{array}{l}\text { (This Pagei } \\
\text { Unclassified } \\
\text { (This Report) }\end{array}$ \\
\hline & \begin{tabular}{|l} 
Unclassified \\
15. NUMBER OF PAGES
\end{tabular} \\
\hline & 16. PRICE \\
\hline
\end{tabular}

